\title{
Hennepin County's Experience with Heavy-Duty Ethanol Vehicles
}

\author{
Trucking Research Institute \\ Alexandria, Virginia \\ and \\ Hennepin County Central Mobile Equipment Division \\ Hopkins, Minnesota
}

NREL Technical Monitor: Mike Frailey

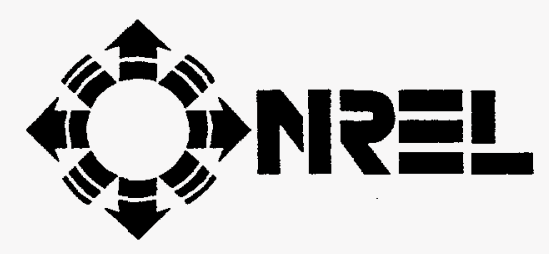

Alternative Fuels Hotline: 1-800-423-1DOE

Alternative Fuels Data Center World Wide Web Site: http://www.afdc.doe.gov

National Renewable Energy Laboratory

1617 Cole Boulevard

Golden, Colorado 80401-3393

A national laboratory of the

U.S. Department of Energy

Managed by the Midwest Research Institute

For the U.S. Department of Energy

Under Contract NO. DE-AC36-83CH10093

MASTER

Prepared under Subcontract Number ZAZ-3-12223-01-107183

January 1998

DISTRIBUTION OF THIS DOCUMENT IS UNLMTTES 
This publication was reproduced from the best available camera-ready copy subminted by the subcontractor and received no editorial review at NREL.

\section{NOTICE}

This report was prepared as an account of work sponsored by an agency of the United States government. Neither the United States government nor any agency thereof, nor any of their employees, makes any warranty, express or implied, or assumes any legal liability or responsibility for the accuracy, completeness, or usefulness of any information, apparatus, product, or process disclosed, or represents that its use would not infringe privately owned rights. Reference herein to any specific commercial product, process, or service by trade name, trademark, manufacturer, or otherwise does not necessarily constitute or imply its endorsement, recommendation, or favoring by the United States govemment or any agency thereof. The views and opinions of authors expressed herein do not necessarily state or reflect those of the United States government or any agency thereof.

Available to DOE and DOE contractors from:

Office of Scientific and Technical Information (OSTI)

P.O. Box 62

Oak Ridge, TN 37831

Prices available by calling (423) $576-8401$

Available to the public from:

National Technical Information Service (NTIS)

U.S. Department of Commerce

5285 Port Royal Road

Springfield, VA 22161

(703) $487-4650$ 


\section{DISCLAIMER}

Portions of this document may be illegible electronic image products. Images are produced from the best available original document. 


\section{Executive Summary}

From November 1993 to October 1996, Hennepin County, which includes Minneapolis, Minnesota, field-tested two heavy-duty snowplow/road maintenance trucks fueled by ethanol. The overall objective of this program was to collect data from original equipment manufacturer (OEM) alternative fuel heavy-duty trucks, along with comparable data from a similarly configured diesel-powered vehicle, to establish economic, emissions, performance, and durability data for the alternative fuel technology. The U.S. Department of Energy (DOE) provided funding for the project through the National Renewable Energy Laboratory (NREL) and the American Trucking Association (ATA) Foundation's Trucking Research Institute (TRI). This is the second of three ethanol demonstrations that NREL has funded. The first demonstration took place in Decatur, nlinois, at Archer Daniels Midland (ADM) Trucking, Incorporated. Applicable data from the ADM report also appear in this report.

The two trucks, International Paystar models F5070, were equipped with a U.S. emissionscertified Detroit Diesel Corporation (DDC) model 6V-92TA ethanol engine. The engine is rated at 300 horsepower and is fueled by E95, a fuel composed of $95 \%$ anhydrous ethanol and $5 \%$ light hydrocarbon denaturant. These ethanol trucks, along with an identical third truck equipped with a U.S. emissions-certified DDC 6V-92TA diesel engine, were operated year round to maintain the Hennepin county roads. In winter, the trucks were run in 8-hour shifts plowing and hauling snow from urban and suburban roads. For the rest of the year, the three trucks were used to repair and maintain these same roads.

As a result of this project, a considerable amount of data was collected on E95 fuel use, as well as maintenance, repair, emissions, and operational characteristics. Maintenance and repair costs of the E95 trucks were considerably higher primarily due to fuel filter and fuel pump issues.

From an emissions standpoint, the E95 trucks emitted less particulate matter and fewer oxides of nitrogen but more carbon monoxide and hydrocarbons. Overall, the E95 trucks operated as well as the diesel, as long as the fuel filters were changed frequently.

This project was a success in that E95, a domestically produced fuel from a renewable energy source, was used in a heavy-duty truck application and performed the same rigorous tasks as the diesel counterparts. The drawbacks to E95 as a heavy-duty fuel take the form of higher operational costs, higher fuel costs, shorter range, and the lack of over-the-road infrastructure. 


\section{Contents}

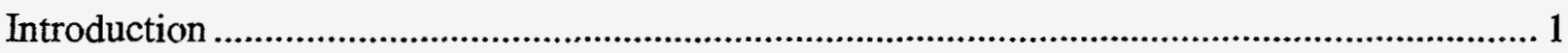

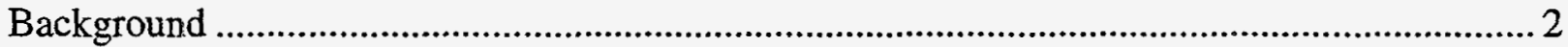

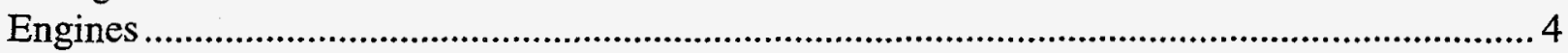

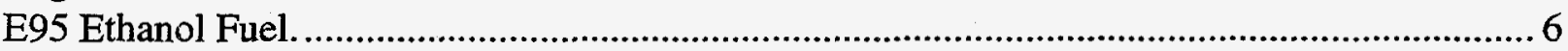

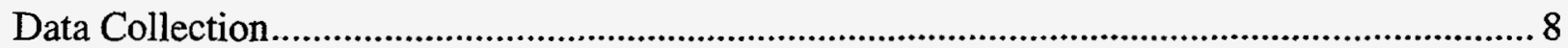

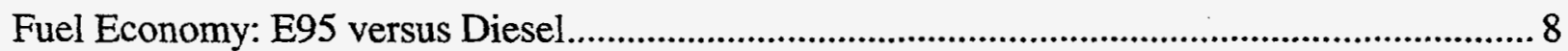

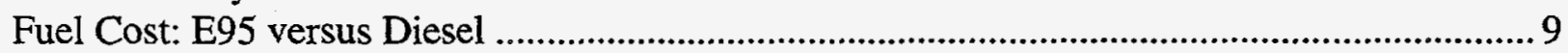

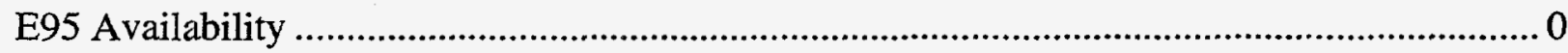

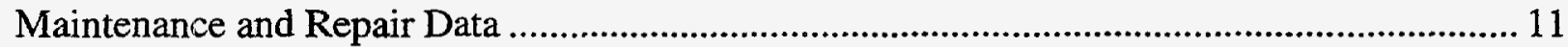

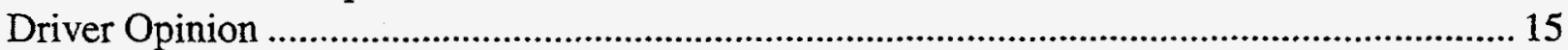

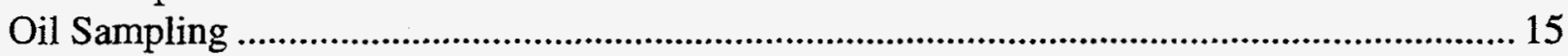

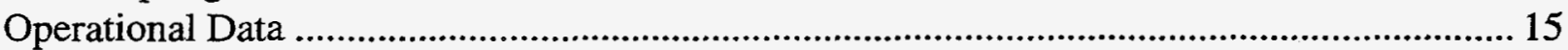

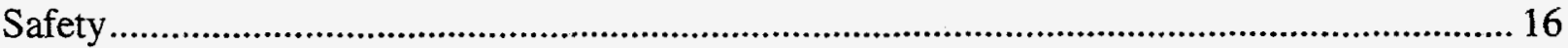

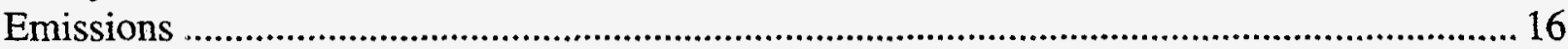

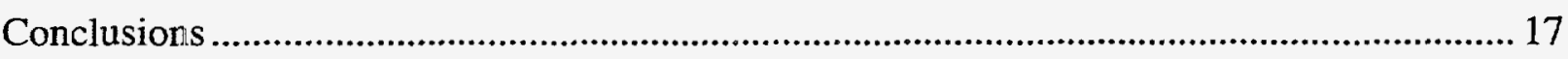

Appendix 1. Collection Form to Gather Data: Driver Option............................................1-1

Appendix 2. Map of Demonstration Area .................................................................. 2-1

Appendix 3. Specifications for E95 Ethanol Fuel .......................................................... 3-1

Appendix 4. Maintenance Costs by Truck System and Year ............................................. 4-1

Appendix 5. Repair History by Truck System ............................................................ $5-1$

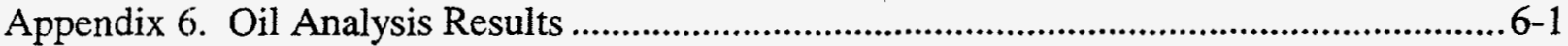

Appendix 7. Summary of Emissions Testing ............................................................... 7-1

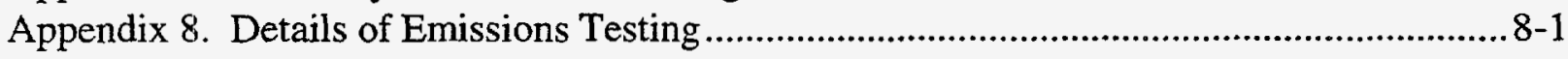

\section{List of Tables}

Table 1. Comparison of Properties of Potential Diesel Fuel Components............................... 7

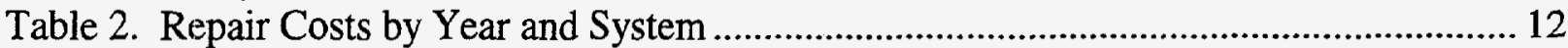

Table 3. Total Maintenance Cost by System ....................................................................... 12

\section{List of Figures}

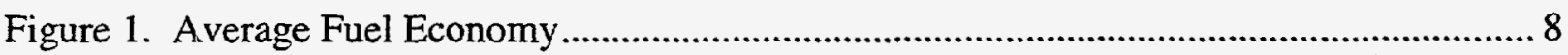

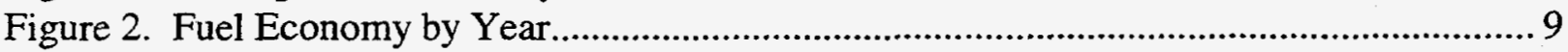

Figure 3. Total Maintenance Cost by Truck................................................................ 10

Figure 4. Total Maintenance Cost per Mile ............................................................... 12

Figure 5. Total Maintenance Cost by System.................................................................. 13 


\section{Introduction}

The American transportation sector uses more than $30 \%$ of all the energy consumed annually in this country. Conventional hydrocarbon fuels, primarily gasoline and diesel fuel, represent the current sources of energy for transportation. Naturally occurring hydrocarbon reserves, however, exist in finite quantities and are very limited in geographic extent. Proven worldwide hydrocarbon reserves are being depleted faster than they are being discovered, and more than $50 \%$ of the petroleum used for energy in America each year is imported.

Heavy-duty trucks and urban transit buses account for a significant portion of the U.S. transportation sector. For the most part, these vehicles are powered by compression-ignition diesel fuel engines, which typically emit high levels of oxides of nitrogen $\left(\mathrm{NO}_{\mathrm{x}}\right)$ and black particulate smoke during operation. The black smoke is not only unsightly, but has proven to be carcinogenic.

Ethanol, also known as ethyl alcohol, is a renewable energy resource generated by biologically fermenting simple glucose sugars. At this time, the ethanol industry in the United States has an annual production capacity of approximately 1.6 billion gallons. Although ethanol can be made from a wide variety of feedstocks, corn is generally used to produce ethanol in this country because of its abundance and relatively low price considering the amount of ethanol that it yields.

Ethanol has been successfully used as an additive in gasoline for many years. This fuel formulation, also called "gasohol," is composed of $90 \%$ unleaded gasoline and $10 \%$ ethanol. In this application, ethanol can be considered a petroleum extender, an octane enhancer, and an oxygenate additive to gasoline.

From an emissions standpoint, numerous studies have shown that gasoline blended with $10 \%$ ethanol reduces carbon monoxide (CO) emissions from internal combustion engines by as much as $25 \%$. Ethanol-blended gasolines are being used in many of the nation's cities currently in nonattainment for $\mathrm{CO}$ levels. Until recently, very little was known, however, about emissions from vehicles designed to operate on higher percentage blends of ethanol fuel.

Until the 1990s, the use of high-percentage blends of ethanol fuels for transportation was very limited. Henry Ford was the first American automobile manufacturer to see the potential of ethanol as a fuel in the early 1900s. Many of his early Model A automobiles were capable of operating on ethanol fuel rather than gasoline. Since then, only a few isolated testing programs (most performed by vehicle and engine manufacturers and containing proprietary information) have been designed to test, demonstrate, and evaluate the use of ethanol as a viable alternative transportation fuel in light-, medium-, and heavy-duty vehicles.

The overall objective of this project was to demonstrate and collect data on the use of a highpercentage ethanol blend of fuel in a heavy-duty truck application. This project also represents the first public demonstration of the use of ethanol fuels as a viable alternative to conventional diesel fuel in a heavy-duty snowplow/road maintenance application. 


\section{Background}

It is an impressive sight to see a 60,000-pound snowplow rolling back a layer of snow in a perfect arc to the roadside. Snowplowing is an extremely demanding assignment that can test the limit of a heavy-duty truck design. Snowplow/maintenance trucks work as part bulldozer and part dump truck, often in extreme cold, loaded to their capacity, cutting through ice and snow. Hennepin County in Minneapolis, Minnesota, offers a perfect cold weather environment for testing alternative fuel heavy-duty trucks.

Hennepin County is an urban county of a little more than 1 million people; it makes up about half the Minneapolis/St. Paul metropolitan area. The city of Minneapolis is the county seat. The county has largely typical midwestern topography with a mix of flat farmland, lakeshore, and moderate rolling hills. Much of the county road system comprises multi-lane city streets with curb and gutter, medians, center islands, multiple turn lanes, and traffic control devices. A small portion of the system remains essentially rural with two-way roads, unpaved shoulders, and little in the way of traffic control other than an occasional stop sign.

In 1993, the county began field-testing two ethanol-powered trucks with an identically specified diesel control truck. The county vehicle numbers were 3221,3228 , and 3220 , respectively. For the remainder of this report, we will refer to them as ethanol \#1, ethanol \#2, and diesel control, respectively. The demonstration ran for 3 years from November 1993 through October 1996. The trucks were used by the Department of Public Works Division of Road and Bridge Maintenance Operations. To ensure uniformity and consistency in reporting data from drivers, we asked for volunteer drivers who were willing to stay with the trucks for the full 3 years of the study. We were fortunate that long-term, experienced, and proficient drivers volunteered for the project. Collectively, the three drivers have 62 years of experience driving heavy-duty trucks. They provided weekly data on the performance of the trucks and on the fuel usage. The drivers completed a standard form provided by the ATA, shown in Appendix 1.

All the heavy-duty trucks owned by Hennepin County are used to maintain the county's highway system. This system includes about 600 miles of roads with about 1600 lane-miles.

The Class 8 dump trucks are equipped in the winter with a $12-\mathrm{ft}$ reversible front plow, a $10-\mathrm{ft}$ underbody scraper blade, and a tailgate spreader.

The trucks are International Paystar 5000, model F5070, with the following specifications:

GVWR

Wheelbase

Cab-to-Axle

Front Axle

Rear Axle

Engine
49,500

188 in.

120 in.

18,000 \& set back

$38,0004.78$ ratio

6V-92TA DDEC

300 h.p. @ 2,100 rpm

$50 \mathrm{ft} .1 \mathrm{~b}$. torque @1,300 rpm
Transmission: Fuller RTX11708LL

8 speed w/double low

14.56:1 max. reduction

Body: 15-ft Aluminum

Telescopic Hoist

Engine Power Take Off (PTO) 
This configuration is standard for county dump trucks, and this truck is the workhorse of the county fleet. The three Navistar trucks were purchased with non-U.S. emissions-certified DDC 6V-92TA diesel engines. Navistar, the chassis OEM, did not offer the ethanol or diesel versions of the DDC 6V-92TA with U.S. emissions certification as a commercial option. Consequently all three trucks were repowered with U.S.-emissions-certified 6V-92TA engines by the local DDC dealer; two with the ethanol version and one with the diesel.

All the heavy-duty trucks owned by Hennepin County are purchased primarily for the snowplowing function. The complete snow and ice control operation is essential to maintaining a transportation network in Minnesota. The typical winter duty cycle consists of snowplowing, snow removal, and ice control. For this reason, the county road system is divided into segments so that one truck can plow the full segment in one 8-hour shift. One truck and driver are assigned to each of these segments or routes. The routes for the three trucks in this demonstration project were:

- Diesel control-The most rural route in the project. At least half the route consisted of two-lane roads with no curb and gutter. The remainder of the route was a mix of two-lane roads without curb and gutter, and two-lane or four-lane suburban streets with curb and gutter.

- Ethanol \#1-All urban streets, four lanes with both right and left turn lanes, traffic signals, and curb and gutter.

- Ethanol \#2-Mostly urban streets, four lanes with turn lanes, traffic signal, curb and gutter. Some sections are two lanes with no curb and gutter.

Appendix 2 contains a map of Hennepin County and these routes.

In a standard duty cycle, the drivers are called to start plowing at 2:45 a.m. The driver loads his truck and begins to plow, clearing the main road lanes first and then spending the rest of the 8-hour shift plowing the shoulders or clearing the roadway full width to the curbs and any turn lanes. The beauty of the dump truck, of course, is that the driver can spread salt/sand mix or salt to control ice while he is plowing. Subsequent shifts will either repeat this cycle, or move into more intensive clean-up plowing, either on an early start or during regular work hours.

Other typical winter work cycles include hauling materials from one location to another. Salt, for example, is frequently moved from the county's main supply, at the department's headquarters, to the outlying truck stations so those facilities have adequate quantities for their snow removal activities. When there is no snow, the drivers may begin by patrolling their assigned routes looking for icy spots and salting them. When the plowing and salting is done, the drivers will often assist with Public Works projects, which generally entail hauling aggregates, street sweepings, dirt, trees and brush, broken and removed concrete, construction refuse, gravel fill, and snow. 
Later in the winter, when the snow has narrowed the streets, the trucks help in joint city/county operations to make the streets more passable. A city identifies the street most in need of widening, and provides a grader, an operator, and other laborers. The grader cuts out the snow/ice that has accumulated at the road edge and forms it into a windrow. The county provides a large loader-mounted snow blower and several tandem-axle dump trucks. The blower moves down the windrow and blows the snow into the dump bodies of the trucks. One truck follows another, pulling under the blower shoot as soon as the lead truck is loaded and out of the way. The combined operation efficiently widens the streets. The trucks then haul and dump the snow in a city-designated storage area.

The winter weather during the 3-year project is described below.

1993-94 Total snowfall: 55.7 inches

Average temperature: 12.4 degrees $\mathrm{F}$

Temperature range: -26 to 34 degrees $F$

1994-95 Total snowfall: 29.6 inches

Average temperature: 22.6 degrees $\mathrm{F}$

Temperature range: -5 to 38 degrees $F$

1995-96 Total snowfall: 55.5 inches

Average temperature: 16.7 degrees $F$

Temperature range: -16 to 36 degrees $F$

In the summer, these trucks become essential tools in the county's paving and pavement maintenance programs. As a rule, the county contracts most major road work except for bituminous overlay and seal coat programs, which are completed by county employees and equipment. In this operation trucks make continuous rounds to haul millings (created during resurfacing process) to a disposal site, deliver hot asphalt to the paver, or take seal coat chips to the seal coating operation.

The summer and winter work cycles described above were consistent throughout the demonstration and were performed by the diesel control and ethanol trucks alike.

\section{Engines}

The DDC Model 6V-92TA (with the TA denoting turbocharged and aftercooled) powerplant was selected as the engine for the two ethanol trucks. The 6V-92TA alcohol fuel engine is a veeconfiguration, six cylinder, two-cycle motor capable of producing up to 300 horsepower. The methanol version of the engine was first developed in 1986 and emissions certified (by the U.S. Environmental Protection Agency [EPA] and the California Air Resources Board [CARB]) in 1991 for use in urban transit buses in California. DDC's previous experiences with the methanol 6V-92TA engine aided the development and subsequent emissions certification of the ethanol -6V-0-92TA in 1992. DDC considered both alcohol engines and standard production engines. 
DDC selected the 6V-92TA engine to develop as an alcohol fuel engine for at least two reasons: (1) the 6V-92TA diesel engine was used in about $80 \%$ of the urban transit buses currently on the road, and (2) the two-cycle engine was easier to convert to alcohol fuel than a four-cycle engine. A two-cycle engine removes combustion products close to the bottom of the piston stroke by means of a blower that pushes out the exhaust gases (called "scavenging"). Scavenging causes mixing of hot exhaust gases with the new fuel mixture to be combusted. The presence of these hot exhaust gases in the cylinder raises the fuel temperature, making compression ignition of low-cetane-number fuels (such as ethanol) possible. As a result, two-cycle engines have a distinct advantage over four-cycle engines in compression igniting fuel with high auto-ignition temperatures.

DDC also modified some of the components on the ethanol 6V-92TA engines. The first of these major modifications was to the Detroit Diesel Electronic Controls (referred to as DDEC II), which contains the electronic control module (ECM) and the electronic unit (fuel) injectors (EUI). The ECM is the on-board computer for the engine that controls various engine operations under continuously varying conditions to optimize performance, fuel economy, and emissions. The ECM receives electronic signals from the truck's driver in addition to engine-mounted sensors. The electronic hardware in the ECM contains a PROM (Programmable Read Only Memory) encoded with the specific engine performance characteristics (such as horsepower rating, torque curve, and maximum engine speed. To use ethanol fuel, the ECM must be specifically programmed for ethanol fuel at the factory or by a DDC field engineer.

The second part of the DDEC II unit, the EUI, contains an electronically controlled solenoid valve that meters and times fuel input to the cylinders. Because ethanol contains only about $60 \%$ of the energy of diesel fuel per unit volume, more ethanol fuel is required to generate the same amount of power in the engine. This is accomplished by using two larger (relative to diesel) ethanol-resistant fuel pumps and by increasing the diameter of the holes in the injector tip. The EUI can then inject the proper amount of ethanol fuel into the cylinder at the right time without reducing the engine performance.

Next, the bypass air system was modified. This system provides the correct metering and mixing of retained hot exhaust gases and fresh air in order to achieve the proper ethanol compressionignition temperature. The bypass air system is controlled by the DDEC II unit and changes system settings based on changes in engine operating conditions.

The ethanol 6V-92TA engines also use a glow plug system that has been modified for use with the DDEC II system. The glow plugs are electronically heated and are used to help start the engine. The glow plugs remain on (heated) for 1 minute prior to starting the engine and remain on until the engine coolant reaches normal operating temperature.

Another major modification to the ethanol engine is an increased compression ratio compared to the conventional diesel version. The ethanol engine has a compression ratio of 23:1 compared to 18:1 for the diesel. This increase is needed to ensure complete combustion of the ethanol fuel in the cylinder and to maximize engine performance and torque. 
Finally, the fuel systems of the two ethanol trucks were modified. Because ethanol is more corrosive to certain metal, plastic, and rubber parts, stainless-steel fuel tanks and fuel lines were added.

\section{E95 Ethanol Fuel}

Ethanol (also called ethyl alcohol) is an oxygenated hydrocarbon with the chemical formula of $\mathrm{C}_{2} \mathrm{H}_{5} \mathrm{OH}$. Ethanol is a by-product of the fermentation of simple glucose sugar by yeasts. Anhydrous ethanol contains 76,000 Btu of energy per gallon. Energy contents and other properties of ethanol are compared to other fuels in Table 1.

The "proof" of an alcohol is defined as twice the percentage of alcohol in the solution. For example, a mixture of $90 \%$ pure ethanol and $10 \%$ water is referred to as "180-proof" ethanol.

For all practical purposes, there are two types of industrial-grade ethanol: anhydrous and hydrated. Anhydrous ethanol is defined as at least $99.5 \%$ pure ethanol, with less than $0.5 \%$ water by volume. Anhydrous ethanol is also called " 200 proof" ethanol or gasoline grade ethanol, as this is the concentration of ethanol blended with gasoline to make "gasohol." Hydrated ethanol is any other ethanol blend containing more than $0.5 \%$ water by volume. In either case, fuel-grade ethanol musk be denatured with $2 \%$ to $5 \%$ denaturant to make it unfit for human consumption. Otherwise, it is highly taxed by the U.S. Department of Treasury, Bureau of Alcohol, Tobacco, and Firearms (ATF) as beverage-grade alcohol. Some of the denaturants commonly used include methanol, benzene, toluene, natural gasoline, kerosene, and unleaded gasoline.

The cetane number, also found in Table 1, is a measure of the ease (or difficulty) by which a fuel can be compression ignited. Fuels with high cetane numbers have low autoignition temperatures and short ignition delay times. Ethanol has a low cetane number, less than 5 , compared to 40 to 55 for diesel fuel. This indicates that the alcohol fuels have higher autoignition temperatures relative to diesel fuel. This is reflected in the autoignition temperatures of $\sim 600$ degrees $\mathrm{F}$ for diesel fuel and 793 degrees $\mathrm{F}$ for ethanol. Hence, the requirement of glow plugs to autoignite the E95 fuel in the 6V-92TA engines.

Diesel fuel is a complex mixture of hydrocarbons, and the composition can vary from "batch to batch." Because of this variation, the energy content of \#2 diesel fuel is assumed to average about 127,000 Btu per gallon (Btu/gal).

The fuel formulation used in the two 6V-92TA truck engines in this project is a blend of $95 \%$ anhydrous ethanol and 5\% denaturant. This fuel is called $\mathrm{E} 95$, but it can also be called $\mathrm{E}(\mathrm{d})-100$, with the "d" standing for denatured. For consistency, the term E95 will be used throughout this report whenever $95 \%$ anhydrous ethanol and 5\% denaturant fuel is discussed.

DDC supplied an E95 fuel specification for use with its 6V-92TA engines (shown in Appendix 3), which includes an optional lubrication additive called "Lubrizol". One problem encountered with using ethanol fuels is that they do not have the inherent lubricating properties 


\begin{tabular}{|c|c|c|c|c|c|c|c|c|c|c|c|}
\hline Property & $\begin{array}{l}\text { DF-2 } \\
\text { Diesel }\end{array}$ & $\begin{array}{l}\text { Fischer- } \\
\text { Tropsch }\end{array}$ & $\begin{array}{l}\text { Bio- } \\
\text { diesel }\end{array}$ & Gasoline & $\mathrm{CNG}$ & $\begin{array}{c}\text { Propane } \\
\text { HD-5 }\end{array}$ & $\begin{array}{c}\text { Methano } \\
1\end{array}$ & Ethanol & Methylal & $\begin{array}{c}\text { Dimethyl } \\
\text { ether }\end{array}$ & $\begin{array}{c}\text { Diethyl } \\
\text { ether }\end{array}$ \\
\hline Formula & 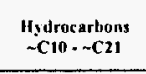 & $\begin{array}{c}\text { Principally } \\
\mathrm{C}_{\mathrm{n}} \mathrm{H}_{2 n+2} \\
\end{array}$ & $\begin{array}{c}\begin{array}{c}\text { Various sits sunt } \\
\text { csters }\end{array} \\
\end{array}$ & $\begin{array}{c}\text { Hydrocarthons } \\
\text { c-c-c9 }\end{array}$ & $\begin{array}{c}\text { Principallys } \\
\mathrm{CH}_{4}\end{array}$ & $\begin{array}{c}\text { Principally } \\
\text { C.3Hs }\end{array}$ & Снзон & $\mathrm{C}_{2} \mathrm{H}_{5} \mathrm{OH}$ & $\begin{array}{c}\mathrm{CH}_{3} \mathrm{OCH}_{2} \mathrm{OCH} \\
\end{array}$ & $\mathrm{CH}_{3} \mathrm{OCH}$ & $\mathrm{C}_{2} \mathrm{H}_{3} \mathrm{OC}_{2} \mathrm{H}_{5}$ \\
\hline Boiling Point, ${ }^{\circ} \mathrm{F}$ & $\begin{array}{c}370- \\
650\end{array}$ & $\begin{array}{c}350- \\
670\end{array}$ & $\begin{array}{c}360- \\
640\end{array}$ & $80-437$ & n.a. & n.a. & 149 & 172 & 107 & -13 & 94 \\
\hline Reid Vapor Pressure, psi @100 $\mathrm{F}$ & $<0.2$ & n.a & n.a & $8-15$ & n.a. & 170 & 4.6 & 2.3 & $12.2^{\mathrm{c}}$ & 116 & 16.0 \\
\hline Cetane Number & $40-55$ & $>74$ & $>48$ & $13-17$ & low & low & low & $<5^{b}$ & 49 & $>55$ & $>125^{\mathrm{a}}$ \\
\hline Autoignition Temperature, ${ }^{\circ} \mathrm{F}$ & $\sim 600$ & $\sim 600$ & - & 495 & 990 & 870 & 867 & 793 & 459 & 662 & 320 \\
\hline Stoichiometric Air/Fuel Ratio, Wt./Wt. & 15.0 & 15.2 & 13.8 & 14.5 & 16.4 & 15.7 & 6.45 & 9.0 & 7.1 & 8.9 & 11.1 \\
\hline Flammability Limits, Vol. \%: $\quad$ Rich & 7.6 & - & - & 6.0 & 13.9 & 9.5 & 36.9 & 19.0 & $14.9^{\mathrm{c}}$ & $27.0^{\mathrm{d}}$ & $\begin{array}{c}9.5 \\
36.0^{\mathrm{d}}\end{array}$ \\
\hline Flammability Limits, Vol. \%: & 1.4 & - & - & 1.0 & 5.0 & 2.4 & 7.3 & 4.3 & $3.3^{\mathrm{c}}$ & $3.4^{\mathrm{d}}$ & $1.9^{\mathrm{d}}$ \\
\hline Lower Heating Value, Btu/lb & 18,200 & 18,600 & 16,500 & 18,500 & 20,750 & 19,940 & 8,570 & 11,500 & 10,200 & 12,100 & 14,600 \\
\hline Lower Heating Value, Btu/gal & 128,700 & 121,300 & 120,910 & 115,400 & & 83,900 & 56,800 & 76,000 & 73,000 & 66,600 & 86,500 \\
\hline Lower Heating Value, Btu/SCF & & & & & 1,033 & & & & & & \\
\hline Viscosity, centipoise at (temp) ${ }^{\circ} \mathrm{F}$ & $40(68)$ & $2.1(100)$ & $3.5(100)$ & $3.4(68)$ & - & - & $0.59(68)$ & $1.19(68)$ & - & - & $0.23(68)$ \\
\hline Specific Gravity@60 $60^{\circ} \mathrm{F}$ & 0.860 & 0.783 & 0.880 & 0.750 & - & 0.506 & 0.796 & 0.794 & 0.86 & 0.66 & 0.714 \\
\hline Density, Ib/gal & 7.079 & 6.520 & 7.328 & 6.246 & - & 4.21 & 6.629 & 6.612 & 7.16 & 5.50 & 5.946 \\
\hline Note (See below) & $\mathrm{e}$ & $\mathrm{h}$ & $\mathrm{b}$ & $\mathrm{e}$ & $f$ & $\mathrm{~h}$ & $\mathrm{e}$ & $\mathrm{e}$ & c & $\mathrm{b}$ & $\mathrm{b}$ \\
\hline \multicolumn{12}{|c|}{$\begin{array}{l}\text { * Table compiled by N.R. Sefer, Southwest Research Institute. } \\
\text { a. Inferred from ignition delay } \\
\text { b. Recent measurement at Southwest Research Institute } \\
\text { c. Naegeli, D. W. ,and Weatherford, W. D. Jr. "Practical Ignition Limits for Low Molecular Weight Alcohols," Fuel 68, 45 (1989) } \\
\text { d. NFPA 325 M, Fire Hazard Properties of Flammable Liquids, Gases, Volatile Solids (1977), Copyright 1977 National Fire Protection Association, Inc., Batterymarch Park, } \\
\quad \text { Quincy, MA 02269 } \\
\text { e. Table on gasoline and gasohol from Alcohols and Ethers, API Publication 4261, second edition, (July 1988). } \\
\text { f. Liss, W.E., et al., "Variability of Natural Gas Composition in the US", GRI 92/0123, (March 1992). } \\
\text { g. Alternative Fuels Special Report, Diesel Progress, Engines and Drives, (December 1993). } \\
\text { h. Composition information from ASTM D } 1635 \text { Standard Specification for LPG. Calculations after Perry's Chemical Engineers'Handbook, 6th ed., (1984). }\end{array}$} \\
\hline
\end{tabular}


of petroleum-based fuels. To ensure upper cylinder lubrication, an additive developed in California for the methanol buses, Lubrizol, is added to fuel in very low concentrations. The recommended amount of Lubrizol is $0.06 \%$, by volume, in the fuel mixture. Hennepin County was able to contract an E95 fuel supplier and confirm the DDC recommended fuel specifications, including the specified amount of Lubrizol.

\section{Data Collection}

The overall objective of this program was to collect data from OEM alternative fuel heavy-duty trucks, along with comparable data from a similarly configured diesel-powered vehicle to establish emissions, performance, and durability data for alternative fuel technology. The data were collected from drivers and mechanics on paper forms provided by TRI, and then forwarded to NREL. TRI compiled the data and entered the information into NREL's Alternative Fuels Data Center (AFDC) via modem.

\section{Fuel Economy: E95 versus Diesel}

Assuming \#2 diesel fuel contains about $127,000 \mathrm{Btu} / \mathrm{gal}$ and E95 fuel contains $78,000 \mathrm{Btu} / \mathrm{gal}$, the ethanol trucks should travel about $60 \%$ of the distance the diesel trucks travel on a gallon of fuel, if the trucks have the same thermal efficiency. On a volume basis, the average fuel economy over the project was 4.6, 2.6, and 2.2 miles per gallon (mpg) for the diesel control, ethanol \#1, and ethanol \#2, respectively (shown in Figure 1). The ethanol trucks traveled about $48 \%$ to $57 \%$ of the distance traveled by the diesel trucks. This indicates that the thermal efficiency of the ethanol trucks was somewhat less than that of the diesel truck. Although plowing snow represents only a fraction of the miles driven in a month, it is not unusual to see the overall fuel economy of a truck drop by one or two miles per gallon in the winter months. Both ethanol trucks dropped to $1 \mathrm{mpg}$ at some time during the project.

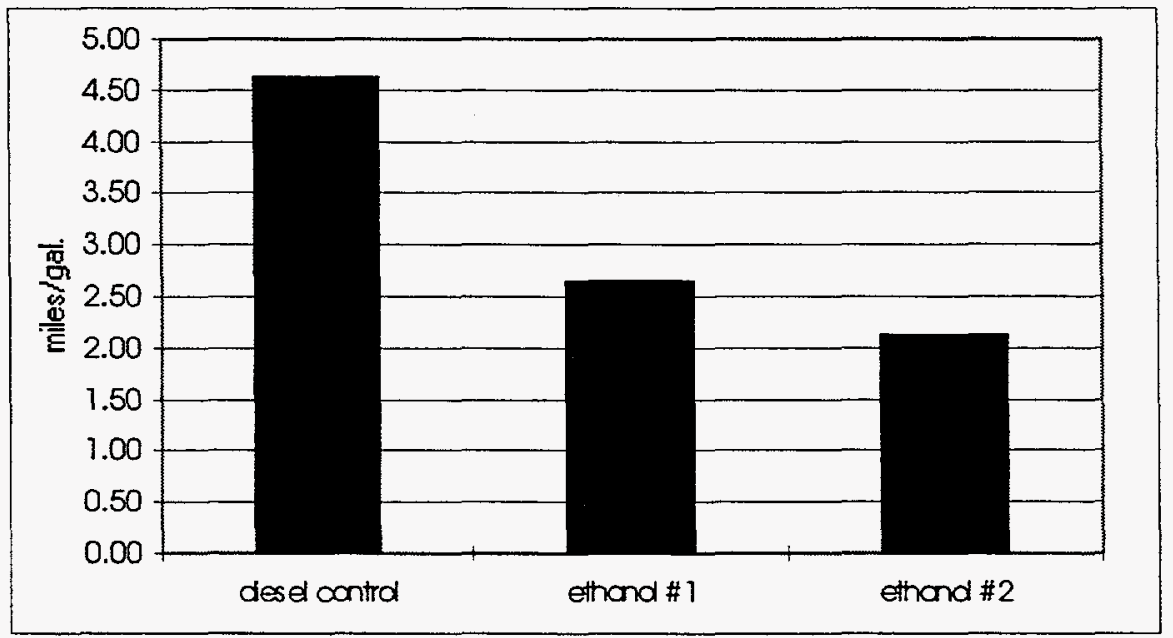

Figure 1. Average Fuel Economy 


\section{Fuel Cost: E95 versus Diesel}

The cost of ethanol fuel is high, about twice as much as diesel. In the beginning of the project, diesel cost the county $\$ 0.80 /$ gallon and ethanol $\$ 1.40 /$ gallon. At the project end, diesel cost $\$ 0.90 /$ gallon and ethanol cost $\$ 1.80 /$ gallon. The county cost is a delivered price for bulk purchases and includes a state fuel tax of $\$ 0.20 /$ gallon. The county does not pay federal fuel tax. The most comprehensive picture of fuel costs is illustrated in the comparison of fuel cost per mile by year (shown in Figure 2). This graph indicates that, from 1994 on, the E95 fuel cost was three to four times more (per mile) than the diesel fuel cost.

Application of the alcohol tax credit can greatly reduce the cost of ethanol and the operation costs of a fleet of ethanol vehicles. Congress developed the alcohol fuel credit in response to the energy crisis of the late 1970s and early 1980s. The intent was to foster growth of the alcohol industry by subsidizing non-petroleum-based alcohol used as fuel so the cost for the end user would be comparable to traditional hydrocarbon fuels. The tax credit is accomplished in two ways: (1) by granting a $\$ 0.054$ per gallon partial excise tax exemption for $10 \%$ alcohol blended fuels (gasohol) and (2) by allowing a $\$ 0.54$ per gallon income tax credit for ethanol used as a fuel. The income tax credit is available to taxpayers who: (1) "produce" or blend an alcohol mixture, or (2) sell or use for business $100 \%$ straight alcohol placed in the vehicle's fuel supply tank by the taxpayer. Because E95 fuel is composed of 95\% ethanol and 5\% denaturant, $95 \%$ of the $\$ 0.54$ (or $\$ 0.513$ per gallon E95) per gallon tax credit can be taken. However, because Hennepin County is a state agency and exempt from federal taxes, it cannot file for the alcohol tax credit.

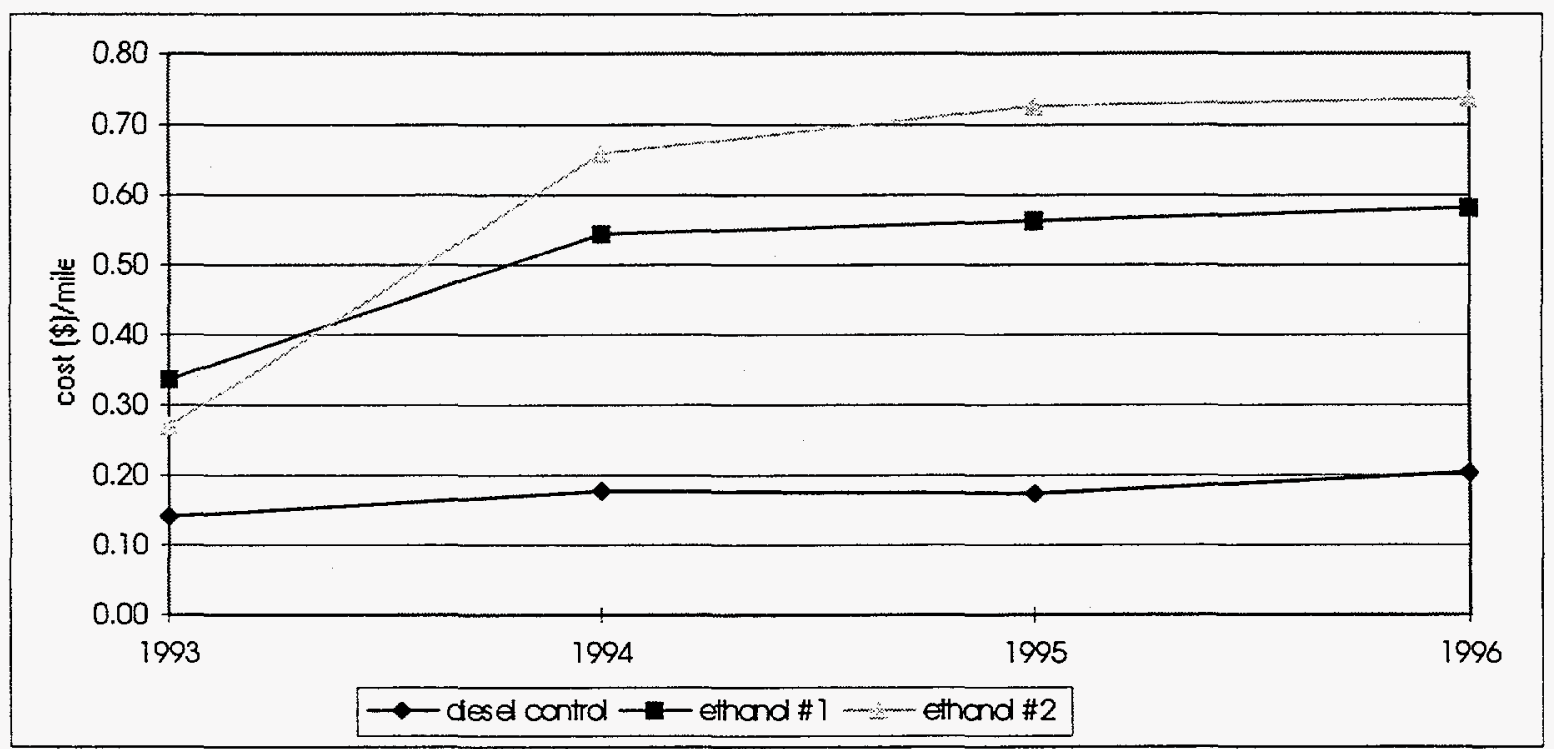

Figure 2. Fuel Cost per Mile by Year 


\section{E95 Availability}

Minneapolis-St. Paul is a major metropolitan center in the agricultural Midwest. The area has a history of supporting ethanol production and its use as an automotive fuel. There has been a long-standing promotion of gasohol, a $10 \%$ blend of ethanol and unleaded gasoline. The county has used gasohol in its automotive fleet since 1983. In addition, the cities are also a moderate nonattainment area for carbon monoxide and the Clean Air Act requires that this area use an oxygenated fuel during winter months. For this application, ethanol has been the oxygenate of choice. These factors have combined to produce plentiful supplies of ethanol in the metropolitan area.

The county bid this fuel the same as all other bulk fuel purchases and never experienced any delays in delivery. The supplier was able to provide E95 that met the manufacturer's specification, as shown in Appendix 3, with the recommended additive "Lubrizol" (part no. 23509970). The Lubrizol is added to the fuel as a detergent, to eliminate lube oil contamination of injectors, and to increase fuel lubricity. The county is convinced it did not have any maintenance problems that resulted from poor fuel quality.

\section{Maintenance and Repair Data}

The total maintenance and repair cost for the diesel control truck was $\$ 21,549$ as opposed to $\$ 26,101$ and $\$ 22,512$ for ethanol \#1 and ethanol \#2, respectively (shown in Figure 3). The higher costs for the ethanol trucks were evident in each year throughout the project except in 1996 (shown in Table 2). The higher cost for the diesel control in 1996 was primarily due to component replacement costs, which is directly related to the difference in accumulated mileage.

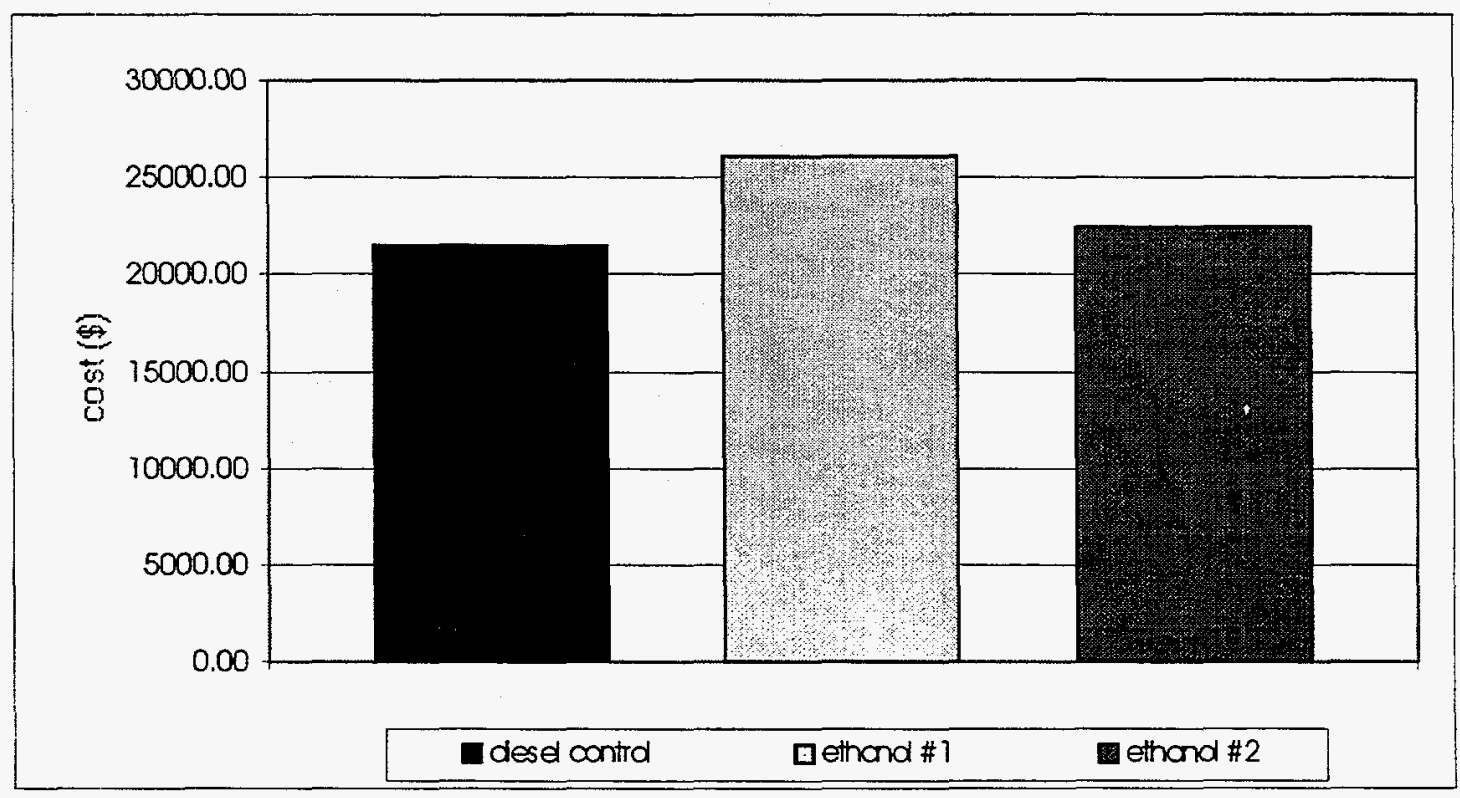

Figure 3. Total Maintenance Cost by Truck 
The usage of the three trucks did vary more than intended. The mileage on the three trucks at end of the project was 81,861 for the diesel control, 61,002 for ethanol \#1, and 32,526 for ethanol \#2. The reason for the unexpected variance was that we had, for consistency, assigned specific drivers to each vehicle for the duration of the test. The lesser availability of one of the drivers during the project caused a difference in truck usage. To factor in the difference in accumulated mileage, the total maintenance cost per mile was charted for the three trucks. Over the life of the project, the costs per mile were $\$ 0.26, \$ 0.43$, and $\$ 0.69$ for the diesel control, ethanol \#1, and ethanol \#2, respectively (shown in Figure 4).

Table 2. Repair Costs by Year and System

\begin{tabular}{|c|c|c|c|c|c|c|}
\hline & \multicolumn{3}{|c|}{1993 Repair Costs } & \multicolumn{3}{|c|}{1994 Repair Costs } \\
\hline & Diesel control & ethanol \#1 & ethanol \#2 & diesel control & ethanol \#1 & ethanol \#2 \\
\hline Cab/Body & 0.00 & 21.50 & 215.02 & 47.95 & $1,431.63$ & 448.42 \\
\hline PM'S & 180.51 & 0.00 & 0.00 & 379.26 & 401.91 & 329.32 \\
\hline Chassis GRP & 553.23 & 918.30 & 75.25 & 198.66 & 645.41 & 556.18 \\
\hline Drive Train & 0.00 & 0.00 & 0.00 & 258.00 & 64.50 & 96.75 \\
\hline Electrical & 0.00 & 0.00 & 0.00 & $1,406.97$ & 803.65 & $1,352.35$ \\
\hline Power Plant & 62.09 & 807.94 & 402.08 & 488.00 & $2,157.12$ & $1,905.16$ \\
\hline Accessories & 187.22 & 260.66 & 238.00 & $1,865.73$ & $2,802.66$ & $2,630.36$ \\
\hline Special Appl. & 78.81 & 238.14 & 143.31 & 0.00 & 129.00 & 0.00 \\
\hline Hydraulics & 107.50 & 0.00 & 21.50 & 190.36 & 374.11 & 237.95 \\
\hline Misc. Work & $4,256.39$ & $4,312.27$ & $4,526.91$ & $1,061.75$ & 778.20 & $1,055.20$ \\
\hline Misc.-New Prep & 377.17 & 0.00 & 0.00 & & & \\
\hline Total-Prep & $1,546.53$ & $2,246.54$ & $1,095.16$ & & & \\
\hline \multirow[t]{3}{*}{ Total } & $5,425.75$ & $6,558.81$ & $5,622.07$ & $5,896.68$ & $9,588.19$ & $8,611.69$ \\
\hline & \multicolumn{3}{|c|}{1995 Repair Costs } & \multicolumn{3}{|c|}{1996 Repair Costs } \\
\hline & Diesel control & ethanol \#1 & ethanol \#2 & diesel control & ethanol \#1 & ethanol \#2 \\
\hline Cab/Body & 630.26 & 383.74 & 270.95 & $1,008.75$ & 671.06 & 577.01 \\
\hline PM'S & 401.21 & 570.29 & 519.43 & 187.22 & 232.50 & 239.65 \\
\hline Chassis GRP & $1,425.38$ & 299.11 & 451.47 & $2,102.76$ & $2,165.10$ & 183.88 \\
\hline Drive Train & 68.55 & 64.50 & 86.00 & 21.50 & 296.64 & 21.50 \\
\hline Electrical & 745.04 & $1,491.89$ & $1,289.03$ & $1,293.96$ & 799.17 & 912.92 \\
\hline Power Plant & 983.16 & $1,615.15$ & 384.22 & $1,411.54$ & $1,197.77$ & $1,775.22$ \\
\hline Accessories & 534.36 & 781.25 & 630.67 & $1,058.95$ & 680.97 & 601.00 \\
\hline Special Appl. & 0.00 & 179.34 & 53.75 & 32.25 & 64.50 & 251.18 \\
\hline Hydraulics & 343.15 & 103.24 & 334.71 & 654.25 & 560.87 & 643.37 \\
\hline Misc. Work & 704.99 & $1,079.91$ & $2,325.26$ & 498.90 & $1,028.81$ & $1,253.76$ \\
\hline Total & $5,836.10$ & $6,568.42$ & $6,345.49$ & $8,270.08$ & $7,697.39$ & $6,459.49$ \\
\hline
\end{tabular}




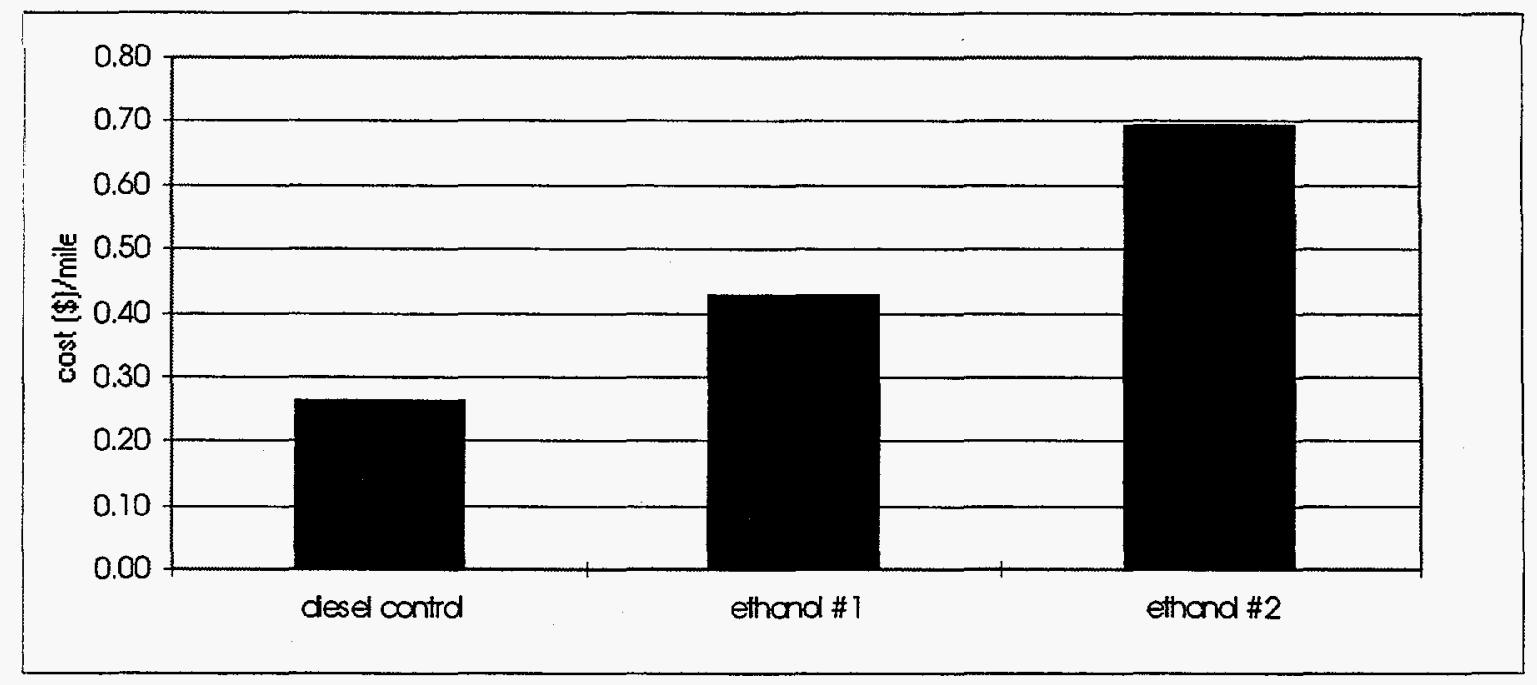

Figure 4. Total Maintenance Cost per Mile

To determine whether these costs resulted from the alternative fuel, the repair costs were broken down by truck systems. Table 3 shows the total maintenance cost by truck system. The detailed components that make up these systems can be seen in Appendix 4, which details repair costs by system and year. The most expensive systems for the 3 vehicles were chassis, for the diesel control, and power plant for both ethanol \#1 and ethanol \#2. Power plant maintenance and repair ranked number 4 for the diesel control. This can also be seen graphically in Figure 5.

Table 3. Total Maintenance Cost by Systems

\begin{tabular}{|c|c|c|c|}
\hline & diesel control & ethanol \#1 & Ethanol \#2 \\
\hline Cab /Body & $1,686.96$ & $2,507.93$ & $1,511.40$ \\
\hline PM'S & $1,148.20$ & $1,204.70$ & 948.36 \\
\hline Chassis GRP & $4,280.03$ & $4,027.92$ & $1,406.82$ \\
\hline Drive Train & 348.05 & 425.64 & 204.25 \\
\hline Electrical & $3,445.97$ & $3,094.71$ & $3,554.30$ \\
\hline Power Plant & $2,944.79$ & $5,777.98$ & $4,466.68$ \\
\hline Accessories & $3,646.26$ & $4,525.54$ & $4,100.03$ \\
\hline Special Appl. & 111.06 & 610.98 & 448.24 \\
\hline Hydraulics & $1,295.26$ & $1,038.22$ & $1,237.53$ \\
\hline $\begin{array}{c}\text { Misc.-New } \\
\text { Prep }\end{array}$ & $2,642.81$ & $2,886.92$ & $4,634.22$ \\
\hline Total & $\mathbf{2 1 , 5 4 9 . 3 9}$ & $\mathbf{2 6 , 1 0 0 . 5 4}$ & $\mathbf{2 2 , 5 1 1 . 8 3}$ \\
\hline
\end{tabular}

The difference in maintenance and repair costs between the diesel control and the ethanol trucks is mainly attributable to the E95 fuel. Most of the components that are unique to the ethanol trucks are in the power plant system. The few remaining ethanol-specific components are categorized in cab/body or electrical and are insignificant relative to the non-ethanol component costs that make up those systems. 
Most of the power plant repairs on the ethanol trucks were fuel system repairs. Both the primary and secondary fuel filters had to be replaced frequently. The fuel filters for the ethanol engines are much less porous than the diesel fuel filters to stop fine dirt from damaging the injectors and cylinders. The DDC 6V-92TA ethanol engine requires a 10- micron primary and a 1-micron secondary filter, where the DDC 6V-92TA diesel engine only requires a 40-micron primary and 5-micron secondary filter. Because of the smaller porosity, ethanol filters tend to clog more frequently from dirt and debris that enter the fuel system in the normal course of operation. The finer filters are required because ethanol does not have the lubricating properties inherent to petroleum-based fuels. Consequently, engines that burn ethanol are far less tolerant of impurities. We replaced the filters once each year on the diesel truck compared to eight or nine times for the ethanol trucks. Although the primary ethanol filter price came down $\$ 72$ during the project, the ethanol filters, primary and secondary, were still $\$ 107$ more expensive than the diesel filters. In the end, the primary and secondary ethanol filters cost $\$ 73$ and $\$ 50$, respectively. The diesel primary and secondary cost $\$ 8$ each. The total costs for fuel filter replacements were $\$ 48$, $\$ 1,843$, and $\$ 1,667$, for the diesel control, ethanol \#1, and ethanol \#2, respectively.

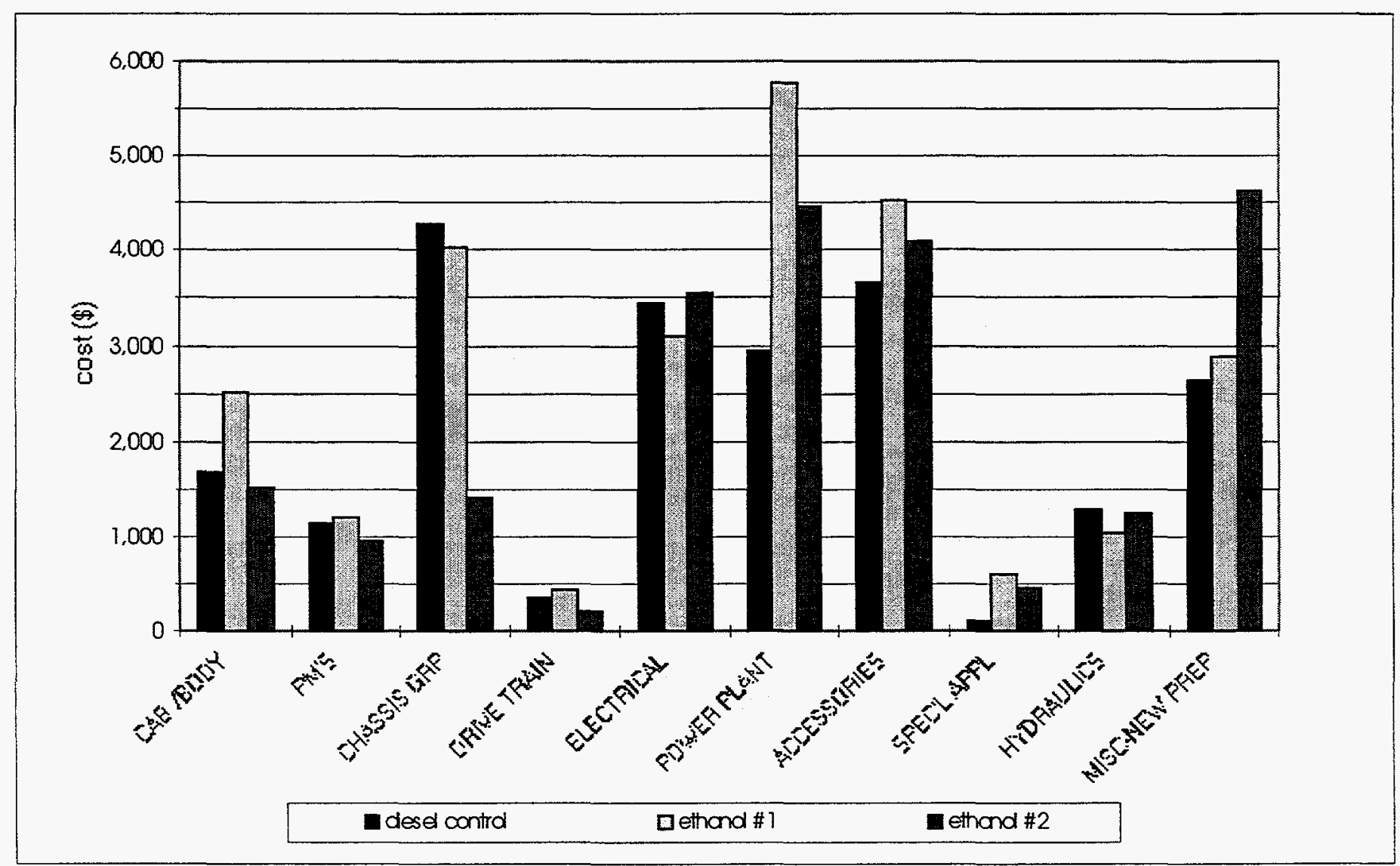

Figure 5. Total Maintenance Cost by Systems 
The ethanol trucks also experienced fuel pump and fuel sending unit problems. Ethanol fuel pumps differ from diesel fuel pumps. They need to deliver almost twice as much fuel as a diesel fuel pump as a result of the lower heating value of ethanol. They also require special alcoholresistant components. Both ethanol trucks required fuel pump replacements during the project. The fuel pump was replaced under warranty on ethanol \#1 and the county replaced the fuel pump on ethanol $¥ 2$. The total bill for this one repair was $\$ 1,229$, making it one of the more expensive repairs. Both trucks also had recurring problems with the fuel sending units and other miscellaneous fuel system repairs. In total, fuel system repair costs for the ethanol trucks were $\$ 2,782$ and $\$ 2,623$, compared to $\$ 205$ for the diesel truck. These costs account for most of the difference in repair costs for the three trucks.

Both ethanol trucks also had recurring problems with the electronic engine controls, but the problem was particularly troublesome on ethanol \#2. The self-diagnostic codes (instrument panel warning system) repeatedly displayed false alarms for the cooling system and crankcase oil pressure. The county referred these problems to the dealer because they developed while the trucks were under warranty. The initial response from the dealer was that these were indeed false codes and the ECM was reset. When the false codes reoccurred, the dealer thought the problem was caused by bad sensors or a programming glitch with the ECM. Neither of these possibilities developed into a permanent fix and eventually the drivers came to just ignore the warnings.

In 1996, the last year of the project, both ethanol trucks started to require glow plug replacement. Glow plug replacement at this mileage is premature relative to diesel engines. Ethanol \# 1 had three glow plugs replaced at a total cost of $\$ 324.56$ and ethanol \#2 had four glow plugs replaced at a total cost of $\$ 332.97$

Although it is not being attributed to E95, it should be noted that ethanol \#1 had a catastrophic engine failure at the end of the project. A cylinder head gasket failure allowed antifreeze into the cylinder. According to the engine dealer, the failure of the head gasket could have been caused by a gasket material failure, incorrect cylinder liner height, or incorrect head bolt torque. Although none of these failures can be discounted, the most likely cause is a failure or flaw in the gasket material. We do not believe the failure was fuel related.

Also worth noting, the new vehicle preparation costs are not included in these repair figures. These costs- $\$ 3,879, \$ 4,312$, and, $\$ 4,527$ for the diesel control, ethanol \#1, and ethanol \#2, respectively - are part of the cost of placing a truck in service and are not related to the alternative fuel. Mounting snow control equipment is quite labor intensive and staff time accounts for most of these costs. Because the preparation costs overstate the cost of repairs in the "miscellaneous" category, they were not included in the repair cost analysis.

The complete repair history for all trucks, arranged chronologically and by truck systems, is given in Appendix 5. 


\section{Driver Opinion}

The fuel delivery system problems were the most troublesome to the drivers. As the fuel filters began to clog, drivers noted hard starting, stalling, hesitation, and cutting in and out. The more clogged the filters became, the more frequently the problems occurred. Sometimes the trucks would stall in traffic, creating a tense situation for the driver.

Fuel range was also a problem for the drivers. The drivers frequently expressed concern about having enough fuel to complete their routes. Although neither of the E95 trucks ever ran out of fuel, the drivers retained a level of nervousness about the short range, especially during the winter months.

The electronic problems were mildly annoying. The recurring false codes weakened the credibility of the diagnostic instrumentation and frequent trips to the engine dealer disrupted the normal work cycle. In the end, the drivers ignored the error messages.

Finally, the drivers reported a lack of power in the ethanol trucks. During the most strenuous assignments, such as hauling sand or aggregate on a grade, the drivers contended that the ethanol trucks had less power than the diesel. However, it should be noted that the county did not quantitatively substantiate these opinions.

Overall, when asked, the drivers stated they would not pick the ethanol trucks if given a choice of which truck to drive.

\section{Oil Sampling}

The county has been using oil analysis on its heavy equipment for about 20 years, and has had good luck in extending the oil change interval and in detecting engine problems early. The oil analysis for the ethanol trucks went smoothly with no abnormalities in all instances but one. The last oil sample on ethanol \#1 revealed serious problems. However, by the time we received the results, the engine had already failed. As indicated previously, a head gasket failure allowed antifreeze into one of the cylinders causing the failure. Oil analysis results are shown in Appendix 6.

\section{Operational Data}

Hennepin County has considerable experience with alcohol fuels including storage and dispensing systems. We have used gasohol since 1983, and experienced all the early system problems inherent with alcohol fuels. In 1986, the county replaced the storage tanks as well as the pumping equipment. Gasohol had been in these tanks exclusively until the E95 demonstration when the county converted one tank solely to E95. This demonstration project lasted 3 years, and we did not have any of the fuel dispensing problems that we had experienced in the early 
days of gasohol use. We did not replace any storage or pumping components throughout the project term, nor were there any reports from employees of any problems related to the E95.

Ethanol fuel is as easy to use as any conventional petroleum fuel. For Hennepin County, the conversion could not have been easier. There were no capital costs and no lengthy construction or installation projects. In fact, from the drivers' point of view, the larger fuel tank, fill time, and different fuel smell are the only differences they noticed at the pump.

In terms of cold weather fueling, E95 performed better than diesel. The county has experienced, during extreme cold weather, some gelling problems when fueling with diesel, which was not evident with E95. Hennepin County did not experience any cold starting problems with E95 because all the snow plow units were stored in heated garages.

\section{Safety}

The county did not experience any safety incidents with E95. Because it is not extremely toxic even in the denatured state (95\% ethyl alcohol, $5 \%$ unleaded gasoline), it does not require any precautions beyond those needed for gasoline.

\section{Emissions}

West Virginia University (WVU) tested the emissions of the trucks twice during the project. The WVU transportable heavy-duty truck dynamometer was brought on site to test the emissions of the ethanol trucks as well as the diesel control truck.

The data generally show that oxides of nitrogen are slightly lower for the ethanol vehicles than for the diesel vehicle, and that particulate matter levels are significantly lower for ethanol. The diesel emissions levels remained consistent from year to year, and offered lower carbon monoxide emissions than the ethanol trucks, whereas the carbon monoxide emissions from ethanol \#2 rose by a factor of three between 1994 and 1995. It is possible that catalytic converter efficiency had degraded on this vehicle, because hydrocarbon emissions also rose. Although the diesel vehicle had lower hydrocarbon emissions than the ethanol vehicles, it was evident that unburned ethanol was present as a significant fraction of the total hydrocarbon emissions of the ethanol vehicles. However, because oxides of nitrogen and particulate matter may be considered the emissions of concern for the heavy-duty fleet, the ethanol-powered vehicles have an advantage over their diesel counterparts relative to emissions. The complete WVU test results and summary are in Appendices 7 and 8. 


\section{Conclusions}

This project was successful in several ways. First, a heavy-duty engine was developed and emissions certified to run on a high percentage blend of ethanol, a domestically produced and renewable fuel, for use in urban transit buses and over-the-road trucks. To date, the DDC 6V92TA is the only alcohol fuel engine that can make this claim. Second, the ethanol trucks successfully performed the same rigorous duties as the diesel control trucks in a demanding vocation and harsh winter environment. Third, with some refinement, ethanol engines could prove to be as reliable and durable as their diesel counterparts. From a functional standpoint, the fuel filter clogging issue was the only significant barrier from the fleet administrator and the drivers' viewpoint. Fourth and finally, although E95 trucks emitted more hydrocarbons and carbon monoxide than the diesel trucks, the oxides of nitrogen and particulate matter, the emissions of concern for heavy-duty vehicles, were reduced.

The real hurdles for the widespread use of high percentage blends of ethanol are fuel costs, vehicle range, and infrastructure. Although exact fuel costs will vary by region and tax status, the E95 cost more than three times as much as diesel \#2 (on an equivalent energy basis) in this demonstration. The vehicle range was an issue, even in this application where the vehicles covered a relatively small area. With this in mind, over-the-road travel would be impossible without an extensive fueling infrastructure. 


\section{Appendix 1 \\ Collection Form to Gather Data: Driver Option}


Appendix. 1: Data Collection Form to Gather Driver Option

hicle Weekly Log Sheet

erator:

Week of

one:

) $-$

Fuel Cost Per Gallon

Janization:

jicle ID \#

ense \#

ta Collection for Week

ginning (Monday): $/ 1993$

\begin{tabular}{l|l|l|l|l|l|l|l|l|} 
& Monday & Tuesday & Wednesday Thursday & Friday & Sunday \\
\hline Starting Odometer Reading: & & & & & & & \\
\hline Ending Odometer Reading: & & & & & & & & \\
\hline
\end{tabular}

Please evaluate the performance of your vehicle by responding to the pertomance categories listed in the table below.

Responses are: S= Superior; $S=$ No Problem; $A=$ Mildly Annoying; $T=$ Very Troublesome

\begin{tabular}{|c|c|c|c|c|c|c|c|}
\hline & Monday & Tuesday & Wedresday & Thursday & Friday & Saturday & Sunday \\
\hline \multicolumn{8}{|l|}{ Hard to Stant } \\
\hline \multicolumn{8}{|c|}{ Stalled After Starting } \\
\hline \multicolumn{8}{|c|}{ Stalled in Tratfic } \\
\hline \multicolumn{8}{|l|}{ Idle Quality } \\
\hline \multicolumn{8}{|c|}{ Acceleration Quatity } \\
\hline \multicolumn{8}{|c|}{ Hesitation. Coughing } \\
\hline Lack of Power & & & & & & & \\
\hline
\end{tabular}

Regardless of severity, if any problem occurred. describe the type of efiving (e.g.. city. freoway), time of occurrence, and approximate weather condition (temp/moisture) for each problem occurrence.

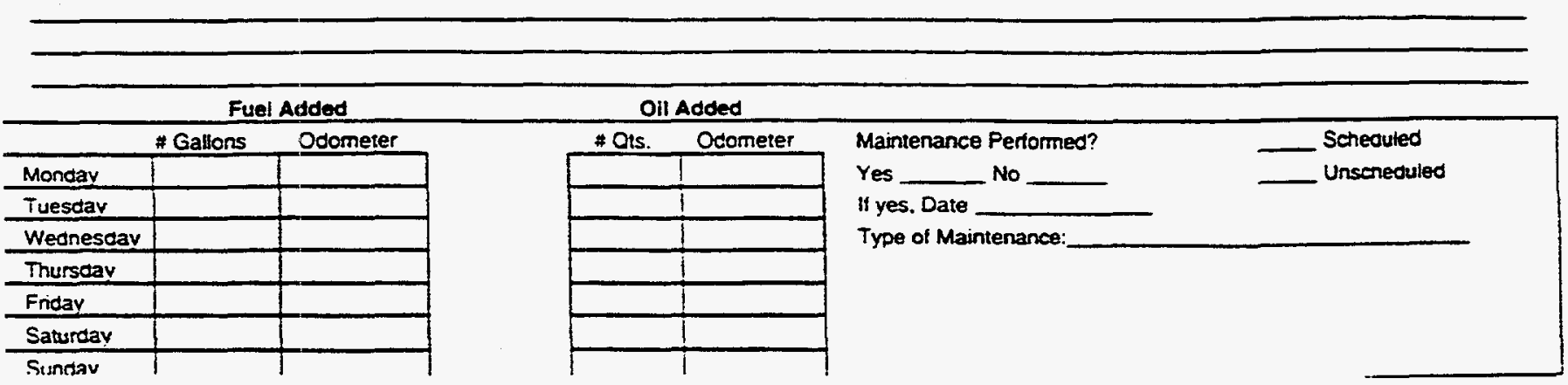




\section{Appendix 2 \\ Map of Demonstration Area}




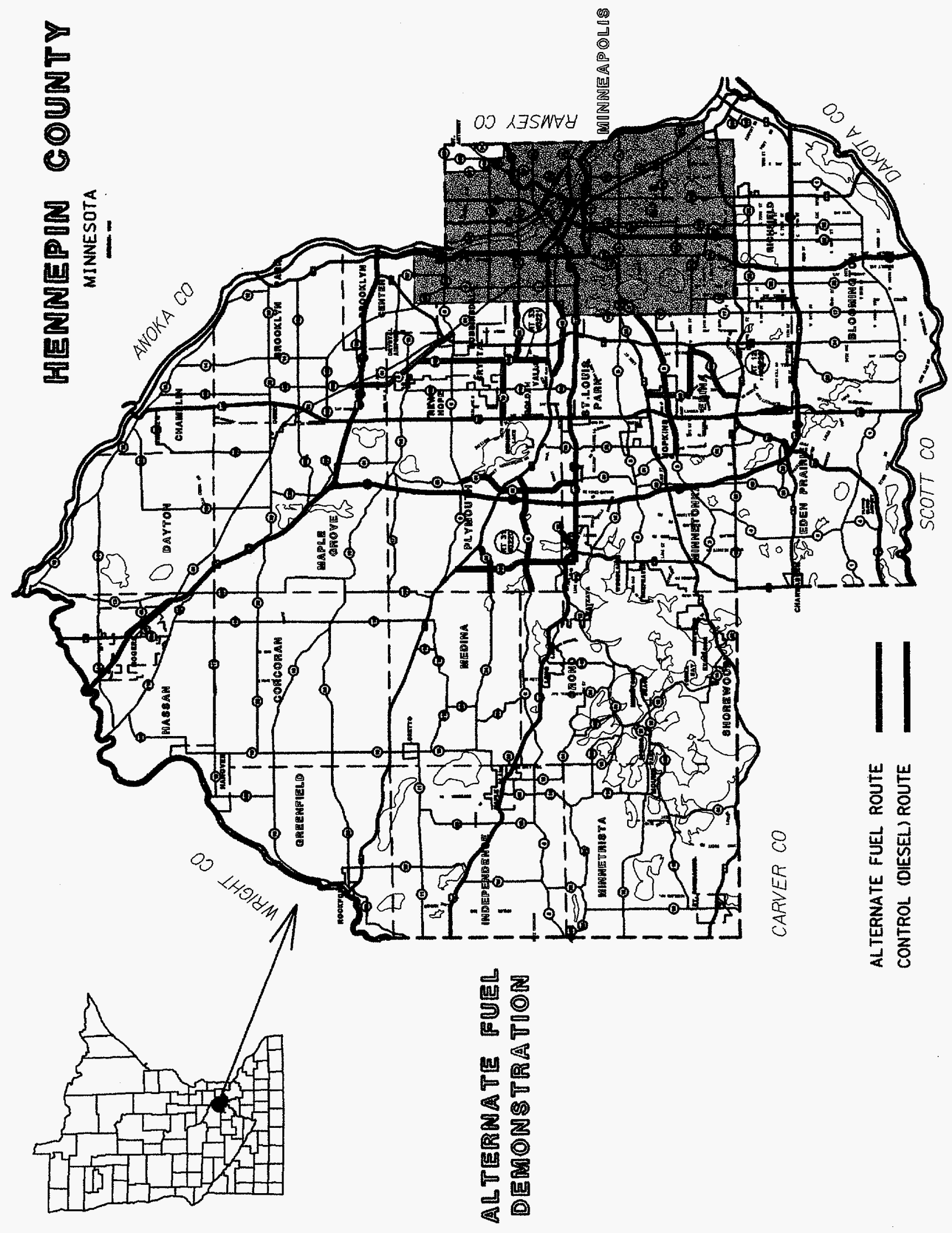




\section{Appendix 3 \\ Specifications for E95 Ethanol Fuel}


SPECIFICATIONS FOR E95 ETHANOL FUEL

Interstate Detroit Diesel, Inc.

$\begin{array}{lll}\text { Specification } & \text { Value } & \text { Test Method } \\ \begin{array}{l}\text { Ethanol } \\ \text { Denatured fuel } \\ \text { ethanol }\end{array} & 92 \text { vol. \% (min.) } & \text { ASTM D 3545-90 } \\ \begin{array}{l}\text { Other alcohols and } \\ \text { others }\end{array} & 98 \text { vol. \% (min.) } \\ \begin{array}{l}\text { Hydrocarbons } \\ \text { gasoline or diesel } \\ \text { fuel derived } \\ \text { then subtract concentration of alcohols, ethers, and water from 100 to obtain percent } \\ \text { hydrocarbons. }\end{array} & \text { ASTM D 4815-89 } \\ & 5 \text { mass \% (max.) }\end{array}$

Acidity as acetic acid

0.007 mass $\%(\max$.

ASTM D 1613-85

Total chlorine as chloride

0.0004 mass $\%(\max$.

ASTM D 3120-87 modified for organic chlorides, and ASTM 2988-86.

Copper

$0.07 \mathrm{mg} / 1$ (max.)

ASTM D 1688-90 as modified in ASTM D 4806-88.

Lead

$2 \mathrm{mg} / \mathrm{l}(\max$.

ASTM D 3237-90

Phosphorus

$0.2 \mathrm{mg} / 1(\max$.

ASTM D 3231-89

Sulfur

0.01 mass $\%(\max$.

ASTM D 3120-87

Gum, heptane washed

$5 \mathrm{mg} /$ liter (max.)

ASTM D 381-86

Total particulates

$5 \mathrm{mg} / 1$ (max.)

ASTM D 2276-89

Water content

0.5 mass \% (max.)

ASTM E 203-75

Appearance

Free of turbidity suspended matter and sediment

Visually determined at 15 degrees $\mathrm{C}$ by Proc. A of ASTM D 4176-86

Option: 0.06\% (8 oz. per 100 gal) Lubrizol, Part \#23509970 
1 Must meet and ASTM D 4806-88 specification for denatured fuel ethanol, except the denaturant must be representative of unleaded gasoline that is commercially available blended at a maximum of 4 to 5 parts gasoline to 100 parts by volume fuel ethanol (including water) to form the denatured fuel ethanol. The final blend specifications for E-100 fuel take precedence over the ASTM D 4806-88 specification. 


\section{Appendix 4 \\ Maintenance Costs by Truck System and Year}


Repair Detail, Truck Number 3221

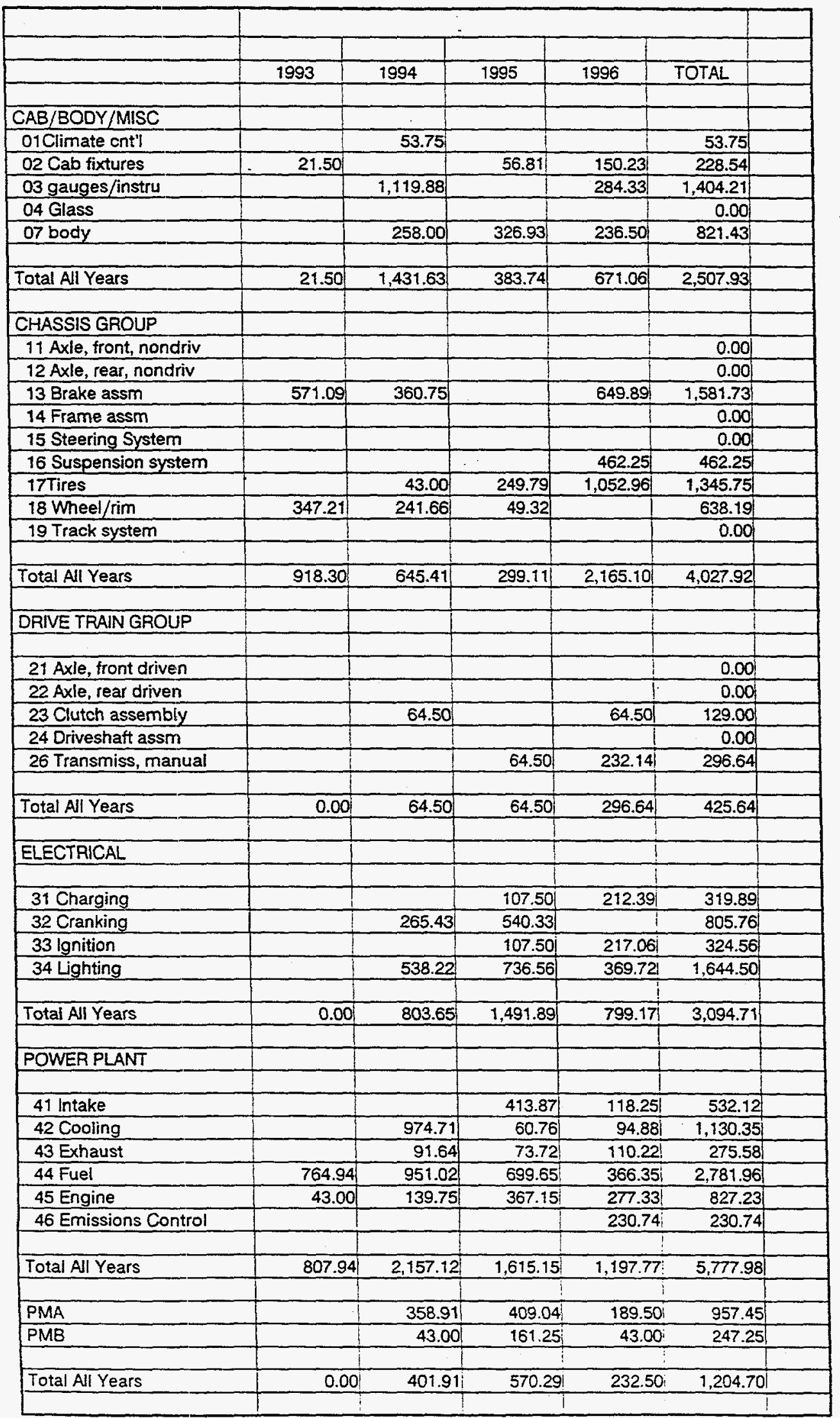


Repair Detail, Truck Number 3221

\begin{tabular}{|c|c|c|c|c|c|}
\hline & & & & & \\
\hline & & & & & \\
\hline & 1993 & 1994 & 1995 & 1996 & TOTAL \\
\hline 51 Access general & 11879 & 180259 & 29025 & & \\
\hline 52 Access electric & $\frac{110.19}{96.75}$ & $\frac{1,802.59}{849.57}$ & $\frac{290.25}{448.00}$ & $\frac{5 / 3.4 !}{107.50}$ & $\frac{2,185.10}{1,501.82}$ \\
\hline 54 Snow equip & & 150.50 & 43.00 & & 193.50 \\
\hline 55 Lift systems & & & & & 0.00 \\
\hline 56 PTO systems & & & & & 0.00 \\
\hline 59 Veh coupling sys & 45.12 & & & & 45.12 \\
\hline Total All Years & 260.66 & $2,802.66$ & 781.25 & 680.97 & $4,525.54$ \\
\hline \multirow{2}{*}{\multicolumn{6}{|c|}{ SPECIAL APPLICAT }} \\
\hline & & & & & \\
\hline 61 Buckets & & & & & 0.00 \\
\hline 62 Blades & & & & & 0.00 \\
\hline 63 Brooms & & & & & 0.00 \\
\hline 64 Cables & & & & & 0.00 \\
\hline 65 Heating units & & & & & 0.00 \\
\hline 66 Special quip & 238.14 & 129.00 & 179.34 & 64.50 & 610.98 \\
\hline Total All Years & 238.14 & 129.00 & 179.34 & 64.50 & 610.98 \\
\hline & & & & & \\
\hline \multicolumn{6}{|l|}{ HYDRAULICS } \\
\hline & & & & & \\
\hline 71 Hydraulic pump & & & & 532.88 & 532.88 \\
\hline 72 Hydraulic cylinder & & & & & 0.00 \\
\hline 73 Lines & & 172.00 & 43.00 & & 215.00 \\
\hline 74 Valves & & & 1 & & 0.00 \\
\hline 75 Motors & & & 1 & & 0.00 \\
\hline 76 Couplings & & 43.00 & 21.50 & & 64.50 \\
\hline 77 Tank assm & & 159.11 & 38.74 & 27.99 & 225.84 \\
\hline 78 Auxiliary & & & 1 & & 0.00 \\
\hline \multirow{3}{*}{ Total All Years } & & & 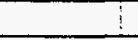 & & \\
\hline & 0.00 & 374.11 & 103.24 & 560.87 & $1,038.22$ \\
\hline & & & $!$ & & \\
\hline \multirow[t]{2}{*}{ MISC EQUIP NORK } & & & 1 & & \\
\hline & & & $i$ & & \\
\hline 91 Misc Tank & & & 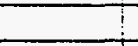 & & 0.00 \\
\hline 92 Misc cutting equip & & & 1 & & 0.00 \\
\hline 98 Misc inside work & $4,312.27$ & 594.83 & 919.83 & 702.21 & $6,529.14$ \\
\hline 99 Outside vendor & & 183.37 & 160.08 & 326.60 & 670.05 \\
\hline 9830 New veh prep & 4312.27 & & & & \\
\hline Total All Yrs-Veh Prep & 0.00 & 778.20 & $1,079.91$ & $1,028.81$ & $2,886.92$ \\
\hline & & & $!$ & & \\
\hline \multirow[t]{14}{*}{ Total A!! Years } & & & $!$ & & $7,199.15$ \\
\hline & & & 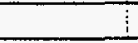 & & \\
\hline & & & GRAND & OTAL & $30,412.81$ \\
\hline & & & & & \\
\hline & & & 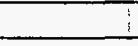 & & \\
\hline & & & $\because$ & & \\
\hline & & & & & \\
\hline & & & 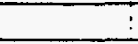 & & \\
\hline & & & $\vdots$ & & \\
\hline & & & & & \\
\hline & & & & & \\
\hline & & & & & \\
\hline & & & & & \\
\hline & 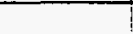 & & & & \\
\hline
\end{tabular}


Repair Detail, Truck Number 3228

\begin{tabular}{|c|c|c|c|c|c|c|}
\hline \\
\hline & & & & & & \\
\hline & & & & & & \\
\hline & 1993 & 1994 & 1995 & 1996 & TOTAL & \\
\hline & & & & & & \\
\hline \multicolumn{7}{|l|}{ CAB/BODY/MISC } \\
\hline 01Climate cnt"l & & 118.69 & & & 118.69 & \\
\hline 02 Cab fixtures & & 77.92 & 43.00 & 86.00 & 206.92 & \\
\hline 03 gauges/instru & & 251.81 & 227.95 & 134.53 & 614.29 & \\
\hline 04 Glass & & & & & 0.00 & \\
\hline 07 body & 215.02 & & & 356.48 & 571.50 & \\
\hline Total Al Years & 215.02 & 448.42 & 270.95 & 577.01 & $1,511.40$ & \\
\hline & & & & & & \\
\hline \multicolumn{7}{|l|}{ CHASSIS GROUP } \\
\hline 11 Axle, front, nondriv & & & 140.04 & 43.00 & 183.04 & \\
\hline 12 Axle, rear, nondriv & & & & & 0.00 & \\
\hline 13 Brake assm & 75.25 & 389.77 & 97.85 & 140.88 & 703.75 & \\
\hline 14 Frame assm & & & & & 0.00 & \\
\hline 15 Steering System & & & & & 0.00 & \\
\hline 16 Suspension sysiem & & & & & 0.00 & \\
\hline 17 Tires & & & 353.62 & & 353.62 & \\
\hline 18 Wheel/rim & & 166.41 & & & 166.41 & \\
\hline 19 Track system & & & & & 0.00 & \\
\hline & & & & & & \\
\hline Total All Years & 75.25 & 556.18 & 591.51 & 183.88 & $1,406.82$ & \\
\hline & & & & & & \\
\hline \multicolumn{7}{|l|}{ DAIVE TRAIN GROUP } \\
\hline & & & & & & \\
\hline 21 Axle, front driven & & & & & 0.00 & \\
\hline 22 Axle, rear driven & & 96.75 & & & 96.75 & \\
\hline 23 Clutch assembly & & & & 21.50 & 21.50 & \\
\hline 24 Driveshaft assm & & & & & 0.00 & \\
\hline 26 Transmiss, manual & & & 86.00 & & 86.00 & \\
\hline Total All Years & 0.00 & 96.75 & 86.00 & 21.501 & 204.25 & \\
\hline & & & & $!$ & & \\
\hline \multicolumn{7}{|l|}{ ELECTRICAL } \\
\hline & & & & & & \\
\hline 31 Charging & & 391.40 & 86.00 & 37.28 & 514.68 & \\
\hline 32 Cranking & & 379.53 & 303.83 & 223.77 & 907.13 & \\
\hline 33 Ignition & & & & 347.88 & 347.88 & \\
\hline 34 Lighting & & 581.42 & 899.20 & 303.99 & $1,784.61$ & \\
\hline & & & & 1 & & \\
\hline Total All Years & 0.00 & $1,352.35$ & $1,289.03$ & 912.92 & $3,554.30$ & \\
\hline & & & & & & \\
\hline \multicolumn{7}{|l|}{ POWER PLANT } \\
\hline 41 Intake & & & & 121.41 & 121.41 & \\
\hline 42 Cooling & 21.50 & 350.39 & 30.38 & 248.73 & 651.00 & \\
\hline 43 Exhaust & & 127.47 & & $423.96 i$ & 551.43 & \\
\hline 44 Fuel & 344.88 & 943.55 & 353.84 & 981.12 & $2,623.39$ & \\
\hline 45 Engine & & 483.75 & & $i$ & 483.75 & \\
\hline \multirow[t]{2}{*}{46 Emissions Control } & 35.70 & & & 1 & 35.70 & \\
\hline & & 1 & & $!$ & & \\
\hline \multirow[t]{2}{*}{ Total All Years } & 402.08 & 1.905 .16 & 384.22 & $1,775.22 !$ & 4.466 .68 & \\
\hline & & & 1 & 1 & & \\
\hline PMA & & 135.82 & 185.89 & $153.65 \mathrm{i}$ & 475.36 & \\
\hline \multirow[t]{2}{*}{ PMB } & & 193.50 & 193.50 & $86.00 !$ & 473.00 & \\
\hline & & & & $!$ & - & \\
\hline Total All Years & 0.00 & 329.32 & 379.39 & $239.65 i$ & 948.36 & \\
\hline
\end{tabular}


Repair Detail, Truck Number 3228

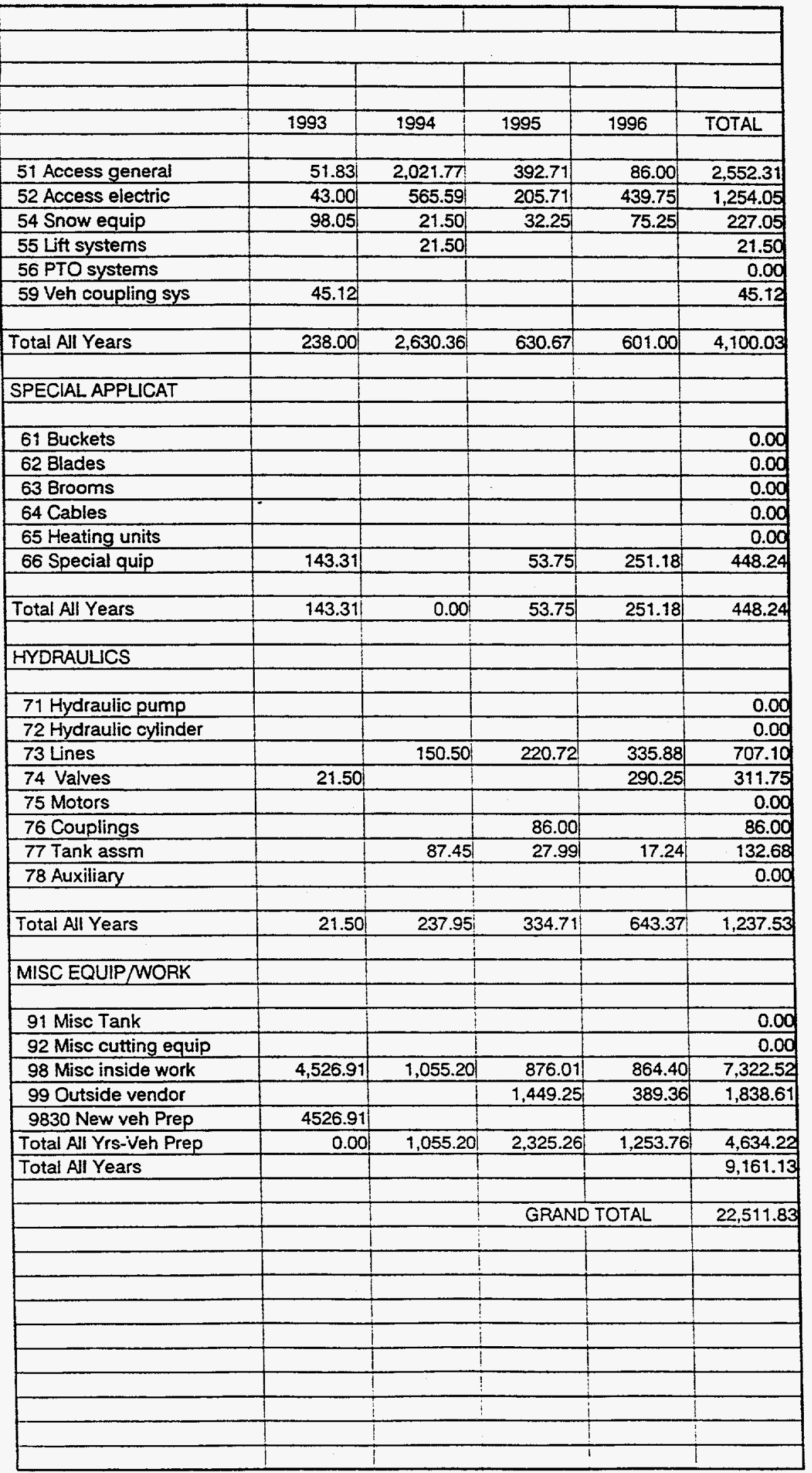




\begin{tabular}{|c|c|c|c|c|c|c|}
\hline & & & 1 & & & \\
\hline & & & & & & \\
\hline & 1993 & 1994 & 1995 & 1996 & TOTAL & \\
\hline & & & & & & \\
\hline \multicolumn{7}{|l|}{ CAB/BODY/MISC } \\
\hline 01Climate cnt'l & 0.00 & & 142.50 & & 142.50 & \\
\hline 02 Cab fixtures & 0.00 & & 23.16 & 310.00 & 333.16 & \\
\hline 03 gauges/instru & 0.00 & & 142.91 & 602.00 & 744.91 & \\
\hline 04 Glass & & & & & 0.00 & \\
\hline 07 body & 0.00 & 47.95 & 321.69 & 96.75 & 466.39 & \\
\hline Total All Years & 0.00 & 47.95 & 630.26 & $1,008.75$ & $1,686.96$ & \\
\hline & & & & & & \\
\hline \multicolumn{7}{|l|}{ CHASSIS GROUP } \\
\hline 11 Axje, front, nondriv & 0.00 & & & 21.50 & 21.50 & \\
\hline 12 Axle, rear, nondriv & 0.00 & & & & 0.00 & \\
\hline 13 Brake assm & 206.02 & & 692.79 & 179.02 & $1,077.83$ & \\
\hline 14 Frame assm & 0.00 & & & & 0.00 & \\
\hline 15 Steering Systern & 0.00 & & & & 0.00 & \\
\hline 16 Suspension system & 0.00 & & & 881.40 & 881.40 & \\
\hline 17Tires & 0.00 & 43.00 & 732.59 & $1,020.84$ & $1,796.43$ & \\
\hline 18 Wheel/rim & 347.21 & 155.66 & & & 502.87 & \\
\hline 19 Track system & 0.00 & & & & 0.00 & \\
\hline \begin{tabular}{|l} 
Total All Years \\
\end{tabular} & 553.23 & 198.66 & $1,425.38$ & $2,102.76$ & $4,280.03$ & \\
\hline & & & & & & \\
\hline \multicolumn{7}{|l|}{ DRIVE TRAIN GROUP } \\
\hline & & & & & & \\
\hline 21 Axle, front driven & & & & & 0.00 & \\
\hline 22 Axle, rear driven & & 43.00 & & & 43.00 & \\
\hline 23 Clutch assembly & & 129.00 & 53.75 & 21.50 & 204.25 & \\
\hline 24 Driveshaft assm & & & & & 0.00 & \\
\hline 26 Transmiss, manual & & 86.00 & 14.80 & & 100.80 & \\
\hline & & & & & & \\
\hline Total All Years & 0.00 & 258.00 & 68.55 & 21.50 & 348.05 & \\
\hline \multicolumn{7}{|l|}{ ELECTRICAL } \\
\hline & & & & & & \\
\hline 31 Charging & & 1 & & 193.31 & 193.31 & \\
\hline 32 Cranking & & 213.44 & & 421.33 & 634.77 & \\
\hline 33 Ignition & & 86.00 & & & 86.00 & \\
\hline 34 Lighting & & $1,107.53$ & 745.04 & 679.32 & $2,531.89$ & \\
\hline Total All Years & 0.00 & 1.406 .97 & 745.04 & $1,293.96$ & $3,445.97$ & \\
\hline \multicolumn{7}{|l|}{ POWER PLANT } \\
\hline 41 intake & & 161.38 & & & 161.38 & \\
\hline 42 Cooling & & 250.00 & 924.72 & $1,276.85$ & $2,451.57$ & \\
\hline 43 Exhaust & 62.09 & & 21.50 & & 83.59 & \\
\hline 44 Fuel & & 33.62 & 36.94 & 134.69 & 205.25 & \\
\hline 45 Engine & & 43.00 & & & 43.00 & \\
\hline 46 Emissions Control & & & & & 0.00 & \\
\hline \multirow[t]{2}{*}{ Total All Years } & 62.09 & 488.00 & 983.16 & $1,411.54$ & $2,944.79$ & \\
\hline & & & 1 & & $2=0.14$ & \\
\hline PMA & 180.51 & 335.36 & 368.96 & 165.72 & 1.050 .55 & \\
\hline \multirow[t]{2}{*}{ PMB } & & 43.90 & 32.25 & 21.50 & 97.65 & \\
\hline & & $!$ & $!$ & & $i$ & \\
\hline \multirow[t]{2}{*}{ Total All Years } & 180.51 & 379.26 & 401.21 & 187.22 & $1,148.20$ & \\
\hline & & & $\vdots$ & & I & \\
\hline
\end{tabular}


Repair Detail, Truck Number 3220

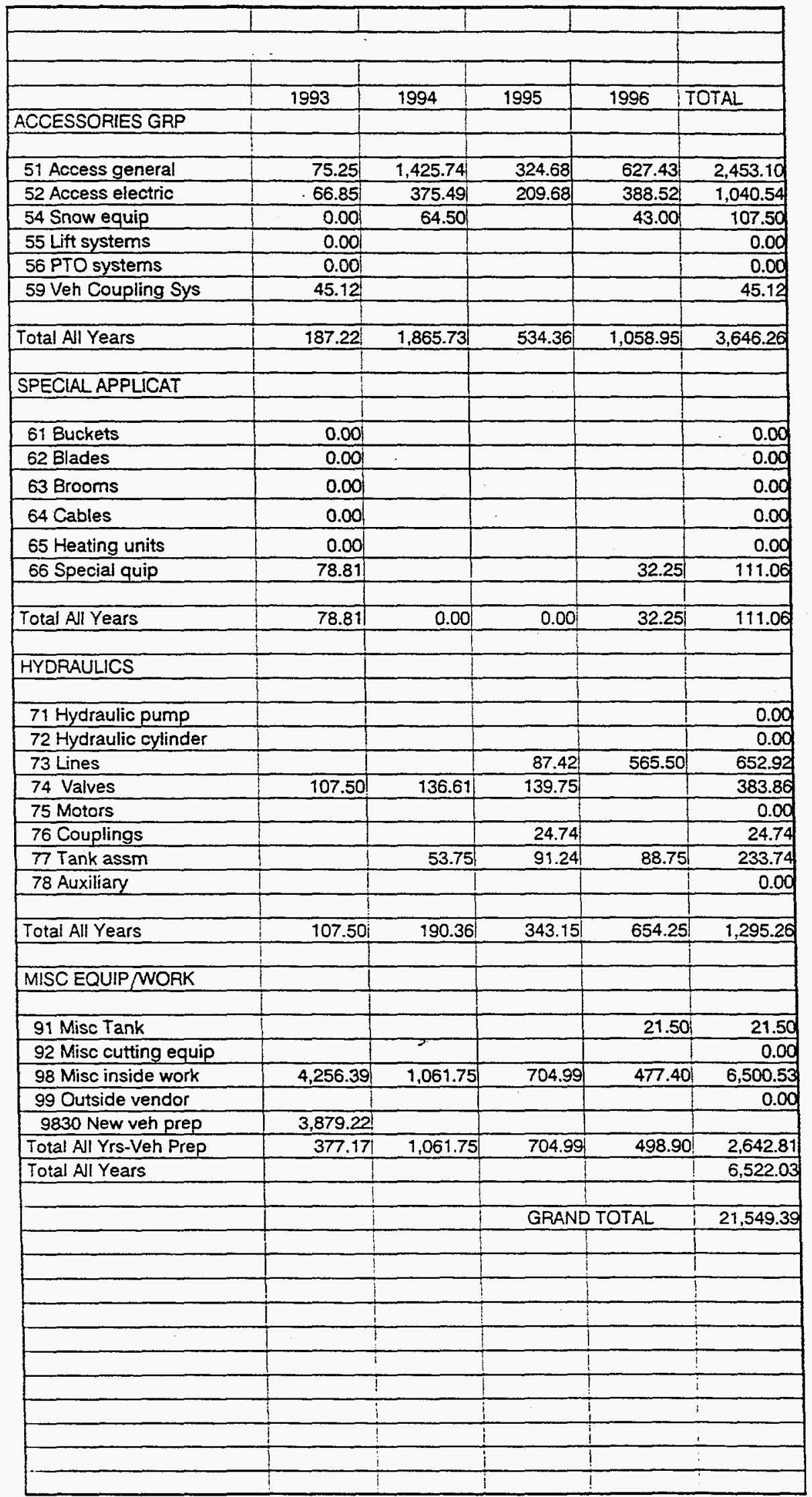


Appendix 5

Repair History by Truck System 
SYNCSORT 3.6ARI TPF2B US PATENTS: 4210961,5117495, OTHER PAT. PEND. (C) SYNCSORT INC. 1993 DATE=96/324 TIME $=14.59 .13$ PARMLIST : HENNEPIN COUNTY INFORMATION SERVICES ESA 5.2 .2 CPU MODEL 3090

SORT SIZE=E00028 04, FIELDS $=101,04, \mathrm{CH}, \mathrm{A}, 067,04, \mathrm{CH}, \mathrm{A}, 05,01, \mathrm{CH}, \mathrm{A}, 091,04, \mathrm{CH}, \mathrm{A}, 067,08$

$$
\text { 2D.A) }
$$

RECORD TYPE=F LENGTH $=00148$ 


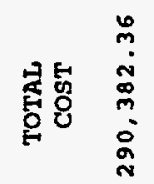

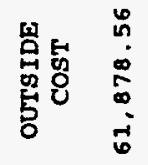

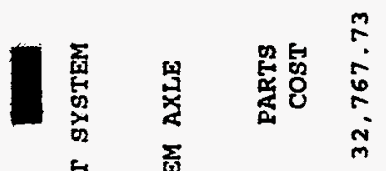

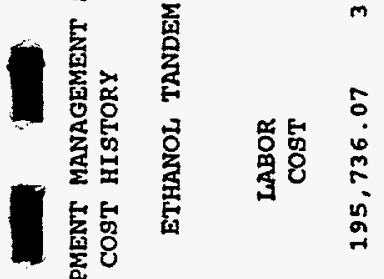

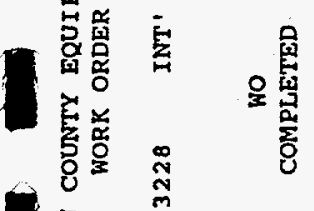

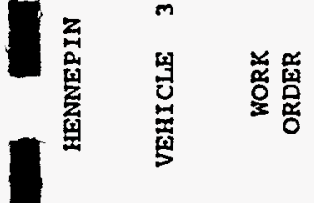

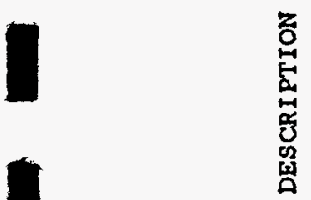

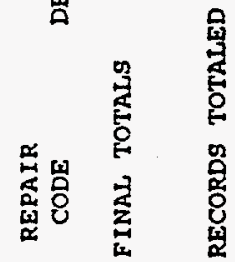

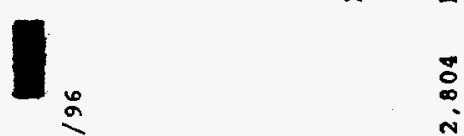


VEHICLE 3228 INT' ETHANOL TANDEM AXLE

\begin{tabular}{|c|c|c|c|c|c|c|c|}
\hline $\begin{array}{l}\text { REPAIR } \\
\text { CODE }\end{array}$ & DESCRIPTION & $\begin{array}{l}\text { WORK } \\
\text { ORDER }\end{array}$ & $\begin{array}{c}\text { WO } \\
\text { COMPLETED }\end{array}$ & $\begin{array}{r}\text { LABOR } \\
\text { COST }\end{array}$ & $\begin{array}{r}\text { PARTS } \\
\text { COST }\end{array}$ & $\begin{array}{c}\text { OUTSIDE } \\
\text { CosT }\end{array}$ & $\begin{array}{l}\text { TOTAL } \\
\text { COST }\end{array}$ \\
\hline & ENDYR TOTAI. & & & 0.740 .75 & 269.48 & 1.343 .51 & $6,353.74$ \\
\hline & UNIT TOTAL & & & $19,136.75$ & $1,655.75$ & $6,246.24$ & $27,038.74$ \\
\hline
\end{tabular}




REPAIR
CODE
7301R HESCRIPTION
REPAIR TYPE TOTAL
REPAIR TYPE TOTAL

REPAIR SUB GROUP TOTAL

74010 HYDRAULIC VALVE REPAIR TYPE TOTAL 74760 CABLE, CONTL LEVER
74760 CABLE, CONTL LEVER REPAIR TYPE TOTAL

REPAIR SUB GROUP TOTAL

7721R HYD TANK FILTER REPAIR TYPE TOTAL.

REPAIR SUB GROUP TOTAL

REPAIR GROUP TOTAL

\begin{tabular}{|c|c|}
\hline 98200 & GENERAI, GREASINO \\
\hline 98200 & GENERAL GREASING \\
\hline 98200 & GENERAL GREASING \\
\hline 98200 & GENERAL GREASINC \\
\hline 98200 & GENERAL GREASING \\
\hline
\end{tabular}

98601 TRUCK INSPECTION REPAIR TYPE TOTAL

98900 DIAGNOSTIC ELECTRIC REPAIR TYPE TOTAL

98950 DIAGNOSTIC MECHANICL.

98951 DIAGNOSTIC MECHANICL

98951 DIAGNOSTIC MECHANICL REPAIR TYPE TOTAL

REPAIR SUB GROUP TOTAL

99400 POWER PLANT GROUP REPAIR TYPE TOTAL

259385

M08225

M08407

M08607

M09175

WORK ORDER COST HISTORY

VEHICLE 3228 INT' ETHANOL TANDEM AXLE

WORK
ORDER

WO
COMPLETED

$259106 \quad 1996 / 03 / 20$

257703

$1996 / 01 / 30$

257703

$1996 / 01 / 30$

$1996 / 07 / 02$

0298

262379

257310

257310

261019

$1996 / 09 / 17$

REPAIR SUB GROUP TOTAL,

REPAIR GROUP TOTAL

PM/MINUS PM TOTAL

\begin{tabular}{|c|c|c|c|}
\hline $\begin{array}{r}\text { LABOR } \\
\text { COST }\end{array}$ & $\begin{array}{r}\text { PARTS } \\
\text { Cost }\end{array}$ & $\begin{array}{l}\text { OUTSIDE } \\
\text { COST }\end{array}$ & $\begin{array}{l}\text { TOTAL } \\
\text { COST }\end{array}$ \\
\hline 322.50 & 13.38 & .00 & 335.88 \\
\hline 322.50 & 13.38 & .00 & $335.8 \mathrm{a}$ \\
\hline 322.50 & 13.38 & .00 & 335.88 \\
\hline 43.00 & .00 & .00 & 43.00 \\
\hline 43.00 & .00 & .00 & 43.00 \\
\hline 75.25 & .00 & .00 & 75.25 \\
\hline 172.00 & .00 & .00 & 172.00 \\
\hline 247.25 & .00 & .00 & 247.25 \\
\hline 290.25 & .00 & .00 & 290.25 \\
\hline 10.75 & 6.49 & .00 & 17.24 \\
\hline 10.75 & 6.49 & .00 & 17.24 \\
\hline 10.75 & 6.49 & .00 & 17.24 \\
\hline 623.50 & 19.87 & .00 & 643.37 \\
\hline 96.75 & .88 & .00 & 97.63 \\
\hline 86.00 & .88 & .00 & 86.88 \\
\hline 86.00 & .88 & .00 & 86.88 \\
\hline 86.00 & .88 & .00 & 86.88 \\
\hline 86.00 & .88 & .00 & 86.88 \\
\hline 440.75 & 4.40 & .00 & 445.15 \\
\hline 43.00 & .00 & .00 & 43.00 \\
\hline 43,00 & .00 & .00 & 43.00 \\
\hline 129.00 & .00 & .00 & 129.00 \\
\hline 129.00 & .00 & .00 & 129.00 \\
\hline 64.50 & .00 & .00 & 64.50 \\
\hline 21.50 & .00 & .00 & 21.50 \\
\hline 64.50 & .00 & .00 & 64.50 \\
\hline 150.50 & .00 & .00 & 150.50 \\
\hline 763.25 & 4.40 & .00 & 767.65 \\
\hline .00 & .00 & 389.36 & 389.36 \\
\hline .00 & .00 & 389.36 & 389.36 \\
\hline .00 & .00 & 389.36 & 389.36 \\
\hline 763.25 & 4.40 & 389.36 & $1,157.01$ \\
\hline $4,515.00$ & 258.50 & $1,297.59$ & $6,071.09$ \\
\hline
\end{tabular}


VEHICLE 3228 INT' ETHANOI TANDEM AXLE

\begin{tabular}{|c|c|c|c|c|c|c|}
\hline $\begin{array}{l}\text { REPAIR } \\
\text { CODE }\end{array}$ & $\begin{array}{l}\text { HORK } \\
\text { ORDER }\end{array}$ & $\begin{array}{c}\text { HO } \\
\text { COMPLETED }\end{array}$ & $\begin{aligned} \text { LABOR } \\
\text { COST }\end{aligned}$ & $\begin{array}{l}\text { PARTS } \\
\text { COST }\end{array}$ & $\begin{array}{l}\text { OUTSIDE } \\
\text { COST }\end{array}$ & $\begin{array}{l}\text { TOTAL } \\
\text { COST }\end{array}$ \\
\hline FILTER， SECONDARY & 259385 & $1996 / 04 / 03$ & .00 & $=00$ & $4 \varepsilon .92$ & 46.32 \\
\hline 4411R FILTER, SECONDARY & 262379 & $1996 / 08 / 21$ & 21.50 & .00 & 145.42 & 166.92 \\
\hline REPAIR TYPE TOTAL & & & 21.50 & .00 & 192.34 & 213.84 \\
\hline FUEL LINES & 257194 & $1996 / 02 / 06$ & 118.25 & .00 & .00 & 118.25 \\
\hline REPAIR TYPE TOTAL & & & 118.25 & .00 & .00 & 118.25 \\
\hline $\begin{array}{l}\text { 44800 ELECTRONIC CONTROLS } \\
\text { REPAIR TYPE TOTAL }\end{array}$ & 257194 & $1996 / 02 / 06$ & $\begin{array}{l}193.50 \\
193.50\end{array}$ & $\begin{array}{l}14.43 \\
14.43\end{array}$ & $\begin{array}{l}.00 \\
.00\end{array}$ & $\begin{array}{l}207.93 \\
207.93\end{array}$ \\
\hline REPAIR SUB GROUP TOTAL & & & 580.50 & 21.89 & 378.73 & 981.12 \\
\hline REPAIR GROUP TOTAL & & & $1,096.50$ & 86.81 & 591.91 & $1,775.22$ \\
\hline MUD FLAP (s) & 262481 & $1996 / 08 / 15$ & 21.50 & .00 & .00 & 21.50 \\
\hline REPAIR TYPE TOTAL & & & 21.50 & .00 & .00 & 21.50 \\
\hline 51100 TARPALIN ASSEMBLY & 263644 & $1996 / 09 / 26$ & 21.50 & .00 & .00 & 21.50 \\
\hline TARPALIN ASSEMBLY & 263919 & $1996 / 10 / 21$ & 43.00 & .00 & .00 & 43.00 \\
\hline REPAIR TYPE TOTAL & & & 64.50 & .00 & .00 & 64.50 \\
\hline REPAIR SUB GROUP TOTAL & & & 86.00 & .00 & .00 & 86.00 \\
\hline 52400 BOX SHAKER & 257194 & $1996 / 02 / 06$ & 96.75 & .63 & .00 & 97.38 \\
\hline REPAIR TYPE TOTAL & & & 96.75 & .63 & .00 & 97.38 \\
\hline MISC WIRING & 258020 & $1996 / 02 / 16$ & 225.75 & .00 & 19.87 & 245.62 \\
\hline MISC WIRING & 263344 & $1996 / 09 / 17$ & 96.75 & .00 & .00 & 96.75 \\
\hline REPAIR TYPE TOTAL & & & 322.50 & .00 & 19.87 & 342.37 \\
\hline REPAIR SUB GROUP TOTAL & & & 419.25 & .63 & 19.87 & 439.75 \\
\hline 54020 SNOW PLOW BLADE & 257310 & $1996 / 01 / 23$ & 75.25 & .00 & .00 & 75.25 \\
\hline REPAIR TYPE TOTAL & & & 75.25 & .00 & .00 & 75.25 \\
\hline REPAIR SUB GROUP TOTAL & & & 75.25 & .00 & .00 & 75.25 \\
\hline REPAIR GROUP TOTAL & & & 580.50 & .63 & 19.87 & 601.00 \\
\hline SP EQUIP ACCESSORIES & 260236 & $1996 / 05 / 07$ & 161.25 & 5.15 & .00 & 166.40 \\
\hline 66000 SP EQUIP ACCESSORIES & 252154 & $1996 / 07 / 02$ & .00 & .00 & 20.28 & 20.28 \\
\hline 6600L SP EQUIP ACCESSORIES & 262523 & $1996 / 08 / 15$ & 21.50 & .00 & .00 & 21.50 \\
\hline SP EQUIP ACCESSORIES & 264085 & $1996 / 10 / 23$ & 43.00 & .00 & .00 & 43.00 \\
\hline REPAIR TYEE TOTAL & & & 225.75 & 5.15 & 20.28 & 251.18 \\
\hline REPAIR SUB GROUP TOTAL & & & 225.75 & 5.15 & 20.28 & 251.18 \\
\hline REENIR GROUP TOTAL, & & & 225.75 & 5.15 & 20.28 & 251.18 \\
\hline
\end{tabular}


VEHICLE 3228 INT' ETHANOL TANDEM AXLE

\begin{tabular}{|c|c|c|c|c|c|c|}
\hline $\begin{array}{l}\text { REPAIR } \\
\text { CODE }\end{array}$ & $\begin{array}{l}\text { WORK } \\
\text { ORDER }\end{array}$ & $\begin{array}{c}\text { WO } \\
\text { COMPLETED }\end{array}$ & $\begin{array}{l}\text { LABOR } \\
\text { COST }\end{array}$ & $\begin{array}{l}\text { PARTS } \\
\text { COST }\end{array}$ & $\begin{array}{l}\text { OUTSIDE } \\
\text { COST }\end{array}$ & $\begin{array}{l}\text { TOTAL } \\
\text { COST }\end{array}$ \\
\hline $\begin{array}{l}34500 \text { SANDER/WORK LAMP } \\
\text { REPAIR TYPE TOTAL }\end{array}$ & 257860 & $1996 / 02 / 06$ & $\begin{array}{l}21.50 \\
21.50\end{array}$ & $\begin{array}{l}.00 \\
.00\end{array}$ & $\begin{array}{l}.00 \\
.00\end{array}$ & $\begin{array}{l}21.50 \\
21.50\end{array}$ \\
\hline $\begin{array}{l}34700 \text { FUSES, LIGHT(S) } \\
\text { REPAIR TXPE TOTAL }\end{array}$ & 258720 & $1996 / 03 / 11$ & $\begin{array}{l}43.00 \\
43.00\end{array}$ & $\begin{array}{l}1.47 \\
1.47\end{array}$ & $\begin{array}{l}.00 \\
.00\end{array}$ & $\begin{array}{l}44.47 \\
44.47\end{array}$ \\
\hline FLASHER UNIT & 260369 & $1996 / 05 / 14$ & 21.50 & 12.68 & .00 & 34.18 \\
\hline REPAIR TYPE TOTAL & & & 21.50 & 12.68 & .00 & 34.18 \\
\hline REPAIR SUB GROUP TOTAL & & & 182.75 & 78.00 & 43.24 & 303.99 \\
\hline REPAIR GROUP TOTAL & & & 612.75 & 119.02 & 181.15 & 912.92 \\
\hline$\$ 1300$ SUPERCHARGER & 257310 & $1996 / 02 / 23$ & 86.00 & .00 & 35.41 & 121.41 \\
\hline REPAIR TYPE TOTAL & & & 86.00 & .00 & 35.41 & 121.41 \\
\hline REPAIR SUB GROUP TOTAL & & & 86.00 & .00 & 35.41 & 121.41 \\
\hline COOLING SYSTEM & 260369 & $1996 / 05 / 14$ & 64.50 & .00 & .00 & 64.50 \\
\hline COOLING SYSTEM & 262379 & $1996 / 08 / 21$ & .00 & .00 & 7.96 & 7.96 \\
\hline REPAIR TYPE TOTAL & & & 64.50 & .00 & 7.96 & 72.46 \\
\hline RADIATOR & 262379 & $1996 / 08 / 21$ & 43.00 & .00 & .00 & 43.00 \\
\hline REPAIR TYPE TOTAL & & & 43.00 & .00 & .00 & 43.00 \\
\hline $\begin{array}{l}\text { 4212R COOLANT FILTER } \\
\text { REPAIR TYPE TOTAL }\end{array}$ & 262379 & $1996 / 08 / 21$ & $\begin{array}{l}10.75 \\
10.75\end{array}$ & $\begin{array}{l}8.88 \\
8.88\end{array}$ & $\begin{array}{l}.00 \\
.00\end{array}$ & $\begin{array}{l}19.63 \\
19.63\end{array}$ \\
\hline 4231R WATER PUMP BELT & 262379 & $1996 / 08 / 21$ & 64.50 & .00 & 49.14 & 113.64 \\
\hline REPAIR TYPE TOTAL & & & 64.50 & .00 & 49.14 & 113.64 \\
\hline REPAIR SUB GROUP TOTAL & & & 182.75 & 8.88 & 57.10 & 248.73 \\
\hline EXT MANIFOLD GASKET & 258766 & $1996 / 03 / 28$ & 43.00 & .00 & .00 & 43.00 \\
\hline REPAIR TYPE TOTAL & & & 43.00 & .00 & .00 & 43.00 \\
\hline EXHAUST TUBING & 262379 & $1996 / 08 / 21$ & 204.25 & 56.04 & 120.67 & 380.96 \\
\hline REPAIR TYPE TOTAL & & & 204.25 & 56.04 & 120.67 & 380.96 \\
\hline REPAIR SUB GROUP TOTAL & & & 247.25 & 56.04 & 120.67 & 423.96 \\
\hline FUEL SYSTEM & 257310 & $1996 / 01 / 23$ & 129.00 & 7.46 & .00 & 136.46 \\
\hline FUEL SYSTEM & 259385 & $1996 / 04 / 03$ & 64.50 & .00 & .00 & 64.50 \\
\hline REPAIR TYPE TOTAL & & & 193.50 & 7.46 & .00 & 200.96 \\
\hline FILTER, PRIMARY & 257194 & $1996 / 02 / 06$ & 32.25 & .00 & 63.97 & 96.22 \\
\hline FILTER, PRIMARY & 259385 & $1996 / 04 / 03$ & .00 & .00 & 72.71 & 72.71 \\
\hline FILTER， PRIMRRY & 262379 & $1996 / 08 / 21$ & 21.50 & .00 & 49.71 & 71.21 \\
\hline REPAIR TYPE TOTAL & & & 53.75 & .00 & 186.39 & 240.14 \\
\hline
\end{tabular}


VEHICLE 3228 INT' ETHANOL TANDEM AXLE

\begin{tabular}{|c|c|c|c|c|c|c|}
\hline $\begin{array}{l}\text { REPAIR } \\
\text { CODE } \quad \text { DESCRIPTION }\end{array}$ & $\begin{array}{r}\text { WORK } \\
\text { ORDER }\end{array}$ & $\begin{array}{c}\text { WO } \\
\text { COMPLETED }\end{array}$ & $\begin{array}{l}\text { LABOR } \\
\text { COST }\end{array}$ & $\begin{array}{l}\text { PARTS } \\
\text { COST }\end{array}$ & $\begin{array}{l}\text { OUTSIDE } \\
\text { COST }\end{array}$ & $\begin{array}{l}\text { TOTAL } \\
\text { COST }\end{array}$ \\
\hline REPAIR GROUP TOTAL & & & 107.50 & 13.56 & 10.82 & 131.83 \\
\hline $2300 \mathrm{~L}$ CLUTCH ASSEMBLY & 262379 & $1996 / 08 / 21$ & 21.50 & .00 & .00 & 21.50 \\
\hline REPAIR TYPE TOTAL & & & 21.50 & .00 & .00 & 21.50 \\
\hline REPAIR SUB GROUP TOTAL & & & 21.50 & .00 & .00 & 21.50 \\
\hline REPAIR GROUP TOTAL & & & 21.50 & .00 & .00 & 21.50 \\
\hline $\begin{array}{l}\text { 3107R ALTERNATOR BELT } \\
\text { REPAIR TYPE TOTAL }\end{array}$ & 262379 & $1996 / 08 / 21$ & $\begin{array}{l}21.50 \\
21.50\end{array}$ & $\begin{array}{l}.00 \\
.00\end{array}$ & $\begin{array}{l}15.78 \\
15.78\end{array}$ & $\begin{array}{l}37.28 \\
37.28\end{array}$ \\
\hline REPAIR SUB GROUP TOTAL & & & 21.50 & .00 & 15.78 & 37.28 \\
\hline $3201 \mathrm{R}$ BATTERY & 261019 & $1996 / 06 / 14$ & 43.00 & 41.02 & .00 & 84.02 \\
\hline 3201 I BATTERY & 261846 & $1996 / 07 / 26$ & 118.25 & .00 & .00 & 118.25 \\
\hline REPAIR TYPE TOTAL & & & 161.25 & 41.02 & .00 & 202.27 \\
\hline $3240 R$ STARTER SWITCH & 263344 & $1996 / 09 / 27$ & 21.50 & .00 & .00 & 21.50 \\
\hline REPAIR TYPE TOTAL & & & 21.50 & .00 & .00 & 21.50 \\
\hline REPAIR SUB GROUP TOTAL & & & 182.75 & 41.02 & .00 & 223.77 \\
\hline $3325 R$ GLOW PLUG(S) & 258020 & $1996 / 02 / 16$ & 43.00 & .00 & 25.77 & 68.77 \\
\hline 3325R GLOW PLUG (S) & 258766 & $1996 / 03 / 28$ & 96.75 & .00 & 81.45 & 178.20 \\
\hline 33250 GLOW PLUG(S) & 258766 & $1996 / 03 / 28$ & 86.00 & .00 & .00 & 86.00 \\
\hline REPAIR TYPE TOTAL & & & 225.75 & .00 & 107.22 & 332.97 \\
\hline 3335R IGNITION SWITCH & 263344 & $1996 / 09 / 17$ & .00 & .00 & 14.91 & 14.91 \\
\hline REPAIR TYPE TOTAL & & & .00 & .00 & 14.91 & 14.91 \\
\hline REPAIR SUB GROUP TOTAL & & & 225.75 & .00 & 122.13 & 347.88 \\
\hline $3401 R$ HEADLAMP & 257860 & $1996 / 02 / 06$ & 21.50 & 12.36 & .00 & 33.86 \\
\hline REPAIR TYPE TOTAL & & & 21.50 & 12.36 & .00 & 33.86 \\
\hline 34100 STOPLAMP & 261839 & $1996 / 07 / 19$ & 10.75 & .00 & .00 & 10.75 \\
\hline REPAIR TYPE TOTAL & & & 10.75 & .00 & .00 & 10.75 \\
\hline 3420R TAIL/PARK LAMP & 261839 & $1996 / 07 / 19$ & .00 & 8.67 & .00 & 8.67 \\
\hline REPAIR TYPE TOTAL & & & .00 & 8.67 & .00 & 8.67 \\
\hline 3430R CLEARANCE/MARKER LMP & 251839 & $1996 / 07 / 19$ & .00 & 1.82 & .00 & 1.82 \\
\hline REPAIR TYPE TOTAL & & & .00 & 1.82 & .00 & 1.82 \\
\hline 34330 MISC. LAMPS & 257194 & $1996 / 02 / 06$ & 21.50 & .00 & .00 & 21.50 \\
\hline REPAIR TYPE TOTAL & & & 21.50 & .00 & .00 & 21.50 \\
\hline 34400 STROBE LAMP & 258835 & $1996 / 03 / 11$ & 43.00 & 41.00 & 43.24 & 127.24 \\
\hline REPAIR TYPE TOTAL & & & 43.00 & 41.00 & 43.24 & 127.24 \\
\hline
\end{tabular}



WORK ORDER COST HISTORY

\section{VEHICLE 3228 INT' ETHANOL TANDEM AXLE}

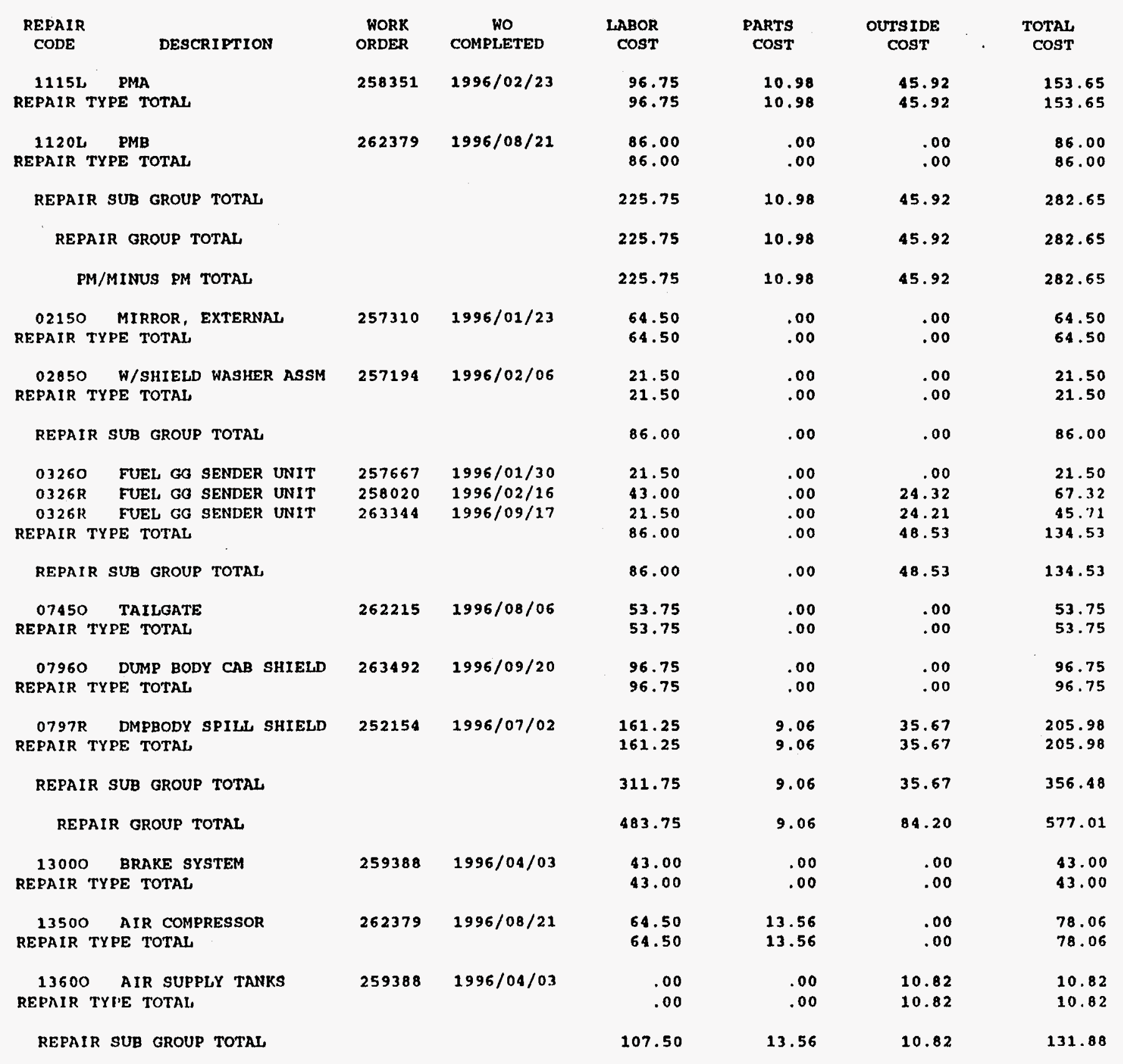


VEHICLE 3228 INT' ETHANOL TANDEM AXLE

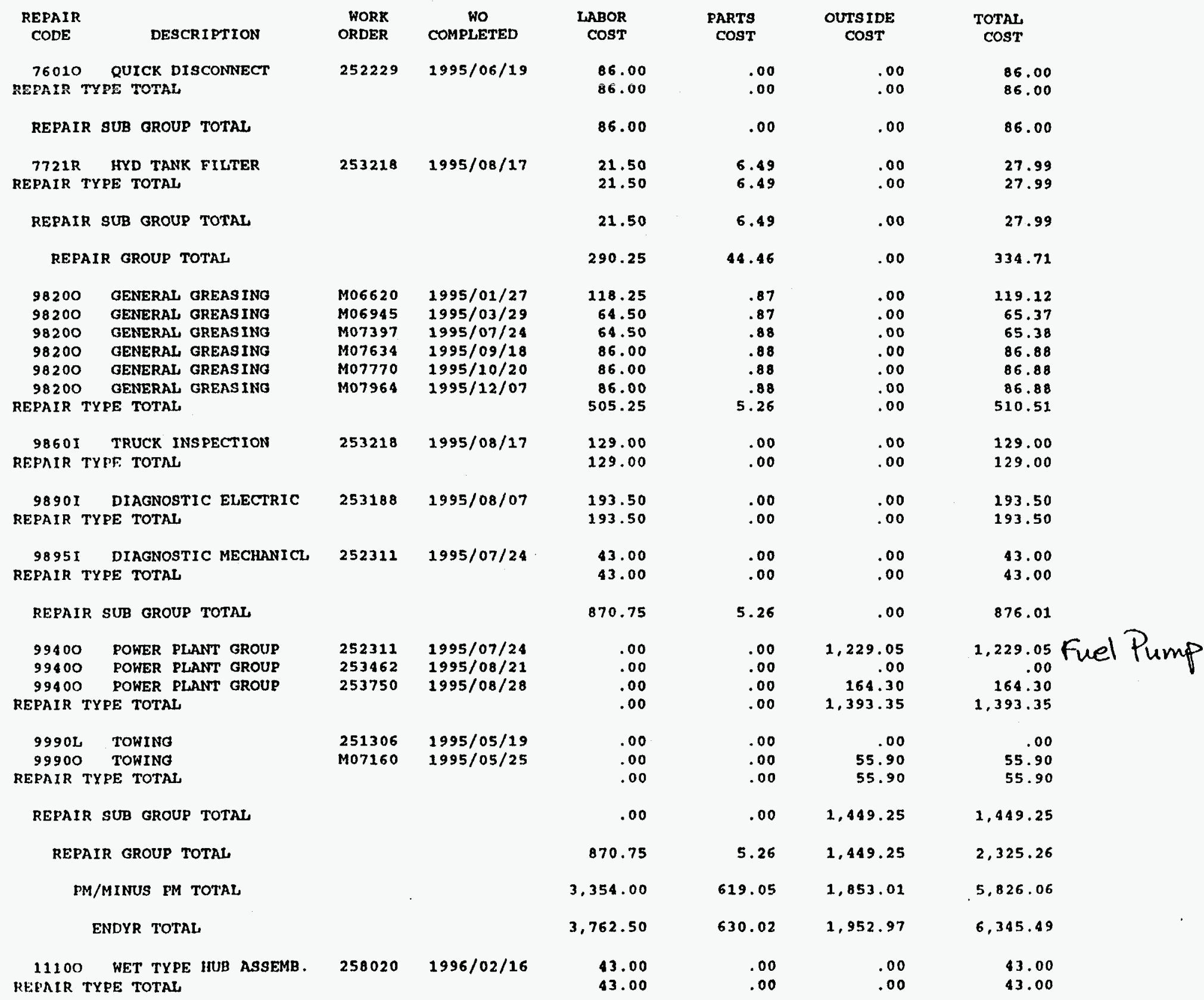


VEHICLE 3228 INT' ETHANOL TANDEM AXLE

\begin{tabular}{|c|c|c|c|c|c|c|}
\hline $\begin{array}{l}\text { REPAIR } \\
\text { CODE }\end{array}$ & $\begin{array}{l}\text { WORK } \\
\text { ORDER }\end{array}$ & $\begin{array}{c}\text { WO } \\
\text { COMPLETED }\end{array}$ & $\begin{array}{l}\text { LABOR } \\
\text { COST }\end{array}$ & $\begin{array}{l}\text { PARTS } \\
\text { COST }\end{array}$ & $\begin{array}{l}\text { OUTSIDE } \\
\text { COST }\end{array}$ & $\begin{array}{l}\text { TOTAL } \\
\text { COST }\end{array}$ \\
\hline 4411R FILTER, SECONDARY & 256349 & $1995 / 12 / 15$ & .00 & .00 & 49.71 & 49.71 \\
\hline REPAIR TYPE TOTAL & & & .00 & .00 & 49.71 & 49.71 \\
\hline REPAIR SUB GROUP TOTAL & & & 86.00 & .00 & 267.84 & 353.84 \\
\hline 45000 ENGINE & 251306 & $1995 / 05 / 19$ & .00 & .00 & .00 & .00 \\
\hline REPAIR TYPE TOTAL & & & .00 & .00 & .00 & .00 \\
\hline REPAIR SUB GROUP TOTAL & & & .00 & .00 & .00 & .00 \\
\hline REPAIR GROUP TOTAL & & & 107.50 & 8.88 & 267.84 & 384.22 \\
\hline 51000 ACCESSORIES, GENERAL & 255142 & $1995 / 10 / 30$ & .00 & .00 & 58.62 & 58.62 \\
\hline REPAIR TYPE TOTAL & & & .00 & .00 & 58.62 & 58.62 \\
\hline 51100 TARPALIN ASSEMBLY & 255142 & $1995 / 10 / 30$ & 247.25 & .84 & .00 & 248.09 \\
\hline 51100 TARPALIN ASSEMBLY & 256244 & $1995 / 12 / 12$ & 86.00 & .00 & .00 & 86.00 \\
\hline REPAIR TYPE TOTAL & & & 333.25 & .84 & .00 & 334.09 \\
\hline REPAIR SUB GROUP TOTAL & & & 333.25 & .84 & 58.62 & 392.71 \\
\hline $52250 \quad 2$-WAY RADIO & 253836 & $1995 / 09 / 01$ & 161.25 & .00 & .00 & 161.25 \\
\hline REPAIR TYPE TOTAL & & & 161.25 & .00 & .00 & 161.25 \\
\hline $\begin{array}{l}\text { 5251R MISC WIRING } \\
\text { REPAIR TYPE TOTAL }\end{array}$ & 253554 & $1995 / 08 / 21$ & $\begin{array}{l}43.00 \\
43.00\end{array}$ & $\begin{array}{l}1.46 \\
1.46\end{array}$ & $\begin{array}{l}.00 \\
.00\end{array}$ & $\begin{array}{l}44.46 \\
44.46\end{array}$ \\
\hline REPAIR SUB GROUP TOTAL & & & 204.25 & 1.46 & .00 & 205.71 \\
\hline 54020 SNOW PLOW BLADE & 249305 & $1995 / 02 / 21$ & 32.25 & .00 & .00 & 32.25 \\
\hline REPATR TYPE TOTAL & & & 32.25 & .00 & .00 & 32.25 \\
\hline REPAIR SUB GROUP TOTAL & & & 32.25 & .00 & .00 & 32.25 \\
\hline REPAIR GROUP TOTAL & & & 569.75 & 2.30 & 58.62 & 630.67 \\
\hline 66000 SP ERUIP ACCESSORIES & 256662 & $1995 / 12 / 28$ & 53.75 & .00 & .00 & 53.75 \\
\hline REPAIR TYPE TOTAL & & & 53.75 & .00 & .00 & 53.75 \\
\hline REPAIR SUB GROUP TOTAL & & & 53.75 & .00 & .00 & 53.75 \\
\hline REPAIR GROUP TOTAL & & & 53.75 & .00 & .00 & 53.75 \\
\hline $7301 R$ HYD LINE, PRESSURE & 248113 & $1995 / 01 / 12$ & 129.00 & 33.65 & .00 & 162.65 \\
\hline REPAIR TYPE TOTAL & & & 129.00 & 33.65 & .00 & 162.65 \\
\hline 73500 HYD LINE, MISC. & 248278 & $1995 / 01 / 23$ & 53.75 & 4.32 & .00 & 58.07 \\
\hline PERAJR TYPE TOTAL & & & 53.75 & 4.32 & .00 & 50.07 \\
\hline REPAIR SUB GROUP TOTAL & & & 182.75 & 37.97 & .00 & 220.72 \\
\hline
\end{tabular}


VEHICLE 3228 INT' ETHANOL TANDEM AXLE

\begin{tabular}{|c|c|c|c|c|c|c|}
\hline $\begin{array}{l}\text { REPAIR } \\
\text { CODE }\end{array}$ & $\begin{array}{l}\text { WORK } \\
\text { ORDER }\end{array}$ & $\begin{array}{c}\text { WO } \\
\text { COMPLETED }\end{array}$ & $\begin{array}{r}\text { LABOR } \\
\text { COST }\end{array}$ & $\begin{array}{r}\text { PARTS } \\
\text { COST }\end{array}$ & $\begin{array}{l}\text { OUTSIDE } \\
\text { COST }\end{array}$ & $\begin{array}{l}\text { TOTAL } \\
\text { COST }\end{array}$ \\
\hline BATTERY & 252960 & $1995 / 07 / 24$ & 139.75 & 164.08 & .00 & 303.83 \\
\hline REPAIP TYPE TOTAL & & & 139.75 & $1 \overline{64} . \overline{0} \overline{0}$ & .00 & 303.83 \\
\hline REPAIR SUB GROUP TOTAL & & & 139.75 & 164.08 & .00 & 303.83 \\
\hline HEADLAMP & 253218 & $1995 / 08 / 17$ & 21.50 & 12.36 & .00 & 33.86 \\
\hline REPAIR TYPE TOTAL & & & 21.50 & 22.36 & .00 & 33.86 \\
\hline 34100 STOPLAMP & 253836 & $1995 / 09 / 01$ & 139.75 & .00 & .00 & 139.75 \\
\hline REPALR TYPE TOTAL & & & 139.75 & .00 & .00 & 139.75 \\
\hline $\begin{array}{l}34150 \text { TURNSIGNAL LAMP } \\
\text { REPAIR TYPE TOTAL }\end{array}$ & 250658 & $1995 / 04 / 18$ & $\begin{array}{l}193.50 \\
193.50\end{array}$ & $\begin{array}{l}7.93 \\
7.93\end{array}$ & $\begin{array}{l}.00 \\
.00\end{array}$ & $\begin{array}{l}201.43 \\
201.43\end{array}$ \\
\hline TAIL/PARK LAMP & 251427 & $1995 / 05 / 12$ & .00 & .00 & 8.30 & 8.30 \\
\hline TAIL/EARK LAMP & 251427 & $1995 / 05 / 12$ & 32.25 & .00 & .00 & 32.25 \\
\hline REPAIR TYPE TOTAL & & & 32.25 & .00 & 8.30 & 40.55 \\
\hline 34300 CLEARANCE/MARKER IMP & 248090 & $1995 / 01 / 12$ & 10.75 & .00 & .00 & 10.75 \\
\hline CLEARANCE/MARKER LMP & 252311 & $1995 / 07 / 24$ & 21.50 & .00 & .00 & 21.50 \\
\hline CLEARANCE/MARKER LMP & 253280 & $1995 / 08 / 07$ & 43.00 & .00 & .00 & 43.00 \\
\hline CLEARANCE/MARKER LMP & 256514 & $1995 / 12 / 19$ & 43.00 & 1.95 & .00 & 44.95 \\
\hline REPAIR TYPE TOTAL & & & 118.25 & 1.95 & .00 & 120.20 \\
\hline 34330 MISC. LAMPS & 248404 & $1995 / 01 / 23$ & 43.00 & .00 & .00 & 43.00 \\
\hline REPAIR TYPE TOTAL & & & 43.00 & .00 & .00 & 43.00 \\
\hline 34400 STROBE LAMP & 248113 & $1995 / 01 / 12$ & 193.50 & .00 & .00 & 193.50 \\
\hline 34400 STROBE LAMP & 253218 & $1995 / 08 / 17$ & 64.50 & .00 & .00 & 64.50 \\
\hline REPAIR TYPE TOTAL & & & 258.00 & .00 & .00 & 258.00 \\
\hline 34500 SANDER/WORK LAMP & 249795 & $1995 / 03 / 10$ & 43.00 & 6.36 & .00 & 49.36 \\
\hline REPAIR TYPE TOTAL & & & 43.00 & 6.36 & .00 & 49.36 \\
\hline 3455R LAMP SWITCH(S) & 253836 & $1995 / 09 / 01$ & .00 & .00 & 13.05 & 13.05 \\
\hline REPAIR TYPE TOTAL, & & & .00 & .00 & 13.05 & 13.05 \\
\hline REPAIR SUB GROUP TOTAL & & & 849.25 & 28.60 & 21.35 & 899.20 \\
\hline REPAIR GROUP TOTAL & & & $1,075.00$ & 192.68 & 21.35 & $1,289.03$ \\
\hline 4212R COOLANT FILTER & 253218 & $1995 / 08 / 17$ & 21.50 & 8.88 & .00 & 30.38 \\
\hline REPAIR TYPE TOTAL & & & 21.50 & 8.86 & .00 & 30.38 \\
\hline REPAIR SUB GROUP TOTAL & & & 21.50 & 8.88 & .00 & 30.38 \\
\hline 4410R FILTER，PRIMARY & 252050 & $1995 / 06 / 12$ & 21.50 & .00 & 145.42 & 166.92 \\
\hline 441 OR FIIIER, PRIMARY & 256349 & $1995 / 12 / 15$ & .00 & .00 & 72.71 & 72.71 \\
\hline 44100 FILTER, PRIMARY & 256349 & $1995 / 12 / 15$ & 64.50 & .00 & .00 & 64.50 \\
\hline REPAIR TYPE TOTAL, & & & 86.00 & .00 & 218.13 & 304.13 \\
\hline
\end{tabular}




\section{VEHICLE 3228 INT ' ETHANOL TANDEM AXLE}

\begin{tabular}{|c|c|c|c|c|c|c|}
\hline $\begin{array}{l}\text { REPAIR } \\
\text { CODE } \quad \text { DESCRIPTION }\end{array}$ & $\begin{array}{r}\text { WORK } \\
\text { ORDER }\end{array}$ & $\begin{array}{l}\text { WO } \\
\text { COMPLETED }\end{array}$ & $\begin{array}{l}\text { LABOR } \\
\text { COST }\end{array}$ & $\begin{array}{r}\text { PARTS } \\
\text { COST }\end{array}$ & $\begin{array}{l}\text { OUTSIDE } \\
\text { COST }\end{array}$ & $\begin{array}{l}\text { TOTAL } \\
\text { COST }\end{array}$ \\
\hline REPAIR SUB GROUP TOTAI, & & & 408.50 & 10.97 & 99.96 & 519.43 \\
\hline REPAIR GROUP TOTAL & & & 408.50 & 10.97 & 99.96 & 519.43 \\
\hline PM/MINUS PM TOTAL & & & 408.50 & 10.97 & 99.96 & 519.43 \\
\hline O2150 MIRROR, EXTERNAL & 252960 & $1995 / 07 / 24$ & 43.00 & .00 & .00 & 43.00 \\
\hline REPAIR TYPE TOTAL & & & 43.00 & .00 & .00 & 43.00 \\
\hline REPAIR SUB GROUP TOTAL & & & 43.00 & .00 & .00 & 43.00 \\
\hline 03250 FUEL GAUGE & 248597 & $1995 / 01 / 30$ & 86.00 & .00 & .00 & 86.00 \\
\hline REPAIR TYPE TOTAL & & & 86.00 & .00 & .00 & 86.00 \\
\hline 0326R FUEL GG SENDER UNIT & 248597 & $1995 / 01 / 30$ & .00 & .00 & 28.90 & 28.90 \\
\hline 0326R FUEL GG SENDER UNIT & 253836 & $1995 / 09 / 01$ & 64.50 & .00 & 27.05 & 91.55 \\
\hline $0326 \mathrm{I}$ FUEL GG SENDER UNIT & 253836 & $1995 / 09 / 01$ & 21.50 & .00 & .00 & 21.50 \\
\hline REPAIR TYPE TOTAL & & & 86.00 & .00 & 55.95 & 141.95 \\
\hline REPAIR SUB GROUP TOTAL & & & 172.00 & .00 & 55.95 & 227.95 \\
\hline REPAIR GROUP TOTAL & & & 215.00 & .00 & 55.95 & 270.95 \\
\hline 13850 SPRING PARKINO BRAKE & 256547 & $1995 / 12 / 19$ & 32.25 & 65.60 & .00 & 97.85 \\
\hline REPAIR TYPE TOTAL & & & 32.25 & 65.60 & .00 & 97.85 \\
\hline REPAIR SUB GROUP TOTAL & & & 32.25 & 65.60 & .00 & 97.85 \\
\hline 1702R TIRE, WORN & 249930 & $1995 / 03 / 14$ & 53.75 & 299.87 & .00 & 353.62 \\
\hline REPAIR TYPE TOTAL & & & 53.75 & 299.87 & .00 & 353.62 \\
\hline REPAIR SUB GROUP TOTAL & & & 53.75 & 299.87 & .00 & 353.62 \\
\hline REPAIR GROUP TOTAL & & & 86.00 & 365.47 & .00 & 451.47 \\
\hline $\begin{array}{l}26000 \text { TRANSMISSION, MANUAL } \\
\text { REPAIR TYPE TOTAL }\end{array}$ & 249305 & $1995 / 02 / 21$ & $\begin{array}{l}86.00 \\
86.00\end{array}$ & $\begin{array}{l}.00 \\
.00\end{array}$ & $\begin{array}{l}.00 \\
.00\end{array}$ & $\begin{array}{l}86.00 \\
86.00\end{array}$ \\
\hline REPAIR SUB GROUP TOTAL & & & 86.00 & .00 & .00 & 86.00 \\
\hline REPAIR GROUP TOTAL & & & 86.00 & .00 & .00 & 86.00 \\
\hline $3100 \mathrm{I}$ CHARGING SYSTEM & 253218 & $1995 / 08 / 17$ & 64.50 & .00 & .00 & 61.50 \\
\hline REPAIR TYPE TOTAL & & & 64.50 & .00 & .00 & 64.50 \\
\hline 31300 ELECTRICAL GROUND & 253218 & $1995 / 08 / 17$ & 21.50 & .00 & .00 & 21.50 \\
\hline REPAIR TYFE TOTNI, & & & 21.50 & .00 & .00 & 21.50 \\
\hline REPAIR SUB GROUP TOTAL & & & 86.00 & .00 & .00 & 86.00 \\
\hline
\end{tabular}




\section{VEHICLE 3228 INT ETHANOL TANDEM AXLE}

\begin{tabular}{|c|c|c|c|c|c|c|}
\hline $\begin{array}{l}\text { REPAIR } \\
\text { CODE }\end{array}$ & $\begin{array}{r}\text { WORK } \\
\text { ORDER }\end{array}$ & $\begin{array}{c}\text { WO } \\
\text { COMPLETED }\end{array}$ & $\begin{array}{l}\text { LABOR } \\
\text { COST }\end{array}$ & $\begin{array}{r}\text { PARTS } \\
\text { COST }\end{array}$ & $\begin{array}{l}\text { OUTSIDE } \\
\text { CosT }\end{array}$ & $\begin{array}{l}\text { TOTAL } \\
\text { COST }\end{array}$ \\
\hline HYD TANK FILTER ASSM & 244659 & $1994 / 08 / 30$ & 86.00 & 1.45 & .00 & 87.45 \\
\hline REPAIR TYPE TOTAL & & & 86.00 & 1.45 & .00 & 87.45 \\
\hline REPAIR SUB GROUP TOTAL & & & 86.00 & 1.45 & .00 & 87.45 \\
\hline REPAIR GROUP TOTAL & & & 236.50 & 1.45 & .00 & 237.95 \\
\hline 98000 MISC INSIDE WORK & $M 05302$ & $1994 / 02 / 25$ & .00 & .00 & 2.56 & 2.56 \\
\hline REPAIR TYPE TOTAL & & & .00 & .00 & 2.56 & 2.56 \\
\hline 98020 STEAMING & 243887 & $1994 / 08 / 01$ & 86.00 & .00 & .00 & 86.00 \\
\hline REQAIR TYPE TOTAL & & & 86.00 & .00 & .00 & 86.00 \\
\hline 98200 GENERAL GREASING & M04995 & $1994 / 01 / 06$ & 124.50 & .80 & .00 & 125.30 \\
\hline 98200 GENERAL GREASING & M05570 & $1994 / 04 / 14$ & 64.50 & .80 & .00 & 65.30 \\
\hline GENERAL GREASINO & M05684 & $1994 / 05 / 18$ & 53.75 & .80 & .00 & 54.55 \\
\hline 98200 GENERAL GREASING & M06071 & $1994 / 09 / 19$ & 86.00 & .80 & 5.12 & 91.92 \\
\hline GENERAL GREAS INO & M06247 & $1994 / 11 / 04$ & 107.50 & .80 & .00 & 108.30 \\
\hline GENERAL GREASING & M06449 & $1994 / 12 / 14$ & 96.75 & .80 & 4.47 & 102.02 \\
\hline REPAIR TYPE TOTAL & & & 533.00 & 4.80 & 9.59 & 547.39 \\
\hline $9860 \mathrm{I}$ TRUCK INSPECTION & 244659 & $1994 / 08 / 30$ & 397.75 & .00 & .00 & 397.75 \\
\hline REPAIR TYPE TOTAL & & & 397.75 & .00 & .00 & 397.75 \\
\hline 98951 DIAGNOSTIC MECHANICL & 244924 & $1994 / 09 / 21$ & 21.50 & .00 & .00 & 21.50 \\
\hline REPAIR TYPE TOTAL & & & 21.50 & .00 & .00 & 21.50 \\
\hline REPAIR SUB GROUP TOTAL & & & 1.038 .25 & 4.80 & 12.15 & 1.055 .20 \\
\hline 9940L POWER PLANT GROUR & 245814 & $1994 / 09 / 30$ & .00 & .00 & .00 & .00 \\
\hline REPAIR TYPE TOTAL & & & .00 & .00 & .00 & .00 \\
\hline REPAIR SUB GROUP TOTAL & & & .00 & .00 & .00 & .00 \\
\hline REPAIR GROUP TOTAL & & & 1.038 .25 & 4.80 & 12.15 & 1.055 .20 \\
\hline PM/MINUS PM TOTAL & & & 6.129 .25 & 369.08 & $1,784.04$ & 8.282 .37 \\
\hline ENDYR TOTAL & & & 6.430 .25 & 397.40 & 1.784 .04 & $8,611.69$ \\
\hline 11100 WET TYPE HUB ASSEMB. & $253 \$ 35$ & $1995 / 08 / 17$ & 86.00 & .00 & .00 & 86.00 \\
\hline 1110R WET TYPE HUB ASSEMB. & 253435 & $1995 / 08 / 17$ & .00 & .00 & 54.04 & 54.04 \\
\hline REPAIR TYPE TOTAL & & & 86.00 & .00 & 54.04 & 140.04 \\
\hline 11151, PMA & 249505 & $1995 / 03 / 01$ & 129.00 & 10.97 & 45.92 & 185.89 \\
\hline REPAIR TYPE TOTAL, & & & 129.00 & 10.97 & 45.92 & 185.89 \\
\hline $11201, \quad$ PMB & 253218 & $1995 / 08 / 17$ & 193.50 & .00 & .00 & 193.50 \\
\hline REPAIR TYPE TOTAL & & & 193.50 & .00 & .00 & 193.50 \\
\hline
\end{tabular}




\begin{tabular}{|c|c|c|c|c|c|c|}
\hline $\begin{array}{l}\text { REPAIR } \\
\text { CODE }\end{array}$ & $\begin{array}{l}\text { WORK } \\
\text { ORDER }\end{array}$ & $\begin{array}{c}\text { พO } \\
\text { COMPLETED }\end{array}$ & $\begin{array}{r}\text { LABOR } \\
\text { COST }\end{array}$ & $\begin{array}{r}\text { PARTS } \\
\text { COST }\end{array}$ & $\begin{array}{l}\text { OUTSIDE } \\
\text { COST }\end{array}$ & $\begin{array}{l}\text { TOTAL } \\
\text { COST }\end{array}$ \\
\hline REPAIR SUB GROUP TOTAL & & & 526.75 & .00 & 416.80 & 943.55 \\
\hline 45000 ENGINE & 239488 & $1994 / 02 / 14$ & 172.00 & .00 & .00 & 172.00 \\
\hline REPAIR TYPE TOTAL & & & 172.00 & .00 & .00 & 172.00 \\
\hline $\begin{array}{l}\text { 45010 BLOCK C CRANKCASE } \\
\text { REPAIR TYPE TOTAL }\end{array}$ & 244192 & $1994 / 08 / 04$ & $\begin{array}{l}311.75 \\
311.75\end{array}$ & $\begin{array}{l}.00 \\
.00\end{array}$ & $\begin{array}{l}.00 \\
.00\end{array}$ & $\begin{array}{l}311.75 \\
311.75\end{array}$ \\
\hline REPAIR SUB GROUP TOTAL & & & 483.75 & .00 & .00 & 483.75 \\
\hline REPAIR GROUP TOTAL & & & $1,354,50$ & 93.16 & 457.50 & $1,905.16$ \\
\hline 51000 ACCESSORIES, OENERAL & 240995 & $1994 / 04 / 12$ & 892.25 & 8.06 & .00 & 900.31 \\
\hline $\begin{array}{l}\text { 5100I ACCESSORIES, GENERAL } \\
\text { REPAIR TYPE TOTAL }\end{array}$ & 240995 & $1994 / 04 / 12$ & $\begin{array}{r}75.25 \\
967.50\end{array}$ & $\begin{array}{r}.00 \\
8.06\end{array}$ & $\begin{array}{l}.00 \\
.00\end{array}$ & $\begin{array}{r}75.25 \\
975.56\end{array}$ \\
\hline $\begin{array}{l}51050 \text { AIR HORN ASSEMBLY } \\
\text { REPAIR TYPE TOTAL }\end{array}$ & 240078 & $1994 / 03 / 01$ & $\begin{array}{l}64.50 \\
64.50\end{array}$ & $\begin{array}{l}.00 \\
.00\end{array}$ & $\begin{array}{l}.00 \\
.00\end{array}$ & $\begin{array}{l}64.50 \\
64.50\end{array}$ \\
\hline TARPALIN ASSEMBLY & 240995 & $1994 / 04 / 12$ & .00 & .00 & 852.72 & 852.72 \\
\hline 51100 TARPALIN ASSEMBLY & 242235 & $1994 / 05 / 26$ & 43.00 & .00 & .00 & 43.00 \\
\hline REINIR TYPE TOTAL & & & 43.00 & .00 & 852.72 & 895.72 \\
\hline W/WASHER BLADES & 239112 & $1994 / 02 / 04$ & 32.25 & .00 & 53.74 & 85.99 \\
\hline REPAIR TYPE TOTAL & & & 32.25 & .00 & 53.74 & 85.99 \\
\hline REPAIR SUB GROUP TOTAL & & & 1.107 .25 & 8.06 & 906.46 & $2,021.77$ \\
\hline $5244 R$ BOX SPRAY PUMP & 243727 & $1994 / 08 / 01$ & 419.25 & 34.43 & 111.91 & 565.59 \\
\hline REPAIR TYPE TOTAL & & & 419.25 & 34.43 & 111.91 & 565.59 \\
\hline REPAIR SUB GROUP TOTAL & & & 419.25 & 34.43 & 111.91 & 565.59 \\
\hline 5407R RUBBING BLOCKS & 239112 & $1994 / 02 / 04$ & 21.50 & .00 & .00 & 21.50 \\
\hline REPAIR TYPE TOTAL & & & 21.50 & .00 & .00 & 21.50 \\
\hline REPAIR SUB GROUP TOTAL & & & 21.50 & .00 & .00 & 21.50 \\
\hline 55500 DUMPBODY LIFT ASSM & 244659 & $1994 / 08 / 30$ & 21.50 & .00 & .00 & 21.50 \\
\hline REPAIR TYPE TOTAL & & & 21.50 & .00 & .00 & 21.50 \\
\hline REPAIR SUB GROUP TOTAL & & & 21.50 & .00 & .00 & 21.50 \\
\hline REPNIR GROUP TOTAL & & & $1,569.50$ & 42.49 & $1,018.37$ & 2.630 .36 \\
\hline 73010 UYD LINE, PRESSURE & 244611 & $1994 / 08 / 23$ & 75.25 & .00 & .00 & 75.25 \\
\hline 73010 HYD LINE, PRESSURE & 246798 & $1994 / 11 / 10$ & 75.25 & .00 & .00 & 75.25 \\
\hline REPAIR TYPE TOTAL & & & 150.50 & .00 & .00 & 150.50 \\
\hline REPAIR SUB GROUP TOTAL & & & 150.50 & .00 & .00 & 150.50 \\
\hline
\end{tabular}


VEHICLE 3228 INT' ETHANOL TANDEM AXLE

\begin{tabular}{|c|c|c|c|c|c|c|}
\hline $\begin{array}{l}\text { REPAIR } \\
\text { CODE }\end{array}$ & $\begin{array}{l}\text { WORK } \\
\text { ORDER }\end{array}$ & $\begin{array}{c}\text { WO } \\
\text { COMPLETED }\end{array}$ & $\begin{array}{r}\text { LABOR } \\
\text { COST }\end{array}$ & $\begin{array}{r}\text { PARTS } \\
\text { COST }\end{array}$ & $\begin{array}{l}\text { OUTS IDE } \\
\text { COST }\end{array}$ & $\begin{array}{l}\text { TOTAL } \\
\text { COST }\end{array}$ \\
\hline CLEARANCE/MARKER LMP & 239303 & $1994 / 02 / 04$ & 43.00 & .00 & .00 & 43.00 \\
\hline CLEARANCE/MARKER LMR & 242235 & $1994 / 05 / 26$ & 32.25 & 10.41 & .00 & 42.66 \\
\hline 34300 CLEARANCE/MARKER LMP & 247325 & $1994 / 12 / 02$ & 64.50 & .00 & .00 & 64.50 \\
\hline REPAIR TYPE TOTAL & & & 139.75 & 10.42 & .00 & 150.16 \\
\hline $\begin{array}{l}34500 \text { SANDER/WORK LAMP } \\
\text { REPAIR TYPE TOTAL }\end{array}$ & 247880 & $1994 / 12 / 21$ & $\begin{array}{l}86.00 \\
86.00\end{array}$ & $\begin{array}{l}.00 \\
.00\end{array}$ & $\begin{array}{l}.00 \\
.00\end{array}$ & $\begin{array}{l}86.00 \\
86.00\end{array}$ \\
\hline REPAIR SUB GROUP TOTAL & & & 537.50 & 30.77 & 13.15 & 581.42 \\
\hline REPAIR GROUP TOTAL & & & 920.00 & 189.05 & 243.30 & $1,352.35$ \\
\hline 4200I COOLING SYSTEM & 246298 & $1994 / 10 / 20$ & 43.00 & .00 & .00 & 43.00 \\
\hline REPAIR TYPE TOTAL & & & 43.00 & .00 & .00 & 43.00 \\
\hline $\begin{array}{l}42040 \text { RAD HOSE, LOWER } \\
\text { REPAIR TYPE TOTAL }\end{array}$ & 240078 & $1994 / 03 / 01$ & $\begin{array}{l}43.00 \\
43.00\end{array}$ & $\begin{array}{l}.00 \\
.00\end{array}$ & $\begin{array}{l}.00 \\
.00\end{array}$ & $\begin{array}{l}43.00 \\
43.00\end{array}$ \\
\hline $\begin{array}{l}\text { 4212R COOLANT FILTER } \\
\text { REPAIR TXPE TOTAL }\end{array}$ & 244659 & $1994 / 08 / 30$ & $\begin{array}{l}161.25 \\
161.25\end{array}$ & $\begin{array}{l}62.44 \\
62.44\end{array}$ & $\begin{array}{l}40.70 \\
40.70\end{array}$ & $\begin{array}{l}264.39 \\
264.39\end{array}$ \\
\hline REPAIR SUB GROUP TOTAL & & & 247.25 & 62.44 & 40.70 & 350.39 \\
\hline 43001 EXHAUST SXSTEM & 243348 & $1994 / 06 / 30$ & 21.50 & .00 & .00 & 21.50 \\
\hline REPAIR TYPE TOTAL & & & 21.50 & .00 & .00 & 21.50 \\
\hline EXHAUST TUBING & 243348 & $1994 / 06 / 30$ & 75.25 & 30.72 & .00 & 105.97 \\
\hline REPAIR TYPE TOTAL & & & 75.25 & 30.72 & .00 & 105.97 \\
\hline REPAIR SUB GROUP TOTAL & & & 96.75 & 30.72 & .00 & 127.47 \\
\hline FUEL SYSTEM & 238856 & $2994 / 01 / 25$ & 118.25 & .00 & .00 & 118.25 \\
\hline$\$ 4000$ FUEL SYSTEM & 240995 & $2994 / 04 / 12$ & 43.00 & .00 & .00 & 43.00 \\
\hline 4400I FUEL SYSTEM & 245814 & $1994 / 09 / 30$ & .00 & .00 & .00 & .00 \\
\hline REPAIR TYPE TOTAL & & & 161.25 & .00 & .00 & 161.25 \\
\hline FILTER， PRIMARY & 238856 & $1994 / 01 / 25$ & 21.50 & .00 & 76.26 & 97.76 \\
\hline FILTER， PRIMARY & 239133 & $1994 / 02 / 04$ & 32.25 & .00 & 145.42 & 177.67 \\
\hline 4410R FILTER, PRIMARY & 243091 & $1994 / 06 / 30$ & 64.50 & .00 & .00 & 64.50 \\
\hline FILTER， PRIMARY & 247793 & $1994 / 12 / 20$ & .00 & .00 & 145.41 & 145,41 \\
\hline FILTER， PRIMARY & 247793 & $1994 / 12 / 20$ & 64.50 & .00 & .00 & 64.50 \\
\hline REPAIR TYPE TOTAL & & & 182.75 & .00 & 367.09 & 549.84 \\
\hline FILTER， SECONDARY & 238856 & $1994 / 01 / 25$ & 21.50 & .00 & .00 & 21.50 \\
\hline FILTER， SECONDARY & 239133 & $1994 / 02 / 04$ & $\$ 3.00$ & .00 & 49.71 & 92.71 \\
\hline REPAIR TYPE TOTAL & & & 64.50 & .00 & 49.71 & 114.21 \\
\hline 44800 ELLECTRONIC CONTROLS & 243091 & $1994 / 06 / 30$ & 118.25 & .00 & .00 & 118.25 \\
\hline REPAIR TYPE TOTAL & & & 118.25 & .00 & .00 & 118.25 \\
\hline
\end{tabular}


VEHICLE 3228 INT' ETHANOL TANDEM AXLE

\begin{tabular}{|c|c|c|c|c|c|c|}
\hline $\begin{array}{l}\text { REPAIR } \\
\text { CODE }\end{array}$ & $\begin{array}{l}\text { WORK } \\
\text { ORDER }\end{array}$ & $\begin{array}{c}\text { WO } \\
\text { COMPLETED }\end{array}$ & $\begin{array}{l}\text { LABOR } \\
\text { COST }\end{array}$ & $\begin{array}{r}\text { PARTS } \\
\text { COSTT }\end{array}$ & $\begin{array}{l}\text { OUTSIDE } \\
\text { COST }\end{array}$ & $\begin{array}{l}\text { TOTAL } \\
\text { COST }\end{array}$ \\
\hline SPRING PARKING BRAKE & 244659 & $1994 / 08 / 30$ & 236.50 & 3.24 & .00 & 239.74 \\
\hline REPAIR TYPE TOTAL & & & 236.50 & 3.24 & .00 & 239.74 \\
\hline REPAIR SUB GROUP TOTAL & & & 376.25 & 13.52 & .00 & 389.77 \\
\hline 18010 & 246718 & $1994 / 11 / 08$ & 139.75 & .00 & 26.66 & 166.41 \\
\hline REPAR TYPE TOTAL & & & 139.75 & .00 & 26.66 & 166.41 \\
\hline REPAIR SUB GROUP TOTAL & & & 139.75 & .00 & 26.66 & 166.41 \\
\hline REPAIR GROUP TOTAL & & & 516.00 & 13.52 & 26.66 & 556.18 \\
\hline 22010 HOUSING, REAR AXLE & 244659 & $1994 / 08 / 30$ & 96.75 & .00 & .00 & 96.75 \\
\hline REPAIR TYPE TOTAL & & & 96.75 & .00 & .00 & 96.75 \\
\hline REPAIR SUB GROUP TOTAL & & & 96.75 & .00 & .00 & 96.75 \\
\hline REPAIR GROUP TOTAL & & & 96.75 & .00 & .00 & 96.75 \\
\hline $3100 \mathrm{I}$ CHARGING SYSTEM & 244408 & $1994 / 08 / 18$ & 43.00 & .00 & .00 & 43.00 \\
\hline REPAIR TYPE TOTAL & & & 43.00 & .00 & .00 & 43.00 \\
\hline ALTERNATOR & 244408 & $1994 / 08 / 18$ & 118.25 & .00 & 230.15 & 348.40 \\
\hline REPAIR TYPE TOTAL & & & 118.25 & .00 & 230.15 & 348.40 \\
\hline REPAIR SUB GROUP TOTAL & & & 161.25 & .00 & 230.15 & 391.40 \\
\hline BATTERY & 238880 & $1994 / 01 / 25$ & 62.25 & 73.98 & .00 & 136.23 \\
\hline BATTERY & 243727 & $1994 / 08 / 01$ & 75.25 & 82.04 & .00 & 157.29 \\
\hline REPAIR TYPE TOTAL & & & 137.50 & 156.02 & .00 & 293.52 \\
\hline 32020 BATTERY CABLE & 238880 & $1994 / 01 / 25$ & 63.00 & 1.68 & .00 & 64.68 \\
\hline & & & & & & \\
\hline BATTERY HOLDOWNS & 238880 & $1994 / 01 / 25$ & 20.75 & .58 & .00 & 21.33 \\
\hline REPAIR TYPE TOTAL & & & 20.75 & .58 & .00 & 21.33 \\
\hline REPAIR SUB GROUP TOTAL & & & 221.25 & 158.28 & .00 & 379.53 \\
\hline 34000 LIGHTING SYSTEM & 243887 & $1994 / 08 / 01$ & 43.00 & .33 & .00 & 43.33 \\
\hline REPAIR TYPE TOTAL & & & 43.00 & .33 & .00 & 43.33 \\
\hline 34010 HEADLAMP & 247167 & $1994 / 11 / 29$ & 21.50 & 10.87 & .00 & 32.37 \\
\hline REPAIR TYPE TOTAL & & & 21.50 & 10.87 & .00 & 32.37 \\
\hline 34100 STOPLAMP & 238259 & $1994 / 01 / 11$ & 96.75 & .00 & 13.15 & 109.90 \\
\hline REIAIR TYPE TOTAI. & & & 96.75 & .00 & 13.15 & 109.90 \\
\hline 34250 BACKUP LAMP & 246726 & $1994 / 11 / 10$ & 150.50 & 9.16 & .00 & 159.66 \\
\hline REPAIR TYPE TOTAL & & & 150.50 & 9.16 & .00 & 259.66 \\
\hline
\end{tabular}


VEHICLE 3228 INT' ETHANOL TANDEM AXLE

\begin{tabular}{|c|c|c|c|c|c|c|}
\hline $\begin{array}{l}\text { REPAIR } \\
\text { CODE }\end{array}$ & $\begin{array}{r}\text { WORR } \\
\text { ORDER }\end{array}$ & $\begin{array}{c}\text { WO } \\
\text { COMPLETED }\end{array}$ & $\begin{array}{r}\text { LABOR } \\
\text { COST }\end{array}$ & $\begin{array}{r}\text { PARTS } \\
\text { COST }\end{array}$ & $\begin{array}{l}\text { OUTSIDE } \\
\text { COST }\end{array}$ & $\begin{array}{l}\text { TOTAL } \\
\text { COST }\end{array}$ \\
\hline REPAIR GROUP TOTAL & & & $3,278.75$ & 350.27 & 297.89 & 4.526 .91 \\
\hline PM/MINUS PM TOTAL & & & $4,106.50$ & 358.85 & $1,156.72$ & $5,622.07$ \\
\hline ENDYR TOTAL & & & $4,106.50$ & 358.85 & $1,156.72$ & $5,622.07$ \\
\hline $1115 \mathrm{~L}$ & 240078 & $1994 / 03 / 01$ & 107.50 & 28.32 & .00 & 135.82 \\
\hline REPAIR TYPE TOTAL & & & 107.50 & 28.32 & .00 & 135.82 \\
\hline $1120 \mathrm{~L} \quad \mathrm{PMB}$ & 244659 & $1994 / 08 / 30$ & 193.50 & .00 & .00 & 193.50 \\
\hline REPAIR TYPE TOTAL & & & 193.50 & .00 & .00 & 193.50 \\
\hline REPAIR SUB GROUP TOTAL & & & 301.00 & 28.32 & .00 & 329.32 \\
\hline REPAIR GROUP TOTAL & & & 301.00 & 28.32 & .00 & 329.32 \\
\hline PM/MINUS PM TOTAL & & & 301.00 & 28.32 & .00 & 329.32 \\
\hline 0150L HEATER ASSEMBLY & 238635 & $1994 / 01 / 24$ & 118.25 & .00 & .00 & 118.25 \\
\hline 0150R HEATER ASSEMBLY & 238880 & $1994 / 01 / 25$ & .00 & .44 & .00 & .44 \\
\hline REPAIR TYPE TOTAL & & & 118.25 & .44 & .00 & 118.69 \\
\hline REPAIR SUB GROUP TOTAL & & & 118.25 & .44 & .00 & 118.69 \\
\hline 0215R MIRROR, EXTERNAL & 243254 & $1994 / 06 / 30$ & 32.25 & 12.98 & .00 & 45.23 \\
\hline REPAIR TYPE TOTAL & & & 32.25 & 12.98 & .00 & 45.23 \\
\hline 0217R MIRROR, EXT CONVEX & 243722 & $1994 / 07 / 18$ & 21.50 & 11.19 & .00 & 32.69 \\
\hline REPAIR TYPE TOTAL & & & 21.50 & 11.19 & .00 & 32.69 \\
\hline REPAIR SUB GROUP TOTAL & & & 53.75 & 24.17 & .00 & 77.92 \\
\hline FUEL GQ SENDER UNIT & 242924 & $1994 / 06 / 21$ & .00 & .00 & 26.06 & 26.06 \\
\hline 03260 FUEL GG SENDER UNIT & 242924 & $1994 / 06 / 21$ & 150.50 & .00 & .00 & 150.50 \\
\hline $0326 \mathrm{I}$ FUEL GO SENDER UNIT & 247167 & $1994 / 11 / 29$ & 43.00 & .00 & .00 & 43.00 \\
\hline REPAIR TYPE TOTAL & & & 193.50 & .00 & 26.06 & 219.56 \\
\hline 03330 LOW AIR PRESS LIGHT & 247093 & $1994 / 11 / 23$ & 32.25 & .00 & .00 & 32.25 \\
\hline REPAIR TYPE TOTAL & & & 32.25 & .00 & .00 & 32.25 \\
\hline REPAIR SUB GROUP TOTAL & & & 225.75 & .00 & 26.06 & 251.81 \\
\hline REPAIR GROUP TOTAL & & & 397.75 & 24.61 & 26.06 & 448.42 \\
\hline BRAKE SYSTEM & 240995 & $1994 / 04 / 12$ & 53.75 & .00 & .00 & 53.75 \\
\hline REPAIR TYPE TOTAL & & & 53.75 & .00 & .00 & 53.75 \\
\hline $13610 \quad$ NIR SUPPLY LINES & 243196 & $1994 / 06 / 24$ & 86.00 & 10.28 & .00 & 96.28 \\
\hline REPAIR TYPE TOTAL & & & 86.00 & 10.28 & .00 & 96.28 \\
\hline
\end{tabular}


VEHICLE 32,28 INT' ETHANOL TANDEM AXLE

\begin{tabular}{|c|c|c|c|c|c|c|}
\hline $\begin{array}{l}\text { REPAIR } \\
\text { CODE }\end{array}$ & $\begin{array}{r}\text { WORK } \\
\text { ORDER }\end{array}$ & $\begin{array}{c}\text { Wo } \\
\text { COMPLETED }\end{array}$ & $\begin{array}{l}\text { LABOR } \\
\text { COST }\end{array}$ & $\begin{array}{l}\text { PARTS } \\
\text { COST }\end{array}$ & $\begin{array}{l}\text { OUTSIDE } \\
\text { COST }\end{array}$ & $\begin{array}{l}\text { TOTAL } \\
\text { COST }\end{array}$ \\
\hline CATALYTIC CONVERTER & 236516 & $1993 / 11 / 08$ & 32.25 & 3.45 & .00 & 35.70 \\
\hline REPAIR TXPE TOTAL & & & 32.25 & 3.45 & .00 & 35.70 \\
\hline REPAIR SUB GROUP TOTAL & & & 32.25 & 3.45 & .00 & 35.70 \\
\hline REPAIR GROUP TOTAL & & & 215.00 & 3.45 & 183.63 & 402.08 \\
\hline 51050 AIR HORN ASSEMBLY & 238090 & $1993 / 12 / 24$ & 43.00 & .00 & 8.83 & 51.83 \\
\hline REPAIR TYPE TOTAL & & & 43,00 & .00 & 8.83 & 51.83 \\
\hline REPAIR SUB GROUP TOTAL & & & 43.00 & .00 & B.83 & 51.83 \\
\hline 52000 ACCESSORY, ELECTRIC & 236707 & $1993 / 11 / 08$ & 43.00 & .00 & .00 & 43.00 \\
\hline REPAIR TYPE TOTAL & & & 43.00 & .00 & .00 & 43.00 \\
\hline REPAIR SUB GROUP TOTAL & & & 43.00 & .00 & .00 & 43.00 \\
\hline 54340 SANDER HXD LINES & 236855 & $1993 / 11 / 17$ & 96.75 & 1.30 & .00 & 98.05 \\
\hline REPAIR TYPE TOTAL & & & 96.75 & 1.30 & .00 & 98.05 \\
\hline REPAIR SUB GROUP TOTAL & & & 96.75 & 1.30 & .00 & 98.05 \\
\hline $5910 \mathrm{R}$ PINTLE HOOK & 231149 & $1993 / 09 / 21$ & .00 & .00 & 45.12 & 45.12 \\
\hline REPATR TYPE TOTAL & & & .00 & .00 & 45.12 & 45.12 \\
\hline REPAIR SUB GROUP TOTAL & & & .00 & .00 & 45.12 & 45,12 \\
\hline REPAIR GROUP TOTAL & & & 182.75 & 1.30 & 53.95 & 238.00 \\
\hline $\begin{array}{l}66000 \text { SP EQUIP ACCESSORIES } \\
\text { REPAIR TYPE TOTAL }\end{array}$ & 237742 & $1993 / 12 / 14$ & $\begin{array}{l}139.75 \\
139.75\end{array}$ & $\begin{array}{l}3.56 \\
3.56\end{array}$ & $\begin{array}{l}.00 \\
.00\end{array}$ & $\begin{array}{l}143.31 \\
143.31\end{array}$ \\
\hline REPAIR SUB GROUP TOTAL & & & 139.75 & 3.56 & .00 & 143.31 \\
\hline REPAIR GROUP TOTAL, & & & 139.75 & 3.56 & .00 & 143.31 \\
\hline 7476L CABLE, CONTL LEVER & 238122 & $1993 / 12 / 28$ & 21.50 & .00 & .00 & 21.50 \\
\hline REPAIR TYPE TOTAL. & & & 21.50 & .00 & .00 & 21.50 \\
\hline REPAIR SUB GROUP TOTAL & & & 21.50 & .00 & .00 & 21.50 \\
\hline REPAIR GROUP TOTAL & & & 21.50 & .00 & .00 & 21.50 \\
\hline NEW VEHICLE PREP. & 231149 & $1993 / 09 / 21$ & .00 & .00 & 212.69 & 212.69 \\
\hline 98300 NEW VEHICLE PREP. & 231149 & $1993 / 09 / 21$ & $3,182.00$ & 350.27 & .00 & $3,532.27$ \\
\hline 98301 NEW VEHICLE PREP. & 231149 & $1993 / 09 / 21$ & .00 & .00 & 271.20 & 271.20 \\
\hline 98300 NEW VEHICLE PREP. & 235741 & $1993 / 10 / 01$ & 96.75 & .00 & 414.00 & 510.75 \\
\hline REPAIR TYPE TOTAL & & & 3.278 .75 & 350.27 & 897.89 & 4.526 .91 \\
\hline REPAIP SUB GROUP TOTAL & & & $3,278.75$ & 350.27 & 897.89 & $4,526.91$ \\
\hline
\end{tabular}


HENNEPIN COUNTY EQUIPMENT MANAGEMENT SYSTEM WORK ORDER COST HISTORY

\section{VEHICLE 3228 INT' ETHLNOL TANDEM AXLE}

\begin{tabular}{|c|c|c|c|c|c|c|}
\hline $\begin{array}{l}\text { REPAIR } \\
\text { CODE }\end{array}$ & $\begin{array}{r}\text { WORK } \\
\text { ORDER }\end{array}$ & $\begin{array}{c}\text { WO } \\
\text { COMPLETED }\end{array}$ & $\begin{array}{r}\text { LABOR } \\
\text { COST }\end{array}$ & $\begin{array}{r}\text { PARTS } \\
\text { COST }\end{array}$ & $\begin{array}{l}\text { OUTSIDE } \\
\text { CosT }\end{array}$ & $\begin{array}{l}\text { TOTAL } \\
\text { COST }\end{array}$ \\
\hline 13500 AIR COMPRESSOR & 262421 & $0000 / 00 / 00$ & .00 & .00 & 9.00 & 9.00 \\
\hline REPAIR TYPE TOTAL & & & .00 & .00 & 9.00 & 9.00 \\
\hline REPAIR SUB GROUP TOTAL & & & .00 & .00 & 9.00 & 9.00 \\
\hline REPAIR GROUP TOTAL & & & .00 & .00 & 9.00 & 9.00 \\
\hline $\begin{array}{l}9895 \mathrm{I} \text { DIAGNOSTIC MECHANICL } \\
\text { REPAIR TYPE TOTAL }\end{array}$ & 262421 & $0000 / 00 / 00$ & 96.75 & .00 & .00 & 96.75 \\
\hline REPAIR SUB GROUP TOTAL & & & 96.75 & .00 & .00 & 96.75 \\
\hline REPAIR GROUP TOTAL & & & 96.75 & .00 & .00 & 96.75 \\
\hline PM/MINUS PM TOTAL & & & 96.75 & .00 & 9.00 & 105.75 \\
\hline ENDYR TOTAL & & & 96.75 & .00 & 9.00 & 105.75 \\
\hline 07380 ENGINE COVER (\$) & 238122 & $1993 / 12 / 28$ & 118.25 & .27 & 21.25 & 139.77 \\
\hline REPAIR TYPE TOTAL & & & 118.25 & .27 & 21.25 & 139.77 \\
\hline 07470 TAILGATE LATCH ASSM & 231149 & $1993 / 09 / 21$ & 75.25 & .00 & .00 & 75.25 \\
\hline REPAIR TYPE TOTAL & & & 75.25 & .00 & .00 & 75.25 \\
\hline REPAIR SUB GROUP TOTAL & & & 193.50 & .27 & 21.25 & 215.02 \\
\hline REPAIR GROUP TOTAL & & & 193.50 & .27 & 21.25 & 215.02 \\
\hline 13610 AIR SUPPLY LINES & 236516 & $1993 / 11 / 08$ & 75.25 & .00 & .00 & 75.25 \\
\hline REPAIR TYPE TOTAL & & & 75.25 & .00 & .00 & 75.25 \\
\hline REPAIR SUB GROUP TOTAL & & & 75.25 & .00 & .00 & 75.25 \\
\hline REPAIR GROUP TOTAL & & & 75.25 & .00 & .00 & 75.25 \\
\hline $\begin{array}{l}\text { 42040 RAD HOSE, LOWER } \\
\text { REPAIR TYPE TOTAL }\end{array}$ & 238122 & $1993 / 12 / 28$ & $\begin{array}{l}21.50 \\
21.50\end{array}$ & .00 & .00 & 21.50 \\
\hline REPAIR SUB GROUP TOTAL & & & 21.50 & .00 & .00 & 21.50 \\
\hline 4410R FILTER, PRIMARY & 237390 & $1993 / 12 / 03$ & 10.75 & .00 & 183.63 & 194.38 \\
\hline REPAIR TYPE TOTAL & & & 10.75 & .00 & 183.63 & 194.38 \\
\hline 4411R FILTER, SECONDARY & 237390 & $1993 / 12 / 03$ & 10.75 & .00 & .00 & 10.75 \\
\hline REPAIR TYPE TOTAL & & & 10.75 & .00 & .00 & 10.75 \\
\hline 44200 FUEL PUMP & 236855 & $1993 / 11 / 17$ & 139.75 & .00 & .00 & 139.75 \\
\hline RFPAR TYPE TOTAL & & & 139.75 & .00 & .00 & 139.75 \\
\hline REPAIR SUB GROUP TOTAL, & & & 161.25 & .00 & 183.63 & 344.88 \\
\hline
\end{tabular}


VEHICLE 3221 INT' ETHANOL TANDEM AXLE

\begin{tabular}{|c|c|c|c|c|c|c|}
\hline $\begin{array}{l}\text { REPAIR } \\
\text { CODE }\end{array}$ & $\begin{array}{r}\text { WORK } \\
\text { ORDER }\end{array}$ & $\begin{array}{c}\text { WO } \\
\text { COMPLETED }\end{array}$ & $\begin{array}{r}\text { LABOR } \\
\text { COST }\end{array}$ & $\begin{array}{r}\text { PARTS } \\
\text { COST }\end{array}$ & $\begin{array}{l}\text { OUTS IDE } \\
\text { COST }\end{array}$ & $\begin{array}{r}\text { TOTAL } \\
\text { COST }\end{array}$ \\
\hline DIAGNOSTIC ELECTRIC & 256804 & $1996 / 02 / 23$ & 64.50 & .00 & .00 & 64.50 \\
\hline DIAGNOSTIC ELECTRIC & 263390 & $1996 / 09 / 17$ & 32.25 & .00 & .00 & 32.25 \\
\hline REPAIR TYPE TOTAL & & & 96.75 & .00 & .00 & 96.75 \\
\hline 98951 DIAGNOSTIC MECHANICL & 260537 & $1996 / 05 / 17$ & 43.00 & .00 & .00 & 43.00 \\
\hline REPAIR TYPE TOTAL & & & 43.00 & .00 & .00 & 43.00 \\
\hline REPAIR SUB GROUP TOTAL & & & 677.25 & 5.28 & 19.68 & 702.21 \\
\hline CHASSIS GROUP & 263556 & $1996 / 09 / 26$ & .00 & .00 & 197.60 & 197.60 \\
\hline REPAIR TYPE TOTAL & & & .00 & .00 & 197.60 & 197.60 \\
\hline REPALR SUB GROUP TOTAL & & & .00 & .00 & 197.60 & 197.60 \\
\hline REPAIR GROUP TOTAL & & & 677.25 & 5.28 & 217.28 & 899.81 \\
\hline PM/MINUS PM TOTAL & & & $5,031.00$ & 1.222 .70 & $1,082.19$ & $7,335.89$ \\
\hline ENDYR TOTAI, & & & $5,203.00$ & 1.237 .28 & 1.128 .11 & 7.568 .39 \\
\hline UNIT TOTAL & & & $22,631.78$ & $2,316.70$ & $5,464.33$ & 30.412 .81 \\
\hline
\end{tabular}


VEHICLE 3221 INT' ETHANOL TANDEM AXLE

\begin{tabular}{|c|c|c|c|c|c|c|}
\hline $\begin{array}{l}\text { REPAIR } \\
\text { CODE }\end{array}$ & $\begin{array}{r}\text { WORK } \\
\text { ORDER }\end{array}$ & $\begin{array}{c}\text { WO } \\
\text { COMPLETED }\end{array}$ & $\begin{array}{r}\text { LABOR } \\
\text { COST }\end{array}$ & $\begin{array}{r}\text { PARTS } \\
\text { COST }\end{array}$ & $\begin{array}{l}\text { OUTSIDE } \\
\text { COST }\end{array}$ & $\begin{array}{l}\text { TOTAL } \\
\text { COST }\end{array}$ \\
\hline TARPALIN ASSEMBLY & 259063 & $1996 / 03 / 20$ & 43.00 & .00 & .00 & 43.00 \\
\hline 51100 TARPALIN ASSEMBLY & 263390 & $1996 / 09 / 17$ & 43.00 & .00 & .00 & 43.00 \\
\hline REPAIR TYPE TOTAL & & & 268.75 & .98 & .00 & 269.73 \\
\hline REPAIR SUB GROUP TOTAL & & & 419.25 & .98 & 153.24 & 573.47 \\
\hline 52250 2-WAY RADIO & 262270 & $1996 / 08 / 08$ & 21.50 & .00 & .00 & 21.50 \\
\hline REPAIR TYPE TOTAL & & & 21.50 & .00 & .00 & 21.50 \\
\hline 52400 BOX SHAKER & 261425 & $1996 / 07 / 02$ & 86.00 & .00 & .00 & 86.00 \\
\hline REPAIR TYPE TOTAL & & & 86.00 & .00 & .00 & 86.00 \\
\hline REPAIR SUB GROUP TOTAL & & & 107.50 & .00 & .00 & 107.50 \\
\hline REPAIR GROUP TOTAL & & & 526.75 & .98 & 153.24 & 680.97 \\
\hline 66000 SP EQUIP ACCESSORIES & 261972 & $1996 / 07 / 26$ & 43.00 & .00 & .00 & 43.00 \\
\hline 66000 SP EQUIP ACCESSORIES & 263556 & $1996 / 09 / 26$ & 21.50 & .00 & .00 & 21.50 \\
\hline REPAIR TYPE TOTAL & & & 64.50 & .00 & .00 & 64.50 \\
\hline REPAIR SUB GROUP TOTAL & & & 64.50 & .00 & .00 & 64.50 \\
\hline REPAIR GROUP TOTAL & & & 64.50 & .00 & .00 & 64.50 \\
\hline $71100 \quad$ HYD PUMP & 259528 & $1996 / 05 / 02$ & .00 & .00 & 274.88 & 274.88 \\
\hline $7110 R$ IXYD PUMP & 259528 & $1996 / 05 / 02$ & 236.50 & .00 & .00 & 236.50 \\
\hline 71101 HYD PUMP & 259528 & $1996 / 05 / 02$ & 21.50 & .00 & .00 & 21.50 \\
\hline REPAIR TYPE TOTAL & & & 258.00 & .00 & 274.88 & 532.88 \\
\hline REPAIR SUB GROUP TOTAL & & & 258.00 & .00 & 274.88 & 532.88 \\
\hline 7721R HYD TANK FILTER & 262381 & $1996 / 09 / 03$ & 21.50 & 6.49 & .00 & 27.99 \\
\hline REPAIR TYPE TOTAL & & & 21.50 & 6.49 & .00 & 27.99 \\
\hline REPAIR SUB GROUP TOTAL & & & 21.50 & 6.49 & .00 & 27.99 \\
\hline REPAIR GROUP TOTAL & & & 279.50 & 6.49 & $274.8 B$ & 560.87 \\
\hline 98200 GENERAL GREASING & M08136 & $1996 / 01 / 25$ & 86.00 & .88 & .00 & 86.88 \\
\hline 98200 GENERAL GREASING & M08222 & $1996 / 02 / 07$ & 86.00 & .88 & .00 & 86.88 \\
\hline 98200 GENERAL GREAS ING & $M 08432$ & $1996 / 03 / 15$ & 86.00 & .88 & 9.84 & 96.72 \\
\hline GENERAL GREASING & M08484 & $1996 / 04 / 03$ & 86.00 & .88 & .00 & 86.88 \\
\hline 98200 GENERAL GREASINO & MOB720 & $1996 / 05 / 23$ & 86.00 & $.8 B$ & 9.84 & 96.72 \\
\hline $9 B 200$ GENERAL GREASING & M09046 & $1996 / 08 / 15$ & 86.00 & .88 & .00 & 86.88 \\
\hline REPAIR TYPE TOTAL & & & 516.00 & 5.28 & 19.68 & 540.96 \\
\hline 98601 TRUCK INSPECTION & 262381 & $1996 / 09 / 03$ & 21.50 & .00 & .00 & 21.50 \\
\hline REPAIR TYPE TOTAL & & & 21.50 & .00 & .00 & 21.50 \\
\hline
\end{tabular}


VEHICLE 3221 INT' ETHANOL TANDEM AXLE

\begin{tabular}{|c|c|c|c|c|c|c|}
\hline $\begin{array}{l}\text { REPAIR } \\
\text { CODE }\end{array}$ & $\begin{array}{l}\text { WORK } \\
\text { ORDER }\end{array}$ & $\begin{array}{c}\text { WO } \\
\text { COMPLETED }\end{array}$ & $\begin{array}{r}\text { LAABOR } \\
\text { COST }\end{array}$ & $\begin{array}{l}\text { PARTS } \\
\text { COST }\end{array}$ & $\begin{array}{l}\text { OUTSIDE } \\
\text { CosT }\end{array}$ & $\begin{array}{l}\text { TOTAL } \\
\text { COST }\end{array}$ \\
\hline EXHAUST TUBING & 262164 & $1996 / 07 / 31$ & 21.50 & .00 & $=00$ & 21.50 \\
\hline REPAIR TYPE TOTAL & & & 21.50 & .00 & .00 & 21.50 \\
\hline 4350R CLAMPS & 262164 & $1996 / 07 / 31$ & 10.75 & 15.36 & .00 & 26.11 \\
\hline REPAIR TYPE TOTAL & & & 10.75 & 15.36 & .00 & 26.11 \\
\hline REPAIR SUB GROUP TOTAL & & & 86.00 & 15.36 & 8.86 & 110.22 \\
\hline FILTER， PRIMARY & 257539 & $1996 / 01 / 26$ & 64.50 & .00 & 63.97 & 128.47 \\
\hline FILTER， PRIMARY & 259063 & $1996 / 03 / 20$ & 53.75 & .00 & 72.71 & 126.46 \\
\hline 4410R FILTER， PRIMARY & 261897 & $1996 / 07 / 23$ & 32.25 & .00 & .00 & 32.25 \\
\hline REPAIR TYPE TOTAL & & & 150.50 & .00 & 136.68 & 287.18 \\
\hline 4411R FILTER， SECONDARY & 259063 & $1996 / 03 / 20$ & .00 & .00 & 46.92 & 46.92 \\
\hline 4411R FILTER, SECONDARY & 261897 & $1996 / 07 / 23$ & 32.25 & .00 & .00 & 32.25 \\
\hline REPAIR TYPE TOTAL & & & 32.25 & .00 & 46.92 & 79.17 \\
\hline REPAIR SUB GROUP TOTAL & & & 182.75 & .00 & 183.60 & 366.35 \\
\hline ENGINE & 259085 & $1996 / 03 / 20$ & 43.00 & .00 & .00 & 43.00 \\
\hline ENGINE & 259528 & $1996 / 05 / 02$ & 75.25 & .00 & .00 & 75.25 \\
\hline ENGINE & 263316 & $1996 / 09 / 12$ & 43.00 & .00 & .00 & 43.00 \\
\hline REPAIR TYFE TOTNL & & & 161.25 & .00 & .00 & 161.25 \\
\hline 45700 VENTILATING SYSTEM & 263316 & $1996 / 09 / 12$ & 43.00 & 3.37 & .00 & 46.37 \\
\hline REPAIR TYPE TOTAL & & & 43.00 & 3.37 & .00 & 46.37 \\
\hline $\begin{array}{l}45750 \text { VALVE COVER GASKET } \\
\text { REPAIR TYPE TOTAL }\end{array}$ & 262270 & $1996 / 08 / 08$ & $\begin{array}{l}43.00 \\
43.00\end{array}$ & $\begin{array}{l}.00 \\
.00\end{array}$ & $\begin{array}{l}.00 \\
.00\end{array}$ & $\begin{array}{l}43.00 \\
43.00\end{array}$ \\
\hline 4584R SEALS, MISC. & 257664 & $1996 / 02 / 29$ & .00 & .00 & 26.71 & 26.71 \\
\hline REPAIR TYPE TOTAL & & & .00 & .00 & 26.71 & 26.71 \\
\hline REPAIR SUB GROUP TOTAL & & & 247.25 & 3.37 & 26.71 & 277.33 \\
\hline 4632R PCV VALVE & 257539 & $1996 / 01 / 26$ & 204.25 & 2.12 & 24.37 & 230.74 \\
\hline REPAIR TYPE TOTAL & & & 204.25 & 2.12 & 24.37 & 230.74 \\
\hline REPAIR SUB GROUP TOTAL & & & 204.25 & 2.12 & 24.37 & 230.74 \\
\hline REPAIR GROUP TOTAL & & & 924.50 & 29.73 & 243.54 & 1.197 .77 \\
\hline ACCESSORIES, GENERAL & 258435 & $1996 / 02 / 28$ & 21.50 & .00 & .00 & 21.50 \\
\hline REPAIR TYPE TOTAL & & & 21.50 & .00 & .00 & 21.50 \\
\hline $5102 R$ MUD FLAP (S) & 261972 & $1996 / 07 / 26$ & 129.00 & .00 & 153.24 & 282.24 \\
\hline REPAIR TYPE TOTAL & & & 129.00 & .00 & 153.24 & 282.24 \\
\hline 51100 TARPALIN ASSEMBLY & 259045 & $1996 / 03 / 18$ & 182.75 & .96 & .00 & 183.73 \\
\hline
\end{tabular}




\section{VEHICLE 3221 INT' ETHANOL TANDEM AXLE}

\begin{tabular}{|c|c|c|c|c|c|c|}
\hline $\begin{array}{l}\text { REPAIR } \\
\text { CODE }\end{array}$ & $\begin{array}{l}\text { WORK } \\
\text { ORDER }\end{array}$ & $\begin{array}{c}\text { WO } \\
\text { COMPLETED }\end{array}$ & $\begin{array}{l}\text { LABOR } \\
\text { COST }\end{array}$ & $\begin{array}{r}\text { PARTS } \\
\text { COST }\end{array}$ & $\begin{array}{l}\text { OUTSIDE } \\
\text { COST }\end{array}$ & $\begin{array}{l}\text { TOTAL } \\
\text { COST }\end{array}$ \\
\hline REPAIR SUB GROUP TOTAL & & & 193.50 & .00 & 18.89 & 212.39 \\
\hline GLOW PLUG(S) & 257664 & $1996 / 02 / 29$ & 75.25 & .00 & 25.77 & 101.02 \\
\hline GLOW PLUA(s) & 258677 & $1996 / 03 / 28$ & 43.00 & .00 & .00 & 43.00 \\
\hline GLOW PLUG(s) & 262381 & $1996 / 09 / 03$ & .00 & .00 & 25.77 & 25.77 \\
\hline 3325R GLOW PLUG(S) & 263390 & $1996 / 09 / 17$ & 21.50 & .00 & 25.77 & 47.27 \\
\hline REPAIR TYPE TOTAL & & & 139.75 & .00 & 77.31 & 217.06 \\
\hline REPAIR SUB GROUP TOTAI & & & 139.75 & .00 & 77.31 & 217.06 \\
\hline 3415R TURNSIGNAL LAMP & 259528 & $1996 / 05 / 02$ & .00 & .00 & 22.55 & 22.55 \\
\hline REPAIR TYPE TOTAL & & & .00 & .00 & 22.55 & 22.55 \\
\hline TAIL/PARK LLAMP & 257446 & $1996 / 01 / 23$ & 75.25 & .00 & .00 & 75.25 \\
\hline 34200 TAIL/PARK LLAMP & 259528 & $1996 / 05 / 02$ & 53.75 & 1.34 & .00 & 55.09 \\
\hline REPAIR TYPE TOTAL & & & 129.00 & 1.34 & .00 & 130.34 \\
\hline CLEARANCE/MARKER LMP & 257664 & $1996 / 02 / 29$ & 43.00 & .00 & .00 & 43.00 \\
\hline CLEARANCE/MARKER LMP & 258695 & $1996 / 03 / 05$ & 21.50 & 1.83 & .00 & 23.33 \\
\hline CLEARANCE/MARKER LMP & 259528 & $1996 / 05 / 02$ & 86.00 & .00 & .00 & 86.00 \\
\hline REPAIR TYPE TOTAL & & & 150.50 & 1.83 & .00 & 152.33 \\
\hline SPOTLIGHT LAMP & 257539 & $1996 / 01 / 26$ & 64.50 & .00 & .00 & 64.50 \\
\hline REPAIR TYPE TOTAL, & & & 64.50 & .00 & .00 & 64.50 \\
\hline REPAIR SUB GROUP TOTAL & & & 344.00 & 3.17 & 22.55 & 369.72 \\
\hline REPAIR GROUP TOTAL & & & 677.25 & 3.17 & 118.75 & 799.17 \\
\hline$\$ 1300$ SUPERCHARGER & 257654 & $1996 / 02 / 29$ & 75.25 & .00 & .00 & 75.25 \\
\hline SUPERCHARGER & 258677 & $1996 / 03 / 28$ & 43.00 & .00 & .00 & 43.00 \\
\hline REPAIR TYPE TOTAL & & & 118.25 & .00 & .00 & 118.25 \\
\hline REPAIR SUB GROUP TOTAL & & & 118.25 & .00 & .00 & 118.25 \\
\hline $\begin{array}{l}\text { 4204L RAD HOSE, LOWER } \\
\text { REPAIR TYPE TOTAL }\end{array}$ & 257342 & $1996 / 01 / 23$ & $\begin{array}{l}43.00 \\
43.00\end{array}$ & $\begin{array}{l}.00 \\
.00\end{array}$ & $\begin{array}{l}.00 \\
.00\end{array}$ & $\begin{array}{l}43.00 \\
43.00\end{array}$ \\
\hline 42110 ANTIFREEZE & 263316 & $1996 / 09 / 12$ & 21.50 & .00 & .00 & 21.50 \\
\hline REPAIR TYPE TOTAL & & & 21.50 & .00 & .00 & 21.50 \\
\hline 4212R COOLANT FILTER & 262381 & $1996 / 09 / 03$ & 21.50 & 8.88 & .00 & 30.38 \\
\hline REPAIR TYPE TOTAL & & & 21.50 & 8.88 & .00 & 30.38 \\
\hline REPAIR SUB GROUP TOTAL & & & B6.00 & B. 88 & .00 & 94.88 \\
\hline 4302R EXT MANIFOLD GASKET & 258677 & $1996 / 03 / 28$ & 53.75 & .00 & 8.86 & 62.61 \\
\hline REPAIR TYPE TOTAI, & & & 53.75 & .00 & 8.86 & 62.61 \\
\hline
\end{tabular}


VEHICLE 3221 INT' ETHANOL TANDEM AXLE

\begin{tabular}{|c|c|c|c|c|c|c|}
\hline $\begin{array}{l}\text { REPAIR } \\
\text { CODE }\end{array}$ & $\begin{array}{r}\text { WORK } \\
\text { ORDER }\end{array}$ & $\begin{array}{c}\text { WO } \\
\text { COMPLETED }\end{array}$ & $\begin{array}{r}\text { LABOR } \\
\text { COST }\end{array}$ & $\begin{array}{r}\text { PARTS } \\
\cos T\end{array}$ & $\begin{array}{c}\text { OUTSIDE } \\
\text { CosT }\end{array}$ & $\begin{array}{l}\text { TOTAL } \\
\text { COST }\end{array}$ \\
\hline 13000 BRAKE SYSTEM & 259231 & $1996 / 04 / 03$ & 86.00 & .00 & .00 & 86.00 \\
\hline REPAIR TYPE TOTAL & & & 86.00 & .00 & .00 & 86.00 \\
\hline 13500 AIR COMPRESSOR & 257664 & $1996 / 02 / 29$ & 75.25 & 13.56 & 9.00 & 97.81 \\
\hline REPAIR TYPE TOTAL & & & 75.25 & 13.56 & 9.00 & 97.81 \\
\hline 13550 AIR DRYER ASSEMBLY & 257664 & $1996 / 02 / 29$ & 43.00 & .00 & 11.28 & 54.28 \\
\hline REPAIR TYPE TOTAL & & & 43.00 & .00 & 11.28 & 54.28 \\
\hline SPRING PARKING BRARE & 258435 & $1996 / 02 / 28$ & 43.00 & .00 & .00 & 43.00 \\
\hline 1385R SPRING PARKING BRAKE & 260785 & $1996 / 05 / 31$ & 107.50 & 65.60 & .00 & 173.10 \\
\hline 1385R SPRING PARKING BRAKE & 262381 & $1996 / 09 / 03$ & 64.50 & 131.20 & .00 & 195.70 \\
\hline REPAIR TYPE TOTAL & & & 215.00 & 196.80 & .00 & 411.80 \\
\hline REPAIR SUB GROUP TOTAL & & & 419.25 & 210.36 & 20.28 & 649.89 \\
\hline 1670R REAR SPRING ASSEMBLY & 263556 & $1996 / 09 / 26$ & 462.25 & .00 & .00 & 462.25 \\
\hline REPAIR TYPE TOTAL & & & 462.25 & .00 & .00 & 462.25 \\
\hline REPAIR SUB GROUP TOTAL & & & 462.25 & .00 & .00 & 462.25 \\
\hline 1702R TIRE，WORN & 263556 & $1996 / 09 / 26$ & 129.00 & 923.96 & .00 & $1,052.96$ \\
\hline REPAIR TYPE TOTAL & & & 129.00 & 923.96 & .00 & $1,052.96$ \\
\hline REPAIR SUB GROUP TOTAL & & & 129.00 & 923.96 & .00 & 1.052 .96 \\
\hline REPAIR GROUP TOTAL & & & $1,010.50$ & $1,134.32$ & 20.28 & $2,165.10$ \\
\hline $2300 \mathrm{~L}$ CLUTCH ASSEMBLY & 262381 & $1996 / 09 / 03$ & 21.50 & .00 & .00 & 21.50 \\
\hline REPAIR TYPE TOTAL & & & 21.50 & .00 & .00 & 21.50 \\
\hline & 257664 & $1996 / 02 / 29$ & 43.00 & .00 & .00 & 43.00 \\
\hline REPAIR TYPE TOTAL & & & 43.00 & .00 & .00 & 93.00 \\
\hline REPAIR SUB GROUP TOTAL & & & 64.50 & .00 & .00 & 64.50 \\
\hline 26000 TRANSMISSION, MANUAL & 257664 & $1996 / 02 / 29$ & 64.50 & .00 & 6.39 & 70.89 \\
\hline REPAIR TYPE TOTAL & & & 64.50 & .00 & 6.39 & 70.89 \\
\hline 26020 TRANS REAR HOUSING & 257664 & $1996 / 02 / 29$ & 161.25 & .00 & .00 & 161.25 \\
\hline REPAIR TYPE TOTAL & & & 161.25 & .00 & .00 & 161.25 \\
\hline REPAIR SUB GROUP TOTAL & & & 225.75 & .00 & 6.39 & 232.14 \\
\hline REPAIR GROUP TOTAL & & & 290.25 & .00 & 6.39 & 296.64 \\
\hline 3107R ALTERNATOR BELT & 259085 & $1996 / 03 / 20$ & 129.00 & .00 & 18.89 & 147.89 \\
\hline 3107I, ALTERNATOR BELT & 258677 & $1996 / 03 / 28$ & 64.50 & .00 & .00 & 64.50 \\
\hline REPAIR TYLE TOTAL & & & 193.50 & .00 & 18.89 & 212.39 \\
\hline
\end{tabular}


VEHICLE 3221 INT' ETHANOL TANDEM AXLE

\begin{tabular}{|c|c|c|c|c|c|c|}
\hline $\begin{array}{l}\text { REPAIR } \\
\text { CODE } \quad \text { DESCRIPTION }\end{array}$ & $\begin{array}{l}\text { WORK } \\
\text { ORDER }\end{array}$ & $\begin{array}{c}\text { WO } \\
\text { COMPLETED }\end{array}$ & $\begin{array}{r}\text { LABOR } \\
\text { COST }\end{array}$ & $\begin{array}{l}\text { PARTS } \\
\text { COST }\end{array}$ & $\begin{array}{l}\text { OUTSIDE } \\
\text { COST }\end{array}$ & $\begin{array}{l}\text { TOTAL } \\
\text { COST }\end{array}$ \\
\hline ENDYR TOTAL, & & & $5,349.63$ & 442.19 & 776.60 & $6,568.42$ \\
\hline $1115 \mathrm{~L}$ PMA & 258695 & $1996 / 03 / 05$ & .00 & 3.60 & .00 & 3.60 \\
\hline 1115L PMA & 263556 & $1996 / 09 / 26$ & 129.00 & 10.98 & 45.92 & 185.90 \\
\hline REPAIR TYPE TOTAL & & & 129.00 & 14.58 & 45.92 & 189.50 \\
\hline $1120 \mathrm{~L}$ PMB & 262381 & $1996 / 09 / 03$ & 43.00 & .00 & .00 & 43.00 \\
\hline REPAIR TYPE TOTAL & & & 43.00 & .00 & .00 & 43.00 \\
\hline REPAIR SUB GROUP TOTAL & & & 172.00 & 14.58 & 45.92 & 232.50 \\
\hline REPAIR GROUP TOTAL & & & 172.00 & 14.58 & 45.92 & 232.50 \\
\hline PM/MINUS PM TOTAL & & & 172.00 & 14.58 & 45.92 & 232.50 \\
\hline 0215R MIRROR, EXTERNAL & 256804 & $1996 / 02 / 23$ & 21.50 & .00 & .00 & 21.50 \\
\hline 02150 MIRROR, EXTERNAL & 256804 & $1996 / 02 / 23$ & .00 & 13.81 & .00 & 13.81 \\
\hline 0215R MIRROR，EXTERNAL & 257664 & $1996 / 02 / 29$ & 43.00 & 13.81 & .00 & 56.81 \\
\hline 0215R MIRROR， EXTERNAL & 262501 & $1996 / 08 / 15$ & 43.00 & 15.11 & .00 & 58.11 \\
\hline REPAIR TYPE TOTAL & & & 107.50 & 42.73 & .00 & 150.23 \\
\hline REPAIR SUB GROUP TOTAL & & & 107.50 & 42.73 & .00 & 150.23 \\
\hline 03000 GAUGES/INSTRUMENTS & 259989 & $1996 / 04 / 29$ & 107.50 & .00 & .00 & 107.50 \\
\hline REPAIR TYPE TOTAL & & & 107.50 & .00 & .00 & 107.50 \\
\hline FUEL GG SENDER UNIT & 261109 & $1996 / 06 / 14$ & 21.50 & .00 & 26.82 & 48.32 \\
\hline 0326 I FUEL GG SENDER UNIT & 261109 & $1996 / 06 / 14$ & 21.50 & .00 & .00 & 21.50 \\
\hline REPAIR TXPE TOTAL & & & 43.00 & .00 & 26.82 & 69.82 \\
\hline 03401 SPEEDOMETER & 261109 & $1996 / 06 / 14$ & $\mathbf{4 3 . 0 0}$ & .00 & .00 & 43.00 \\
\hline REPAIR TYPE TOTAL & & & 43.00 & .00 & .00 & 43.00 \\
\hline 0344R SPEDOMETR SENDR UNIT & 261109 & $1996 / 06 / 14$ & 43.00 & .00 & 21.01 & 64.01 \\
\hline REPAIR TYPE TOTAL & & & 43.00 & .00 & 21.01 & 64.01 \\
\hline REPAIR SUB GROUP TOTAL & & & 236.50 & .00 & 47.83 & 284.33 \\
\hline 07950 DUMP BODY & 258950 & $1996 / 03 / 14$ & 43.00 & .00 & .00 & 43.00 \\
\hline REPAIR TYPE TOTAL & & & 43.00 & .00 & .00 & 43.00 \\
\hline 07970 DMPBODY SPILL SHIELD & 262569 & $1996 / 08 / 21$ & 129.00 & .00 & .00 & 129.00 \\
\hline REPAIR TYPE TOTAL & & & 129.00 & .00 & .00 & 129.00 \\
\hline 07980 DUMP BODY TAILOATE & 259063 & $1996 / 03 / 20$ & 64.50 & .00 & .00 & 64.50 \\
\hline REPAIR TYPE TOTAL & & & 64.50 & .00 & .00 & 64.50 \\
\hline REPAIR SUB GROUP TOTAL & & & 236.50 & .00 & .00 & 236.50 \\
\hline REPAIR GROUP TOTAL & & & 580.50 & 42.73 & 47.83 & 671.06 \\
\hline
\end{tabular}


VEHICLE 3221 INT' ETHANOL TANDEM AXLE

\begin{tabular}{|c|c|c|c|c|c|c|}
\hline $\begin{array}{l}\text { REPAIR } \\
\text { CODE }\end{array}$ & $\begin{array}{l}\text { WORK } \\
\text { ORDER }\end{array}$ & $\begin{array}{c}\text { WO } \\
\text { COMPLETED }\end{array}$ & $\begin{array}{r}\text { LABOR } \\
\text { COST }\end{array}$ & $\begin{array}{r}\text { PARTS } \\
\text { COST }\end{array}$ & $\begin{array}{l}\text { OUTSIDE } \\
\text { COST }\end{array}$ & $\begin{array}{l}\text { TOTAL } \\
\text { COST }\end{array}$ \\
\hline REPAIR SUB GROUP TOTAL & & & 118.25 & .00 & 61.09 & 179.34 \\
\hline REPAIR GROUP TOTAL & & & 118.25 & .00 & 61.09 & 179.34 \\
\hline $7301 \mathrm{~L}$ HYD LINE, PRESSURE & 256395 & $1995 / 12 / 15$ & 43.00 & .00 & .00 & 43.00 \\
\hline REPAIR TYPE TOTAL & & & 43.00 & .00 & .00 & 43.00 \\
\hline REPAIR SUB GROUP TOTAL & & & 43.00 & .00 & .00 & 43.00 \\
\hline 7601R QUICK DISCONNECT & 252342 & $1995 / 06 / 22$ & 21.50 & .00 & .00 & 21.50 \\
\hline REPAIR TYPE TOTAL & & & 21.50 & .00 & .00 & 21.50 \\
\hline REPAIR SUB GROUP TOTAL & & & 21.50 & .00 & .00 & 21.50 \\
\hline 7721R HYD TANK FILTER & 253217 & $1995 / 08 / 17$ & 32.25 & 6.49 & .00 & 38.74 \\
\hline REPAIR TYPE TOTAL & & & 32.25 & 6.49 & .00 & 38.74 \\
\hline REPAIR SUB GROUP TOTAL & & & 32.25 & 6.49 & .00 & 38.74 \\
\hline REPAIR GROUP TOTAL & & & 96.75 & 6.49 & .00 & 103.24 \\
\hline GENERAL GREASING & M06595 & $1995 / 01 / 18$ & 107.50 & 1.74 & 6.56 & 115.80 \\
\hline 98200 GENERAL GREASING & M06919 & $1995 / 03 / 15$ & 86.00 & .87 & 4.92 & 91.79 \\
\hline 98200 GENERAL GREASING & $M 07392$ & $1995 / 07 / 21$ & 86.00 & .88 & .00 & 86.88 \\
\hline 98200 GENERAL GREASING & $M 07632$ & $1995 / 09 / 18$ & 66.00 & .88 & .00 & 86.88 \\
\hline 98200 GENERAL GREASING & M07773 & $1995 / 10 / 24$ & 86.00 & 21.60 & .00 & 107.60 \\
\hline 98200 GENERAL GREASING & M07962 & $1995 / 12 / 07$ & B6.00 & .88 & .00 & 86.88 \\
\hline REPAIR TYPE TOTAI & & & 537.50 & 26.85 & 11.48 & 575.83 \\
\hline $9860 \mathrm{I}$ TRUCK INSPECTION & 253217 & $1995 / 08 / 17$ & 86.00 & .00 & .00 & 86.00 \\
\hline REPAIR TYPE TOTAL & & & 86.00 & .00 & .00 & 86.00 \\
\hline $9890 I$ DIAGNOSTIC ELECTRIC & 254670 & $1995 / 10 / 16$ & 193.50 & .00 & .00 & 193.50 \\
\hline REPAIR TYPE TOTAL & & & 193.50 & .00 & .00 & 193.50 \\
\hline 98951 DIAGNOSTIC MECHANICL & 248654 & $1995 / 01 / 25$ & 64.50 & .00 & .00 & 64.50 \\
\hline REPAIR TYPE TOTAL & & & 64.50 & .00 & .00 & 64.50 \\
\hline REPAIR SUB GROUP TOTAL & & & 881.50 & 26.85 & 11.48 & 919.83 \\
\hline POWER PLANT GROUP & 253475 & $1995 / 08 / 21$ & .00 & .00 & .00 & .00 \\
\hline 99400 POWER PLANT GROUP & 254670 & $1995 / 10 / 16$ & .00 & .00 & 160.08 & 160.08 \\
\hline REPAIR TYPE TOTAL & & & .00 & .00 & 160.08 & 160.08 \\
\hline REPAIR SUB GROUP TOTAL & & & .00 & .00 & 160.08 & 160.08 \\
\hline REPAIR GROUP TOTAL & & & 881.50 & 26.85 & 171.56 & $1,079.91$ \\
\hline IM/MINUS IM TOOTA & & & 4.894 .69 & 420.24 & 683.20 & $5,998.13$ \\
\hline
\end{tabular}


VEHICLE 3221 INT' ETHANOL TANDEM AXLE

\begin{tabular}{|c|c|c|c|c|c|c|}
\hline $\begin{array}{l}\text { REPAIR } \\
\text { CODE }\end{array}$ & $\begin{array}{l}\text { WORK } \\
\text { ORDER }\end{array}$ & $\begin{array}{c}\text { ผо } \\
\text { COMPLETED }\end{array}$ & $\begin{array}{r}\text { LABOR } \\
\text { COST }\end{array}$ & $\begin{array}{r}\text { PARTS } \\
\text { COST }\end{array}$ & $\begin{array}{l}\text { OUTSIDE } \\
\text { COST }\end{array}$ & $\begin{array}{r}\text { TOTAL } \\
\text { COST }\end{array}$ \\
\hline OIL FILLER/DIPSTICK & 251777 & $1995 / 06 / 02$ & 10.75 & .00 & 12.88 & 23.63 \\
\hline OIL FILLER/DIPSTICK & 254670 & $1995 / 10 / 16$ & 10.75 & .00 & 13.29 & 24.04 \\
\hline REPAIR TYPE TOTAL & & & 21.50 & .00 & 26.17 & $\$ 7.67$ \\
\hline 45700 VENTILATING SYSTEM & 250538 & $1995 / 04 / 14$ & 107.50 & 1.21 & 5.02 & 113.73 \\
\hline REPAIR TYPE TOTAL & & & 207.50 & 1.21 & 5.02 & 113.73 \\
\hline 4575R VALVE COVER GASKET & 251777 & $1995 / 06 / 02$ & 10.75 & .00 & 6.48 & 17.23 \\
\hline 4575R VALVE COVER GASKET & 254670 & $1995 / 10 / 16$ & .00 & .00 & 13.36 & 13.36 \\
\hline REPAIR TYPE TOTAL & & & 10.75 & .00 & 19.84 & 30.59 \\
\hline REPAIR SUB GROUP TOTAL & & & 279.50 & 1.21 & 86.44 & 367.15 \\
\hline REPAIR GROUP TOTAL & & & 1.247 .00 & 63.58 & 304.57 & $1,615.15$ \\
\hline TARPALIN ASSEMBLY & 248761 & $1995 / 02 / 03$ & 172.00 & .00 & .00 & 172.00 \\
\hline 51100 TARPALIN ASSEMBLY & 250173 & $1995 / 03 / 24$ & 21.50 & .00 & .00 & 21.50 \\
\hline TARPALIN ASSEMBLX & 252049 & $1995 / 06 / 12$ & 21.50 & .00 & .00 & 21.50 \\
\hline 51100 TARPALIN ASSEMBLY & 253458 & $1995 / 08 / 21$ & 43.00 & .00 & .00 & 43.00 \\
\hline 51100 TARPALIN ASSEMBLY & 254378 & $1995 / 09 / 22$ & 10.75 & .00 & .00 & 10.75 \\
\hline REPAIR TYPE TOTAL & & & 268.75 & .00 & .00 & 268.75 \\
\hline 5115R W/WASHER BLADES & 256054 & $1995 / 11 / 30$ & 21.50 & .00 & .00 & 21.50 \\
\hline REPAIR TYPE TOTAL & & & 21.50 & .00 & .00 & 21.50 \\
\hline REPAIR SUB GROUP TOTAC & & & 290.25 & .00 & .00 & 290.25 \\
\hline $5221 \mathrm{R}$ RADIO, AM/FM & 252440 & $1995 / 08 / 07$ & .00 & .00 & 104.00 & 104.00 \\
\hline 52210 RADIO,AM/FM & 252440 & $1995 / 08 / 07$ & 161.25 & .00 & .00 & 261.25 \\
\hline REPAIR TYPE TOTAL & & & 161.25 & .00 & 104.00 & 265.25 \\
\hline BOX SHAKER & 253458 & $1995 / 08 / 21$ & 75.25 & .00 & .00 & 75.25 \\
\hline 52400 BOX SHAKER & 253526 & $1995 / 08 / 21$ & 86.00 & .00 & .00 & 86.00 \\
\hline REPAIR TYPE TOTAL & & & 161.25 & .00 & .00 & 161.25 \\
\hline 52440 BOX SPRAY PUMP & 252113 & $1995 / 06 / 15$ & 21.50 & .00 & .00 & 21.50 \\
\hline REPAIR TYPE TOTAL & & & 21.50 & .00 & .00 & 21.50 \\
\hline REPAIR SUB GROUP TOTAL & & & 344.00 & .00 & 104.00 & 448.00 \\
\hline 54100 SNOW PLOW FRAME & 256704 & $1995 / 12 / 28$ & 43.00 & .00 & .00 & 43.00 \\
\hline REPAIR TYPE TOTAL & & & 43.00 & .00 & .00 & 43.00 \\
\hline REFAIR SUB GROUP TOTAL & & & 43.00 & .00 & .00 & 43.00 \\
\hline REPAIR GROUP TOTAL & & & 677.25 & .00 & 104.00 & 781.25 \\
\hline 66000 SP EQUIP ACCESSORIES & 252153 & $1995 / 06 / 19$ & .00 & .00 & 20.28 & 20.28 \\
\hline 66000 SP EQUIP ACCESSORIES & 252440 & $1995 / 08 / 07$ & 118.25 & .00 & 40.81 & 159.06 \\
\hline REPAIR TYPE TOTAL & & & 118.25 & .00 & 61.09 & 179.34 \\
\hline
\end{tabular}


VEHICLE 3221 INT' ETHANOL TANDEM AXLE

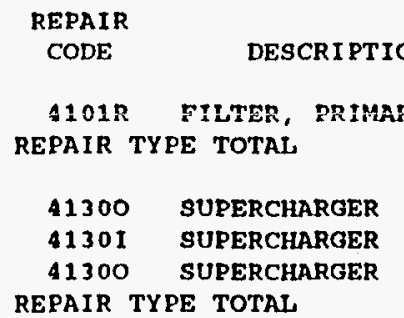

REPAIR SUB GROUP TOTAL

42001 COOLING SYSTEM REPAIR TYPE TOTAL

4212R COOLANT FILTER 4212R COOLANT FILTER REPAIR TYPE TOTAI

REPAIR SUB GROUP TOTAL

43001 EXHAUST SYSTEM REPAIR TYPE TOTAL

43110 TAIL PIPE REPAIR TYPE TOTAL

REPAIR SUB GROUP TOTAL

$$
\begin{array}{lll}
4410 R & \text { FILTER, } \\
4410 R & \text { FILTER, PRIMARY } \\
4410 R & \text { FILTER, PRIMARY } \\
4410 R & \text { FILTER, PRIMARY }
\end{array}
$$
REPAIR TYPE TOTAL

4411R FILTER, SECONDARY REPAIR TYPE TOTAL

4415R FUEL LINES REPNIR TYPE TOTAL

44201 FUEL PUMP REPAIR TYPE TOTAL

REPAIR SUB GROUP TOTAI

\begin{tabular}{|c|c|c|c|c|c|}
\hline $\begin{array}{r}\text { WORK } \\
\text { ORDER }\end{array}$ & $\begin{array}{c}\text { พo } \\
\text { COMPLETED }\end{array}$ & $\begin{array}{l}\text { LABOR } \\
\text { COST }\end{array}$ & $\begin{array}{r}\text { PARTS } \\
\text { COST }\end{array}$ & $\begin{array}{l}\text { OUTSIDE } \\
\text { CosT }\end{array}$ & $\begin{array}{r}\text { TOTAL } \\
\text { COST }\end{array}$ \\
\hline \multirow[t]{2}{*}{250538} & $1995 / 04 / 14$ & 21.50 & .00 & .00 & 21.50 \\
\hline & & 21.50 & .00 & .00 & 21.50 \\
\hline 250538 & $1995 / 04 / 14$ & 96.75 & .00 & .00 & 96.75 \\
\hline 254670 & $1995 / 10 / 16$ & 86.00 & .00 & .00 & 86.00 \\
\hline \multirow[t]{3}{*}{254670} & $1995 / 10 / 16$ & 139.75 & .00 & .00 & 139.75 \\
\hline & & 322.50 & .00 & .00 & 322.50 \\
\hline & & 408.50 & 5.37 & .00 & 413.87 \\
\hline \multirow[t]{2}{*}{252964} & $1995 / 07 / 24$ & 10.75 & .00 & .00 & 10.75 \\
\hline & & 10.75 & .00 & .00 & 10.75 \\
\hline 253217 & $1995 / 08 / 17$ & 32.25 & 8.88 & .00 & 41.13 \\
\hline \multirow[t]{3}{*}{253475} & $1995 / 08 / 21$ & .00 & 8.88 & .00 & 8.88 \\
\hline & & 32.25 & 17.76 & .00 & 50.01 \\
\hline & & 43.00 & 17.76 & .00 & 60.76 \\
\hline \multirow[t]{2}{*}{254378} & $1995 / 09 / 22$ & 10.75 & .00 & .00 & 10.75 \\
\hline & & 10.75 & .00 & .00 & 10.75 \\
\hline \multirow[t]{3}{*}{254378} & $1995 / 09 / 22$ & 32.25 & 30.72 & .00 & 62.97 \\
\hline & & 32.25 & 30.72 & .00 & 62.97 \\
\hline & & 43.00 & 30.72 & .00 & 73.72 \\
\hline 249418 & $1995 / 02 / 27$ & 86.00 & .00 & .00 & 86.00 \\
\hline 252144 & $1995 / 06 / 15$ & 53.75 & .00 & .00 & 53.75 \\
\hline 254552 & $1995 / 10 / 03$ & 43.00 & .00 & 72.71 & 115.71 \\
\hline \multirow[t]{2}{*}{256288} & $1995 / 12 / 15$ & 64.50 & .00 & 72.71 & 137.21 \\
\hline & & 247.25 & .00 & 145.42 & 392.67 \\
\hline \multirow[t]{2}{*}{256288} & $1995 / 12 / 15$ & .00 & .00 & 72.71 & 72.71 \\
\hline & & .00 & .00 & 72.71 & 72.71 \\
\hline \multirow[t]{2}{*}{255670} & $1995 / 11 / 16$ & 150.50 & 8.52 & .00 & 159.02 \\
\hline & & 150.50 & 8.52 & .00 & 159.02 \\
\hline \multirow[t]{3}{*}{254670} & $1995 / 10 / 16$ & 75.25 & .00 & .00 & 75.25 \\
\hline & & 75.25 & .00 & .00 & 75.25 \\
\hline & & $\$ 73.00$ & 8.52 & 218.13 & 699.65 \\
\hline 251777 & $1995 / 06 / 02$ & 32.25 & .00 & .00 & 32.25 \\
\hline \multirow[t]{2}{*}{254670} & $1995 / 10 / 16$ & 64.50 & .00 & 35.41 & 99.91 \\
\hline & & 96.75 & .00 & 35.41 & 132.16 \\
\hline \multirow[t]{2}{*}{255927} & $1995 / 11 / 28$ & 43.00 & .00 & .00 & 43.00 \\
\hline & & 43.00 & .00 & .00 & 43.00 \\
\hline
\end{tabular}

45001 ENGINE
45000 ENGINE
REPNIR TYPE TOTAL

45620 OIL FILTER ASSEMBLY REPAIR TYPE TOTAL 


\section{VEHICLE 3221 INT' ETHANOL TANDEM AXLE}

\begin{tabular}{|c|c|c|c|c|c|c|}
\hline $\begin{array}{l}\text { REPAIR } \\
\text { CODE }\end{array}$ & $\begin{array}{r}\text { WORK } \\
\text { ORDER }\end{array}$ & $\begin{array}{c}\text { WO } \\
\text { COMPLETED }\end{array}$ & $\begin{array}{r}\text { LABOR } \\
\text { COST }\end{array}$ & $\begin{array}{r}\text { PARTS } \\
\text { COST }\end{array}$ & $\begin{array}{l}\text { OUTSIDE } \\
\text { COST }\end{array}$ & $\begin{array}{r}\text { TOTAL } \\
\text { COST }\end{array}$ \\
\hline ALTERNATOR & 254552 & $1995 / 10 / 03$ & 21.50 & .00 & .00 & 21.50 \\
\hline REPAIR TYPE TOTAL & & & 21.50 & .00 & .00 & 21.50 \\
\hline ALTERNATOR BELT & 252914 & $1995 / 07 / 20$ & 86.00 & .00 & .00 & 86.00 \\
\hline REPAIR TYPE TOTAL & & & 86.00 & .00 & .00 & 86.00 \\
\hline REPAIR SUB GROUP TOTAL & & & 107.50 & .00 & .00 & 107.50 \\
\hline 32018 BATTERY & 252813 & $1995 / 07 / 14$ & 161.25 & 164.08 & .00 & 325.33 \\
\hline BATTERY & 254552 & $1995 / 10 / 03$ & 75.25 & .00 & .00 & 75.25 \\
\hline REPAIR TYPE TOTAL & & & 236.50 & 164.08 & .00 & 400.58 \\
\hline BATTERY CABLE & 254552 & $1995 / 10 / 03$ & 53.75 & .00 & .00 & 53.75 \\
\hline BATTERY CABLE & 254670 & $1995 / 10 / 16$ & 86.00 & .00 & .00 & 86.00 \\
\hline REPAIR TYPE TOTAL & & & 139.75 & .00 & .00 & 139.75 \\
\hline REPAIR SUB GROUP TOTAL & & & 376.25 & 164.08 & .00 & 540.33 \\
\hline 3325 I GLOW PLUG(S) & 253458 & $1995 / 08 / 21$ & 107.50 & .00 & .00 & 107.50 \\
\hline REPAIR TYPE TOTAL & & & 107.50 & .00 & .00 & 107.50 \\
\hline REPAIR SUB GROUP TOTAL & & & 107.50 & .00 & .00 & 107.50 \\
\hline HEADLAMP & 256769 & $1995 / 12 / 28$ & 21.50 & .00 & .00 & 21.50 \\
\hline 34010 HEADLAMP & 256716 & $1995 / 12 / 28$ & 53.75 & 12.36 & .00 & 66.11 \\
\hline REPAIR TYPE TOTAL & & & 75.25 & 12.36 & .00 & 87.61 \\
\hline 34150 TURNS IGNAL LAMP & 250657 & $1995 / 04 / 18$ & 311.75 & 8.88 & .00 & 320.63 \\
\hline 3415R TURNSIGNAL LAMP & 256716 & $1995 / 12 / 28$ & .00 & .34 & .00 & .34 \\
\hline REPAIR TYPE TOTAL & & & 311.75 & 9.22 & .00 & 320.97 \\
\hline CLEARANCE/MARKER IMP & 250538 & $1995 / 04 / 14$ & 10.75 & .00 & .00 & 10.75 \\
\hline 34300 CLEARANCE/MARKER IMP & 255697 & $1995 / 11 / 16$ & 21.50 & 12.79 & .00 & 34.29 \\
\hline 34300 CLEARANCE/MARKER LMP & 255927 & $1995 / 11 / 28$ & 21.50 & 1.95 & .00 & 23.45 \\
\hline CLEARANCE/MARKER LMP & 255901 & $1995 / 11 / 28$ & 86.00 & 1.49 & .00 & 87.49 \\
\hline REPAIR TYPE TOTAL & & & 139.75 & 16.23 & .00 & 155.98 \\
\hline 34330 MISC. LAMPS & 256654 & $1995 / 12 / 19$ & 86.00 & .00 & .00 & 86.00 \\
\hline REPAIR TYPE TOTAL & & & 86.00 & .00 & .00 & 86.00 \\
\hline STROBE LAMP & 249853 & $1995 / 03 / 10$ & 86.00 & .00 & .00 & 86.00 \\
\hline REPAIR TYPE TOTAL & & & 86.00 & .00 & .00 & 86.00 \\
\hline REPAIR SUB GROUP TOTAL & & & 698.75 & 37.81 & .00 & 736.56 \\
\hline REPAIR GROUP TOTAL & & & $1,290.00$ & 201.89 & .00 & $1,491.89$ \\
\hline INTAKE SYSTEM & 250538 & $1995 / 04 / 14$ & 64.50 & 5.37 & .00 & 69.87 \\
\hline REPAIR TYPE TOTAL & & & 64.50 & 5.37 & .00 & 69.87 \\
\hline
\end{tabular}


VEHICLE 3221 INT' ETHANOL TANDEM AXLE

\begin{tabular}{|c|c|c|c|c|c|c|}
\hline $\begin{array}{l}\text { REPAIR } \\
\text { CODE } \quad \text { DESCRIPTION }\end{array}$ & $\begin{array}{r}\text { WORK } \\
\text { ORDER }\end{array}$ & $\begin{array}{l}\text { WO } \\
\text { COMPLETED }\end{array}$ & $\begin{array}{l}\text { LABOR } \\
\text { COST }\end{array}$ & $\begin{aligned} \text { PARTS } \\
\text { COST }\end{aligned}$ & $\begin{array}{l}\text { OUTSIDE } \\
\text { COST }\end{array}$ & $\begin{array}{l}\text { TOTAL } \\
\text { COST }\end{array}$ \\
\hline ENDYR TOTAL & & & 7.285 .75 & 355.54 & 2.045 .90 & 9.588 .13 \\
\hline $1115 \mathrm{~L}$ & 250538 & $1995 / 04 / 14$ & 118.25 & 10.97 & 44.20 & 173.42 \\
\hline 1115L PMA & 256654 & $1995 / 12 / 19$ & 175.44 & 10.98 & 49.20 & 235.62 \\
\hline REPAIR TYPE TOTAL & & & 293.69 & 21.95 & 93.40 & 409.04 \\
\hline $1120 \mathrm{~L}$ РMB & 253217 & $1995 / 08 / 17$ & 161.25 & .00 & .00 & 161.25 \\
\hline REPAIR TYPE TOTAL & & & 161.25 & .00 & .00 & 161.25 \\
\hline REPAIR SUB GROUP TOTAL & & & 454.94 & 21.95 & 93.40 & 570.29 \\
\hline REPAIR GROUP TOTAL & & & 454.94 & 21.95 & 93.40 & 570.29 \\
\hline PM/MINUS PM TOTAL & & & 454.94 & 21.95 & 93.40 & 570.29 \\
\hline 0215R MIRROR, EXTERNAL & 254552 & $1995 / 10 / 03$ & 43.00 & 13.81 & .00 & 56.81 \\
\hline REPAIR TYPE TOTAL & & & 43.00 & 13.81 & .00 & 56.81 \\
\hline REPAIR SUB GROUP TOTAL & & & 43.00 & 13.81 & .00 & 56.81 \\
\hline $07300 \quad$ HOOD & 256769 & $1995 / 12 / 28$ & 64.50 & .00 & .00 & 64.50 \\
\hline REPAIR TYPE TOTAL & & & 64.50 & .00 & .00 & 64.50 \\
\hline 0797R DMPBODY SPILL SHIELD & 252153 & $1995 / 06 / 19$ & 215.00 & 11.77 & 35.66 & 262.43 \\
\hline REPAIR TYPE TOTAL & & & 215.00 & 11.77 & 35.66 & 262.43 \\
\hline REPAIR SUB GROUP TOTAL & & & 279.50 & 11.77 & 35.66 & 326.93 \\
\hline REPAIR GROUP TOTAL & & & 322.50 & 25.58 & 35.66 & 383.74 \\
\hline 17010 TIRE, FLAT & 253019 & $1995 / 07 / 26$ & 86.00 & .00 & .00 & 86.00 \\
\hline 1701R TIRE, FLAT & 254019 & $1995 / 09 / 15$ & 67.94 & 95.85 & .00 & 163.79 \\
\hline REPAIR TYPE TOTAL & & & 153.94 & 95.85 & .00 & 249.79 \\
\hline REPAIR SUB GROUP TOTAL & & & 153.94 & 95.85 & .00 & 249.79 \\
\hline $1821 R$ LUG NUTS & 256113 & $1995 / 12 / 05$ & 43.00 & .00 & 6.32 & 49.32 \\
\hline REPAIR TYPE TOTAL & & & 43.00 & .00 & 6.32 & 49.32 \\
\hline REPAIR SUB GROUP TOTAL & & & 43.00 & .00 & 6.32 & 49.32 \\
\hline REPAIR GROUP TOTAL, & & & 196.94 & 95.85 & 6.32 & 299.11 \\
\hline 26000 TRANSMISSION, MANUAL, & 255927 & $1995 / 11 / 28$ & 64.50 & .00 & .00 & 64.50 \\
\hline REPAIR TYPE TOTAL & & & 64.50 & .00 & .00 & 64.50 \\
\hline REPAIR SUB GROUP TOTAL & & & 64.50 & .00 & .00 & 64.50 \\
\hline REPAIR GROUP TOTAL & & & 64.50 & .00 & .00 & 64.50 \\
\hline
\end{tabular}




\section{VEHICLE 3221 INT' ETHANOL TANDEM AXLE}

\begin{tabular}{|c|c|c|}
\hline $\begin{array}{l}\text { REPAIR } \\
\text { CODE }\end{array}$ & DESCR & IPTION \\
\hline \multicolumn{3}{|c|}{ REPAIR GROUP TOTAL } \\
\hline 73010 & HYD LINE, & PRESSURE \\
\hline $\begin{array}{l}73011 \\
73010\end{array}$ & HYD LINE, & PRESSURE \\
\hline
\end{tabular}
REPAIR TYPE TOTAL.

REPAIR SUB GROUP TOTAL

76010 QUICK DISCONNECT REPAIR TYPE TOTAL

REPAIR SUB GROUP TOTAL

7720R HYD TANK FILTER ASSM REPAIR TYPE TOTAL

T721R HYD TANK FILTER REPAIR TYPE TOTAL

REPAIR SUB GROUP TOTAL

REPAIR GROUP TOTAL

$\begin{array}{ll}98200 & \text { GENERAL GREASING } \\ 98200 & \text { GENERAL GREASING } \\ 98200 & \text { GENERAL GREASING } \\ 98200 & \text { GENERAL GREASING } \\ 98200 & \text { GENERAL GREASING }\end{array}$

98200 GENERAL GREASING REPAIR TYPE TOTAL

98601 TRUCK INSPECTION REPAIR TYPE TOTAL

9895 I DIAGNOSTIC MECHANICT. REPAIR TYPE TOTAL

REPAIR SUB GROUP TOTAL

99400 PONER PLANT GROUP 99400
99400 REPAIR TYPE TOTAL

REPAIR SUE GROUP TOTAL

REPAIR GROUP TOTAL,

PM/MINUS PM TOTAL
WORK

ORDER

พั

COMPLETED

LABOR
COST

PARTS
COST

OUTSIDE

COST

TOTAL

129.00

$242859 \quad 1994 / 06 / 15$

$246436 \quad 1994 / 10 / 28$

86.00

21.50

$246436 \quad 1994 / 10 / 28$

64.50

172.00

172.00

$2430061994 / 06 / 21$

43.00

43.00
43.00

43.00

$244783 \quad 1994 / 09 / 14$

118.25

118.25

$245913 \quad 1994 / 10 / 05$

32.25

32.25

150.50

365.50

M05296 1994/02/22

M05443 1994/03/15

M05661 1994/05/11

M05986 1994/08/20

M06025 1994/09/08

M06448 1994/12/14

$244783 \quad 1994 / 09 / 14$

245913

$1994 / 10 / 05$

2

$1994 / 03 / 09$

240844 $1994 / 04 / 04$

86.00

86.00

1.07 .50

64.50

96.75

86.00

526.75

21.50

21.50

21.50

21.50

569.75

.00

.00
.00

.00

.00

569.75

$6,874.00$
.00

.00

.00

.00

.00
.00

.00

.00

.00
.00

.00

.00

.00

.00

.00

.00

.00

2.56

2.56

1. 28

7.92

5.00

20.28

.00

.00

.00

.00

20.28

4.80

.00

183.37

183.37

183.37

203.65
$334.12 \quad 1,978.16$

129.00

86.00

21.50

64.50

172.00

172.00

43.00
43.00

43.00

120.19

120.19

38.92

38.92

159.11

374.11

89.36

89.36

109.58

73.22

97.55

92.76

551.83

21.50

21.50

21.50
21.50

594.83

.00
183.37

183.37

183.37

183.37

778.20

$9,186.28$ 
VEHICLE 3221 INT' ETHANOL TANDEM AXLE

\begin{tabular}{|c|c|c|c|c|c|c|}
\hline $\begin{array}{l}\text { REPAIR } \\
\text { CODE }\end{array}$ & $\begin{array}{l}\text { WORK } \\
\text { ORDER }\end{array}$ & $\begin{array}{c}\text { Wo } \\
\text { COMPLETED }\end{array}$ & $\begin{array}{l}\text { LABOR } \\
\text { COST }\end{array}$ & $\begin{array}{l}\text { PARTS } \\
\text { COST }\end{array}$ & $\begin{array}{l}\text { OUTS IDE } \\
\text { COST }\end{array}$ & $\begin{array}{l}\text { TOTAL } \\
\text { COST }\end{array}$ \\
\hline$\triangle 4200$ FUEL PUMP & 242400 & $1994 / 06 / 01$ & 139.75 & .93 & .00 & 140.68 \\
\hline REPAIR TYPE TOTAL & & & 139.75 & .93 & .00 & 240.68 \\
\hline REPAIR SUB GROUP TOTAL & & & 473.00 & .93 & 477.09 & 951.02 \\
\hline $\begin{array}{l}\text { 4500I ENGINE } \\
\text { REPAIR TXPE TOTAL }\end{array}$ & 247777 & $1994 / 12 / 30$ & $\begin{array}{l}75.25 \\
75.25\end{array}$ & $\begin{array}{l}.00 \\
.00\end{array}$ & $\begin{array}{l}.00 \\
.00\end{array}$ & $\begin{array}{l}75.25 \\
75.25\end{array}$ \\
\hline VALVETRAIN ASSEMBLY & 244660 & $1994 / 08 / 26$ & 64.50 & .00 & .00 & 64.50 \\
\hline REPAIR TYPE TOTAL & & & 64.50 & .00 & .00 & 64.50 \\
\hline REPAIR SUB GROUP TOTAL & & & 139.75 & .00 & .00 & 139.75 \\
\hline REPAIR GROUP TOTAL & & & $1,575.75$ & 62.06 & 519.31 & $2,157.12$ \\
\hline 51000 ACCESSORIES, GENERAL & 240994 & $1994 / 04 / 12$ & 569.75 & 3.62 & .00 & 573.37 \\
\hline REPAIR TYPE TOTAL & & & 569.75 & 3.62 & .00 & 573.37 \\
\hline MUD FLAP (S) & 243006 & $1994 / 06 / 21$ & 10.75 & .00 & .00 & 10.75 \\
\hline REPAIR TYPE TOTAL & & & 10.75 & .00 & .00 & 10.75 \\
\hline AIR HORN ASSEMBLY & 243583 & $1994 / 07 / 12$ & 258.00 & .00 & 64.76 & 322.76 \\
\hline REPAIR TYPE TOTAL & & & 258.00 & .00 & 64.76 & 322.76 \\
\hline $5110 R$ TARPALIN ASSEMBLY & 240994 & $1994 / 04 / 12$ & .00 & .00 & 852.71 & 852.71 \\
\hline 51100 TARPALIN ASSEMBLY & 246026 & $1994 / 10 / 10$ & 43.00 & .00 & .00 & $\$ 3.00$ \\
\hline REPAIR TYPE TOTAL & & & 43.00 & .00 & 852.71 & 895.71 \\
\hline REPAIR SUB GROUP TOTAL & & . & 881.50 & 3.62 & 917.47 & $1,802.59$ \\
\hline $5244 \mathrm{R}$ BOX SPRAY PUMP & 243726 & $1994 / 07 / 29$ & 645.00 & 22.07 & 182.50 & 849.57 \\
\hline REPAIR TYPE TOTAL & & & 645.00 & 22.07 & 182.50 & 849.57 \\
\hline REPAIR SUB GROUR TOTAL & & & 645.00 & 22.07 & 182.50 & 849.57 \\
\hline 54050 SNOW PLOW LIFT CYLIN & 246993 & $1994 / 11 / 22$ & 21.50 & .00 & .00 & 21.50 \\
\hline REPAIR TYPE TOTAL & & & 21.50 & .00 & .00 & 21.50 \\
\hline 54220 UNDERBODY PLOW RAM & 246993 & $1994 / 11 / 22$ & 129.00 & .00 & .00 & 129.00 \\
\hline REPAIR TYPE TOTAL & & & 129.00 & .00 & .00 & 129.00 \\
\hline REPAIR SUB GROUP TOTAL & & & 150.50 & .00 & .00 & 150.50 \\
\hline REPAIR GROUP TOTAL & & & $1,677.00$ & 25.69 & $1,099.97$ & 2.802 .66 \\
\hline 66000 SP EQUIP ACCESSORIES & 243006 & $1994 / 06 / 21$ & 86.00 & .00 & .00 & 86.00 \\
\hline 66000 SP EQUIP ACCESSORIES & 247224 & $1994 / 12 / 01$ & 43.00 & .00 & .00 & 43.00 \\
\hline REPAIR TYPE TOTAL & & & 129.00 & .00 & .00 & 129.00 \\
\hline REPAIR SUB GROUP TOTAL & & & 129.00 & .00 & .00 & 129.00 \\
\hline
\end{tabular}


VEHICLE 3221 INT' ETHANOL TANDEM AXLE

REPAIR
CODE
REPAIR GROUP TOTAL
42001 COOLING SYSTEM
42001 COOLING SYSTEM
42000 COOLING SYSTEM
REPAIR TYPE TOTAL

42040 RAD HOSE, LOWER REPAIR TYPE TOTAL

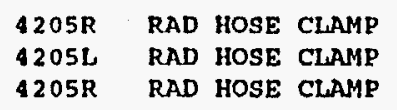
REPAIR TYPE TOTAL

4212R COOLANT FILTER REPAIR TYPE TOTAL

$$
\begin{aligned}
& 42260 \text { FAN CLUTCH } \\
& 42260 \text { FAN CLUTCH } \\
& 42260 \text { FAN CLUTCH } \\
& \text { REPAIR TYPE TOTAL }
\end{aligned}
$$$$
\begin{aligned}
& 4230 R \text { WATER PUMP } \\
& 42301 \text { WNTER PUMP }
\end{aligned}
$$$$
\text { REPAIR TYPE TOTAL }
$$

42310 WATER PUMP BELT REPAIR TYPE TOTAL

REPAIR SUB GROUP TOTAL

$$
43500 \text { CLAMPS }
$$

\begin{tabular}{|c|c|c|c|}
\hline \multirow[t]{2}{*}{ ORDER } & $\begin{array}{c}\text { Wo } \\
\text { COMPLETED }\end{array}$ & $\begin{array}{r}\text { LABOR } \\
\text { COSTT }\end{array}$ & $\begin{array}{r}\text { PARTS } \\
\text { COST }\end{array}$ \\
\hline & & 579.00 & 224. \\
\hline 245792 & $1994 / 09 / 29$ & 75.25 & \\
\hline 246495 & $1994 / 10 / 31$ & 53.75 & .0 \\
\hline \multirow[t]{2}{*}{246495} & $1994 / 10 / 31$ & 21.50 & .0 \\
\hline & & 150.50 & . \\
\hline 246495 & $1994 / 10 / 31$ & $\begin{array}{l}64.50 \\
64.50\end{array}$ & \\
\hline 245792 & $1994 / 09 / 29$ & 86.00 & \\
\hline 245792 & $1994 / 09 / 29$ & 32.25 & \\
\hline \multirow[t]{2}{*}{246495} & $1994 / 10 / 31$ & .00 & . \\
\hline & & 118.25 & .0 \\
\hline 244783 & $1994 / 09 / 14$ & $\begin{array}{l}53.75 \\
53.75\end{array}$ & $\begin{array}{l}44.7 \\
44.7\end{array}$ \\
\hline 238557 & $1994 / 01 / 24$ & 60.00 & \\
\hline 244660 & $1994 / 08 / 26$ & 75.25 & \\
\hline \multirow[t]{2}{*}{244783} & $1994 / 09 / 14$ & 32.25 & \\
\hline & & 167.50 & \\
\hline \multirow{3}{*}{$\begin{array}{l}246522 \\
246522\end{array}$} & $1994 / 11 / 17$ & 225.75 & \\
\hline & $1994 / 11 / 17$ & 75.25 & \\
\hline & & 301.00 & \\
\hline \multirow[t]{3}{*}{239135} & $1994 / 02 / 04$ & 32.25 & \\
\hline & & 32.25 & \\
\hline & & 887.75 & 44.7 \\
\hline \multirow[t]{3}{*}{248025} & $1994 / 12 / 30$ & 75.25 & \\
\hline & & 75.25 & 16. \\
\hline & & 75.25 & 16.3 \\
\hline \multirow[t]{2}{*}{238696} & $1994 / 01 / 25$ & 86.00 & \\
\hline & & 86.00 & \\
\hline 238220 & $1994 / 01 / 10$ & 64.50 & .0 \\
\hline 241536 & $1994 / 04 / 25$ & 21.50 & \\
\hline 243006 & $1994 / 06 / 21$ & 64.50 & 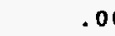 \\
\hline 246263 & $1994 / 10 / 20$ & 43.00 & \\
\hline \multirow[t]{2}{*}{247515} & $1994 / 12 / 12$ & 53.75 & \\
\hline & & 247.25 & \\
\hline \multirow[t]{2}{*}{238696} & $1994 / 01 / 25$ & .00 & .0 \\
\hline & & .00 & .0 \\
\hline
\end{tabular}$$
\text { REPAIR TYPE TOTAL }
$$

\begin{tabular}{|c|c|}
\hline $\begin{array}{l}\text { OUTSIDE } \\
\text { COST }\end{array}$ & $\begin{array}{r}\text { TotaL } \\
\text { COST }\end{array}$ \\
\hline .00 & 803.65 \\
\hline .00 & 75.25 \\
\hline .00 & 53.75 \\
\hline .00 & 21.50 \\
\hline .00 & 150.50 \\
\hline .00 & 64.50 \\
\hline .00 & 64.50 \\
\hline 4.22 & 90.22 \\
\hline .00 & 32.25 \\
\hline 38.00 & 38.00 \\
\hline 42.22 & 160.47 \\
\hline .00 & 98.49 \\
\hline .00 & 98.49 \\
\hline .00 & 60.00 \\
\hline .00 & 75.25 \\
\hline .00 & 32.25 \\
\hline .00 & 167.50 \\
\hline .00 & 225.75 \\
\hline .00 & 75.25 \\
\hline .00 & 301.00 \\
\hline .00 & 32.25 \\
\hline .00 & 32.25 \\
\hline 42.22 & 974.71 \\
\hline .00 & 91.64 \\
\hline .00 & 91.64 \\
\hline .00 & 91.64 \\
\hline .00 & 86.00 \\
\hline .00 & 86.00 \\
\hline 145.42 & 209.92 \\
\hline .00 & 21.50 \\
\hline 136.54 & 201.04 \\
\hline 145.42 & 188.42 \\
\hline .00 & 53.75 \\
\hline 427.38 & 674.63 \\
\hline 49.71 & 49.71 \\
\hline 49.71 & 49.71 \\
\hline
\end{tabular}

REPAIR SUB GROUP TOTAL

$$
44010 \text { TANK, DIESEL }
$$$$
\text { REPAIR TYPE TOTAL }
$$

$$
\begin{array}{ll}
\text { 4410R } & \text { FILTER, PRIMARY } \\
4410 R & \text { FILTER, PRIMARY } \\
4410 R & \text { FILTER, PRIMARY } \\
4410 R & \text { FILTER, PRIMARY } \\
4410 R & \text { FILTER, PRIMARY } \\
\text { REPAIR TYPE TOTAL } &
\end{array}
$$

4411R FILTER, SECONDARY REPAIR TYPE TOTAL, 
VEHICLE 3221 INT' ETHANOL TANDEM AXLE

\begin{tabular}{|c|c|c|c|c|c|c|}
\hline $\begin{array}{l}\text { REPAIR } \\
\text { CODE }\end{array}$ & $\begin{array}{l}\text { WORK } \\
\text { ORDER }\end{array}$ & $\begin{array}{c}\text { WO } \\
\text { COMPLETED }\end{array}$ & $\begin{array}{l}\text { LAABOR } \\
\text { COST }\end{array}$ & $\begin{array}{l}\text { PARTS } \\
\text { COST }\end{array}$ & $\begin{array}{l}\text { OUTSIDE } \\
\text { COST }\end{array}$ & $\begin{array}{l}\text { TOTAL } \\
\text { COST }\end{array}$ \\
\hline $\begin{array}{l}17010 \text { TIRE, FLAT } \\
\text { REPAIR TYPE TOTAL }\end{array}$ & 246993 & $1994 / 11 / 22$ & $\begin{array}{l}43.00 \\
43.00\end{array}$ & $\begin{array}{l}.00 \\
.00\end{array}$ & $\begin{array}{l}.00 \\
.00\end{array}$ & $\begin{array}{l}43.00 \\
43.00\end{array}$ \\
\hline REPAIR SUB GROUP TOTAL & & & 43.00 & .00 & .00 & 43.00 \\
\hline $18010 \quad$ RIM & 246522 & $1994 / 11 / 17$ & 215.00 & .00 & 26.66 & 241.66 \\
\hline REPAIR TYPE TOTAL & & & 215.00 & .00 & 26.66 & 241.66 \\
\hline REPAIR SUB GROUP TOTAL & & & 215.00 & .00 & 26.66 & 241.66 \\
\hline REPAIR GROUP TOTAL & & & 559.00 & 4.24 & 82.17 & 645.41 \\
\hline $\begin{array}{l}2300 \mathrm{~L} \text { CLUTCH ASSEMBLY } \\
\text { REPAIR TXPE TOTAL }\end{array}$ & 244783 & $1994 / 09 / 14$ & $\begin{array}{l}64.50 \\
64.50\end{array}$ & $\begin{array}{l}.00 \\
.00\end{array}$ & $\begin{array}{l}.00 \\
.00\end{array}$ & $\begin{array}{l}64.50 \\
64.50\end{array}$ \\
\hline REPAIR SUB GROUP TOTAL & & & 64.50 & .00 & .00 & 64.50 \\
\hline REPAIR GROUP TOTAL & & & 64.50 & .00 & .00 & 64.50 \\
\hline 32010 BATTERY & 238879 & $1994 / 01 / 25$ & 21.50 & 66.42 & .00 & 87.92 \\
\hline 3201R BATTERY & 247777 & $1994 / 12 / 30$ & 10.75 & 70.04 & .00 & 80.79 \\
\hline REPAIR TYPE TOTAL & & & 32.25 & 136.46 & .00 & 168.71 \\
\hline 32020 BATTERY CABLE & 238879 & $1994 / 01 / 25$ & 43.00 & 9.24 & .00 & 52.24 \\
\hline REPAIR TYPE TOTAL & & & 43.00 & 9.24 & .00 & 52.24 \\
\hline 32060 BATTERY HOLDOWNS & 238879 & $1994 / 01 / 25$ & 43.00 & 1.48 & .00 & 44.48 \\
\hline REPAIR TYPE TOTAL & & & 43.00 & 1.48 & .00 & 44.48 \\
\hline REPAIR SUB GROUP TOTAL & & & 118.25 & 147.18 & .00 & 265.43 \\
\hline $\begin{array}{ll}3401 L & \text { HEADLAMP } \\
3401 R & \text { HEADLAMMP }\end{array}$ & $\begin{array}{l}238557 \\
247185\end{array}$ & $\begin{array}{l}1994 / 01 / 24 \\
1994 / 11 / 29\end{array}$ & $\begin{array}{l}20.00 \\
32.25\end{array}$ & $\begin{array}{r}.00 \\
10.87\end{array}$ & $\begin{array}{l}.00 \\
.00\end{array}$ & $\begin{array}{l}20.00 \\
43.12\end{array}$ \\
\hline REPAIR TYPE TOTAL & & & 52.25 & 10.87 & .00 & 63.12 \\
\hline 34150 TURNSIGNAL LAMP & 239274 & $1994 / 03 / 29$ & 43.00 & .00 & .00 & 43.00 \\
\hline REPAIR TYPE TOTAL & & & 43.00 & .00 & .00 & 43.00 \\
\hline CLEARANCE/MARKER LMP & 240567 & $1994 / 03 / 18$ & 21.50 & .00 & .00 & 21.50 \\
\hline $3430 R$ CLEARANCE/MARKER LMP & 243006 & $1994 / 06 / 21$ & 64.50 & 10.41 & .00 & 74.91 \\
\hline REPAIR TYPE TOTAL & & & 86.00 & 10.41 & .00 & 96.41 \\
\hline 34400 STROBE LAMP & 247224 & $1994 / 12 / 01$ & 139.75 & 37.80 & .00 & 177.55 \\
\hline REPAIR TYPE TOTAL & & & 139.75 & 37.80 & .00 & 177.55 \\
\hline 3450R SANDER/WORK LAMP & 246757 & $1994 / 11 / 10$ & 86.00 & 9.16 & .00 & 95.16 \\
\hline 3450R SANDER/WORK LAMP & 247127 & $1994 / 11 / 29$ & 53.75 & 9.23 & .00 & 62.98 \\
\hline REPAIR TYPE TOTAL & & & 139.75 & 18.39 & .00 & 158.14 \\
\hline REPAIR SUB GROUP TOTAL & & & 75 & 77.47 & .00 & 538.22 \\
\hline
\end{tabular}




\begin{tabular}{|c|c|c|c|c|c|c|}
\hline $\begin{array}{l}\text { REPAIR } \\
\text { CODE } \quad \text { DESCRIPTION }\end{array}$ & $\begin{array}{l}\text { WORK } \\
\text { ORDER }\end{array}$ & $\begin{array}{c}\text { WO } \\
\text { COMPLETED }\end{array}$ & $\begin{array}{r}\text { LABOR } \\
\text { COST }\end{array}$ & $\begin{array}{r}\text { PARTS } \\
\text { COST }\end{array}$ & $\begin{array}{l}\text { OUTS IDE } \\
\text { COST }\end{array}$ & $\begin{array}{l}\text { TOTAL } \\
\text { COST }\end{array}$ \\
\hline REPAIR SUB GROUP TOTAL & & & 311.75 & 22.42 & 67.74 & 401.91 \\
\hline REPAIR GROUP TOTAL & & & 311.75 & 22.42 & 67.74 & 401.91 \\
\hline PM/MINUS PM TOTAL & & & 311.75 & 22.42 & 67.74 & 401.91 \\
\hline 01530 HEATER SHUTOFF VALVE & 242292 & $1994 / 05 / 23$ & 53.75 & .00 & .00 & 53.75 \\
\hline REPAIR TYPE TOTAL & & & 53.75 & .00 & .00 & 53.75 \\
\hline REPAIR SUB GROUP TOTAL & & & 53.75 & .00 & .00 & 53.75 \\
\hline O3OOR GAUGES/INSTRUMENTS & 240614 & $1994 / 05 / 04$ & 731.00 & 4.07 & 17.98 & 753.05 \\
\hline REPAIR TYPE TOTAL & & & 731.00 & 4.07 & 17.98 & 753.05 \\
\hline 03250 FUEL GAUGE & 239117 & $1994 / 02 / 14$ & 129.00 & .00 & .00 & 129.00 \\
\hline REPAIR TYPE TOTAL & & & 229.00 & .00 & .00 & 129.00 \\
\hline FUEL GG SENDER UNIT & 239117 & $1994 / 02 / 14$ & .00 & .00 & 26.18 & 26.18 \\
\hline 03260 FUEL GG SENDER UNIT & 242400 & $1994 / 06 / 01$ & 182.75 & .00 & .00 & 182.75 \\
\hline 0326R FUEL GO SENDER UNIT & 242400 & $1994 / 06 / 01$ & .00 & .00 & 28.90 & 28.90 \\
\hline REPAIR TYPE TOTAL & & & 182.75 & .00 & 55.08 & 237.83 \\
\hline REPAIR SUB GROUP TOTAL & & & $1,042.75$ & 4.07 & 73.06 & $1,119.88$ \\
\hline 07120 DOOR LATCUI MSSEMBLY & 247674 & $1994 / 12 / 19$ & 161.25 & .00 & .00 & 161.25 \\
\hline REPAIR TYIE TOTAL & & & 161.25 & .00 & .00 & 161.25 \\
\hline 07380 ENGINE COVER ( $\mathrm{s}$ ) & 239274 & $1994 / 03 / 29$ & 75.25 & .00 & .00 & 75.25 \\
\hline REPAIR TYPE TOTAL & & & 75.25 & .00 & .00 & 75.25 \\
\hline 07980 DUMP BODY TAILGATE & 246263 & $1994 / 10 / 20$ & 21.50 & .00 & .00 & 21.50 \\
\hline REPAIR TYPE TOTAL & & & 21.50 & .00 & .00 & 21.50 \\
\hline REPAIR SUB GROUP TOTAL & & & 258.00 & .00 & .00 & 258.00 \\
\hline REPAIR GROUP TOTAL & & & $1,354.50$ & 4.07 & 73.06 & $1,431.63$ \\
\hline 13350 BRAKE, POWER ASSIST & 239274 & $1994 / 03 / 29$ & 32.25 & .00 & .00 & 32.25 \\
\hline REPAIR TYPE TOTAL & & & 32.25 & .00 & .00 & 32.25 \\
\hline 13500 AIR COMPRESSOR & 239077 & $1994 / 02 / 01$ & 139.75 & 4.24 & .00 & 143.99 \\
\hline REPAIR TYPE TOTAL & & & 139.75 & 4.24 & .00 & 143.99 \\
\hline 13520 AIR COMPR FILTER & 239517 & $1994 / 02 / 14$ & 129.00 & .00 & .00 & 129.00 \\
\hline REPAIR TYPE TOTAL & & & 129.00 & .00 & .00 & 129.00 \\
\hline 13550 AIR DRYER ASSEMBLY & 239517 & $1994 / 02 / 14$ & .00 & .00 & 55.51 & 55.51 \\
\hline REVIAR 'IYI'E TO'AL, & & & .00 & .00 & 55.51 & 55.51 \\
\hline REPAIR SUB GROUP TOTAL & & & 301.00 & 4.24 & 55.51 & 360.75 \\
\hline
\end{tabular}




\section{VEHICLE 3221 INT' ETHANOL TANDEM AXLE}

\begin{tabular}{|c|c|c|c|c|c|c|}
\hline $\begin{array}{l}\text { REPAIR } \\
\text { CODE }\end{array}$ & $\begin{array}{l}\text { WORK } \\
\text { ORDER }\end{array}$ & $\begin{array}{c}\text { WO } \\
\text { COMPLETED }\end{array}$ & $\begin{array}{r}\text { LABOR } \\
\text { COST }\end{array}$ & $\begin{array}{r}\text { PARTS } \\
\text { COST }\end{array}$ & $\begin{array}{l}\text { OUTSIDE } \\
\text { COST }\end{array}$ & $\begin{array}{l}\text { TOTAL } \\
\text { COST }\end{array}$ \\
\hline AIR HORN ASSEMBLY & 236740 & $1993 / 11 / 12$ & 21.50 & .00 & .00 & 21.50 \\
\hline 51050 AIR HORN ASSEMBLY & 237426 & $1993 / 12 / 03$ & 53.75 & .54 & .00 & 54.29 \\
\hline AIR HORN ASSEMBLY & 238027 & $1993 / 12 / 22$ & 43.00 & .00 & .00 & 43.00 \\
\hline REPAIR TYPE TOTAL & & & 118.25 & .54 & .00 & 118.79 \\
\hline REPAIR SUB GROUP TOTAL & & & 118.25 & .54 & .00 & 118.79 \\
\hline $\begin{array}{l}52000 \text { ACCESSORY, ELECTRIC } \\
\text { REPAIR TYPE TOTAL }\end{array}$ & 236706 & $1993 / 11 / 17$ & $\begin{array}{l}75.25 \\
75.25\end{array}$ & $\begin{array}{l}.00 \\
.00\end{array}$ & $\begin{array}{l}.00 \\
.00\end{array}$ & $\begin{array}{l}75.25 \\
75.25\end{array}$ \\
\hline 52300 RADIO ANTENNA & 236740 & $1993 / 11 / 12$ & 21.50 & .00 & .00 & 21.50 \\
\hline REPAIR TYPE TOTAL & & & 21.50 & .00 & .00 & 21.50 \\
\hline REPAIR SUB GROUP TOTAL & & & 96.75 & .00 & .00 & 96.75 \\
\hline 5910R PINTLE HOOK & 231148 & $1993 / 09 / 28$ & .00 & .00 & 45.12 & 45.12 \\
\hline REPAIR TYPE TOTAL & & & .00 & .00 & 45.12 & 45.12 \\
\hline REPAIR SUB GROUP TOTAL & & & .00 & .00 & 45.12 & 45.12 \\
\hline REPAIR GROUP TOTAL & & & 215.00 & .54 & 45.12 & 260.66 \\
\hline 66000 SP EQUIP ACCESSORIES & 237741 & $1993 / 12 / 22$ & 225.75 & 3.56 & 8.83 & 238.14 \\
\hline REPAIR TYPE TOTAL & & & 225.75 & 3.56 & 8.83 & 238.14 \\
\hline REPAIR SUB GROUP TOTAL & & & 225.75 & 3.56 & 8.83 & 238.14 \\
\hline REPAIR GROUP TOTAL & & & 225.75 & 3.56 & 8.83 & 238.14 \\
\hline NEW VEHICLE PREP. & 231148 & $1993 / 09 / 28$ & $3,033.65$ & 274.44 & 243.73 & 3.551 .82 \\
\hline 98301 NEW VEHICLE PREP. & 231148 & $1993 / 09 / 28$ & .00 & .00 & 271.20 & 271.20 \\
\hline 98300 NEW VEHICLE PREP. & 235740 & $1993 / 10 / 01$ & 75.25 & .00 & 97.65 & 172.90 \\
\hline 98300 NEW VEHICLE PREP. & 236740 & $1993 / 11 / 12$ & .00 & .00 & 316.35 & 316.35 \\
\hline REPAIR TYPE TOTAL & & & $3,108.90$ & 274.44 & 928.93 & $4,312,27$ \\
\hline REPAIR SUB GROUP TOTAL & & & 3.108 .90 & 274.44 & 928.93 & 4.312 .27 \\
\hline REPAIR GROUP TOTAL & & & $3,108.90$ & 274.44 & 928.93 & $4,312.27$ \\
\hline PM/MINUS PM TOTAL & & & $4,764.40$ & 280.69 & $1,513.72$ & 6.558 .81 \\
\hline ENDYR TOTAL & & & 4.764 .40 & 280.69 & $1,513.72$ & 6.558 .81 \\
\hline 11151. PMA & 238557 & $1994 / 01 / 24$ & 150.50 & 11.52 & 20.48 & 182.50 \\
\hline $1115 \mathrm{~L}$ PMA & 239517 & $1994 / 02 / 14$ & .00 & .00 & 2.56 & 2.56 \\
\hline 1115L PMA & 246757 & $1994 / 11 / 10$ & 118.25 & 10.90 & 44.70 & 173.85 \\
\hline RFPAIR TYPE TOTAI, & & & 268.75 & 22.42 & 67.74 & 358.91 \\
\hline $\begin{array}{l}\text { 11201, PMB } \\
\text { REPAIR TYPE TOTAL }\end{array}$ & 244783 & $1994 / 09 / 14$ & $\begin{array}{l}43.00 \\
43.00\end{array}$ & $\begin{array}{l}.00 \\
.00\end{array}$ & $\begin{array}{l}.00 \\
.00\end{array}$ & $\begin{array}{l}43.00 \\
43.00\end{array}$ \\
\hline
\end{tabular}


VEHICLE 3221 INT' ETHANOL TANDEM AKLE

\begin{tabular}{|c|c|c|c|c|c|c|}
\hline $\begin{array}{l}\text { REPAIR } \\
\text { CODE }\end{array}$ & $\begin{array}{r}\text { WORK } \\
\text { ORDER }\end{array}$ & $\begin{array}{c}\text { WO } \\
\text { COMPLETED }\end{array}$ & $\begin{array}{r}\text { LABOR } \\
\text { COST }\end{array}$ & $\begin{array}{r}\text { PARTS } \\
\text { COST }\end{array}$ & $\begin{array}{l}\text { OUTS IDE } \\
\text { CosT }\end{array}$ & $\begin{array}{l}\text { TOTAL } \\
\text { COST }\end{array}$ \\
\hline DIAGNOSTIC MECHANICL & 263658 & $0000 / 00 / 00$ & 129.00 & .00 & .00 & 129.00 \\
\hline REPAIR TYPE TOTAL & & & 129.00 & .00 & .00 & 129.00 \\
\hline REPAIR SUB GROUP TOTAL & & & 129.00 & .00 & .00 & 129.00 \\
\hline REPAIR GROUP TOTAL & & & 129.00 & .00 & .00 & 129.00 \\
\hline PM/MINUS PM TOTAL & & & 129.00 & .00 & .00 & 129.00 \\
\hline ENDYR TOTAL & & & 129.00 & .00 & .00 & 129.00 \\
\hline $\begin{array}{l}\text { 0215L MIRROR, EXTERNAL } \\
\text { REPAIR TYPE TOTAL }\end{array}$ & 237464 & $1993 / 12 / 06$ & $\begin{array}{l}21.50 \\
21.50\end{array}$ & $\begin{array}{l}.00 \\
.00\end{array}$ & $\begin{array}{l}.00 \\
.00\end{array}$ & $\begin{array}{l}21.50 \\
21.50\end{array}$ \\
\hline REPAIR SUB GROUP TOTAL & & & 21.50 & .00 & .00 & 21.50 \\
\hline REPAIR GROUP TOTAL & & & 21.50 & .00 & .00 & 21.50 \\
\hline 13610 AIR SUPPLY LINES & 236515 & $1993 / 10 / 29$ & 419.25 & .00 & .00 & 419.25 \\
\hline $13610 \quad A 18$ SUPPLY LINES & 237426 & $1993 / 12 / 03$ & 150.50 & 1.34 & .00 & 151.84 \\
\hline REPAIR TYPE TOTAL & & & 569.75 & 1.34 & .00 & 571.09 \\
\hline REPAIR SUB GROUP TOTAL & & & 569.75 & 1.34 & .00 & 571.09 \\
\hline 1800R WIEEL/RIM & 236706 & $1993 / 11 / 17$ & .00 & .00 & 347.21 & 347.21 \\
\hline REPAIR TYPE TOTAL & & & .00 & .00 & 347.21 & 347.21 \\
\hline REPAIR SUB GROUP TOTAL & & & .00 & .00 & 347.21 & 347.21 \\
\hline REPAIR GROUP TOTAL & & & 569.75 & 1.34 & 347.21 & 918.30 \\
\hline 4400L FUEL SYSTEM & 237279 & $1993 / 12 / 03$ & 53.75 & .00 & .00 & 53.75 \\
\hline 4400I FUEL SYSTEM & 237542 & $1993 / 12 / 09$ & .00 & .00 & .00 & .00 \\
\hline REPAIR TYPE TOTAL & & & 53.75 & .00 & .00 & 53.75 \\
\hline $\begin{array}{l}\text { 4410R FILTER, PRIMARY } \\
\text { REPAIR TYPE TOTAL, }\end{array}$ & 237319 & $1993 / 12 / 03$ & .00 & .00 & $\begin{array}{l}183.63 \\
183.63\end{array}$ & $\begin{array}{l}183.63 \\
183.63\end{array}$ \\
\hline FUEL LINES & 237319 & $1993 / 12 / 03$ & 301.00 & .00 & .00 & 301.00 \\
\hline$\$ 4150$ FUEL LINES & 237348 & $1993 / 12 / 03$ & 225.75 & .81 & .00 & 226.56 \\
\hline REPAIR TYPE TOTAL & & & 526.75 & .81 & .00 & 527.56 \\
\hline REPAIR SUB GROUP TOTAL & & & 560.50 & .81 & 183.63 & 764.94 \\
\hline ENGINE & 237426 & $1993 / 12 / 03$ & 43.00 & .00 & .00 & 43.00 \\
\hline REPAIR TYPE TOTAL & & & 43.00 & .00 & .00 & 43.00 \\
\hline REPAIR SUB GROUP TOTAL & & & 43.00 & .00 & .00 & 43.00 \\
\hline REPAIR GROUP TOTAL & & & 623.50 & .81 & 183.63 & 807.94 \\
\hline
\end{tabular}


VEHICLE 3220

INT'

ETHANOL TANDEM AXLE

\begin{abstract}
REPAIR
CODE

DESCRIPTION
\end{abstract}

UNIT TOTAL. $\begin{array}{cc}\text { WORK } & \text { WO } \\ \text { ORDER } & \text { COMPLETED }\end{array}$
LABOR
COST

$17,443.19$

PART
COS
3,041

OUTSIDE

$\cos T$

$4,943.73$

$25,420.61$ 
VEHICLE 3220 INT' EXPBASE TANDEM AXLE

\begin{tabular}{|c|c|c|c|c|c|c|}
\hline $\begin{array}{l}\text { REPAIR } \\
\text { CODE }\end{array}$ & $\begin{array}{l}\text { WORK } \\
\text { ORDER }\end{array}$ & $\begin{array}{c}\text { พO } \\
\text { COMPLETED }\end{array}$ & $\begin{array}{r}\text { LABOR } \\
\text { COST }\end{array}$ & $\begin{array}{l}\text { PARTS } \\
\text { COST }\end{array}$ & $\begin{array}{l}\text { OUTSIDE } \\
\text { COST }\end{array}$ & $\begin{array}{l}\text { TOTAL } \\
\text { COST }\end{array}$ \\
\hline SP EQUIP ACCESSORIES & 263446 & $1996 / 09 / 19$ & 10.75 & .00 & .00 & 10.75 \\
\hline REPAIR TYPE TOTAL & & & 32.25 & .00 & .00 & 32.25 \\
\hline REPALR SUB GROUP TOTAL & & & 32.25 & .00 & .00 & 32.25 \\
\hline REPAIR GROUP TOTAL & & & 32.25 & .00 & .00 & 32.25 \\
\hline HYD LINE, PRESSURE & 258825 & $1996 / 03 / 11$ & 279.50 & 52.19 & .00 & 331.69 \\
\hline 73010 HYD LINE, PRESSURE & 258898 & $1996 / 03 / 14$ & 43.00 & .00 & .00 & 43.00 \\
\hline 73010 HYD LINE， PRESSURE & 259226 & $1996 / 03 / 29$ & 86.00 & 29.56 & .00 & 115.56 \\
\hline REPAIR TYPE TOTAL & & & 408.50 & 81.75 & .00 & 490.25 \\
\hline 73100 HYD LINE， RETURN & 259015 & $1996 / 03 / 18$ & 75.25 & .00 & .00 & 75.25 \\
\hline REPAIR TYPE TOTAL & & & 75.25 & .00 & .00 & 75.25 \\
\hline REPAIR SUB GROUP TOTAL & & & 483.75 & 81.75 & .00 & 565.50 \\
\hline $\begin{array}{l}\text { 7721R HYD TANK FILTER } \\
\text { REPAIR TYPE TOTAL }\end{array}$ & 262383 & $1996 / 09 / 20$ & $\begin{array}{l}21.50 \\
21.50\end{array}$ & $\begin{array}{l}6.49 \\
6.49\end{array}$ & $\begin{array}{l}.00 \\
.00\end{array}$ & $\begin{array}{l}27.99 \\
27.99\end{array}$ \\
\hline 7725L HYDRAULIC FLUID & 259020 & $1996 / 03 / 18$ & 43.00 & 17.76 & .00 & 60.76 \\
\hline REPAIR TYPE TOTAL & & & 43.00 & 17.76 & .00 & 60.76 \\
\hline REPAIR SUB GROUP TOTAL & & & 64.50 & 24.25 & .00 & 88.75 \\
\hline REPAIR GROUP TOTAL & & & 548.25 & 106.00 & .00 & 654.25 \\
\hline AUX AIR CYLINDER & 262734 & $1996 / 08 / 26$ & 21.50 & .00 & .00 & 21.50 \\
\hline REPAIR TYPE TOTAL & & & 21.50 & .00 & .00 & 21.50 \\
\hline REPAIR SUB GROUP TOTAL & & & 22.50 & .00 & .00 & 21.50 \\
\hline GENERAL GREASING & M08223 & $1996 / 02 / 08$ & 86.00 & .88 & .00 & 86.88 \\
\hline GENERAL GREASING & M08408 & $1996 / 03 / 14$ & 86.00 & .88 & .00 & 86.88 \\
\hline 98200 GENERAL GREASING & M09047 & $1996 / 08 / 15$ & 86.00 & .88 & .00 & 86.88 \\
\hline 98200 GENERAL GREASING & M09176 & $1996 / 09 / 20$ & 86.00 & .88 & .00 & 86.88 \\
\hline 98200 GENERAL GREASING & M09297 & $1996 / 10 / 28$ & 86.00 & .88 & .00 & 86.88 \\
\hline REPAIR TYPE TOTAL & & & 430.00 & 4.40 & .00 & 434.40 \\
\hline TRUCK INSPECTION & 262383 & $1996 / 09 / 20$ & 43.00 & .00 & .00 & 43.00 \\
\hline REPAIR TYPE TOTAL & & & 43.00 & .00 & .00 & 43.00 \\
\hline REPAIR SUB GROUP TOTAL & & & 473.00 & 4.40 & .00 & 477.40 \\
\hline REPAIR GROUP TOTAL & & & 494.50 & 4.40 & .00 & 498.90 \\
\hline PM/MINUS PM TOTAL & & & $5,657.94$ & $1,538.77$ & 864.65 & $8,061.36$ \\
\hline ENDYR TOTAL & & & 5.829 .94 & $1,549.75$ & 890.39 & $8,270.08$ \\
\hline
\end{tabular}


VEHICLE 3220 INT' EXPBASE TANDEM AXLE

\begin{tabular}{|c|c|c|c|c|c|c|}
\hline $\begin{array}{l}\text { REPAIR } \\
\text { COINE }\end{array}$ & $\begin{array}{l}\text { WORK } \\
\text { ORDER }\end{array}$ & $\begin{array}{c}\text { พo } \\
\text { COMPLETED }\end{array}$ & $\begin{array}{r}\text { LABOR } \\
\text { COST }\end{array}$ & $\begin{array}{r}\text { PARTS } \\
\text { COST }\end{array}$ & $\begin{array}{l}\text { OUTS IDE } \\
\text { COST }\end{array}$ & $\begin{array}{l}\text { TOTAL } \\
\text { COST }\end{array}$ \\
\hline $\begin{array}{l}4200 \mathrm{~T} \text { COOLING SYSTEM } \\
\text { REPAIR TYPE TOTAL }\end{array}$ & 254342 & $1996 / 11 / 05$ & $\begin{array}{l}43.00 \\
43.00\end{array}$ & $\begin{array}{l}.00 \\
.00\end{array}$ & $\begin{array}{l}.00 \\
.00\end{array}$ & $\begin{array}{l}43.00 \\
43.00\end{array}$ \\
\hline 4212R COOLANT FILTER & 262383 & $1996 / 09 / 20$ & 21.50 & 8.88 & .00 & 30.38 \\
\hline REPAIR TYPE TOTAL & & & 21.50 & 8.88 & .00 & 30.38 \\
\hline FAN CLUTCH & 263529 & $1996 / 10 / 31$ & 311.75 & .00 & .00 & 311.75 \\
\hline 42260 FAN CLUTCH & 263529 & $1996 / 10 / 31$ & 236.50 & 1.64 & 567.58 & 805.72 \\
\hline 4226I FAN CLUTCH & 263529 & $1996 / 10 / 31$ & 64.50 & .00 & .00 & 64.50 \\
\hline REPAIR TYPE TOTAL & & & 612.75 & 1.64 & 567.58 & $1,181.97$ \\
\hline $\begin{array}{l}\text { 4231L WATER PUMP BELT } \\
\text { REPAIR TYPE TOTAL }\end{array}$ & 262383 & $1996 / 09 / 20$ & $\begin{array}{l}21.50 \\
21.50\end{array}$ & $\begin{array}{l}.00 \\
.00\end{array}$ & $\begin{array}{l}.00 \\
.00\end{array}$ & $\begin{array}{l}21.50 \\
21.50\end{array}$ \\
\hline REPAIR SUB GROUP TOTAL & & & 698.75 & 10.52 & 567.58 & $1,276.85$ \\
\hline 4410R FILTER, PRIMARY & 259316 & $1996 / 03 / 29$ & 118.25 & .00 & .00 & 118.25 \\
\hline 4410R FILTER, PRIMARY & 262383 & $1996 / 09 / 20$ & .00 & .00 & 8.22 & 8.22 \\
\hline REPAIR TYPE TOTAL & & & 118.25 & .00 & 8.22 & 126.47 \\
\hline 4411R FILTER， SECONDARY & 262383 & $1996 / 09 / 20$ & .00 & .00 & 8.22 & 8.22 \\
\hline REPAIR TYPE TOTAL & & & .00 & .00 & 8.22 & 8.22 \\
\hline REPAIR SUB GROUP TOTAL & & & 118.25 & .00 & 16.44 & 134.69 \\
\hline REPAIR GROUP TOTAL & & & 817.00 & 10.52 & 584.02 & 1.411 .54 \\
\hline 51000 ACCESSORIES, GENERAL & 258681 & $1996 / 03 / 18$ & 408.50 & 3.09 & .00 & 411.59 \\
\hline REPAIR TYPE TOTAL & & & 408.50 & 3.09 & .00 & 411.59 \\
\hline 51100 TARPALIN ASSEMBLY & 261055 & $1996 / 06 / 14$ & 215.00 & .84 & .00 & 215.84 \\
\hline REPAIR TYPE TOTAL & & & 215.00 & .84 & .00 & 215.84 \\
\hline REPAIR SUB GROUP TOTAL & & & 623.50 & 3.93 & .00 & 627.43 \\
\hline 52400 BOX SHAKER & 258610 & $1996 / 02 / 29$ & 86.00 & 105.00 & .00 & 191.00 \\
\hline 52400 BOX SHAKER & 260747 & $1996 / 05 / 30$ & 161.25 & 36.27 & .00 & 197.52 \\
\hline REPAIR TYPE TOTAL & & & 247.25 & 141.27 & .00 & 388.52 \\
\hline REPAIR SUB GROUP TOTAL & & & 247.25 & 141.27 & .00 & 388.52 \\
\hline 54100 SNOW PLOW FRAME & 256942 & $1996 / 01 / 10$ & 43.00 & .00 & .00 & 43.00 \\
\hline REPAIR TYPE TOTAI. & & & 43.00 & .00 & .00 & 43.00 \\
\hline REPMIR SUB GROUP TOTAL & & & 13.00 & .00 & .00 & 43.00 \\
\hline REPAIR GROUP TOTAL & & & 913.75 & 145.20 & .00 & $1,058,95$ \\
\hline 6600L SP EQUIP ACCESSORIES & 262522 & $1996 / 08 / 15$ & 21.50 & .00 & .00 & 21.50 \\
\hline
\end{tabular}


VEHICLE 3220 INT' EXPBASE TANDEM NXLE

\begin{tabular}{|c|c|c|c|c|c|c|}
\hline $\begin{array}{l}\text { REPAIR } \\
\text { CODE }\end{array}$ & $\begin{array}{l}\text { WORK } \\
\text { ORDER }\end{array}$ & $\begin{array}{c}\text { พO } \\
\text { COMPLETED }\end{array}$ & $\begin{array}{r}\text { LABOR } \\
\text { COST }\end{array}$ & $\begin{array}{l}\text { PARTS } \\
\text { COST }\end{array}$ & $\begin{array}{l}\text { OUTSIDE } \\
\text { COST }\end{array}$ & $\begin{array}{l}\text { TOTAL } \\
\text { Cost }\end{array}$ \\
\hline 3107R ALTERNATOR BELT & 263660 & $1996 / 10 / 09$ & 107.50 & .00 & 10.56 & 118.06 \\
\hline REPAIR TYPE TOTAL & & & 107.50 & .00 & 10.56 & 118.06 \\
\hline 31200 WIRES & 260975 & $1996 / 06 / 07$ & 53.75 & .00 & .00 & 53.75 \\
\hline REPAIR TYPE TOTAL & & & 53.75 & .00 & .00 & 53.75 \\
\hline REPAIR SUB GROUP TOTAL & & & 182.75 & .00 & 10.56 & 193.31 \\
\hline BATTERY & 263916 & $1996 / 10 / 21$ & 75.25 & 346.08 & .00 & 421.33 \\
\hline REPAIR TYPE TOTAL & & & 75.25 & 346.08 & .00 & 421.33 \\
\hline REPAIR SUB GROUP TOTAL & & & 75.25 & 346.08 & .00 & 421.33 \\
\hline $\begin{array}{l}34000 \text { LIGHTING SYSTEM } \\
\text { REPAIR TYPE TOTAL }\end{array}$ & 261426 & $1996 / 07 / 02$ & $\begin{array}{l}107.50 \\
107.50\end{array}$ & $\begin{array}{l}13.00 \\
13.00\end{array}$ & $\begin{array}{l}.00 \\
.00\end{array}$ & $\begin{array}{l}120.50 \\
120.50\end{array}$ \\
\hline HEADLAMP & 256887 & $1996 / 01 / 05$ & 10.75 & 12.36 & .00 & 23.11 \\
\hline HEADLAMP & 256942 & $1996 / 01 / 10$ & 21.50 & 12.36 & .00 & 33.86 \\
\hline REPAIR TYPE TOTAL & & & 32.25 & 24.72 & .00 & 56.97 \\
\hline STOPLAMP & 259084 & $1996 / 03 / 20$ & 64.50 & .00 & .00 & 64.50 \\
\hline 34100 STOPLAMP & 262855 & $1996 / 09 / 03$ & 43.00 & .00 & .00 & 43.00 \\
\hline REPAIR TYPE TOTAL. & & & 107.50 & .00 & .00 & 107.50 \\
\hline TURNSIGNAL LAAMP & 262855 & $1996 / 09 / 03$ & 107.50 & 12.97 & .00 & 120.47 \\
\hline 34150 TURNSIGNAL LAMP & 262860 & $1996 / 09 / 10$ & .00 & 1.76 & .00 & 1.76 \\
\hline REPAIR TYPE TOTAL & & & 107.50 & 14.73 & .00 & 122.23 \\
\hline 34300 CLEARANCE/MARKER IMP & 258560 & $1996 / 02 / 29$ & 43.00 & .00 & .00 & 43.00 \\
\hline CLEARANCE/MARKER LMP & 259431 & $1996 / 04 / 03$ & 21.50 & 1.83 & .00 & 23.33 \\
\hline 34300 CLEARANCE/MARKER LMP & 261107 & $1996 / 06 / 14$ & 43.00 & 1.82 & .00 & 44.82 \\
\hline REPAIR TYPE TOTAL & & & 107.50 & 3.65 & .00 & 111.15 \\
\hline 34310 INTERIOR/DASH LAMP & 259046 & $1996 / 03 / 20$ & 21.50 & .80 & .00 & 22.30 \\
\hline 34310 INTERIOR/DASH LAMP & 260283 & $1996 / 06 / 07$ & 21.50 & .93 & .00 & 22.43 \\
\hline REPAIR TYPE TOTAL & & & 43.00 & 1.73 & .00 & $\mathbf{4 4 . 7 3}$ \\
\hline 34400 STROBE LAMP & 257464 & $1996 / 01 / 23$ & 43.00 & .00 & .00 & 43.00 \\
\hline REPAIR TYPE TOTAL & & & 43.00 & .00 & .00 & 43.00 \\
\hline 34500 SANDER/WORK LAMP & 264574 & $1996 / 11 / 08$ & 53.75 & 9.68 & .00 & 63.43 \\
\hline REQAIR TYQE TOTAL & & & 53.75 & 9.68 & .00 & 63.43 \\
\hline 3455R LAMP SWITCH(S) & 259084 & $1996 / 03 / 20$ & .00 & .00 & 9.81 & 9.81 \\
\hline REPAI" TYPE TOTAL & & & .00 & .00 & 9.81 & 9.81 \\
\hline REPAIR ::ITB GROUP TOTAL & & & 602.00 & 67.51 & 9.81 & 679.32 \\
\hline RERAIR GROUP TOTAL & & & 860.00 & 413.59 & 20.37 & $1,293.96$ \\
\hline
\end{tabular}


VEHICLE 3220 INT' EXPBASE TANDEM AXLE

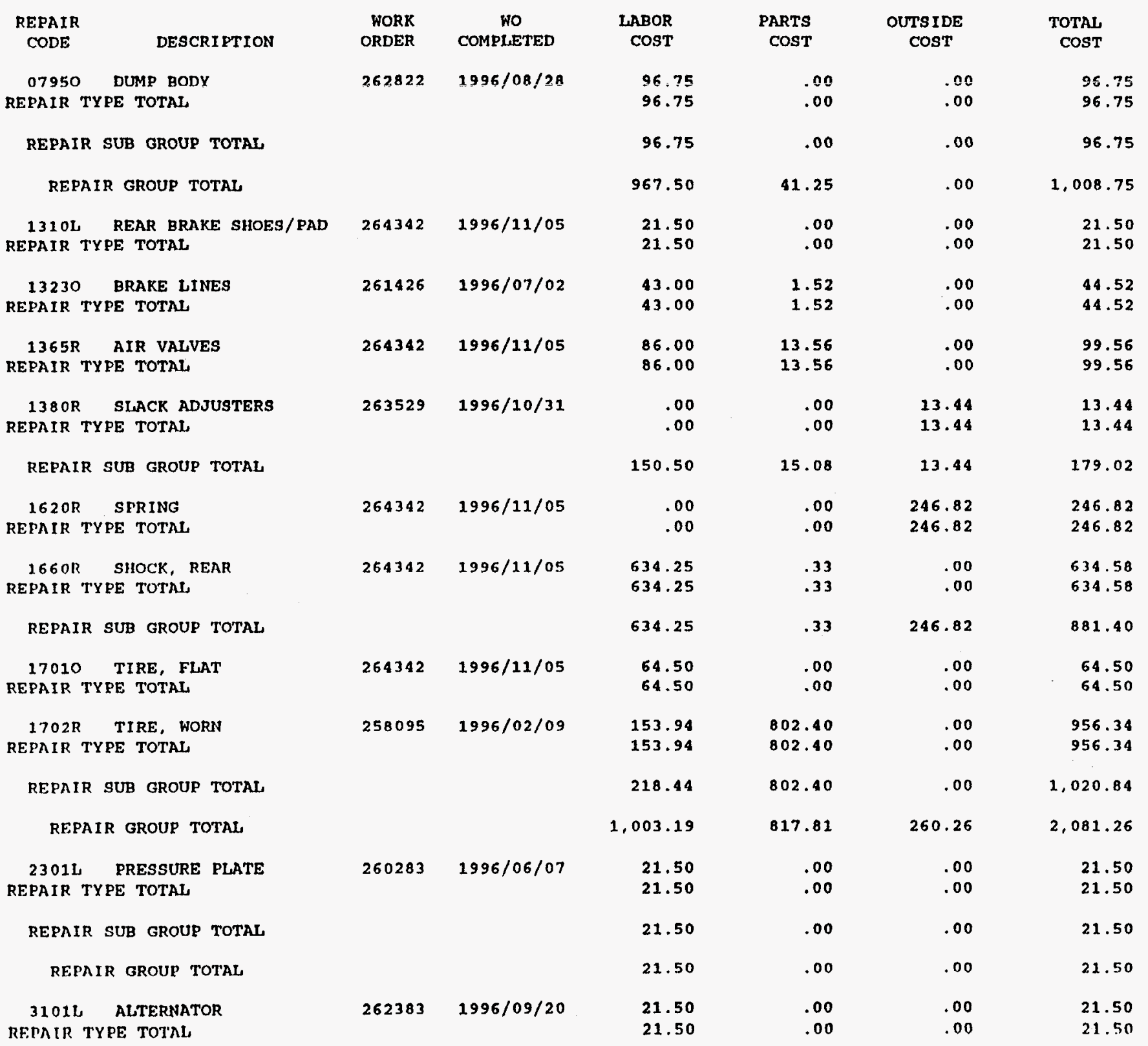


VEHICLE 3220 INT' EXPBASE TANDEM AXLE

\begin{tabular}{|c|c|c|c|c|c|c|}
\hline $\begin{array}{l}\text { REPAIR } \\
\text { CODE }\end{array}$ & $\begin{array}{l}\text { WORK } \\
\text { ORDER }\end{array}$ & $\begin{array}{c}\text { WO } \\
\text { COMPLETED }\end{array}$ & $\begin{array}{r}\text { LABOR } \\
\text { COST }\end{array}$ & $\begin{array}{r}\text { PARTS } \\
\text { COST }\end{array}$ & $\begin{array}{l}\text { OUTSIDE } \\
\text { COST }\end{array}$ & $\begin{array}{l}\text { TOTAL } \\
\text { COST }\end{array}$ \\
\hline $\begin{array}{l}9895 \mathrm{I} \text { DIAGNOSTIC MECHANICL } \\
\text { REPAIR TYPE TOTAL }\end{array}$ & 251266 & $1995 / 06 / 12$ & $\begin{array}{l}43.00 \\
43.00\end{array}$ & $\begin{array}{l}.00 \\
.00\end{array}$ & $\begin{array}{l}.00 \\
.00\end{array}$ & $\begin{array}{l}43.00 \\
43.00\end{array}$ \\
\hline REPAIR SUB GROUP TOTAL & & & 688.00 & 6.13 & 10.86 & 704.99 \\
\hline REPAIR GROUP TOTAL & & & 688.00 & 6.13 & 10.86 & 704.99 \\
\hline PM/MINUS PM TOTAL & & & $3,375.50$ & 959.74 & $1,099.65$ & $5,434.89$ \\
\hline ENDYR TOTAL & & & 3.687 .25 & 980.82 & $1,168.03$ & $5,836.10$ \\
\hline $\begin{array}{l}\text { 1110R WET TYPE HUB ASSEMB. } \\
\text { REPAIR TYPE TOTAL }\end{array}$ & 263529 & $1996 / 10 / 31$ & $\begin{array}{l}21.50 \\
21.50\end{array}$ & $\begin{array}{l}.00 \\
.00\end{array}$ & .00 & $\begin{array}{l}21.50 \\
21.50\end{array}$ \\
\hline $\begin{array}{l}\text { 1115L PMA } \\
\text { REPAIR TYPE TOTAL }\end{array}$ & 260753 & $1996 / 05 / 30$ & $\begin{array}{l}129.00 \\
129.00\end{array}$ & $\begin{array}{l}10.98 \\
10.98\end{array}$ & $\begin{array}{l}25.74 \\
25.74\end{array}$ & $\begin{array}{l}165.72 \\
165.72\end{array}$ \\
\hline $\begin{array}{l}1120 \mathrm{~L} \text { PMB } \\
\text { REPAIR TYPE TOTAL }\end{array}$ & 262383 & $1996 / 09 / 20$ & $\begin{array}{l}21.50 \\
21.50\end{array}$ & $\begin{array}{l}.00 \\
.00\end{array}$ & $\begin{array}{l}.00 \\
.00\end{array}$ & $\begin{array}{l}21.50 \\
21.50\end{array}$ \\
\hline REPAIR SUB GROUP TOTAL & & & 172.00 & 10.98 & 25.74 & 208.72 \\
\hline REPAIR GROUP TOTAL & & & 172.00 & 10.98 & 25.74 & 208.72 \\
\hline PM/MINUS PM TOTAL & & & 172.00 & 10.98 & 25.74 & 208.72 \\
\hline 0215R MIRROR， EXTERNAL & 257464 & $1996 / 01 / 23$ & 64.50 & 13.81 & .00 & 78.31 \\
\hline MIRROR, EXTERNAL & 257648 & $1996 / 01 / 30$ & 10.75 & 13.81 & .00 & 24.56 \\
\hline 02150 MIRROR, EXTERNAL & 261497 & $1996 / 07 / 03$ & 53.75 & .82 & .00 & 54.57 \\
\hline $\begin{array}{l}\text { 0215I MIRROR, EXTERNAL } \\
\text { REPAIR TYPE TOTAL }\end{array}$ & 261865 & $1996 / 07 / 23$ & $\begin{array}{l}107.50 \\
236.50\end{array}$ & $\begin{array}{r}.00 \\
20.44\end{array}$ & $\begin{array}{l}.00 \\
.00\end{array}$ & $\begin{array}{l}107.50 \\
264.94\end{array}$ \\
\hline $\begin{array}{l}\text { 0217R MIRROR, EXT CONVEX } \\
\text { REPAIR TYPE TOTAL }\end{array}$ & 261603 & $1996 / 07 / 03$ & $\begin{array}{l}32.25 \\
32.25\end{array}$ & $\begin{array}{l}12.81 \\
12.81\end{array}$ & $\begin{array}{l}.00 \\
.00\end{array}$ & $\begin{array}{l}45.06 \\
45.06\end{array}$ \\
\hline REPAIR SUB GROUP TOTAL. & & & 268.75 & 41.25 & .00 & 310.00 \\
\hline $\begin{array}{l}03000 \text { GAUGES/INSTRUMENTS } \\
\text { REPAIR TYPE TOTAL }\end{array}$ & 260283 & $1996 / 06 / 07$ & $\begin{array}{l}236.50 \\
236.50\end{array}$ & $\begin{array}{l}.00 \\
.00\end{array}$ & $\begin{array}{l}.00 \\
.00\end{array}$ & $\begin{array}{l}236.50 \\
236.50\end{array}$ \\
\hline 03400 SPEEDOMETER & 259020 & $1996 / 03 / 18$ & 86.00 & .00 & .00 & 86.00 \\
\hline 03400 SPEEDOMETER & 259046 & $1996 / 03 / 20$ & 139.75 & .00 & .00 & 139.75 \\
\hline REPAIR TYPE TOTAL & & & 225.75 & .00 & .00 & 225.75 \\
\hline TACHOMETER & 259020 & $1996 / 03 / 18$ & 96.75 & .00 & .00 & 96.75 \\
\hline 03500 TACHOMETER & 259046 & $1996 / 03 / 20$ & 43.00 & .00 & .00 & 43.00 \\
\hline REPAIR TYPE TOTAL & & & 139.75 & .00 & .00 & 139.75 \\
\hline REEAIR SUB GROUP TOTAL & & & 602.00 & .00 & .00 & 602.00 \\
\hline
\end{tabular}


VEUICLE 3220 INT' EXPBASE TANDEM NXLE

\begin{tabular}{|c|c|c|c|c|c|c|}
\hline $\begin{array}{l}\text { REPAIR } \\
\text { CODE }\end{array}$ & $\begin{array}{l}\text { WORK } \\
\text { ORDER }\end{array}$ & $\begin{array}{c}\text { WO } \\
\text { COMPLETED }\end{array}$ & $\begin{array}{r}\text { LABORR } \\
\text { COST }\end{array}$ & $\begin{array}{r}\text { PARTS } \\
\text { COST }\end{array}$ & $\begin{array}{l}\text { OUTS IDE } \\
\text { COST }\end{array}$ & $\begin{array}{r}\text { TOTAL } \\
\text { COST }\end{array}$ \\
\hline 52400 goX sunker & 252989 & $1995 / 07 / 26$ & 107.50 & $\mathbf{7} . \hat{\mathbf{9}} \mathbf{3}$ & .00 & 114.93 \\
\hline REPAIR TYPE TOTAL & & & 107.50 & 7.43 & .00 & 114.93 \\
\hline 52440 BOX SPRAY PUMP & 242973 & $1995 / 02 / 02$ & 86.00 & 8.75 & .00 & 94.75 \\
\hline REPAIR TYPE TOTAL & & & 86.00 & B. 75 & .00 & 94.75 \\
\hline REPAIR SUB GROUP TOTAL & & & 193.50 & 16.18 & .00 & 209.68 \\
\hline REPAIR GROUP TOTAL & & & 440.75 & 16.18 & 77.43 & 534.36 \\
\hline 73010 HYD LINE, PRESSURE & 251190 & $1995 / 05 / 03$ & 86.00 & 1.42 & .00 & 87.42 \\
\hline REPAIR TYPE TOTAL & & & 86.00 & 1.42 & .00 & 87.42 \\
\hline REPAIR SUB GROUP TOTAL & & & 86.00 & 1.42 & .00 & 87.42 \\
\hline $7400 \mathrm{I}$ HYDRAULIC VALVES & 255966 & $1995 / 11 / 30$ & 75.25 & .00 & .00 & 75.25 \\
\hline REPAIR TYPE TOTAL & & & 75.25 & .00 & .00 & 75.25 \\
\hline 74010 HYDRACTLIC VALVE & 256084 & $1995 / 12 / 07$ & 64.50 & .00 & .00 & 64.50 \\
\hline REPAIR TYPE TOTAL & & & 64.50 & .00 & .00 & 64.50 \\
\hline REPAIR SUB GROUP TOTAL & & & 139.75 & .00 & .00 & 139.75 \\
\hline 76010 QUICK DISCONNECT & 242973 & $1995 / 02 / 02$ & 21.50 & 3.24 & .00 & 24.74 \\
\hline REPNIR TYPE TOTAL & & & 21.50 & 3.24 & .00 & 24.74 \\
\hline REPAIR SUB GROUP TOTAL & & & 21.50 & 3.24 & .00 & 24.74 \\
\hline 7721R HYD TANK FILTER & 253215 & $1995 / 08 / 21$ & 10.75 & 6.49 & .00 & 17.24 \\
\hline REPAIR TYPE TOTAL & & & 10.75 & 6.49 & .00 & 17.24 \\
\hline 7725L HYDRAULIC FLUID & 251190 & $1995 / 05 / 03$ & .00 & 29.60 & .00 & 29.60 \\
\hline 77251 HYDRAULIC FLUID & 255966 & $1995 / 11 / 30$ & .00 & 44.40 & .00 & 44.40 \\
\hline REPAIR TYPE TOTAL & & & .00 & 74.00 & .00 & 74.00 \\
\hline REPAIR SUB GROUP TOTAL & & & 10.75 & 80.49 & .00 & 91.24 \\
\hline REPAIR GROUP TOTAL & & & 258.00 & 85.15 & .00 & 343.15 \\
\hline 98200 GENERAL GREASING & M06599 & $1995 / 01 / 19$ & 86.00 & .87 & .00 & 86.87 \\
\hline 98200 GENERAL GREASINO & M06920 & $1995 / 03 / 15$ & 107.50 & .87 & 4.92 & 113.29 \\
\hline GENERAL GREASING & M07085 & $1995 / 04 / 26$ & 86.00 & .87 & .00 & 86.87 \\
\hline GENERAL GREASING & $M 07393$ & $1995 / 07 / 21$ & 86.00 & .88 & .00 & 86.88 \\
\hline 98200 GENERAL GREASING & M07547 & $1995 / 08 / 24$ & 86.00 & .88 & 5.94 & 92.82 \\
\hline GENERAL GREASING & M07633 & $1995 / 09 / 18$ & 86.00 & .88 & .00 & 86.88 \\
\hline 98200 GENERAL GREASING & M07963 & $1995 / 12 / 07$ & 86.00 & .88 & .00 & 86.88 \\
\hline REPAIR TYPE TOTMI, & & & 623.50 & 6.13 & 10.85 & 610.49 \\
\hline 9860I TRUCK INSPECTION & 253215 & $1995 / 08 / 21$ & 21.50 & .00 & .00 & 21.50 \\
\hline REPAIR TYPE TOTAL & & & 21.50 & .00 & .00 & 21.50 \\
\hline
\end{tabular}

REPAIR TYPE TOTAL 
VEHICLE 3220 INT' EXPBASE TANDEM AXLE

\begin{tabular}{|c|c|c|c|c|c|c|}
\hline $\begin{array}{l}\text { REPAIR } \\
\text { CODE }\end{array}$ & $\begin{array}{l}\text { WORK } \\
\text { ORDER }\end{array}$ & $\begin{array}{c}\text { พO } \\
\text { COMPLETED }\end{array}$ & $\begin{array}{l}\text { LABOR } \\
\text { COST }\end{array}$ & $\begin{aligned} \text { PARTS } \\
\text { COST }\end{aligned}$ & $\begin{array}{l}\text { outsIDE } \\
\text { COST }\end{array}$ & $\begin{array}{l}\text { TOTAL } \\
\text { COST }\end{array}$ \\
\hline SANDER/WORK LAMP & 249176 & $1995 / 02 / 14$ & 10.75 & .00 & .00 & 10.75 \\
\hline REPAIR TYPE TOTAL & & & 10.75 & .00 & .00 & 10.75 \\
\hline REPAIR SUB GROUP TOTAL & & & 698.75 & 46.29 & .00 & 745.04 \\
\hline REPAIR GROUP TOTAL & & & 698.75 & 46.29 & .00 & 745.04 \\
\hline $\begin{array}{l}\text { 4200I COOLING SYSTEM } \\
\text { REPAIR TYPE TOTAL }\end{array}$ & 248571 & $1995 / 01 / 25$ & $\begin{array}{l}64.50 \\
64.50\end{array}$ & $\begin{array}{l}.00 \\
.00\end{array}$ & $\begin{array}{l}.00 \\
.00\end{array}$ & $\begin{array}{l}64.50 \\
64.50\end{array}$ \\
\hline $\begin{array}{l}\text { 4212R COOLANT FILTER } \\
\text { REDAIR TYPE TOTAL }\end{array}$ & 253215 & $1995 / 08 / 21$ & $\begin{array}{l}10.75 \\
10.75\end{array}$ & $\begin{array}{l}8.88 \\
8.88\end{array}$ & $\begin{array}{l}.00 \\
.00\end{array}$ & $\begin{array}{l}19.63 \\
19.63\end{array}$ \\
\hline $\begin{array}{l}\text { 4226R FAN CLUTCH } \\
\text { REPAIR TYPE TOTAL }\end{array}$ & 250685 & $1995 / 06 / 12$ & $\begin{array}{l}86.00 \\
86.00\end{array}$ & $\begin{array}{l}.66 \\
.66\end{array}$ & $\begin{array}{l}753.93 \\
753.93\end{array}$ & $\begin{array}{l}840.59 \\
840.59\end{array}$ \\
\hline REPAIR SUB GROUP TOTAL & & & 161.25 & 9.54 & 753.93 & 924.72 \\
\hline CLAMPS & 254394 & $1995 / 09 / 22$ & 21.50 & .00 & .00 & 21.50 \\
\hline REPAIR TYPE TOTAL & & & 21.50 & .00 & .00 & 21.50 \\
\hline REPAIR SUB GROUP TOTAL & & & 21.50 & .00 & .00 & 21.50 \\
\hline $\begin{array}{l}\text { 4410R FILTER, PRIMARY } \\
\text { REPAIR TYPE TOTAL }\end{array}$ & 253215 & $1995 / 08 / 21$ & $\begin{array}{l}10.75 \\
10.75\end{array}$ & $\begin{array}{l}.00 \\
.00\end{array}$ & $\begin{array}{l}7.72 \\
7.72\end{array}$ & $\begin{array}{l}18.47 \\
18.47\end{array}$ \\
\hline $\begin{array}{l}\text { 4411R FILTER, SECONDARY } \\
\text { REPAIR TYPE TOTAL }\end{array}$ & 253215 & $1995 / 08 / 21$ & $\begin{array}{l}10.75 \\
10.75\end{array}$ & $\begin{array}{l}.00 \\
.00\end{array}$ & $\begin{array}{l}7.72 \\
7.72\end{array}$ & $\begin{array}{l}18.47 \\
18.47\end{array}$ \\
\hline REPAIR SUB GROUP TOTAL & & & 21.50 & .00 & 15.44 & 36.94 \\
\hline REPAIR GROUP TOTAL & & & 204.25 & 9.54 & 769.37 & 983.16 \\
\hline $\begin{array}{l}51000 \text { ACCESSORIES, GENERAL } \\
\text { REPAIR TYPE TOTAL }\end{array}$ & 254427 & $1995 / 09 / 26$ & $\begin{array}{l}.00 \\
.00\end{array}$ & $\begin{array}{l}.00 \\
.00\end{array}$ & $\begin{array}{l}58.62 \\
58.62\end{array}$ & $\begin{array}{l}58.62 \\
58.62\end{array}$ \\
\hline MUD FLAP (S) & 242973 & $1995 / 02 / 02$ & 32.25 & .00 & .00 & 32.25 \\
\hline MUD FLAP (S) & 253215 & $1995 / 08 / 21$ & 32.25 & .00 & .00 & 32.25 \\
\hline $5102 R \quad M U D$ FLAP (S) & 256216 & $1995 / 12 / 07$ & 43.00 & .00 & .00 & 43.00 \\
\hline REPAIR TYPE TOTAL & & & 107.50 & .00 & .00 & 107.50 \\
\hline TARPALIN ASSEMBLY & 248131 & $1995 / 01 / 12$ & 75.25 & .00 & .00 & 75.25 \\
\hline 51100 TARPALIN ASSEMBLY & 254427 & $1995 / 09 / 26$ & 21.50 & .00 & .00 & 21.50 \\
\hline REPAIR TYPE TOTAL & & & 96.75 & .00 & .00 & 96.75 \\
\hline $\begin{array}{l}\text { 5115R W/WASHER BLADES } \\
\text { REPAIR TYPE TOTAL }\end{array}$ & 249363 & $1995 / 02 / 27$ & $\begin{array}{l}43.00 \\
43.00\end{array}$ & $\begin{array}{l}.00 \\
.00\end{array}$ & $\begin{array}{l}18.81 \\
18.81\end{array}$ & $\begin{array}{l}61.81 \\
61.81\end{array}$ \\
\hline REPAIR SUB GROUP TOTAL & & & 247.25 & .00 & 77.43 & 324.68 \\
\hline
\end{tabular}


VEHICLE 3220 INT' EXPBASE TANDEM AXIE

\begin{tabular}{|c|c|c|c|c|c|c|}
\hline $\begin{array}{l}\text { REPAIR } \\
\text { CODE }\end{array}$ & $\begin{array}{r}\text { WORR } \\
\text { ORDER }\end{array}$ & $\begin{array}{c}\text { WO } \\
\text { COMPLETED }\end{array}$ & $\begin{array}{r}\text { LABOR } \\
\text { COST }\end{array}$ & $\begin{array}{r}\text { PARTS } \\
\text { COST }\end{array}$ & $\begin{array}{l}\text { OUTSIDE } \\
\text { COST }\end{array}$ & $\begin{array}{l}\text { TOTAL } \\
\text { COST }\end{array}$ \\
\hline $\begin{array}{l}17010 \text { TIRE, FLAT } \\
\text { REPAIR TYPE TOTAL }\end{array}$ & 250001 & $1955 / 03 / 17$ & $\begin{array}{l}64.50 \\
64.50\end{array}$ & $\begin{array}{l}.00 \\
.00\end{array}$ & $\begin{array}{l}.00 \\
.00\end{array}$ & $\begin{array}{l}64.50 \\
64.50\end{array}$ \\
\hline $\begin{array}{l}\text { 1702R TIRE, WORN } \\
\text { REPAIR TYPE TOTAI }\end{array}$ & 253774 & $1995 / 08 / 2 B$ & $\begin{array}{l}75.25 \\
75.25\end{array}$ & $\begin{array}{l}592.84 \\
592.84\end{array}$ & $\begin{array}{l}.00 \\
.00\end{array}$ & $\begin{array}{l}668.09 \\
668.09\end{array}$ \\
\hline REPAIR SUB GROUP TOTAL & & & 139.75 & 592.84 & .00 & 732.59 \\
\hline REPAIR GROUP TOTAL & & & 516.00 & 748.14 & 161.24 & $1,425.38$ \\
\hline PRESSURE PLATE & 253707 & $1995 / 08 / 28$ & 32.25 & .00 & .00 & 32.25 \\
\hline $\begin{array}{l}2301 \mathrm{~L} \text { PRESSURE PLATE } \\
\text { REPAIR TYPE TOTAL }\end{array}$ & 256461 & $2995 / 12 / 15$ & $\begin{array}{l}21.50 \\
53.75\end{array}$ & $\begin{array}{l}.00 \\
.00\end{array}$ & $\begin{array}{l}.00 \\
.00\end{array}$ & $\begin{array}{l}21.50 \\
53.75\end{array}$ \\
\hline REPAIR SUB GROUP TOTAL & & & 53.75 & .00 & .00 & 53.75 \\
\hline $\begin{array}{l}\text { 2791L TRANSMISSION FLUID } \\
\text { REPAIR TYPE TOTAL }\end{array}$ & 255091 & $1995 / 10 / 25$ & $\begin{array}{l}.00 \\
.00\end{array}$ & $\begin{array}{l}14.80 \\
14.80\end{array}$ & $\begin{array}{l}.00 \\
.00\end{array}$ & $\begin{array}{l}14.80 \\
14.80\end{array}$ \\
\hline REPAIR SUB GROUP TOTAL & & & .00 & 14.80 & .00 & 14.80 \\
\hline REPAIR GROUP TOTAL & & & 53.75 & 14.80 & .00 & 68.55 \\
\hline HEADLAMP & 249176 & $1995 / 02 / 14$ & 10.75 & 11.00 & .00 & 21.75 \\
\hline HEADLAMP & 255544 & $1995 / 11 / 14$ & 43.00 & 12.36 & .00 & 55.36 \\
\hline RERAIR TXPE TOTAL & & & 53.75 & 23.36 & .00 & 77.11 \\
\hline TURNS IGNAL LAMP & 250656 & $1995 / 04 / 20$ & 204.25 & .00 & .00 & 2.04 .25 \\
\hline TURNS I GNAL LAMP & 251266 & $1995 / 06 / 12$ & 32.25 & 8.29 & .00 & 40.54 \\
\hline REPAIR TYPE TOTAL & & & 236.50 & 8.29 & .00 & 244.79 \\
\hline $\begin{array}{l}34200 \text { TAIL/PARK LAMP } \\
\text { REPAIR TYPE TOTAL }\end{array}$ & 251266 & $1995 / 06 / 12$ & $\begin{array}{l}.00 \\
.00\end{array}$ & $\begin{array}{l}.00 \\
.00\end{array}$ & $\begin{array}{l}.00 \\
.00\end{array}$ & $\begin{array}{l}.00 \\
.00\end{array}$ \\
\hline CLEARANCE/MARKER LMP & 242973 & $1995 / 02 / 02$ & 86.00 & 2.28 & .00 & 88.28 \\
\hline CLEARANCE/MARKER LMP & 242973 & $1995 / 02 / 02$ & .00 & 10.41 & .00 & 10.41 \\
\hline CLEARANCE/MARKER IMP & 250394 & $1995 / 04 / 04$ & 75.25 & .00 & .00 & 75.25 \\
\hline CLEARANCE/MARKER LMP & 252675 & $1995 / 07 / 10$ & 43.00 & .00 & .00 & 43.00 \\
\hline CLEARANCE/MARKER LMP & 252810 & $1995 / 07 / 14$ & 32.25 & .00 & .00 & 32.25 \\
\hline CLEARANCE/MARKER LMP & 254394 & $1995 / 09 / 22$ & 32.25 & .00 & .00 & 32.25 \\
\hline 34300 CLEARANCE/MARKER LMP & 256753 & $1995 / 12 / 28$ & 21.50 & 1.95 & .00 & 23.45 \\
\hline REPAIR TYPE TOTAL & & & 290.25 & 14.64 & .00 & 304.89 \\
\hline 34330 MISC. LAMPS & 254394 & $1995 / 09 / 22$ & 21.50 & .00 & .00 & 21.50 \\
\hline REPAIR TYPE TOTAL & & & 21.50 & .00 & .00 & 22.50 \\
\hline 34400 STROBE LAMP & 248213 & $1995 / 01 / 12$ & 86.00 & .00 & .00 & 86.00 \\
\hline REPAIR TYE TOIM, & & & 86.00 & .00 & .00 & 86.00 \\
\hline
\end{tabular}


VEHICLE 3220 INT' EXPBASE TANDEM AXLE

\begin{tabular}{|c|c|c|c|c|c|c|}
\hline $\begin{array}{l}\text { REPAIR } \\
\text { CODE }\end{array}$ & $\begin{array}{l}\text { WORR } \\
\text { ORDER }\end{array}$ & $\begin{array}{c}\text { พO } \\
\text { COMPLETED }\end{array}$ & $\begin{array}{l}\text { LABOR } \\
\text { COST }\end{array}$ & $\begin{array}{l}\text { PARTS } \\
\text { COST }\end{array}$ & $\begin{array}{l}\text { OUTSIDE } \\
\text { COST }\end{array}$ & $\begin{array}{l}\text { TOTAL } \\
\text { COST }\end{array}$ \\
\hline PM/MINUS PM TOTAL & & & 311.75 & 21.08 & 68.38 & 401.21 \\
\hline 01410 HOSE CLAMPS/FITTINGS & 253215 & $1995 / 08 / 21$ & 64.50 & .55 & .00 & 65.05 \\
\hline REPAIR TYPE TOTAL & & & 64.50 & .55 & .00 & 65.05 \\
\hline $\begin{array}{l}01530 \text { HEATER SHUTOFF VALVE } \\
\text { REPAIR TYPE TOTAL }\end{array}$ & 251266 & $1995 / 06 / 12$ & $\begin{array}{l}75.25 \\
75.25\end{array}$ & $\begin{array}{l}2.20 \\
2.20\end{array}$ & $\begin{array}{l}.00 \\
.00\end{array}$ & $\begin{array}{l}77.45 \\
77.45\end{array}$ \\
\hline REPAIR SUB GROUP TOTAL & & & 139.75 & 2.75 & .00 & 142.50 \\
\hline $\begin{array}{l}\text { 0215R MIRROR, EXTERNAL } \\
\text { REPAIR TYPE TOTAL }\end{array}$ & 253035 & $1995 / 07 / 28$ & $\begin{array}{l}10.75 \\
10.75\end{array}$ & $\begin{array}{l}12.41 \\
12.41\end{array}$ & $\begin{array}{l}.00 \\
.00\end{array}$ & $\begin{array}{l}23.16 \\
23.16\end{array}$ \\
\hline REPAIR SUB GROUP TOTAL & & & 10.75 & 12.41 & .00 & 23.16 \\
\hline $\begin{array}{ll}0330 R & \text { AIR PRESSURE GAUGE } \\
03300 & \text { AIR PRESSURE GAUGE }\end{array}$ & $\begin{array}{l}255945 \\
255945\end{array}$ & $\begin{array}{l}1995 / 11 / 30 \\
1995 / 11 / 30\end{array}$ & $\begin{array}{r}.00 \\
86.00\end{array}$ & $\begin{array}{l}.00 \\
.00\end{array}$ & $\begin{array}{r}56.91 \\
.00\end{array}$ & $\begin{array}{l}56.91 \\
86.00\end{array}$ \\
\hline REPAIR TYPE TOTAL & & & 86.00 & .00 & 56.91 & 142.91 \\
\hline REPAIR SUB GROUP TOTAL & & & 86.00 & .00 & 56.91 & 242.91 \\
\hline $\begin{array}{l}07120 \text { DOOR LATCH ASSEMBLY } \\
\text { REPNIR TYPE TOTAL }\end{array}$ & 249988 & $1995 / 03 / 16$ & $\begin{array}{l}43.00 \\
43.00\end{array}$ & $\begin{array}{l}.00 \\
.00\end{array}$ & $\begin{array}{l}.00 \\
.00\end{array}$ & $\begin{array}{l}43.00 \\
43.00\end{array}$ \\
\hline $\begin{array}{l}\text { 0797R DMPBODY SPILL SHIELD } \\
\text { REPAIR TXPE TOTAL }\end{array}$ & 251778 & $1995 / 06 / 12$ & $\begin{array}{l}193.50 \\
193.50\end{array}$ & $\begin{array}{l}18.35 \\
18.35\end{array}$ & $\begin{array}{l}23.84 \\
23.84\end{array}$ & $\begin{array}{l}235.69 \\
235.69\end{array}$ \\
\hline $\begin{array}{l}\text { 0799R PAINTING } \\
\text { REPAIR TYPE TOTAL }\end{array}$ & 251778 & $1995 / 06 / 12$ & $\begin{array}{l}43.00 \\
43.00\end{array}$ & $\begin{array}{l}.00 \\
.00\end{array}$ & $\begin{array}{l}.00 \\
.00\end{array}$ & $\begin{array}{l}43.00 \\
43.00\end{array}$ \\
\hline REPAIR SUB GROUP TOTAL & & & 279.50 & 18.35 & 23.84 & 321.69 \\
\hline REPAIR GROUP TOTAL & & & 516.00 & 33.51 & 80.75 & 630.26 \\
\hline 13500 AIR COMPRESSOR & 250952 & $1995 / 04 / 26$ & 86.00 & 13.98 & .00 & 99.98 \\
\hline REPAIR TYPE TOTAL & & & 86.00 & 13.98 & .00 & 99.98 \\
\hline 13550 AIR DRYER ASSEMBLY & 250685 & $1995 / 06 / 12$ & 43.00 & .00 & 25.07 & 68.07 \\
\hline 13551 AIR DRYER ASSEMBLY & 250685 & $1995 / 06 / 12$ & 21.50 & .00 & .00 & 21.50 \\
\hline REPAIR TYPE TOTAL & & & 64.50 & .00 & 25.07 & 89.57 \\
\hline $\begin{array}{l}13800 \text { SLACK ADJUSTERS } \\
\text { REPAIR TYPE TOTAL }\end{array}$ & 255091 & $1995 / 10 / 25$ & $\begin{array}{l}75.25 \\
75.25\end{array}$ & $\begin{array}{l}.00 \\
.00\end{array}$ & $\begin{array}{l}.00 \\
.00\end{array}$ & $\begin{array}{l}75.25 \\
75.25\end{array}$ \\
\hline 1385R SPRING PARKING BRAKE & 251910 & $1995 / 06 / 02$ & 150.50 & 141.32 & 136.17 & 427.99 \\
\hline REPAIR TYPE TOTAL & & & 150.50 & 141.32 & 136.17 & 427.99 \\
\hline REPAIR SUB GROUP TOTAL & & & 376.25 & 155.30 & 161.24 & 692.79 \\
\hline
\end{tabular}


VEHICLE 3220 INT' EXPBASE TANDEM AXLE

\begin{tabular}{|c|c|c|c|c|c|c|}
\hline $\begin{array}{l}\text { REPAIR } \\
\text { CODE } \quad \text { DESCRIPTION }\end{array}$ & $\begin{array}{r}\text { WORK } \\
\text { ORDER }\end{array}$ & $\begin{array}{c}\text { WO } \\
\text { COMPLETED }\end{array}$ & $\begin{array}{r}\text { LABOR } \\
\text { COST }\end{array}$ & $\begin{array}{r}\text { PARTS } \\
\text { COST }\end{array}$ & $\begin{array}{l}\text { OUTSIDE } \\
\text { COST }\end{array}$ & $\begin{array}{l}\text { TOTAL } \\
\text { COST }\end{array}$ \\
\hline REPAIR SUB GROUP TOTAI. & & & 129.00 & 3.75 & 3.86 & 136.61 \\
\hline 7721R HYD TANK FILTER & 244649 & $1994 / 08 / 24$ & 53.75 & .00 & .00 & 53.75 \\
\hline REPAIR TYPE TOTAL & & & 53,75 & .00 & .00 & 53.75 \\
\hline REPAIR SUB GROUP TOTAL & & & 53.75 & .00 & .00 & 53.75 \\
\hline REPAIR GROUP TOTAL & & & 182.75 & 3.75 & 3.86 & 190.36 \\
\hline 98000 MISC INSIDE WORK & M05306 & $1.994 / 03 / 01$ & .00 & .00 & 2.56 & 2.56 \\
\hline REPAIR TYPE TOTAL & & & .00 & .00 & 2.56 & 2.56 \\
\hline 98200 GENERAL GREASING & M05155 & $1994 / 01 / 21$ & 86.00 & .80 & .00 & 86.80 \\
\hline 98200 GENERAL GREASING & M05440 & $1994 / 03 / 15$ & 107.50 & .80 & .00 & 108.30 \\
\hline 98200 GENERAL GREASING & M05605 & $1994 / 04 / 29$ & 118.25 & 3.44 & .00 & 121.69 \\
\hline 98200 GENERAL GREASING & M05660 & $1994 / 05 / 10$ & 107.50 & .80 & 1.28 & 109.58 \\
\hline 98200 GENERAL GREASING & $M 05706$ & $1994 / 06 / 01$ & 64.50 & 2.81 & .00 & 67.31 \\
\hline 98200 GENERAL GREASING & M05980 & $1994 / 08 / 16$ & 64.50 & .00 & 20.40 & 84.90 \\
\hline 98200 GENERAL GREASING & M05983 & $1994 / 08 / 17$ & 64.50 & .80 & .00 & 65.30 \\
\hline 98200 GENERAL GREASINO & M06317 & $1994 / 11 / 09$ & 139.75 & 11.84 & .00 & 151.59 \\
\hline 98200 GENERAL GREASING & M06447 & $1994 / 12 / 14$ & 86.00 & .80 & 4.92 & 91.72 \\
\hline REPAIR TYPE TOTAL & & & 838.50 & 22.09 & 26.60 & 887.19 \\
\hline 9860I TRUCK INSPECTION & 244649 & $1994 / 08 / 24$ & 172.00 & .00 & .00 & 172.00 \\
\hline REPAIR TYPE TOTAL & & & 172.00 & .00 & .00 & 172.00 \\
\hline REPAIR SUB GROUP TOTAL & & & $1,010.50$ & 22.09 & 29.16 & $1,061.75$ \\
\hline 99500 ACCESSORIES GROUP & 244761 & $1994 / 09 / 14$ & .00 & .00 & .00 & .00 \\
\hline REPAIR TYPE TOTAL & & & .00 & .00 & .00 & .00 \\
\hline REPAIR SUB GROUP TOTAL & & & .00 & .00 & .00 & .00 \\
\hline REPAIR GROUP TOTAL & & & $1,010.50$ & 22.09 & 29.16 & $1,061.75$ \\
\hline PM/MINUS PM TOTAL & & & $4,181.75$ & 186.99 & $1,148.68$ & $5,517,42$ \\
\hline ENDYR TOTAL & & & $4,504.25$ & 227.11 & $1,165.32$ & $5,896.68$ \\
\hline 1115L PMA & 250328 & $1995 / 03 / 29$ & 107.50 & 10.10 & 42.64 & 160.24 \\
\hline $1115 L \quad$ PMA & 255091 & $1995 / 10 / 25$ & 172.00 & 10.98 & 25.74 & 208.72 \\
\hline REPAIR TYPE TOTAL & & & 279.50 & 21.08 & 68.38 & 368.96 \\
\hline 11201, PMB & 253215 & $1995 / 08 / 21$ & 32.25 & .00 & .00 & 32.25 \\
\hline REPAIR TYPE TOTAL & & & 32.25 & .00 & .00 & 32.25 \\
\hline REPAIR SUB GROUP TOTAL & & & 311.75 & 21.08 & 68.38 & 401.21 \\
\hline REPAIR GROUP TOTAL & & & 311.75 & 21.08 & 68.38 & 401.21 \\
\hline
\end{tabular}


VEHICLE 3220 INT' EXPBASE TINDEM AXLE

\begin{tabular}{|c|c|c|c|c|c|c|}
\hline $\begin{array}{l}\text { REPAIR } \\
\text { CODE }\end{array}$ & $\begin{array}{r}\text { WORK } \\
\text { ORDER }\end{array}$ & $\begin{array}{c}\text { WO } \\
\text { COMPLETED }\end{array}$ & $\begin{array}{r}\text { LABOR } \\
\text { COST }\end{array}$ & $\begin{array}{r}\text { PARTS } \\
\operatorname{COST} T\end{array}$ & $\begin{array}{l}\text { OUTS IDE } \\
\text { CosT }\end{array}$ & $\begin{array}{l}\text { TOTAL } \\
\text { COST }\end{array}$ \\
\hline 4212R COOLANT FILTER & 244649 & $1994 / 08 / 24$ & 118.25 & 48.05 & 40.70 & 207.00 \\
\hline REPAIR TYPE TOTAL & & & 118.25 & 48.05 & 40.70 & 207.00 \\
\hline REPAIR SUB GROUP TOTAL & & & 161.25 & 48.05 & 40.70 & 250.00 \\
\hline 4410R FILTER， PRIMARY & 244649 & $1994 / 08 / 24$ & 10.75 & .00 & 4.69 & 15.44 \\
\hline REPAIR TYPE TOTAL & & & 10.75 & .00 & 4.69 & 15.44 \\
\hline 4411R FILTER, SECONDARY & 244649 & $1994 / 08 / 24$ & 10.75 & .00 & 7.43 & 18.18 \\
\hline REPAIR TYPE TOTAL & & & 10.75 & .00 & 7.43 & 18.18 \\
\hline REPAIR SUB GROUP TOTAL & & & 21.50 & .00 & 12.12 & 33.62 \\
\hline 4562 I OIL FILTER ASSEMBLY & 247663 & $1994 / 12 / 19$ & 43.00 & .00 & .00 & 43.00 \\
\hline REPAIR TYPE TOTAL & & & 43.00 & .00 & .00 & 43.00 \\
\hline REPAIR SUB GROUP TOTAL & & & 43.00 & .00 & .00 & 43.00 \\
\hline REPAIR GROUP TOTAL & & & 376.25 & 48.05 & 63.70 & 488.00 \\
\hline 51000 ACCESSORIES, GENERAL & 240993 & $1994 / 04 / 12$ & 526.75 & .00 & .00 & 526.75 \\
\hline 51000 ACCESSORIES, GENERAL & 243064 & $1994 / 06 / 21$ & $\$ 3.00$ & 3.27 & .00 & 46.27 \\
\hline REPAIR TYPE TOTAL & & & 569.75 & 3.27 & .00 & 573.02 \\
\hline $5110 R$ TARPALIN ASSEMBLY & 240993 & $1994 / 04 / 12$ & .00 & .00 & 852.72 & 852.72 \\
\hline REPAIR TYPE TOTAL & & & .00 & .00 & 852.72 & 852.72 \\
\hline REPAIR SUB GROUP TOTAL & & & 569.75 & 3.27 & 852.72 & 1.425 .74 \\
\hline 52210 RADIO, AM/FM & 244712 & $1994 / 09 / 22$ & 64.50 & .00 & 104.00 & 168.50 \\
\hline 52210 RADIO, AM/FM & 245791 & $1994 / 10 / 17$ & 182.75 & .00 & .00 & 182.75 \\
\hline REPAIR TYPE TOTAL & & & 247.25 & .00 & 104.00 & 351.25 \\
\hline 52500 MISC SWITHCHES & 246233 & $1994 / 10 / 19$ & .00 & .00 & 24.24 & 24.24 \\
\hline REPAIR TYPE TOTAL & & & .00 & .00 & 24.24 & 24.24 \\
\hline REPAIR SUB GROUP TOTAL & & & 247.25 & .00 & 128.24 & 375.49 \\
\hline 54100 SNOW PLOW FRAME & 239230 & $1994 / 02 / 04$ & 64.50 & .00 & .00 & 64.50 \\
\hline REPAIR TYPE TOTAL & & & 64.50 & .00 & .00 & 64.50 \\
\hline REPAIR SUB GROUP TOTAL & & & 64.50 & .00 & .00 & 64.50 \\
\hline RFFAIR GROUP TOTAI, & & & 881.50 & 3.27 & 980.96 & $1,865.73$ \\
\hline 7476R CABLE, CONTL LEVER & 243876 & $1994 / 07 / 29$ & 64.50 & .00 & .00 & 64.50 \\
\hline 74760 CABLE, CONTL LEVER & 243876 & $1994 / 07 / 29$ & .00 & 1.90 & 3.86 & 5.76 \\
\hline 74760 CABLE, CONTL LEVER & 243998 & $1994 / 07 / 29$ & 64.50 & 1.85 & .00 & 66.35 \\
\hline REPAIR TYYPE TOTAL & & & 129.00 & 3.75 & 3.86 & 136.61 \\
\hline
\end{tabular}


VEHICLE 3220 INT EXPBASE TANDEM AXLE

\begin{tabular}{|c|c|c|c|c|c|c|}
\hline $\begin{array}{c}\text { REPAIR } \\
\text { CODE }\end{array}$ & $\begin{array}{r}\text { WORK } \\
\text { ORDER }\end{array}$ & $\begin{array}{c}\text { WO } \\
\text { COMPLETED }\end{array}$ & $\begin{array}{r}\text { LABOR } \\
\text { COST }\end{array}$ & $\begin{array}{r}\text { PARTS } \\
\text { COST }\end{array}$ & $\begin{array}{l}\text { OUTSIDE } \\
\text { COST }\end{array}$ & $\begin{array}{r}\text { TOTAL } \\
\text { COST }\end{array}$ \\
\hline READTAMP & 239025 & $1334 / 02 / 01$ & 118.25 & .00 & .00 & 118.25 \\
\hline REPAIR TYPE TOTAL & & & 118.25 & .00 & .00 & 118.25 \\
\hline 34100 STOPLAMP & 245198 & $1994 / 09 / 14$ & 21.50 & .00 & .00 & 21.50 \\
\hline REPAIR TYPE TOTAL & & & 21.50 & .00 & .00 & 21.50 \\
\hline 3425R BACKUP LAMP & 246763 & $1994 / 11 / 10$ & .00 & 9.16 & .00 & 9.16 \\
\hline REPAIR TYPE TOTAL & & & .00 & 9.16 & .00 & 9.16 \\
\hline CLEARANCE/MARKER LMP & 238204 & $1994 / 01 / 10$ & 53.75 & .00 & 22.75 & 76.50 \\
\hline 34300 CLEARANCE/MARKER LMP & 240079 & $1994 / 03 / 03$ & 21.50 & .00 & .00 & 21.50 \\
\hline 34300 CLEARANCE/MARKER LMP & 240921 & $1994 / 04 / 04$ & 64.50 & .00 & .00 & 64.50 \\
\hline 34300 CLEARANCE/MARKER LMP & 243876 & $1994 / 07 / 29$ & 193.50 & .00 & .00 & 193.50 \\
\hline 34300 CLEARANCE/MARKER IMP & 245791 & $1994 / 10 / 17$ & 96.75 & .00 & .00 & 96.75 \\
\hline 3430R CLEARANCE/MARKER LMP & 247734 & $1994 / 12 / 19$ & 21.50 & 2.05 & 11.76 & 35.31 \\
\hline REPAIR TYPE TOTAL & & & 451.50 & 2.05 & 34.52 & 488.06 \\
\hline 34340 FLOOD LAMPS & 243876 & $1994 / 07 / 29$ & .00 & .00 & 9.83 & 9.83 \\
\hline REPAIR TYPE TOTAL & & & .00 & .00 & 9.83 & 9.83 \\
\hline 34400 STROBE LAMP & 239065 & $1994 / 02 / 01$ & 32.25 & .00 & .00 & 32.25 \\
\hline 34400 STROBE LLAMP & 239479 & $1994 / 02 / 14$ & 172.00 & .00 & .00 & 172.00 \\
\hline STROBE LAMP & 239384 & $1994 / 02 / 14$ & 21.50 & .00 & .00 & 21.50 \\
\hline 34400 STROBE LAMP & 247806 & $1994 / 12 / 20$ & 86.00 & .00 & .00 & 86.00 \\
\hline REPAIR TYPE TOTAL & & & 311.75 & .00 & .00 & 311.75 \\
\hline 3450R SANDER/WORK LAMP & 246763 & $1994 / 11 / 10$ & 75.25 & .00 & .00 & 75.25 \\
\hline 3450R SANDER/WORK LAMP & 246876 & $1994 / 11 / 16$ & 43.00 & 9.23 & .00 & 52.23 \\
\hline REPAIR TYPE TOTAL & & & 118.25 & 9.23 & .00 & 127.48 \\
\hline 3455R LAMP SWITCH(S) & 243876 & $1994 / 07 / 29$ & 21.50 & .00 & .00 & 21.50 \\
\hline REPAIR TYPE TOTAL & & & 21.50 & .00 & .00 & 21.50 \\
\hline REPAIR SUB GROUP TOTAL & & & $1,042.75$ & 20.44 & 44.34 & $1,107.53$ \\
\hline REPAIR GROUP TOTAL & & & $1,268.50$ & 94.13 & 44.34 & $1,406.97$ \\
\hline 41000 INTAKE SYSTEM & 240427 & $1994 / 03 / 11$ & 139.75 & .00 & .00 & 139.75 \\
\hline REPAIR TXPE TOTAL & & & 139.75 & .00 & .00 & 139.75 \\
\hline INTAKE DUCTING, AC & 240079 & $1994 / 03 / 03$ & 10.75 & .00 & .00 & 10.75 \\
\hline$\$ 1460$ INTAKE DUCTING, AC & 240427 & $1994 / 03 / 11$ & .00 & .00 & 10.88 & 10.88 \\
\hline REPAIR TYPE TOTAL & & & 10.75 & .00 & 10.88 & 21.63 \\
\hline REPAIR SUB GROUP TOTAL & & & 150.50 & .00 & 10.88 & 161.38 \\
\hline 42010 RADIATOR & 240079 & $1994 / 03 / 03$ & 43.00 & .00 & .00 & 43.00 \\
\hline REPAIR TYPE TOTAL & & & 43.00 & .00 & .00 & 43.00 \\
\hline
\end{tabular}

43.00

.00

43.00 


\begin{tabular}{|c|c|c|c|c|c|c|}
\hline $\begin{array}{l}\text { REPAIR } \\
\text { CODE }\end{array}$ & $\begin{array}{l}\text { WORK } \\
\text { ORDER }\end{array}$ & $\begin{array}{c}\text { WO } \\
\text { COMPLETED }\end{array}$ & $\begin{array}{r}\text { LABOR } \\
\text { COST }\end{array}$ & $\begin{array}{r}\text { PARTS } \\
\text { COST }\end{array}$ & $\begin{array}{l}\text { OUTS IDE } \\
\text { COST }\end{array}$ & $\begin{array}{l}\text { TOTAL } \\
\text { COST }\end{array}$ \\
\hline 17010 TIRE, FLAT & 245567 & $1994 / 09 / 21$ & 43.00 & .00 & .00 & 43.00 \\
\hline REPAIR TYPE TOTAL & & & 43.00 & .00 & .00 & 43.00 \\
\hline REPAIR SUB GROUP TOTAL & & & 43.00 & .00 & .00 & 43.00 \\
\hline REPAIR SUB GROUP TOTAL & & & 129.00 & .00 & 26.66 & 155.66 \\
\hline REPAIR GROUP TOTAL & & & 172.00 & .00 & 26.66 & 198.66 \\
\hline $\begin{array}{l}22070 \text { GASKET, AXLE SHAFT } \\
\text { REPAIR TYPE TOTAL }\end{array}$ & 246020 & $1994 / 10 / 10$ & $\begin{array}{l}43.00 \\
43.00\end{array}$ & $\begin{array}{l}.00 \\
.00\end{array}$ & $\begin{array}{l}.00 \\
.00\end{array}$ & $\begin{array}{l}43.00 \\
43.00\end{array}$ \\
\hline REPAIR SUB GROUP TOTAL & & & 43.00 & .00 & .00 & 43.00 \\
\hline $\begin{array}{l}2300 \mathrm{C} \text { CLUTCH ASSEMBLY } \\
\text { REPAIR TYPE TOTAL }\end{array}$ & 244649 & $1994 / 08 / 24$ & $\begin{array}{l}43.00 \\
43.00\end{array}$ & $\begin{array}{l}.00 \\
.00\end{array}$ & $\begin{array}{l}.00 \\
.00\end{array}$ & $\begin{array}{l}\$ 3.00 \\
43.00\end{array}$ \\
\hline 2301L PRESSURE PLATE & 242282 & $1994 / 05 / 23$ & 43.00 & .00 & .00 & 43.00 \\
\hline 23011. PRESSURE PLATE & 247663 & $1994 / 12 / 19$ & 43.00 & .00 & .00 & 43.00 \\
\hline REPAIR TYPE TOTAL & & & 86.00 & .00 & .00 & 86.00 \\
\hline REPAIR SUB GROUP TOTAL & & & 129.00 & .00 & .00 & 129.00 \\
\hline $\begin{array}{l}\text { 2787L LINKAGE } \\
\text { REPAIR TYPE TOTAL }\end{array}$ & 244096 & $1994 / 08 / 01$ & $\begin{array}{l}86.00 \\
86.00\end{array}$ & $\begin{array}{l}.00 \\
.00\end{array}$ & $\begin{array}{l}.00 \\
.00\end{array}$ & $\begin{array}{l}86.00 \\
86.00\end{array}$ \\
\hline REPAIR SUB GROUP TOTAL & & & 86.00 & .00 & .00 & 86.00 \\
\hline REPAIR GROUP TOTAL & & & 258.00 & .00 & .00 & 258.00 \\
\hline $\begin{array}{r}32010 \text { BATTERY } \\
\text { REPAIR TYPE TOTAL }\end{array}$ & 238878 & $1994 / 01 / 25$ & 21.50 & 66.42 & .00 & 87.92 \\
\hline 32020 BATTERY CABLE & 238878 & $1994 / 01 / 25$ & 64.50 & 6.54 & .00 & 71.04 \\
\hline REPAIR TYPE TOTAL & & & 64.50 & 6.54 & .00 & 71.04 \\
\hline 32060 BATTERY HOLDOWNS & 238878 & $1994 / 01 / 25$ & 53.75 & .73 & .00 & 54.48 \\
\hline REPAIR TYPE TOTAL & & & 53.75 & .73 & .00 & 54.48 \\
\hline REPAIR SUB GROUP TOTAL & & & 139.75 & 73.69 & .00 & 213.44 \\
\hline 33350 IGNITION SWITCH & 246233 & $1994 / 10 / 19$ & 86.00 & .00 & .00 & 86.00 \\
\hline REPAIR TYPE TOTAL & & & 86.00 & .00 & .00 & 86.00 \\
\hline REPAIR SUB GROUP TOTAL & & & 86.00 & .00 & .00 & 86.00 \\
\hline
\end{tabular}




\begin{tabular}{|c|c|c|c|c|c|c|}
\hline $\begin{array}{l}\text { REPAIR } \\
\text { CODE }\end{array}$ & $\begin{array}{l}\text { WORK } \\
\text { ORDER }\end{array}$ & $\begin{array}{c}\text { W० } \\
\text { COMPLETED }\end{array}$ & $\begin{array}{r}\text { LABOR } \\
\text { COST }\end{array}$ & $\begin{array}{r}\text { PARTS } \\
\cos \mathrm{T}\end{array}$ & $\begin{array}{l}\text { OUTSIDE } \\
\text { Cost }\end{array}$ & $\begin{array}{r}\text { TOTAL } \\
\text { COST }\end{array}$ \\
\hline 66000 SP EQUIP ACCESSORIES & 237740 & $1993 / 12 / 21$ & 75.25 & .00 & .00 & 75.25 \\
\hline $6600 R \quad S P$ EQUIP ACCESSORIES & 237740 & $1993 / 12 / 21$ & .00 & 3.56 & .00 & 3.56 \\
\hline REPAIR TYPE TOTAL & & & 75.25 & 3.56 & .00 & 78.81 \\
\hline REPAIR SUB GROUP TOTAL & & & 75.25 & 3.56 & .00 & 78.81 \\
\hline REPAIR GROUP TOTAL & & & 75.25 & 3.56 & .00 & 78.81 \\
\hline HYDRAULIC VALVES & 236759 & $1993 / 11 / 19$ & 107.50 & .00 & .00 & 107.50 \\
\hline REPAIR TYPE TOTAL & & & 107.50 & .00 & .00 & 107.50 \\
\hline REPAIR SUB GROUP TOTAL & & & 107.50 & .00 & .00 & 107.50 \\
\hline REPAIR GROUP TOTAL & & & 107.50 & .00 & .00 & 107.50 \\
\hline 9830I NEW VEHICLE PREP. & 231147 & $1993 / 09 / 17$ & .00 & .00 & 271.20 & 271.20 \\
\hline 98300 NEW VEHICLE PREP. & 231147 & $1993 / 09 / 17$ & $2,655.25$ & 266.59 & 207.69 & $3,129,53$ \\
\hline 98300 NEW VEHICLE PREP. & 235739 & $1993 / 10 / 01$ & $\begin{array}{r}64.50 \\
2.719 .75\end{array}$ & $\begin{array}{r}.00 \\
266.59\end{array}$ & $\begin{array}{l}413.99 \\
892.88\end{array}$ & $\begin{array}{r}478.49 \\
3.879 .22\end{array}$ \\
\hline 98500 SEASONAI, PREP & 231147 & $1993 / 09 / 17$ & 75.25 & .00 & 301.92 & 377.17 \\
\hline REPAIR TYPE TOTAI. & & & 75.25 & .00 & 301.92 & 377.17 \\
\hline REPAIR SUB GROUP TOTAL & & & $2,795.00$ & 266.59 & $1,194.80$ & 4.256 .39 \\
\hline REPAIR GROUP TOTAL & & & $2,795.00$ & 266.59 & $1,194,80$ & $4,256.39$ \\
\hline PM/MINUS PM TOTAL & & & $3,278.75$ & 272.50 & $1,693.99$ & $5,245.24$ \\
\hline ENDYR TOTAL & & & $3,421.75$ & 284.01 & $1,719.99$ & $5,425.75$ \\
\hline $1115 \mathrm{~L}$ & 240079 & $1994 / 03 / 03$ & 193.50 & 28.32 & .00 & 221.82 \\
\hline $1115 \mathrm{~L}$ PMA & 245567 & $1994 / 09 / 21$ & 86.00 & 10.90 & 16.64 & 113.54 \\
\hline REPAIR TYPE TOTAL & & & 279.50 & 39.22 & 16.64 & 335.36 \\
\hline $11201, \quad$ PMB & 244649 & $1994 / 08 / 24$ & 43.00 & .90 & .00 & $\begin{array}{l}43.90 \\
43.90\end{array}$ \\
\hline REPAIR TYPE TOTAL & & & 43.00 & .90 & .00 & 43.90 \\
\hline REPAIR SUB GROUP TOTAL & & & 322.50 & 40.12 & 16.64 & 379.26 \\
\hline REPAIR GROUP TOTAL & & & 322.50 & 40.12 & 16.64 & 379.26 \\
\hline PM/MINUS PM TOTAL & & & 322.50 & 40.12 & 16.64 & 379.26 \\
\hline 0787R MISC DECAL(S) & 245137 & $1994 / 09 / 14$ & 32.25 & 15.70 & .00 & 47.95 \\
\hline REPAIR TYPE TOTAL & & & 32.25 & 15.70 & .00 & 47.95 \\
\hline RFPMIR SUB GROUP TOTAL & & & 32.25 & 15.70 & .00 & 17.95 \\
\hline REPAIR GROUP TOTAL & & & 32.25 & 15.70 & .00 & 47.95 \\
\hline
\end{tabular}


VEHICLE 3220 INT' EXPBASE TANDEM NXLE

\begin{tabular}{|c|c|c|c|c|c|c|}
\hline $\begin{array}{l}\text { REPAIR } \\
\text { CODE }\end{array}$ & $\begin{array}{r}\text { WORK } \\
\text { ORDER }\end{array}$ & $\begin{array}{c}\text { WO } \\
\text { COMPLETED }\end{array}$ & $\begin{array}{r}\text { LABOR } \\
\text { COST }\end{array}$ & $\begin{array}{l}\text { PARTS } \\
\text { COST }\end{array}$ & $\begin{array}{l}\text { OUTSIDE } \\
\text { COST }\end{array}$ & $\begin{array}{l}\text { TOTAL } \\
\text { COST }\end{array}$ \\
\hline $\begin{array}{l}1115 L \text { PMA } \\
\text { REPAIR TYPE TOTAL }\end{array}$ & 238003 & $1993 / 12 / 22$ & $\begin{array}{l}143.00 \\
143.00\end{array}$ & $\begin{array}{l}11.51 \\
11.51\end{array}$ & $\begin{array}{l}26.00 \\
26.00\end{array}$ & $\begin{array}{l}180.51 \\
180.51\end{array}$ \\
\hline REPAIR SUB GROUP TOTAL & & & 143.00 & 11.51 & 26.00 & 180.51 \\
\hline REPAIR GROUP TOTAL & & & 143.00 & 11.51 & 26.00 & 180.51 \\
\hline PM/MINUS PM TOTAL & & & 143.00 & 21.51 & 26.00 & 180.51 \\
\hline 13600 AIR SUPPLY TANKS & 236872 & $1993 / 11 / 17$ & 96.75 & .00 & 30.24 & 126.99 \\
\hline REPAIR TYPE TOTAL & & & 96.75 & .00 & 30.24 & 126.99 \\
\hline 1365R AIR VALVES & 231147 & $1993 / 09 / 17$ & 64.50 & .00 & 14.53 & 79.03 \\
\hline REPAIR TXPE TOTAL & & & 64.50 & .00 & 14.53 & 79.03 \\
\hline REPAIR SUB GROUP TOTAL & & & 161.25 & .00 & 44.77 & 206.02 \\
\hline 180OR WHEEL/RIM & 236759 & $1993 / 11 / 19$ & .00 & .00 & 347.21 & 347.21 \\
\hline REPAIR TYPE TOTAL & & & .00 & .00 & 347.21 & 347.21 \\
\hline REPAIR SUB GROUP TOTAL & & & .00 & .00 & 347.21 & 347.21 \\
\hline REPAIR GROUP TOTAL & & & 161.25 & .00 & 391.96 & 553.23 \\
\hline 4320R EXHAUST TUBING & 231147 & $1993 / 09 / 17$ & .00 & .00 & 62.09 & 62.09 \\
\hline REPAIR TYPE TOTAL & & & .00 & .00 & 62.09 & 62.09 \\
\hline REPAIR SUB GROUP TOTAL & & & .00 & .00 & 62.09 & 62.09 \\
\hline REPAIR GROUP TOTAL & & & .00 & .00 & 62.09 & 62.09 \\
\hline 51050 AIR HORN ASSEMBLY & 238026 & $1993 / 12 / 22$ & 75.25 & .00 & .00 & 75.25 \\
\hline REPAIR 'I'YPE TOTAL & & & 75.25 & .00 & .00 & 75.25 \\
\hline REPAIR SUB GROUP TOTAL & & & 75.25 & .00 & .00 & 75.25 \\
\hline 52000 ACCESSORY, ELECTRIC & 236705 & $1993 / 11 / 12$ & 21.50 & .00 & .00 & 21.50 \\
\hline REPAIR TYPE TOTAL & & & 21.50 & .00 & .00 & 21.50 \\
\hline $52250 \quad 2$ - WAY RADIO & 236759 & $1993 / 11 / 19$ & 43.00 & 2.35 & .00 & 45.35 \\
\hline REPAIR TYPE TOTAL & & & 43.00 & 2.35 & .00 & 45.35 \\
\hline REPAIR SUB GROUP TOTAL & & & 64.50 & 2.35 & .00 & 66.85 \\
\hline 5910R PINTLE HOOK & 231147 & $1993 / 09 / 17$ & .00 & .00 & 45.12 & 45.12 \\
\hline REFAIR TYPE TOTAL & & & .00 & .00 & 45.12 & 45.12 \\
\hline REPMIR SUB GROUP TOTAL & & & .00 & .00 & 45.12 & 45.12 \\
\hline REPAIR GROUP TOTAL & & & 139.75 & 2.35 & 45.12 & 187.22 \\
\hline
\end{tabular}


Appendix 6

Oil Analysis Results 
Summary of All Oil Analyses

\begin{tabular}{|l|c|c|c|}
\hline Unit \# & Miles at sample & Pass/Fail & Was oil changed? \\
\hline 3220 & 37065 & Pass & \\
\hline & 40485 & Fail & Yes \\
\hline & 48880 & Pass & \\
\hline & 57112 & Pass & Yes \\
\hline & 65403 & Pass & \\
\hline & 69405 & Pass & \\
\hline & 72051 & Pass & \\
\hline & 81301 & Pass & \\
\hline & 87817 & Fail & Yes \\
\hline 3221 & & & \\
\hline & 28992 & Fail & Yes \\
\hline & 35381 & Pass & \\
\hline & 41029 & Pass & \\
\hline & 45090 & Fail & Yes \\
\hline & 50681 & Pass & \\
\hline & 58347 & Pass & \\
\hline 3228 & 60715 & Fail & \\
\hline & 21564 & Yes \\
\hline & 26740 & Pass & \\
\hline & 29638 & Pass & \\
\hline & 32803 & Pass & Yes \\
\hline
\end{tabular}


VARGIAR-CMT $=-1$ $(612) 888-4121 \cdot(800) 352-2812$

SCHEDULED OIL SAMPLING WEAR ANALYSIS PEPORT

Sample For

HENNEPIN CTY HIGHWAY DEPT

JIM ALLEN

320 WASHINGTON AVE. S.

HOPKINS, MN, 55343

$\begin{array}{ll}\text { Serial \#: } & 4 \text { A32254 } \\ \text { Compartment: } & \text { Diesel Engine } \\ \text { Unil \#: } & 3220 \\ \text { Model \#: } & \end{array}$

Sample \#: $\quad 96120446$

Date Received: $12 / 03 / 96$

Oil Brand: Caterpillar

Oil Blend: Diesel Engine 0 Oil Weight: $\quad 15 W 40$

DETROIT 6V92

\begin{tabular}{|c|c|c|c|c|c|c|c|c|c|c|c|c|c|c|c|c|c|c|c|c|c|c|}
\hline \multicolumn{6}{|c|}{ ICURRENT SAMPLE INFORMATION } & \multicolumn{9}{|c|}{ If } & \multirow{2}{*}{\multicolumn{4}{|c|}{$\begin{array}{l}\text { INFRAAEO ANALYSIS } \\
\text { PERCENT OF ALLOWABLE }\end{array}$}} & \multirow{2}{*}{\multicolumn{4}{|c|}{$\begin{array}{l}\text { PHYSICAL } \\
\text { TESTS }\end{array}$}} \\
\hline \multirow{2}{*}{$\begin{array}{l}\text { DATE } \\
\text { SAMPLE } \\
\text { TAKEN }\end{array}$} & \multicolumn{2}{|c|}{ CHANGED } & \multirow{2}{*}{$\begin{array}{l}\text { QUAATS } \\
\text { OIL } \\
\text { ADDED }\end{array}$} & \multirow{2}{*}{$\begin{array}{l}\text { HOUAS } \\
\text { ON } \\
\text { METEA }\end{array}$} & \multirow{2}{*}{$\begin{array}{l}\text { HOURS } \\
\text { ON } \\
\text { OIL }\end{array}$} & \multirow{2}{*}{$\begin{array}{c}\text { CU } \\
\text { Coppor } \\
\text { PPM }\end{array}$} & \multirow{2}{*}{$\begin{array}{c}\text { FE } \\
\text { lion } \\
\text { PPM }\end{array}$} & \multirow{2}{*}{$\begin{array}{c}\text { CP } \\
\text { Chromlum } \\
\text { PPM }\end{array}$} & \multirow{2}{*}{$\begin{array}{c}\text { AL } \\
\text { Aluminum } \\
\text { PPM }\end{array}$} & \multirow{2}{*}{$\begin{array}{c}\text { SI } \\
\text { sitloon } \\
\text { PPM }\end{array}$} & \multirow{2}{*}{$\begin{array}{l}\text { PB } \\
\text { Load } \\
\text { PPM }\end{array}$} & \multirow{2}{*}{$\begin{array}{l}\text { NA } \\
\text { Sodhum } \\
\text { PPM }\end{array}$} & \multirow{2}{*}{$\begin{array}{c}\text { MO } \\
\text { Mointadenum } \\
\text { PPM }\end{array}$} & \multirow{2}{*}{$\begin{array}{c}\text { SN } \\
\text { Iin } \\
\text { PPM }\end{array}$} & & & & & & & & \\
\hline & Oil & Filler & & & & & & & & & & & & & soot & $\begin{array}{l}\text { unation } \\
\%\end{array}$ & idation & intration & & & & \\
\hline$\longdiv { 2 / 0 2 / 9 6 }$ & $\bar{Y}$ & $\mathrm{Y}$ & & 87817 & & 15 & 128 & 4 & 2 & 8 & 28 & 9 & 2 & 23 & 180 & 65 & 93 & 65 & & & $\bar{N}$ & $\bar{N}$ \\
\hline
\end{tabular}

Interpretation

The analysis of the oil sample taken from your Diesel Engine indicates that Iron and Lead are

slightly high; Soot is high. When the MILES/HOURS ON DIL data are unknown, we use the full oil

change interval to analyze the sample. If your HouRs oN OIL reading is known please call us, we

will reinterpret your results.

Recommendation

Continue to monitor compartment by sampling at recommended intervals. Be sure to fill out labels as complete as possible!

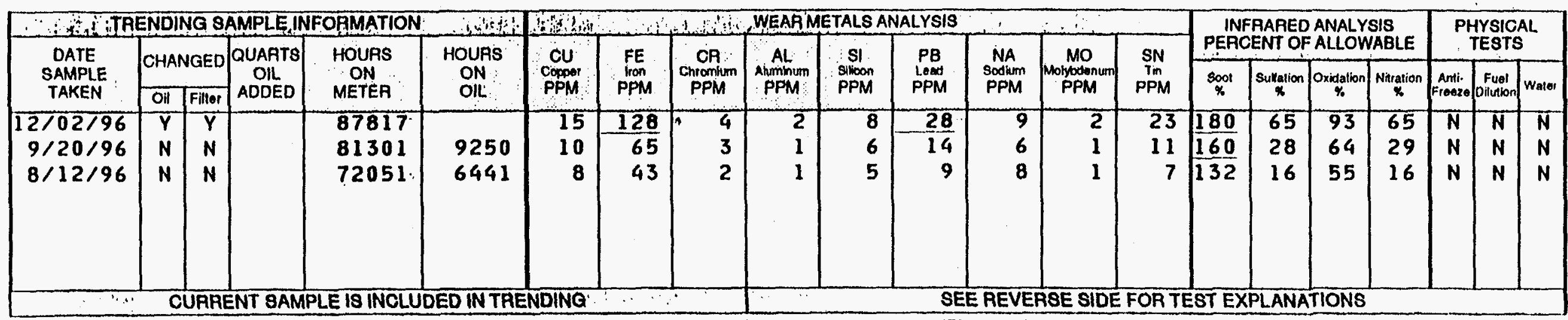


HEPANETIN COUNTY

JII ALLEN

320 'AESHINGTON AVENUE

HDEKIMS HH SSZ43
ANALYSTS, INC.

2450 HASSELL AOAO

WOFFMAN ESTATES, IL 60195

1847) $384-7377$

(350! $232-1907$

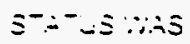

Abnorsa! in 29-MAR-95

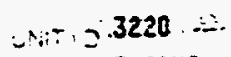

EZN:=ONJEN-ENG:NE

$\therefore=\because==\equiv=-\because 2491: ?$

\begin{tabular}{|c|c|c|}
\hline WCRKS: & $\begin{array}{l}\text { U.VT HANLFACTURER } \\
\text { INTN'L HARUESTER } 5000\end{array}$ & MOBIL DELUAC SAE 40 \\
\hline COUPONEN: :ソUE & CONIPONENTT HANUFACTLRER AND MODEL & 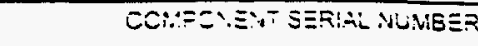 \\
\hline DIESEL ENGIKE & DETROIT DIESE: 6119? & $\$ A 32254$ \\
\hline
\end{tabular}

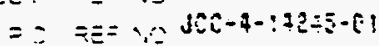

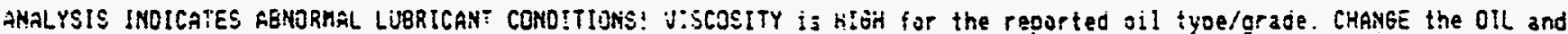
FilTERisi if not a'?ezoy performet. CHECK for proper operating temogratures. RESAFPLE at the next sthesulec intervel.

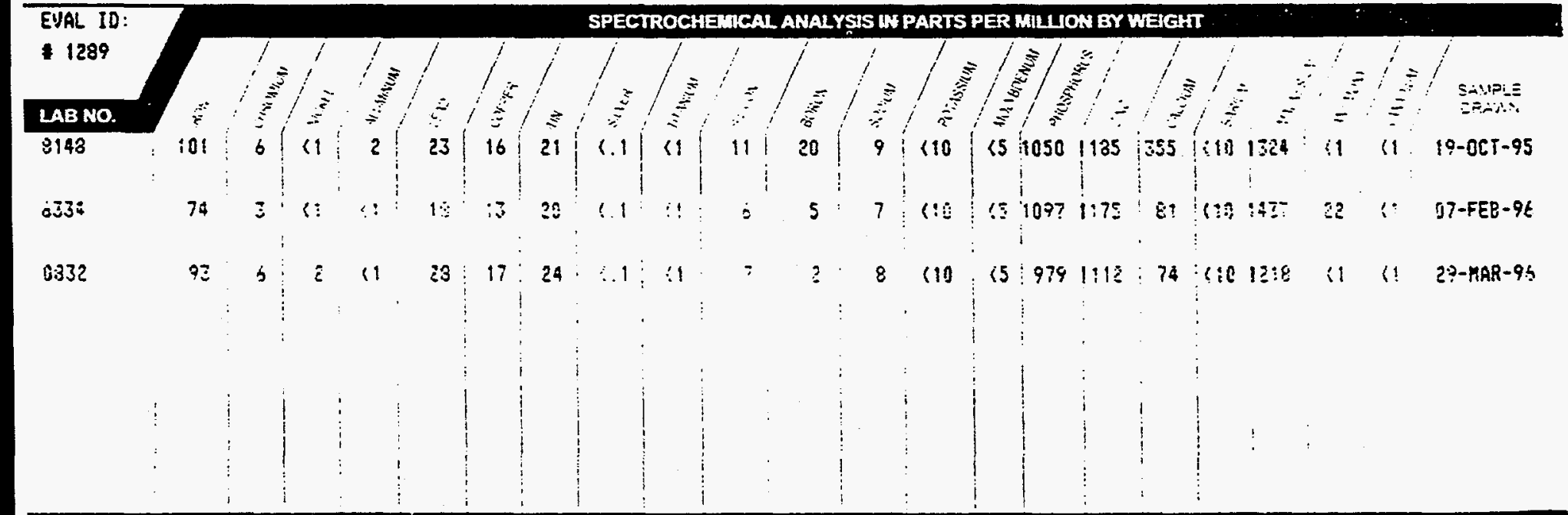

SAMPIE WFFORMATOW

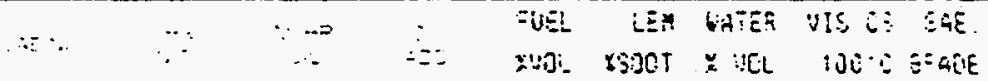

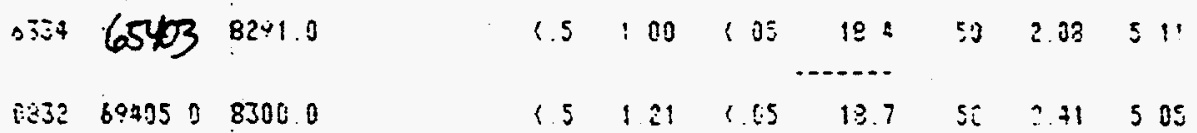


WENREP :K GOAKTY

J:A ALLEN

3:0 UESHINJTOM AUENLE

HERKINS AN 5EZAZ
ANALYSTS, INC. A

2450 HASSELL ROAD

HUFFMAN ESTATES, IL $60: 95$

(7L3) $884-757 ?$

(800; $222-00 ?:$

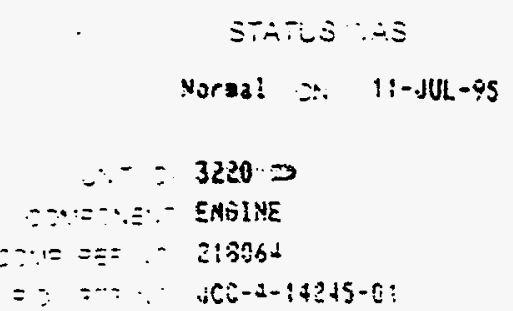

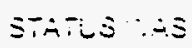

Noraz! $\because \quad 1:-J U L-95$

$\therefore \cdot== \pm=-21306$

HEMYEDIN UNIV

DIESEL ENG]HE $\because O F K \mathrm{I}^{-} \mathrm{E}$

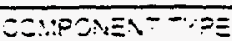

西
INTY: HARUESTER EGYSTAR SOCO

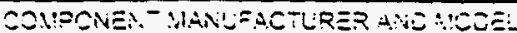
OEIROIT DIESEL $4-71$

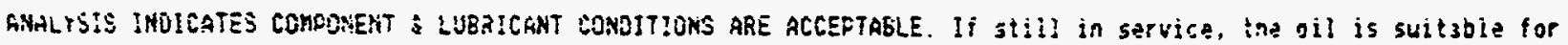
zontinied use. REEARFLE at the naxi jehesules irierva!.

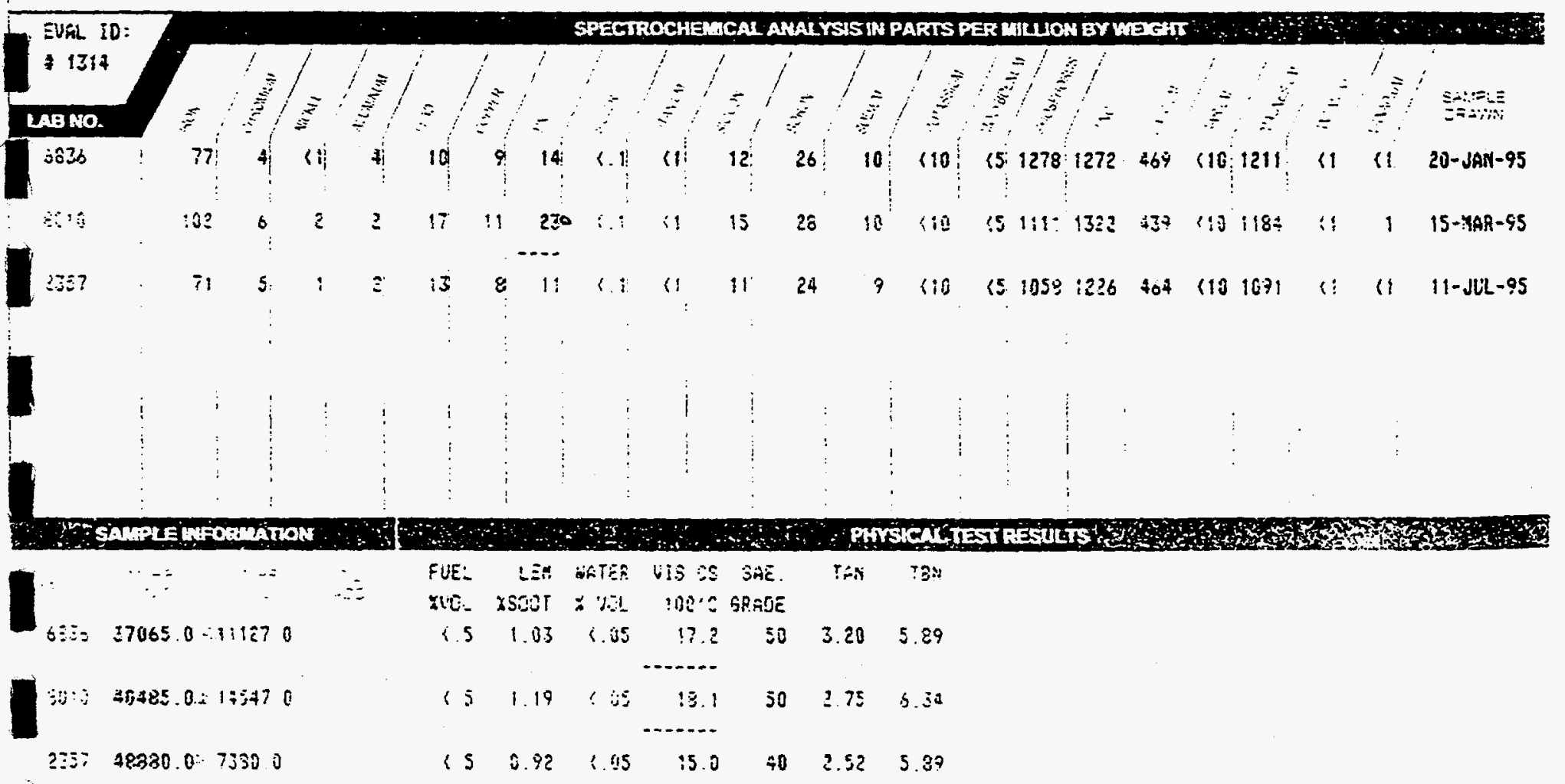


ZIEGLER OIL ANALYSIS LAB 901 WEST 94 TH STREET

MINNEAPOLIS, MN 55420.4299

(612) $888.4121 \cdot(800) 352.2812$

\section{$5 \cdot 0.5$}

SCHEDULED OIL SAMPLING WEAR ANALYSIS REPORT
Sample For
HENNEPIN CTY HIGHWAY DEPT JIM ALLEN

320 WASHINGTON AVENUE S HOPKINS MN 55343-8496
Serial \#:

Compartment: Diesel Engine

Unit \#: $\quad 3221$

Model \#:
Sample \#:

Date Received:

Oil Brand:

Oil Blend:

Oil Weight:
96094334

$9 / 23 / 96$

Caterpillar

Diesel Engine 0 $15 W 40$

DETROIT 6V92

\begin{tabular}{|c|c|c|c|c|c|c|c|c|c|c|c|c|c|c|c|c|c|c|c|c|c|}
\hline \multicolumn{6}{|c|}{ CURRENT SAMPLE INFORMATION } & \multicolumn{6}{|c|}{ WEAR METALS ANALYSIS } & \multicolumn{3}{|c|}{ 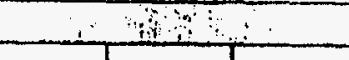 } & \multirow{2}{*}{\multicolumn{4}{|c|}{$\begin{array}{l}\text { INFAARED ANALYSIS } \\
\text { PERCENT OF ALLLOWABLE }\end{array}$}} & \multirow{2}{*}{\multicolumn{3}{|c|}{$\begin{array}{l}\text { PHYSICAL } \\
\text { TESTS }\end{array}$}} \\
\hline \multirow{2}{*}{$\begin{array}{l}\text { DATE } \\
\text { SAMPLE } \\
\text { TAKEN }\end{array}$} & \multicolumn{2}{|c|}{ CHANGED } & \multirow{2}{*}{$\begin{array}{l}\text { QUARTS } \\
\text { OIL } \\
\text { AODEO }\end{array}$} & \multirow{2}{*}{$\begin{array}{c}\text { HOUPS } \\
\text { ON } \\
\text { METEA }\end{array}$} & \multirow{2}{*}{$\begin{array}{l}\text { HOUAS } \\
\text { ON } \\
\text { Oll }\end{array}$} & \multirow{2}{*}{$\begin{array}{l}\text { CU } \\
\text { Coppor } \\
\text { PPM }\end{array}$} & \multirow{2}{*}{$\begin{array}{c}\text { FE } \\
\text { lion } \\
\text { PPM }\end{array}$} & \multirow{2}{*}{$\underset{\text { Chromilum }}{\text { PPM }}$} & \multirow{2}{*}{$\underset{\substack{\text { AL } \\
\text { Aluminum } \\
\text { PPM }}}{ }$} & \multirow{2}{*}{$\begin{array}{l}\text { SI } \\
\text { sitgon } \\
\text { PPM }\end{array}$} & \multirow{2}{*}{$\begin{array}{l}\text { PB } \\
\text { Lead } \\
\text { PPM }\end{array}$} & \multirow{2}{*}{$\begin{array}{l}\text { NA } \\
\text { Sodum } \\
\text { PPM }\end{array}$} & \multirow{2}{*}{$\begin{array}{c}\text { MO } \\
\text { Molybdenum } \\
\text { PPM }\end{array}$} & \multirow{2}{*}{$\begin{array}{c}\text { SN } \\
\text { Iin } \\
\text { PPM }\end{array}$} & & & & & & & \\
\hline & Oil & Filler & & & & & & & & & & & & & Soot & $\underset{\substack{\text { Sultation } \\
*}}{ }$ & Oxidation & $\begin{array}{l}\text { Nintation } \\
\%\end{array}$ & $\begin{array}{l}\text { Anti- } \\
\text { Freazo }\end{array}$ & $\mid \begin{array}{c}\text { Fuel } \\
\text { Dilulion }\end{array}$ & Walor \\
\hline $9 / 19 / 96$ & U & U & & 60715 & 15625 & 65 & 39 & 1 & 1 & 10 & 92 & 251 & 1 & 12 & 23 & 7 & 4 & 1 & $\mathbf{P}$ & $\mathbf{N}$ & $\overline{\mathrm{T}}$ \\
\hline
\end{tabular}

Interpretation

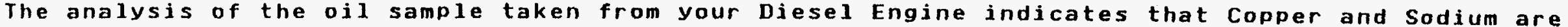
high; Lead is extremely high. This oil sample tests positive for water and antifreeze contamination. $0 i 1$ sample has a trace of water contamination.

Recommendation

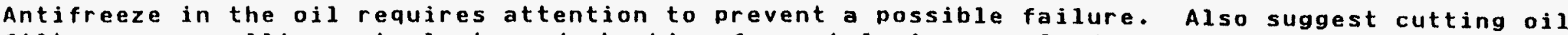
filter open. pulling out pleats and checking for metal pieces. Condensation possible cause of water in this compartment, oil may not be warm enough to evaporate moisture.

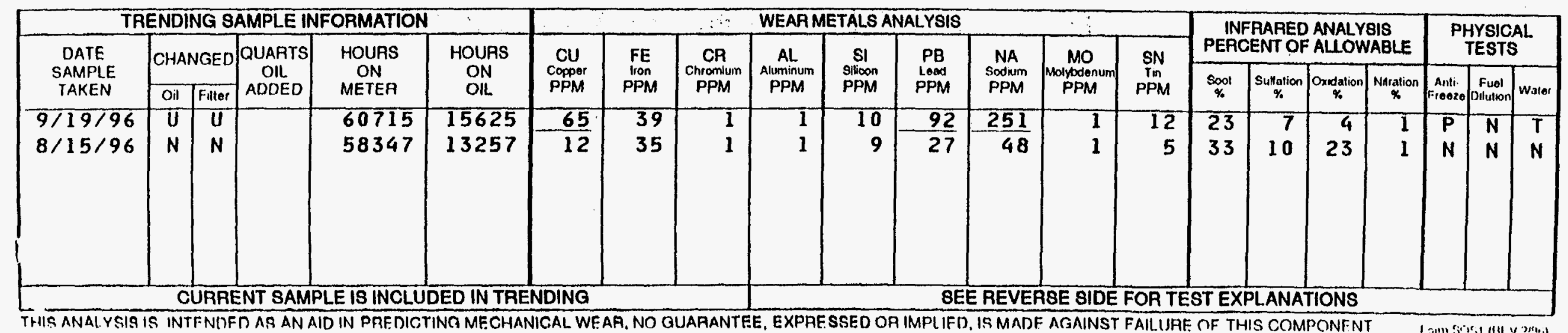


TENHEFIN COLNT:

J!H A-LEI

32.) JASHINGTCN HUENIJE

rijexills mit $55 ; 45$

\section{ANALYSTS, INC. A}

2450 HASSELL SCFB

HSFEMHA ESTATES, IL SOIYJ

17381 $385-797 ?$

(300) 22?-.30?:

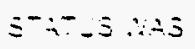

Yeraz! $\because \therefore \quad 21-J U L-95$

$\therefore \because \quad 3221$

$\because \because \because \because: \because$ ENS! RE

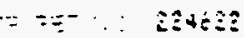

ง $60-7-14245-81$

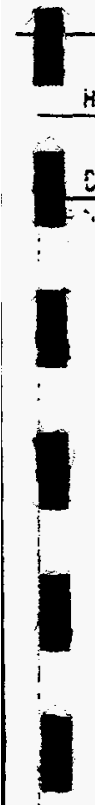

HEMNEFIH COUNT:

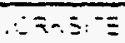

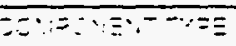

CIESEL ENGIME

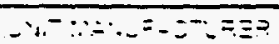

INTA'L HARUESTER JATETAR SODE

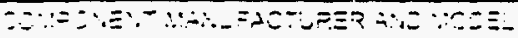

CEFREIT OIESEL GUYT2

\section{$\therefore-\cdots$}

MOE I: OELUEC SAE 40

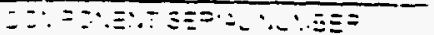

QSUF 200396

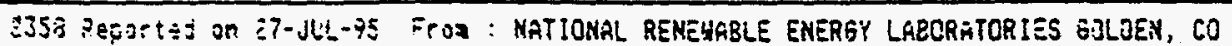

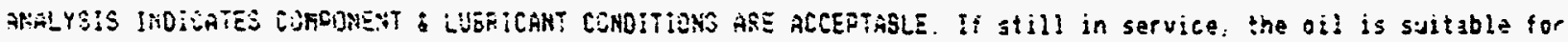

orritirued die RESARELE at ins neat scheduite interJal

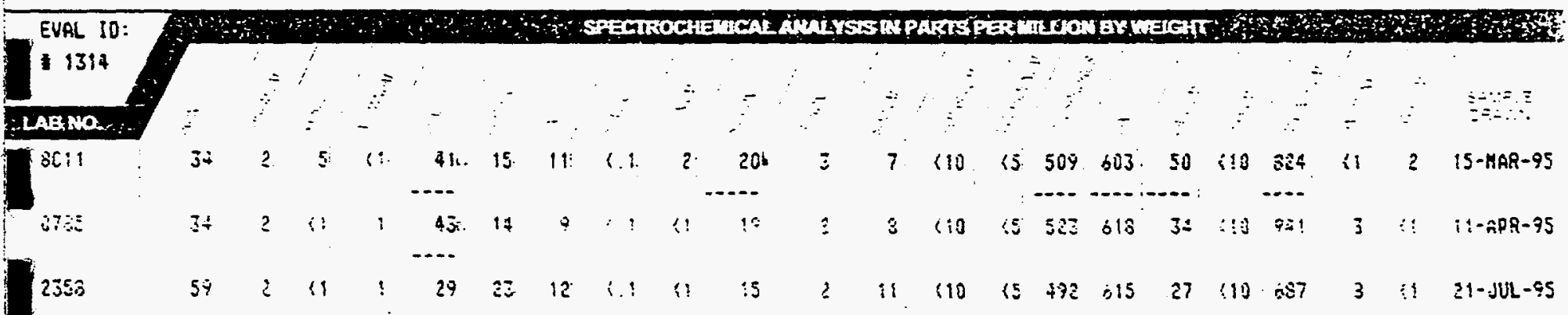

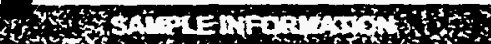

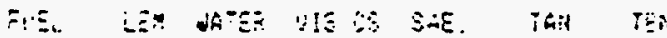

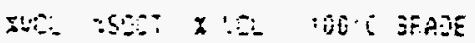

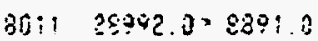

, 5 o 04 is $158 \quad 43 \quad 2.52 \quad 2.3 ?$

23528869 Bs:

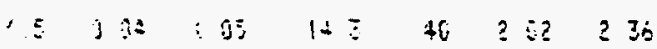

$235535351.0,5072=$

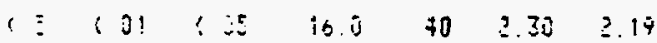


SCHEDULED OIL SAMPLING WEAR ANALYSIS REPORT

Sample For:
Serial \#:

Compartment:

Unit \#:

Model \#:
$06 V 200397$

Diesel Engine 3228

$\begin{array}{ll}\text { Sample \#: } & 96103208 \\ \text { Dale Received: } & 10 / 15 / 96 \\ \text { Oil Brand: } & \text { She11 } \\ \text { Oil Blend: } & \text { Rotella T } \\ \text { Oil Weight: } & 15 \text { W O }\end{array}$

\begin{tabular}{|c|c|c|c|c|c|c|c|c|c|c|c|c|c|c|c|c|c|c|c|c|c|}
\hline \multicolumn{6}{|c|}{ CURRENT SAMPLE INFORMATION } & \multicolumn{9}{|c|}{ WEAA METALS ANALYSIS } & \multirow{2}{*}{\multicolumn{4}{|c|}{$\begin{array}{c}\text { INFAAARED ANALYSIS } \\
\text { PERCENT OF ALLOWABLE }\end{array}$}} & \multirow{2}{*}{\multicolumn{3}{|c|}{$\begin{array}{l}\text { PHYSICAL } \\
\text { TESTS }\end{array}$}} \\
\hline DATE & $\mathrm{CH}$ & IGED & QUARTS & HOURS & HOU & & & & & & & & & & & & & & & & \\
\hline $\begin{array}{l}\text { SAMPLE } \\
\text { TAKEN }\end{array}$ & Oil & Filler & $\begin{array}{c}\text { Olt. } \\
\text { ADDED }\end{array}$ & $\begin{array}{c}\text { ON } \\
\text { METEA }\end{array}$ & $\begin{array}{l}\text { ON } \\
\text { OlL }\end{array}$ & $\begin{array}{l}\text { Copper } \\
\text { PPM }\end{array}$ & $\begin{array}{l}\text { lon } \\
\text { PPM }\end{array}$ & Chromium & $\begin{array}{l}\text { Aluminum } \\
\text { PPM }\end{array}$ & PP & PPM & $\begin{array}{l}\text { Solum } \\
\text { PPM }\end{array}$ & PPM & PPM & $\begin{array}{l}\text { Soot } \\
\%\end{array}$ & $\begin{array}{c}\text { Sulfation } \\
\%\end{array}$ & Oxidaition & $\underset{\%}{\text { Niration }}$ & $\begin{array}{l}\text { Antl. } \\
\text { Freoze }\end{array}$ & Fuel & \\
\hline $0 / 03 / 96$ & $\mathbf{N}$ & $\mathrm{N}$ & & 32803 & 5559 & 24 & 47 & 2 & 1 & 10 & 20 & 38 & 4 & 10 & 40 & 25 & 29 & 16 & $N$ & $\mathbf{N}$ & $N$ \\
\hline
\end{tabular}

Interpretation

The analysis of the oil sample taken from your Diesel Engine indicates that copper and Lead are

slightly high.

Recommendation

Continue to monitor compartment by sampling at recommended intervals.

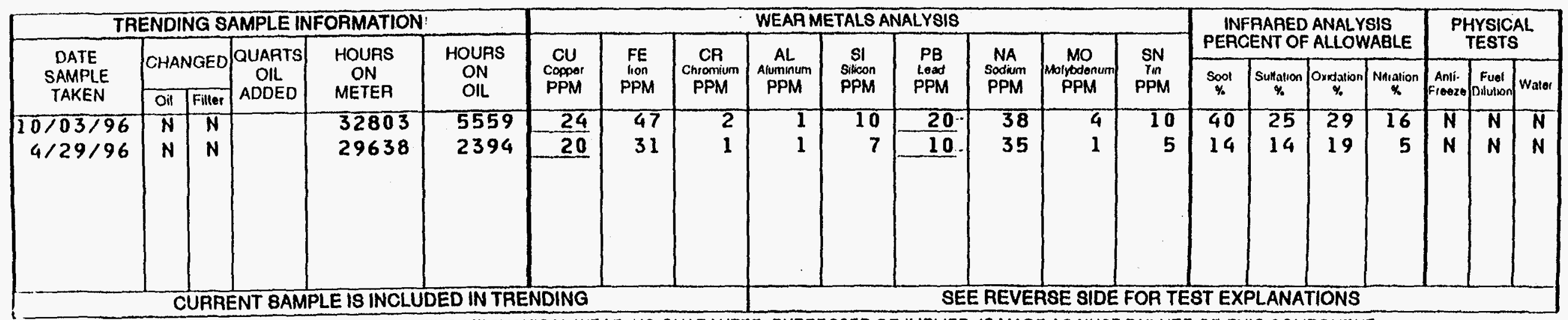


HEANEETN SOUNTY

JI: ALLEN

?.) YUS?INGTON RUENUE

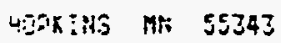

ANALYSTS, INC.

2450 HASSELL ROAO

MCFFMAN ESTATES, IL 60195

(847) $884-7977$

(300) $222-0071$
STATUS WAS

Abnorad ON 08-FE8-96

UNIT: 0.3228

COMPONENT ENGINE

CONP FE= NO 249114

$=0$ : SEF NO JCL-4-14245-01

\begin{tabular}{|c|c|c|}
\hline WORKSITE & $\begin{array}{l}\text { UNIT WANUFACTLRER } \\
\text { INTK'L HARUESTER PAYSTAR SOO }\end{array}$ & $\begin{array}{l}\text { OIL TYPE } \\
\text { MOBIL OELUAC SAE } 40\end{array}$ \\
\hline $\begin{array}{l}\text { CONPONENT TYPE } \\
\text { FLCOHOL FIJELEO ENGIME }\end{array}$ & $\begin{array}{l}\text { COMPONENT MANUFACTURER AND ANODEL } \\
\text { DETROIT OIESEL GU92 }\end{array}$ & $\begin{array}{l}\text { COMPONENT SERIAL NUMBEF } \\
\text { O6UF200397 }\end{array}$ \\
\hline
\end{tabular}

ANALYSIS INDICATES ABNOFMAL COMPONENT CONDITIONS! Bearing overlay wear. CHANGE the OIL and FILIER(s) if not already perforaed. CHECK for proper start-up and operating oil pressures. RESAKPLE at $1 / 2$ noraal interval.

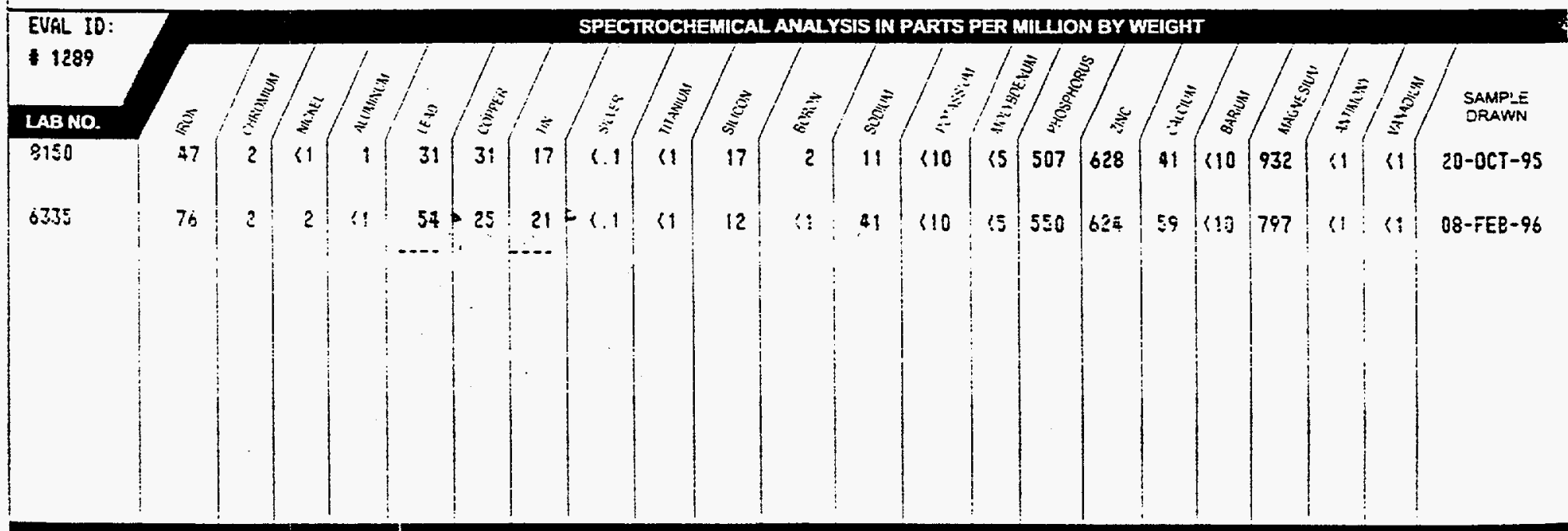

SAMPLE WFFORATION

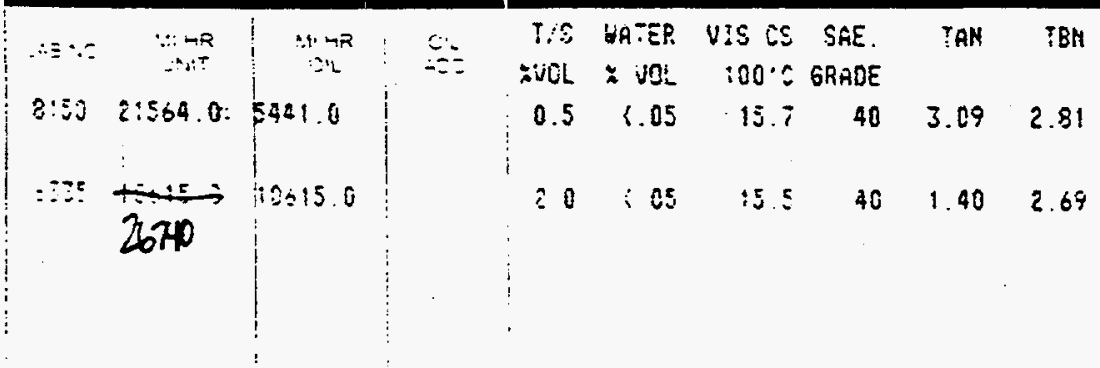




\section{Appendix 7 \\ Summary of Emissions Testing}




\section{Emissions Testing of Hennepin County Snowplows \\ Nigel Clark, WVU, October 2, 1997}

Two ethanol powered and one diesel powered snowplows were subjected to emissions testing by the West Virginia University Transportable Heavy-Duty Emissions Testing Laboratories. The Transportable Laboratories were originally constructed to satisfy the need to gather data on emissions from heavy-duty vehicles without the need to remove engines from the vehicles for testing. The laboratories are transportable to permit testing at the site of truck operation and to ensure that tested vehicles were out of service for as short a time as possible. The laboratory facility arrives on the test site pulled on two trailers, one being a box trailer containing equipment for emissions measurement, data acquisition and control, and the other, a flat bed semi-trailer carrying the power absorber unit. The flat bed is lowered to the ground to provide a chassis dynamometer platform.

The vehicle to be tested is driven onto the flat bed and the wheels of the vehicle are positioned on rollers set in the bed. The outer wheels of the dual wheel set on each side of the vehicle are connected to the drive shafts of the dynamometer units located on each side of the vehicle. Each dynamometer unit consists of speed increasing gearboxes with a power absorber and a flywheel set. The flywheel sets consist of a series of selectable discs to allow simulation of vehicle inertia. During the test cycle, torque cells and speed transducers in the power absorber drive train measure the actual vehicle load and speed.

The full exhaust from the tail pipe of the test vehicle was ducted to a $45 \mathrm{~cm}$ diameter dilution tunnel on top of the emissions trailer. The exhaust was mixed with dilution air and the flow was controlled using a blower with critical flow venturis. Heated sampling probes sent diluted exhaust to a number of different gas analysis instruments, via heated lines. Levels of carbon dioxide $\left(\mathrm{CO}_{2}\right)$, carbon monoxide $(\mathrm{CO})$, oxides of nitrogen $\left(\mathrm{NO}_{\mathrm{x}}\right)$, and hydrocarbons $(\mathrm{HC})$ were measured continuously. A bulk measurement of particulate matter (PM) was obtained using 70 $\mathrm{mm}$ filters weighed after conditioning for temperature and humidity in an environmental chamber. Levels of alcohol and aldehydes were determined using impingers and cartridges, respectively, with subsequent laboratory analysis.

The Hennepin County snowplows had 10-speed unsynchronized transmissions and as such would have had difficulty in following the Central Business District speed-time test schedule that is commonly used for automatic transit buses. The bulk of the testing was therefore undertaken using the West Virginia University 5-peak truck cycle, which has a total distance of 5 miles, a time of 900 seconds, and five peaks, at 20,25,30,35, and 40 miles per hour.

The plows were tested in March and April of 1994, and again in May of 1995. Table 1 provides the vehicle mileage at the time of testing.

Table 2 provides emissions data gathered in 1994, while Table 3 provides 1995 initial and repeat run data.

The hydrocarbons reported for the vehicles are direct readings from the flame ionization detector 
(FID) of the HC analyzer, which is calibrated using propane. A more representative indication of the hydrocarbon emissions for ethanol vehicles is often expressed as the "organic material hydrocarbon equivalent" (OMHCE), which is calculated as follows to correct for species and to exclude weight of oxygen in the species, with suitable background corrections.

$\mathrm{OMHCE}=(\mathrm{FIDHC})-0.768\left(\mathrm{CH}_{3} \mathrm{OH}\right)+0.4621(\mathrm{HCHO})=0.6298\left(\mathrm{CH}_{3} \mathrm{CHO}\right)+0.6023\left(\mathrm{C}_{2} \mathrm{H}_{5} \mathrm{OH}\right)$,

where $\mathrm{C}_{2} \mathrm{H}_{5} \mathrm{OH}$ is ethanol, $\mathrm{HCHO}$ is formaldehyde, $\mathrm{CH}_{3} \mathrm{CHO}$ is acetaldehyde, and $\mathrm{CH}_{3} \mathrm{OH}$ is methanol. In essence, the FID reading is corrected for the calibration with respect to ethanol $\left(\mathrm{C}_{2} \mathrm{H}_{5} \mathrm{OH}\right)$, which is independently measured using impingers, and is increased by the level of formaldehyde $(\mathrm{HCHO})$ and acetaldehyde $\left(\mathrm{CH}_{3} \mathrm{CHO}\right)$ measured using cartridges, since these two species do not register with the FID.

The data generally show that oxides of nitrogen are slightly lower for the ethanol vehicles than the diesel vehicle, and that particulate matter levels are significantly lower for ethanol. The diesel emissions levels remained consistent from year to year, and offered lower carbon monoxide emissions than the ethanol trucks, whereas the carbon monoxide emissions from one vehicle (3228) rose by a factor of three between 1994 and 1995. It is possible that catalytic converter efficiency had degraded on this vehicle, because hydrocarbon emissions also rose. The diesel vehicle had lower hydrocarbon emissions than the ethanol vehicles and it was evident that unburned ethanol was present in the exhaust of the ethanol vehicles as a significant fraction of the total hydrocarbon emissions. However, since oxides of nitrogen and particulate matter may be considered the emissions of concern for the heavy-duty fleet, one must conclude that the ethanol powered vehicles offered a solution to emissions inventory reduction.

Table 1. Mileage as Received for the Three Snowplows

\begin{tabular}{|l|l|l|}
\hline Unit No. & $\mathbf{1 9 9 4}$ & $\mathbf{1 9 9 5}$ \\
\hline HC - 3221 Ethanol & 8842 & 30,353 \\
\hline HC - 3228 Ethanol & 5175 & 17,170 \\
\hline HC - 3220 Diesel & 12,409 & 43,806 \\
\hline
\end{tabular}


Table 2. Data from 5-Peak Cycle Runs Gathered in 1994

\begin{tabular}{|l|l|l|l|l|l|l|l|l|l|}
\hline $\begin{array}{l}\text { WVU } \\
\text { Sequence \# }\end{array}$ & Unit & $\mathrm{CO}$ & $\mathrm{NO}_{2}$ & PM & FIDHC & OMHCE & $\mathrm{HCHO}$ & $\mathrm{C}_{2} \mathrm{H}_{5} \mathrm{OH}$ & $\mathrm{CH}_{3} \mathrm{CHO}$ \\
\hline 261 & $\begin{array}{l}\text { HC-3221 } \\
\text { Ethanol }\end{array}$ & 7.4 & 12.3 & 0.30 & 4.98 & 4.33 & 0.11 & 2.41 & 0.56 \\
\hline 259 & $\begin{array}{l}\text { HC-3228 } \\
\text { Ethanol }\end{array}$ & 9.0 & 14.8 & 0.45 & 5.69 & 5.21 & 0.15 & 3.37 & 0.66 \\
\hline 258 & $\begin{array}{l}\text { HC-3220 } \\
\text { Diesel }\end{array}$ & 4.3 & 13.2 & 0.83 & 1.35 & -- & --- & -- & --- \\
\hline
\end{tabular}

Table 3. Data from 5-Peak Cycle Runs Gathered in 1995

\begin{tabular}{|l|l|l|l|l|l|l|l|l|l|}
\hline $\begin{array}{l}\text { WVU } \\
\text { Sequence } \\
\#\end{array}$ & Unit & $\mathrm{CO}$ & $\mathrm{NO}_{2}$ & $\mathrm{PM}$ & $\mathrm{FIDHC}$ & $\mathrm{OMHCE}$ & $\mathrm{HCHO}$ & $\mathrm{C}_{2} \mathrm{H}_{5} \mathrm{OH}$ & $\mathrm{CH}_{3} \mathrm{CHO}$ \\
\hline 431 & $\begin{array}{l}\text { HC- } \\
3221 \text { Ethanol }\end{array}$ & 5.1 & 11.8 & 0.35 & 3.86 & 2.81 & 0.08 & 0.62 & 0.43 \\
\hline 434 & $\begin{array}{l}\text { HC-3228 } \\
\text { Ethanol }\end{array}$ & 26.2 & 12.0 & 0.56 & 7.07 & 6.73 & 0.29 & 3.74 & 1.88 \\
\hline 436 & $\begin{array}{l}\text { HC-3220 } \\
\text { Diesel }\end{array}$ & 4.6 & 13.3 & 0.85 & 1.50 & --- & --- & -- & -- \\
\hline 453 & $\begin{array}{l}\text { HC-3221 } \\
\text { Repeat }\end{array}$ & 7.7 & 11.3 & 0.39 & 4.02 & 2.88 & 0.08 & 0.74 & 0.54 \\
\hline 454 & $\begin{array}{l}\text { HC-3228 } \\
\text { Repeat }\end{array}$ & 31.2 & 11.7 & 0.56 & 7.17 & 6.86 & 0.26 & 4.33 & 1.57 \\
\hline 460 & $\begin{array}{l}\text { HC-3220 } \\
\text { Repeat }\end{array}$ & 5.8 & 13.0 & 0.88 & 1.38 & --- & --- & --- & -- \\
\hline
\end{tabular}




\section{Appendix 8 \\ Details of Emissions Testing}




\section{WEST VIRGINIA UNIVERSITY \\ Transportable Heavy Duty Vehicle Emissions Testing Laboratory}

\section{Test Results Report}

Test Date: $\quad$ March, 1994 and May, 1995

Test Vehicles: Snow Plow 3220, 3221 and 3228

Fuel Type: $\quad$ E100 and Diesel

Engine Type: DDC 6V-92TA

\section{Submitted to Hennepin County}

Department of Mechanical \& Aerospace Engineering West Virginia University

Morgantown, WVV 26506-6106

Phone: (304) 293-3111

January 28, 1996 


\section{Contents}

Part I. Test Driving Cycles

Part II. Test Circumstances

Part III. Test Results

Part IV. Emissions Comparison of Ethanol vs. Diesel 


\section{Part I}

\section{Test Driving Cycles}

Three cycles were used for the tests: CBD cycle and Truck-CBD cycle and WVU-Truck cycle. The descriptions of the Cycles are as follows:

\section{Cycle Name}

CBD

Truck-CBD

WVU-Truck

\section{Descriptions of Test Cycles}

It is abbreviated from the Central Business District (CBD) phase of the Transit Coach Design Operation Profile Duty Cycle. It was developed by the Federal Transit Administration (FTA) and it was included in SAE Recommended Practice. SAE J1376 JUL82.

The driving pattern in this cycle is a general representation of transit vehicle operation in a downtown business district. The cycle consists of fourteen identical segments. Each segment includes 10 seconds of acceleration, 18.5 seconds of $20 \mathrm{mph}$ cruise. 4.5 seconds of deceleration and 7 seconds of idle. The total driving distance is 2 miles.

This cycle was modificd from the Central Business District (CBD) cycle and was designed for heary-duty truck testing. It was developed by West Virginia University facuity in 1992.

The driving pattem in this cycle is a general representation of trucks operating in a downtown business distriet. The cycle consists of fourteen identical segments. The maximum speed of this cycle is $20 \mathrm{mph}$. The total duration of this cycle is 850 seconds. Each segment includes 30 seconds of acceleration, 5 seconds of $20 \mathrm{mph}$ cruise, 15 seconds of deceleration and 10 seconds of idle. The total driving distance is 2.18 miles.

It is abbreviated from the West Virginia University Truck cycle. It was developed by West Virginia Liniversity faculty in 1994. More information can be found in SAE paper 951016.

This cycle is designed for general truck chassis testing. The cycle consists of five segments. Each segment includes an acceleration to a certain speed and then keep this speed for a penod and then a deceleration to idle. The acceleration and deceleration are constants for all 5 segments. The five constant speeds for the five segments are 20, 25. 30, 35. and $40 \mathrm{mph}$. The duration of this cycle is 900 seconds and the total driving distance is 5 miles. 


\section{CBD Cycle}

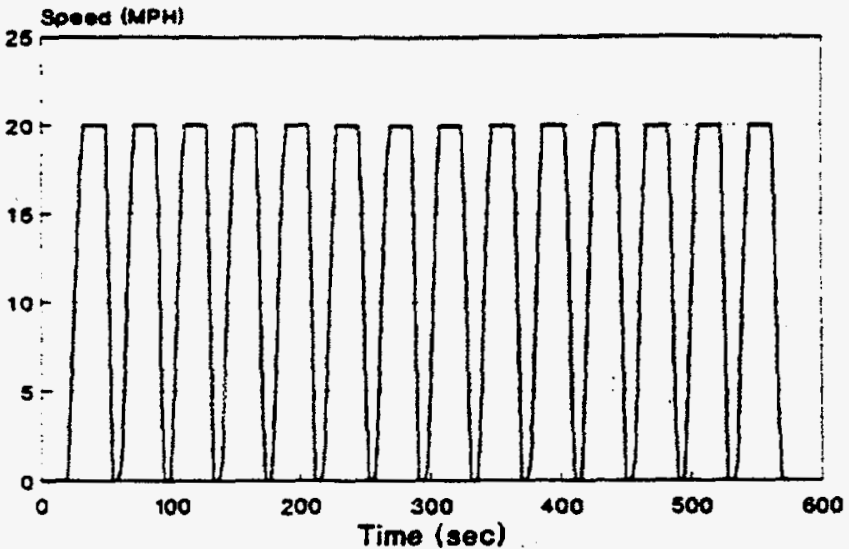

\section{Truck-CBD Cycle}

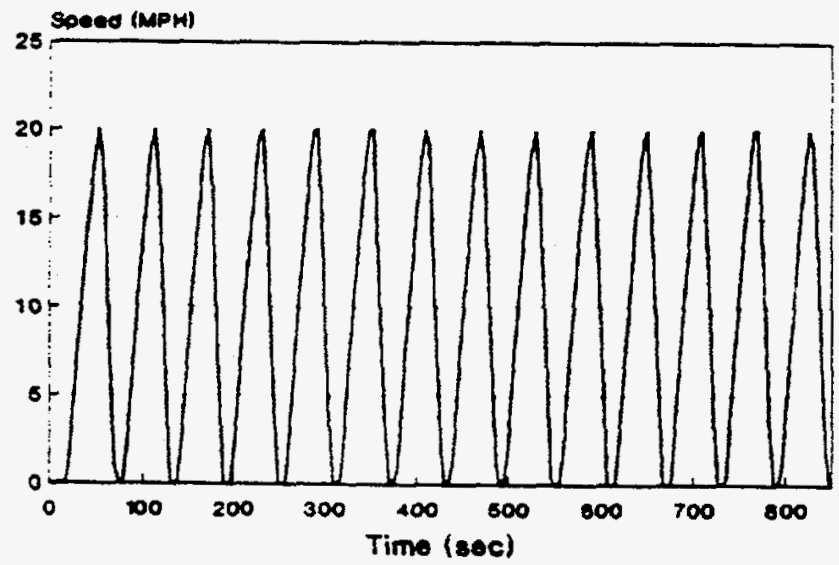

\section{WVU-Truck Cycle \\ (5-Peak Truck Cycle)}

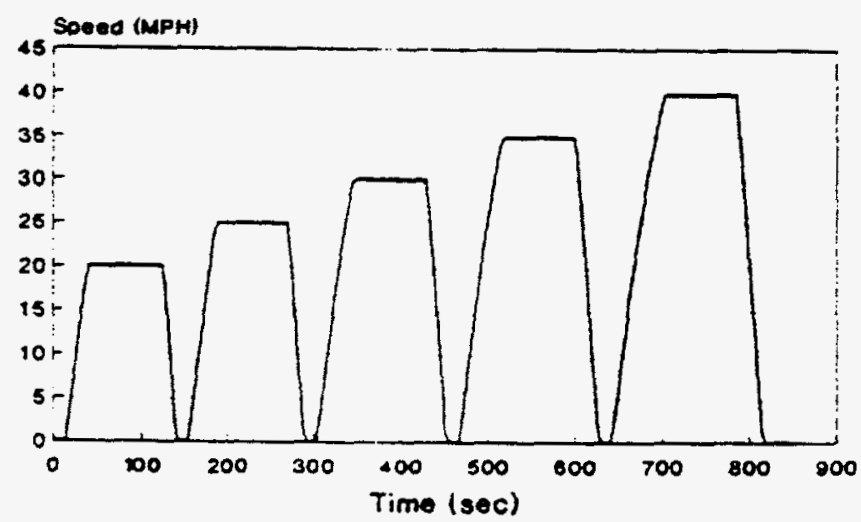




\section{Part II}

\section{Test Circumstances}

Eighteen tests were conducted for Hennepin County trucks. In order to obtain vehicle emissions data with various driving conditions, Each vehicle was tested with three driving cycles. The following tables show the general information of each test.

1.Tests for Truck 3220

\begin{tabular}{|c|l|c|c|c|c|}
\hline Test 1D & WVU Reforence Number & Test Cycie & Test Dote & Fuel & Vohiclo Model Year \\
\hline 257 & HC-3220-JA & CBD & $03 / 28 / 94$ & Diesel & 1993 \\
\hline 435 & HC-3220-D1-TCBD & TruCK-CBD & $05 / 10 / 95$ & Diesel & 1993 \\
\hline & & & & & \\
\hline 248 & HC-3220-D2 & WVU-Truck & $03 / 18 / 94$ & Diesel & 1993 \\
\hline 258 & HC-3220-JA-TRK & WVU-Truck & $03 / 28 / 94$ & Diesel & 1993 \\
\hline 436 & HC-3220-D1-TRK & WVU-Truck & $05 / 09 / 95$ & Diesel & 1993 \\
\hline 453 & HC-3220-D1-TRK-R & WVU-Truck & $05 / 20 / 95$ & Diesel & 1993 \\
\hline
\end{tabular}

2.Tests for Truck 3221

\begin{tabular}{|c|c|c|c|c|c|}
\hline Test 1D & WvU Reference Number & Test Cycle & Test Date & Fuel & Vehich Model Yeal \\
\hline 262 & HC.3221-E100 & $C B D$ & $03 / 30 / 94$ & Ethanol & 1993 \\
\hline \multirow[t]{2}{*}{432} & HC-3221-E100-TCBD & Truck. CBO & $05: 05 / 95$ & Ethanol & 1993 \\
\hline & & & 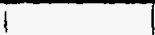 & & \\
\hline 250 & HC-3221-E100 & WvU-Truex & $103 / 19 / 94$ & Ethenol & 1993 \\
\hline 261 & HC-3221-E100-TRK & WVU.Truck & $103 / 30 / 94$ & Ethanol & 1993 \\
\hline 431 & HC-3221-E100-TRK & WVU-Truck & $05 / 05 / 95$ & Ethanol & 1993 \\
\hline 454 & HC-3221-E100-TRK-R & WVU-Truek & $05 / 21 / 95$ & Ethanol & 1993 \\
\hline
\end{tabular}

3.Tests for Truck 3228

\begin{tabular}{|c|c|c|c|c|c|}
\hline Tost ID & WVU Reference Number & Tesi Crale & Test Date & Fuel & Vehicle Model Year \\
\hline 260 & HC-3228-E 100 & CBO & $03 / 29 / 94$ & Ethanol & 1993 \\
\hline 433 & IHC-3228-E10O-TCBD & Truck-CBO & $05 / 06 / 95$ & Ethanol & 1993 \\
\hline & & & & & \\
\hline 251 & $\mathrm{HC}-3228 \cdot E 100$ & NVU Truck & $03 / 21 ; 94$ & Ethanol & 1993 \\
\hline 259 & IHC-3228-EIOO-TRK & WVU.Truck & $03 / 29 / 94$ & Ethanol & 1993 \\
\hline 434 & HC-3228-E100-TRK & WVU-Truek & $105 / 06 / 95$ & Ethanol & 1993 \\
\hline 460 & HC-3228-E10O-TRK-R & :NVU.Truck & $05 / 23 / 95$ & Ethanol & 1993 \\
\hline
\end{tabular}




\section{Part III}

\section{Test Results}

Eighteen sheets of the test results are included in this part. This contains vehicle and engine information, vehicle emissions rates and fuel economy of each individual run. Explanations of the emissions constituents are also included. The WVU Transportable Heavy-Duty Vehicle Emissions Testing Laboratory can simulate the on-road operation of the tested vehicle. The trucks driven on the chassis dynamometer were under the actual driving conditions which include the vehicle's inertial resistance, tire rolling resistance and air resistance on road. The actual driving speed was measured and the driving distance of each test run was calculated. Emissions data were based on 10-hz continuous measurements during the test runs. The mass of each pollutant was calculated from integration of the instantaneous concentration over the whole test cycle. The reported emissions rate is the emissions constituent mass over the actual driving distance of the test run.

The test results are the summary of the data obtained from several repeat runs conducted for each test set-up. 


\title{
Description of component codes
}

\author{
CO: $\quad$ carbon monoxide \\ $\mathrm{CO}_{2}$ : carbon dioxide \\ $\mathrm{NO}_{\mathrm{z}}: \quad$ oxides of nitrogen \\ FIDHC: total hydrocarbon measured by HFID. For CNG vehicles, unburned methane is included. \\ For ajcohol vehicles, unburned methanol or ethanol and formaldehyde are included. \\ PM: particulate matter \\ $\mathrm{CH}_{3} \mathrm{OH}$ : unburned methanol \\ $\mathrm{C}_{3} \mathrm{H}_{5} \mathrm{OH}$ : unburned ethanol \\ HCHO: formaldehyde \\ $\mathrm{CH}_{3} \mathrm{CHO}$ : aceraldehyde \\ RHC: calculated residual hydrocarbon for alcohol fueled vehicies. The amount (in gram/mile) of \\ $\mathrm{RHC}$ can be calculated from the concentration (in ppm) of $\mathrm{C}_{\mathrm{RHC}}$ which is obtained from the \\ following equation:

$$
\begin{aligned}
& C_{\mathrm{RHC}}=C_{\mathrm{FDHC}}-r_{1} \times C_{\mathrm{CH} 3 \mathrm{OH}} \text { for methanol or } \\
& C_{\mathrm{RHC}}=C_{\mathrm{FDHC}}-r_{2} \times C_{\mathrm{CZHSOH}}-r_{3} \times C_{\mathrm{CH} 3 \mathrm{CHO}} \text { for ethanol }
\end{aligned}
$$ \\ $\mathrm{C}_{\text {FDHC }}$ represents the measured concentration (in ppm) by Heated FID \\ $r_{1}, r_{2}, r_{3}$ are the HFWD response factors to methanol. ethanol and acetaldehyde, \\ respectiveiy. For the WVU lab. \\ $r_{1}=0.73, r_{2}=0.73, r_{3}=0.473$ from the HFD calibration \\ $\mathrm{C}_{\mathrm{CH} 3 \mathrm{OH}}$ and $\mathrm{C}_{\mathrm{CZHSOH}}$ represent the measured concentration (in ppm) of methanol and \\ ethanol. respectively.
}

OMHCE: calculated amount (in gram/mile) of organic material hydrocarbon equivalent. Two different equations with methanol and ethanol are used as following:

For methanol:

$$
O M H C E=R H C+\frac{13.8756}{32.042} \times \text { Meth }_{\text {mas }}+\frac{13.8756}{30.0262} \times \text { Form }_{\text {maxs }}
$$

For ethanol:

$$
O M H C E=R H C+\frac{13.8756}{23.034} \times E t_{\text {mass }}+\frac{13.8756}{30.0262} \times \text { Form }_{\text {mass }}+\frac{13.8756}{22.03} \times \text { Acet }_{\text {mass }}
$$

where. Meth $h_{\text {mass: }}$ unburned methanol emissions in gram/mile:

Ethmass: unburned ethanol emissions in gram/mile:

Form mass: unburned formaldehyde emissions in gram/mile:

Acet $t_{\text {mass: }}$ unburned acetaldehyde emissions in gram/mile.

MPG: calculated fuel economy (in mile/gallon).

BTU: calculated fuel energy used by the vehicie. 
Test Sequence Number: 257

WVU Test Reference Number

Fied Owner Full Name

Fieed Address:

Fieet Address (City, State, Zip Code)

Vehicte Type

Vehick ID Number (VIN)

Vehiele Manufacturer

Vehicie Model Year

Gross Vehicie Weight (GWM) (Ib)

Vehicie Total Curb Weight (lb)

Vehicie Simulated Weight (lb)

Odorneter Reading (mite)

Vehikde Transmission Type

Vehicie Transmiscion Configuration

Number Of Ades

\section{HC-3220-JA}

Engine Type

Engine 10 Number

Engine Displacement (liter)

Number Of Cylinders

Engine Rated Power (hp)

Hennepin County

320 Washington Ave.

Hoploins, MN 55343

Primary Fuel

Primary Fuet Sample ID

Snow Plow

2HTTGDVT1PCOBS272

NVSTR

1993

55020

27510

38568

Not Available

Manual

Fuller RTX-11708LL 10-Spd

3

DDC SV-92TA DDEC II

OSVF199374

9.05

6

277

JETA

N-WV-1152

Not Applicable

Not Applicable

Secondary Fuei Sample iD

Not Applicable

Particulate Trap Manufacturer

Not Applicable

Test Cycle

CBD

Test Date

$3 / 28 / 94$

Emissions Results (g/mile)

Fuel Economy

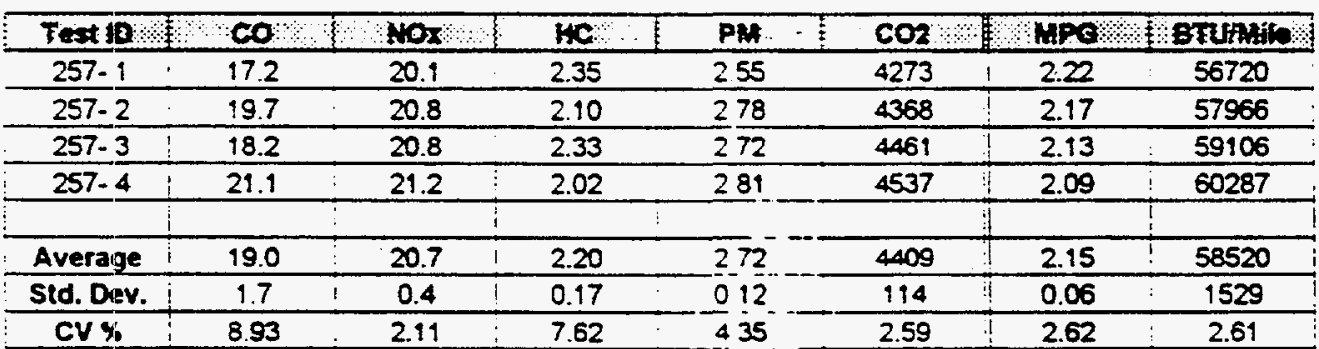


Test Sequence Number: 435

WVU Test Reference Number

Fled Owner Fut Name

Fleet Addrese

Fieet Address (City, State, Zip Code)

Vehicie Type

Vehicie ID Number (VIN)

Vehicie Manufacturer

Vehicie Model Year

Grose Vehicie Weight (GWM) (lb)

Vehicie Total Curb Weight (lb)

Vehicte Simuleted Weight (lb)

Odometer Reading (mile)

Vehicle Transmission Type

Vehicte Transmiscion Configuration

Number Of Ades

HC-3220-D1-TCBD

Engine Type

Engine ID Number

Engine Displacement (liter)

Number Of Cyinders

Engine Rated Power (hp)

Hennepin Courny

320 Washington Ave.

Hopkins, MN 55343

Snow Plow

2HTTGDVTIPCOS9272

NVSTR

1993

55020

27510

38575

43806

Manuat

Fuller RTX-11708LL 10-Spd

DDC 6V-92TA DDEC II

O6VF199374

9.05

6

277

Primary Fued

Primary Fuel Sample 10

Secondary Fue

Secondary Fuet Sample ID

Particutate Trap Manutacturer

Catalytic Converter Manufacture

D1

95-04

Not Applicable

Not Applicable

Not Appticable

Not Applicable

Test Cycle

Truek-CBD

Test Date

5/10/95

Emissions Results (g/mile)

Fuel Economy

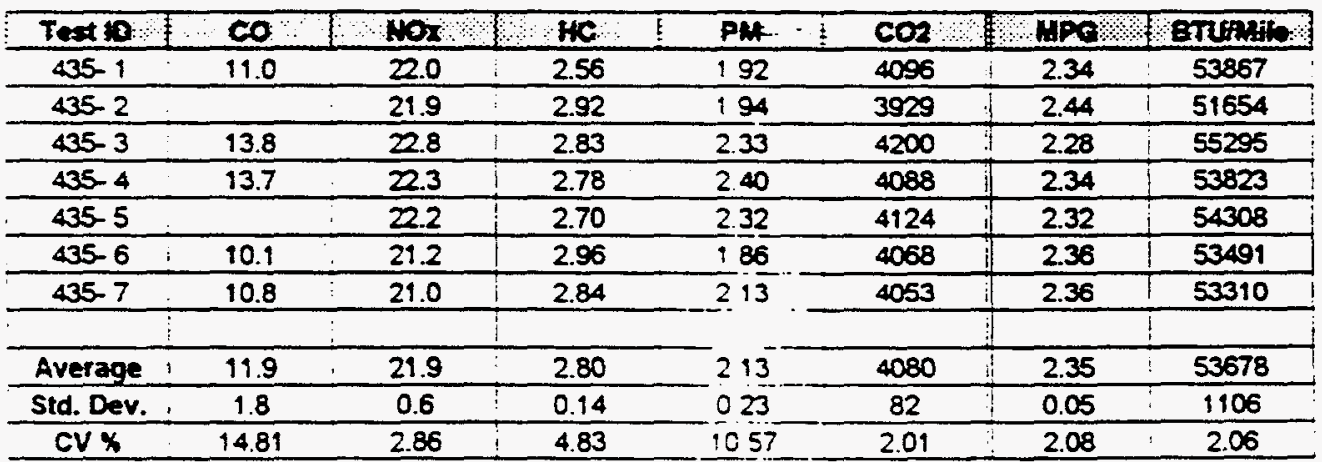


Test Sequence Number: 248

\section{WVU Test Reference Number HC-3220-D2}

Fieet Owner full Narne

Fieet Address

Fleet Address (City, State, Zip Code)

Vehicle Type

Vehicle ID Number (VIN)

Vehicle Manufecturer

Vehicle Model Year

Gross Vehicie Weight (GWM) (b)

Vehicie Total Curb Weight (lb)

Vehicde Simulated Weight (16)

Odonneter Reeding (mile)

Vehicle Transmiscion Type

Vehiche Tranemission Configuration

Number of Ades

Engine Type

Engive ID Number

Engine Displacement (liter)

Number Of Cylinders

Engine Rated Power (np)

Primary Fuel

Primary Fuel Sample ID

Secondary Fuel

Secondary Fuel Sample 10

Particulate Trap Manufacturer

Catalytic Converter Manufacturer

Test Cycie

Test Date
Hennepin County

320 Washington Ave.

Hopkins, MN 55343

Snow Plow
$2 H T T G D V T 1 P C 009272 \quad 322^{3}$

NVSTR

1993

55020

27510

27721

12409

Manual

Fuller RTX-11708LL 10-Spd

3

DDC 6V.92TA DDEC ॥

O6VF199374

9.05

6

$2 \pi$

D2

N-WV-1148

Not Applicable

Not Applicable

Not Applicable

Not Applicable

WVU-Trk

3/18/94
Emissions Results ( $\mathrm{g} / \mathrm{mile}$ )

Fuel Economy

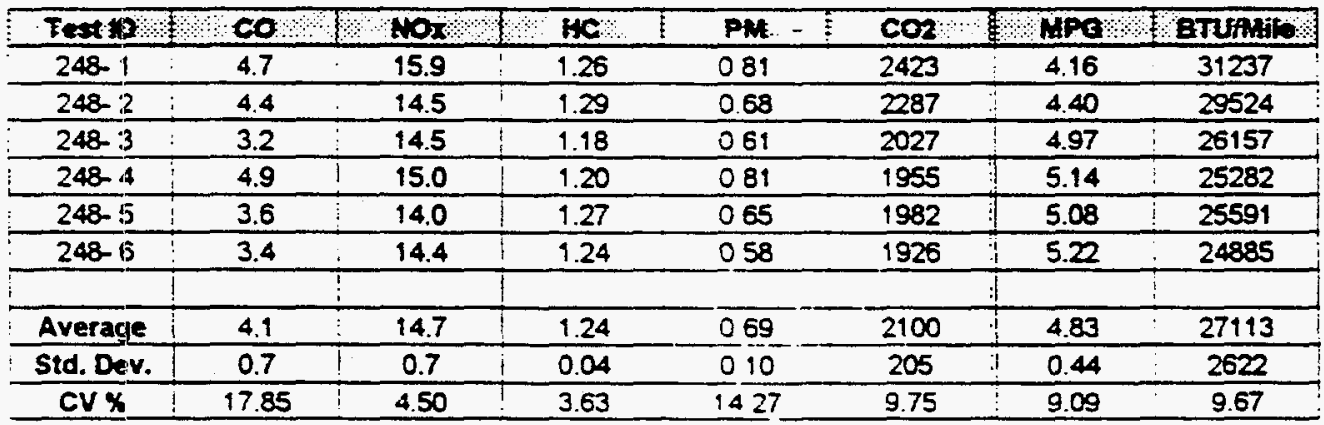


Test Sequence Number: 258

WVU Test Reference Number HC-3220-JA-TRK

Fieet Owmer Full Name

Fleot Address

Flect Address (City, State, Zip Code)

Vehicie Type

Vehicie ID Number (MN)

Vehicle Manufacturer

Venicle Model Year

Gross Venicte Weight (GWW) (Ib)

Vehicts Total Curt Weight (Ib)

Vehicie Simulated Weight (lb)

Odometer Reading (mile)

Vehicle Transmission Type

Vehicle Transmission Configuration

Number Of Axes

Engine Type

Engine 10 Number

Engine Displacement (liter)

Number Of Cytinders

Engine Rated Power (hp)

Primary Fuet

Primary Fuet Sample 10

Secondary Fuet

Secondary Fued Sample 10

Particulate Trap Manufacturer

Catalytic Converter Manufacturer

Test Cycle

Test Date

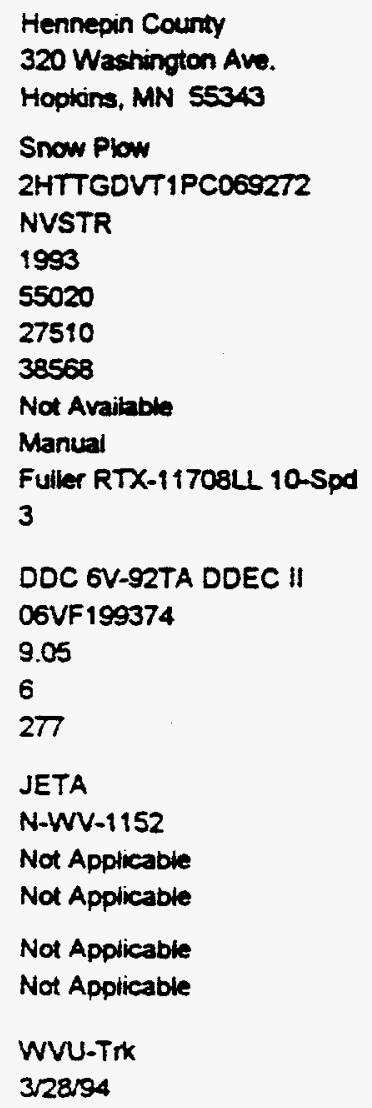

Fuel Economy

\begin{tabular}{|c|c|c|c|c|c|c|c|}
\hline Fest 10 & 60 & $\mathrm{NO}_{2}$ & H 10 & PA - I & $\cos$ & MPQ & Baunato \\
\hline $258-1$ & 4.3 & 13.1 & 1.40 & 085 & 2451 & 3.90 & 32334 \\
\hline $258-2$ & 4.1 & 13.7 & 1.40 & 084 & 2397 & 3.98 & 31631 \\
\hline $258-3$ & 4.8 & 13.1 & 1.25 & 083 & 2279 & 4.19 & 30041 \\
\hline $258-4$ & 3.9 & 12.6 & 1.31 & 0.78 & 2317 & 4.13 & 30533 \\
\hline & & & 1 & & & & \\
\hline Average & 4.3 & 13.1 & 1.34 & 082 & 2361 & 4.05 & 31135 \\
\hline Std. Dev. & 0.4 & 0.4 & 0.07 & 003 & 77 & 0.13 & 1040 \\
\hline CV $\%$ & 9.33 & 3.26 & 5.51 & 402 & 3.28 & 3.33 & 3.34 \\
\hline
\end{tabular}




\section{Test Sequence Number: 436}

WVU Test Reference Number

Flest Owner Fuil Name

Flent Addreas:

Flest Address (Clty, State, Zip Code)

Vehicle Type

Vehict ID Number (VIN)

Vehicle Manufacturer

Vehicle Model Year

Gross Venicie Weight (GWM) (Ib)

Vehicle Totol Curb Weight (Ib)

Vehicie Simutated Weight (Ib)

Odometer Reading (mile)

Vehicle Transmission Type

Vehicle Transmisaion Configuration

Number of Axtes

Engine Type

Engine ID Number

Engine Displacement (iter)

Number of Cylinders

Engine Rated Power (hp)

Primary Fuel

Primary Fuel Sample ID

Secondary Fue

Secondary Fuel Sample ID

Particulate Trap Manufacturer

Catantic Converter Manufacturer

Test Cycle

Test Date

\section{HC-3220-D1-5PK}

Hennepin County

320 Wrahington Ave.

Hoplains, MN 55343

Snow Plow

2HTTGDVT1PCOS9272

NVSTR

1993

55020

27510

38575

43751

Manual

Fuller RTX-11708LL 10-Spd

3

DDC GV-92TA DDEC II

O6VF199374

9.05

6

$2 \pi 7$

D1

95-04

Not Applicable

Not Applicable

Not Applicable

Not Applicable

WVU-Trk

5/9/95
Emissions Results (g/mile)

Fuel Economy

\begin{tabular}{|c|c|c|c|c|c|c|c|c|c|c|}
\hline Test 10 & 整 & $\mathrm{cos}$ & Nox & He & $!$ & Patin- & 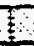 & $\operatorname{cog}$ & MPQ & STETHAit \\
\hline $436-1$ & $i$ & 5.2 & 13.9 & 1.43 & & 0.90 & & 2529 & 3.79 & 33219 \\
\hline $436-2$ & & 4.4 & 13.5 & 1.55 & & 0.78 & & 2525 & 3.80 & 33158 \\
\hline $436-3$ & $i$ & 4.5 & 13.2 & 1.50 & & 0.85 & & 2529 & 3.79 & 33204 \\
\hline $436-4$ & $!$ & 4.3 & 13.2 & 1.52 & 4 & 0.86 & $i$ & 2504 & 3.83 & 32870 \\
\hline $436-5$ & i & 4.6 & 12.6 & 1.50 & & 0.86 & $!$ & 2475 & 3.88 & 32506 \\
\hline & $!$ & & & & 1 & & 1 & & 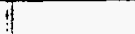 & 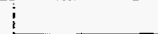 \\
\hline Average & 1 & 4.6 & 13.3 & 1.50 & & 0.85 & & 2512 & 3.82 & 32992 \\
\hline Std. Dev. & 1 & 0.4 & 0.5 & 0.04 & $!$ & 0.04 & $:$ & 23 & 0.04 & 306 \\
\hline CV $x_{1}$ & 1 & 7.67 & 3.58 & 2.96 & & 5.08 & & 0.93 & 0.93 & 0.93 \\
\hline
\end{tabular}


Test Sequence Number: 453

WVU Test Reference Number BC-3220-D1-5PK-R

Fleet Owner Full Name

Fleet Address

Fleet Addreas (City, State. Zip Code)

Venicte Type

Vehicte 10 Number (VIN)

Vehicle Marufactures

Vehicle Model Year

Gross Vehicie Weight (GWW) (1b)

Vehicie Total Curb Weight (b)

Vehicis Simulated Weight (b)

Odonneter Reading (mile)

Vehicle Transmission Type

Vehicle Tranamission Configuretion

Number Of Axies

Engine Type

Engine ID Number

Engine Displacement (lites)

Number Of Cylinders

Engine Rated Power (hp)

Primany Fuet

Primary Fuet Sample ID

Secondary Fuet

Secondary Fuet Sample ID

Particulate Trap Manutacturer

Catalytic Converter Manufacturer

Test Cycie

Test Date
Hennepin County

320 Washington Ave.

Hopkins, MN 55343

Snow Plow

2HTTGDVTIPCOS9272

NVSTR

1993

55020

27510

34409

44349

Manual

Fuller RTX-11708LL 10-Spd

3

DDC SV-92TA DDEC II

O6VF199374

9.05

6

277

D1

95-04

Not Applicabie

Not Applicable

Not Appticable

Not Applicable

WVU-Trk

s/20ros

Emissions Results (g/mile)

Fuel Economy

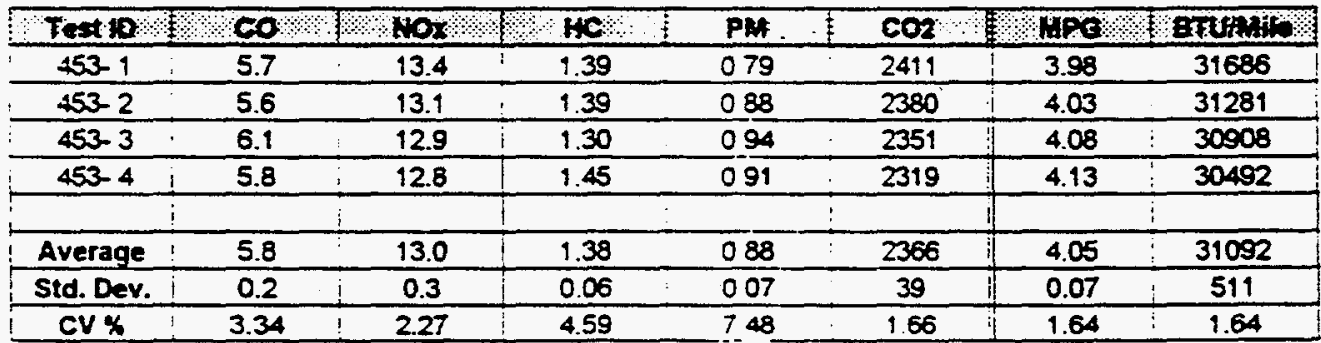


Test Sequence Number: 262

\section{WVU Test Reference Number HC-3221-E100}

Fieel Owner Full Name

Fleed Address

Fleel Address (City. State. Zip Code)

Vehick Type

Vehicie ID Number (VIN)

Vehich Manufacturer

Vehicke Model Year

Groes Vehicis Weight (GWM) (Ib)

Vehicle Total Curb Weight (lb)

Vohicle Simubted Weight (lb)

Odorneter Reading (mile)

Vehicte Transmission Type

Vehicte Transmission Configuration

Number of Axtes

Engine Type

Engirle ID Number

Engirve Displacement (liter)

Number Of Cyinders

Engire Rated Power (hp)

Primary Fued

Primary Fued Sample 10

Secondary Fuel

Secondary Fued Sample ID

Particulate Trap Manufacturer

Catahtic Converter Manufacturer

Test Cycle

Test Date

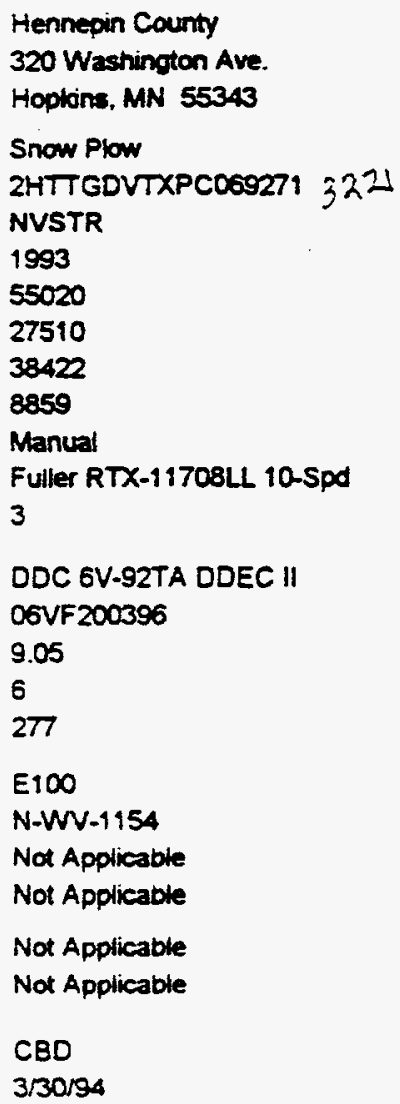

Emissions Results ( $\mathrm{g} / \mathrm{mile}$ )

Fuel Economy

\begin{tabular}{|c|c|c|c|c|c|c|c|c|}
\hline Fest $x$ & $c 0$ & $10 x$ & HC & PM $\ldots$ & 602 & 2 & Mas & Brutenit \\
\hline $262-1$ & 82.6 & 13.1 & 11.90 & 105 & 4427 & & 1.24 & 61231 \\
\hline $262-2$ & 87.3 & 13.1 & 12.29 & 110 & 4367 & $\because$ & 1.25 & 60823 \\
\hline $252-3$ & 86.6 & 13.9 & 12.52 & 113 & 4462 & $i$ & 1.23 & 61849 \\
\hline & $i$ & & & & & 1 & & 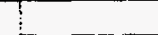 \\
\hline Average & 85.5 & 13.4 & 12.24 & 109 & 4419 & & 1.24 & 61301 \\
\hline Std. Dev. & 2.5 & 0.5 & 0.31 & 0.04 & 48 & 1 & 0.01 & 516 \\
\hline CV $\%$ & 2.95 & 3.59 & 2.54 & 353 & 1.08 & $\pi$ & 0.84 & 0.84 \\
\hline
\end{tabular}




\section{Test Sequence Number: 432}

\section{WVU Test Reference Number HC-3221-E100-TCBD}

Flect Owner Ful Name

Fleot Addrese

Fleet Address (Cidy, State, Zip Code)

Venicle Trpe

Vehicie ID Number (VIN)

Vehicie Manufacturer

Vehicio Model Year

Gross Venicio Weight (GWW) (b)

Vehiche Tobal Curb Weight (Ib)

Vehicke Simulated Weight (b)

Odometer Reading (mile)

Vehicte Transmission Type

Vehicie Transmission Configuration

Number of Ades

Engine Type

Engine ID Number

Engine Displacement (liter)

Number of Cylinders

Engine Rated Power (hp)

Primary Fued

Primary Fuel Sample ID

Secondary Fuel

Secondary Fuel Sample ID

Particulate Trap Manufacturer Catalytic Converter Manufacturer

Test Cycie

Test Date
Hennepin County

320 Warhington Ave.

Hopkine, MN 55343

Snow Plow
2 HTTGOVTXPCO69271 327
NVSTR
1993
55020
27510
38575
30373
Manual
Fuller RTX-11708LL 10-Spd
3
DDC 6V-92TA DOEC II
06 VF200396
9.05
6
277
E100
$95-03$
Not Applicable
Not Applicable
Not Applicable
N/A
Truck-CBD
$5 / 5 / 95$
Emissions Results ( $\mathrm{g} / \mathrm{mile})$

Fuel Economy

\begin{tabular}{|c|c|c|c|c|c|c|c|c|c|c|}
\hline Fet10 & 1 & $\infty$ & & $\operatorname{mog}$ & He & PM & $\cos$ & H & MPe: & ETunato \\
\hline $432-1$ & 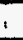 & & & 14.3 & 6.49 & 0.58 & 3687 & & 1.53 & 49596 \\
\hline 432.2 & $:$ & 10.2 & & 13.9 & 6.02 & 066 & 3797 & $-i$ & 1.49 & 50990 \\
\hline $432-3$ & & 9.1 & & 14.0 & 5.50 & 067 & 3804 & 4 & 1.49 & 51040 \\
\hline $432-4$ & $i$ & 10.9 & & 13.8 & 5.30 & 0.73 & 3796 & $I$ & 1.49 & 50964 \\
\hline $432-5$ & $\vdots$ & 10.3 & $!$ & 13.8 & & 073 & 3796 & $i$ & 1.50 & 50809 \\
\hline 432.6 & $\vdots$ & 8.6 & & 14.1 & 7.74 & 0.78 & 3699 & & 1.53 & 49687 \\
\hline & $!$ & & & & 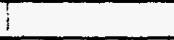 & & & & & $T$ \\
\hline Average & 1 & 9.8 & & 14.0 & 6.21 & 069 & 3753 & & 1.50 & 50514 \\
\hline Std. Dev. & 1 & 1.0 & $i$ & 0.2 & 0.97 & 007 & 55 & it & 0.02 & 681 \\
\hline CV $\%$ & $!$ & 9.83 & 1 & 1.54 & 15.68 & 1039 & 1.45 & 1 & 1.36 & 1.35 \\
\hline Teat 10 & 8 & eateon & 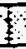 & Heto & Carscio & Ratc & Ondtc & & & \\
\hline $432-1$ & $i$ & 0.81 & & 0.18 & $\vdots$ & & & & & \\
\hline $432-2$ & 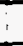 & 0.79 & & 0.15 & 0.91 & & 4.45 & & & \\
\hline $432 \cdot 3$ & & 0.69 & & 0.14 & 0.92 & & 4.09 & & & \\
\hline $432-4$ & : & 0.61 & & 0.15 & 0.82 & & 3.90 & & & \\
\hline $432-5$ & $\rightarrow$ & 0.65 & & 0.14 & 0.80 & & & & & \\
\hline 432.6 & 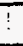 & 0.57 & & 0.14 & 0.78 & & 5.37 & & & \\
\hline Average & 1 & 0.70 & & 0.15 & 0.85 & & 445 & & & \\
\hline Std. Dev. & & 0.08 & & 0.01 & 0.07 & & 0.65 & & & \\
\hline CVx & & 11.40 & & 10.07 & 7.74 & & 1467 & & & \\
\hline
\end{tabular}




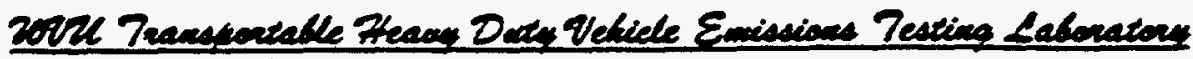

Department of Mechanical and aesospace Engineering, West Virginia University, Morgantown, WV 26506 Tel: (304) 293-3111 FAX: (304) 293-8832

Test Sequence Number: 250

WVU Test Reference Number HC-3221-E100

Fieet Owner Full Name

Fiect Addres:

Fled Addreses (City, State, Zip Code)

Vehicie Typo

Vehicle ID Number (VIN)

Vehicle Manufacturer

Vehicle Model Year

Groes Vehich Weight (GWM) (b)

Vehicte Total Curb Weight (lb)

Vehick Simutated Weight (Ib)

Odomster Reading (mile)

Vehiche Tranamiasion Type

Vehich Transmission Configuration

Number Of Axtes

Enginat Type

Engine 1D Number

Engine Displacement (iter)

Number of Cylinders

Engine Rated Power (hp)

Primary Fuet

Primary Fuel Sample ID

Secondary Fuel

Secondary Fuel Sample ID

Particulate Trap Manufacturer

Catalytic Comverter Manufacturer

Test Cycle

Test Date
Hennepin County

320 Washington Ave.

Hopkins, MN 55343

Snow Plow

2HTTGDVTXPCOS9271

NVSTR

1993

55020

27510

27672

8842

Manual

Fuller RTX-11708LL 10-Sp

3

DDC GV-92TA DDEC II

06VF200396

9.05

6

277

E100

$N-W-1154$

Not Applicable

Not Applicable

Not Applicable

Not Applicable

WVU-Trk

3/19/94

Emissions Results (g/mile)

Fuel Economy

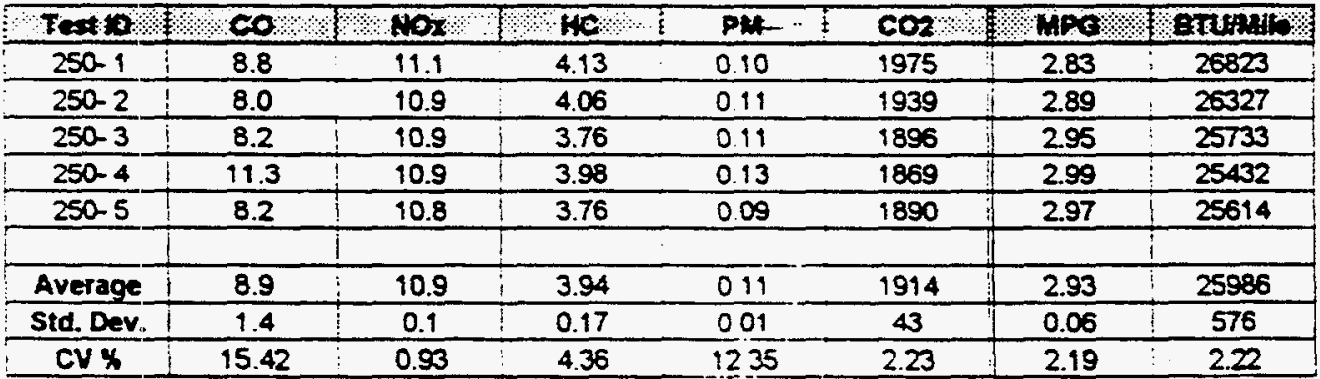


Test Sequence Number: 261

WVU Test Reference Number HC-3221-E100-TRK

Fleet Owner Full Name

Fleet.Address

Fleet Address (City, State. Zip Code)

Vehicle Type

Vehicle tD Number (VIN)

Vehiclo Manufacturer

Vehicle Model Year

Gross Venicle Weight (GM) (Ib)

Vehicle Total Curb Weight (ID)

Vehicie Simulated Weight (lb)

Odometer Reading (mile)

Vehicle Transmiscion Type

Vehicle Transmission Configuration

Number Of Axes

Engine Type

Engine ID Number

Engine Displacement (liter)

Number Of Cylinders

Engine Rated Power (hp)

Primary Fuel

Primary Fued Sample ID

Secondary Fuei

Secondary Fuet Sampie 10

Particulate Trap Manufacturer

Catalytic Conventer Manufacturer

Test Cycle

Test Date
Hennepin County

320 Washington Ave.

Hopkins, MN 55343

Snow Plow

2HTTGDVTXPC069271

NVSTR

1993

55020

27510

38422

Not Available

Manual

Fuller RTX-11708LL 10-Spd

3

DDC GV-92TA DDEC II

Q6VF200396

9.05

6

$2 \pi 7$

E100

N-WV-1154

Not Applicable

Not Applicable

Not Applicable

Not Applicable

WVU-TrK

3/30/94
Emissions Results (g/mile)

Fuel Economy

\begin{tabular}{|c|c|c|c|c|c|c|c|c|}
\hline Fest 10 & $3 \quad c 0$ & $\sin$ & 3 & 140 & $P$ P & $\mathrm{CO}$ & MPES & fezernits \\
\hline $251-1$ & 8.3 & 12.2 & & 4.97 & 0.32 & 2493 & 2.26 & 33664 \\
\hline $261-2$ & 6.2 & 12.5 & $\vdots$ & 5.10 & 0.30 & 2432 & 2.32 & 32805 \\
\hline $261-3$ & 6.7 & 12.3 & $!$ & 4.87 & 0.29 & 2405 & 2.34 & 32416 \\
\hline $251-4$ & i & 12.4 & & 4.97 & 0.29 & 2347 & 2.40 & 31616 \\
\hline $261-5$ & 8.1 & 12.1 & & 4.93 & 0.28 & 2367 & 2.38 & 31949 \\
\hline & 1 & & & & & & 7 & $i$ \\
\hline Average & 7.3 & 12.3 & & 4.97 & 0.30 & 2409 & 2.34 & 32490 \\
\hline Std. Dev. & 1.0 & 0.2 & & 0.08 & 0.02 & 58 & 0.06 & 797 \\
\hline CV $x$ & 13.76 & 1.30 & & 1.70 & 5.45 & 2.39 & 2.43 & 2.45 \\
\hline
\end{tabular}

\begin{tabular}{|c|c|c|c|c|c|}
\hline Test 10 & C2HSoH & FCFO & CHFCHO & RitC & OMHC \\
\hline $261-1$ & 2.48 & 0.11 & 0.50 & 4.31 \\
\hline 261.2 & 2.54 & 0.12 & 0.61 & 4.49 \\
\hline 261.3 & 2.42 & 0.10 & 0.52 & 4.25 \\
\hline 261.4 & 2.45 & 0.19 & 0.60 & 4.36 \\
\hline 261.5 & 2.12 & 0.12 & 0.59 & 4.21 \\
\hline & & & & \\
\hline Average & 2.40 & 0.11 & 0.56 & 4.32 \\
\hline Std. Dev. & 0.16 & 0.01 & 0.05 & 0.11 \\
\hline CV\% & 5.87 & 7.43 & 8.72 & 2.52 \\
\hline
\end{tabular}


Test Sequence Number: 431

\section{WVU Test Reference Number HC-3221-E100-5PK}

Fleet Oumer Full Name

Fleet Addreas

Fleet Addrese (City. State, Zip Code)

Vehicle Type

Vehicle ID Number (VIN)

Vehicie Manufactures

Vehicle Model Year

Gross Vehich Weight (GWW) (lb)

Vehiche Total Curb Weight (Ib)

Vehiche Simutated Weight (Ib)

Odometer Reading (mile)

Vehiche Tranemisaion Type

Vehichs Tranemiseion Configuration

Number Of Ades.

Engine Type

Engind ID Number

Engina Displacement (liter)

Number of Cylinders

Engine Rated Power (hp)

Primary Fuel

Primary Fuel Sample ID

Secondary Fuet

Secondary Fuel Sample 10

Particulate Trap Manufacture

Catahyic Converter Manufacturer

Test Cycle

Test Date
Hennepin County

320 Washington Ave.

Hopkins, MN 55343

Snow Plow

2HTTGOVTXPC06927:

NVSTR

1993

55020

27510

38575

30353

Manual

Fuller RTX-11708LL 10-Spd

3

DDC 6V-92TA DDEC II

D6VF200396

9.05

6

277

E100

9503

Not Applicable

Not Applicabie

Not Applicable

N/A

WVU-Tik

5/5/95
Emissions Results (g/mile)

Fuel Economy

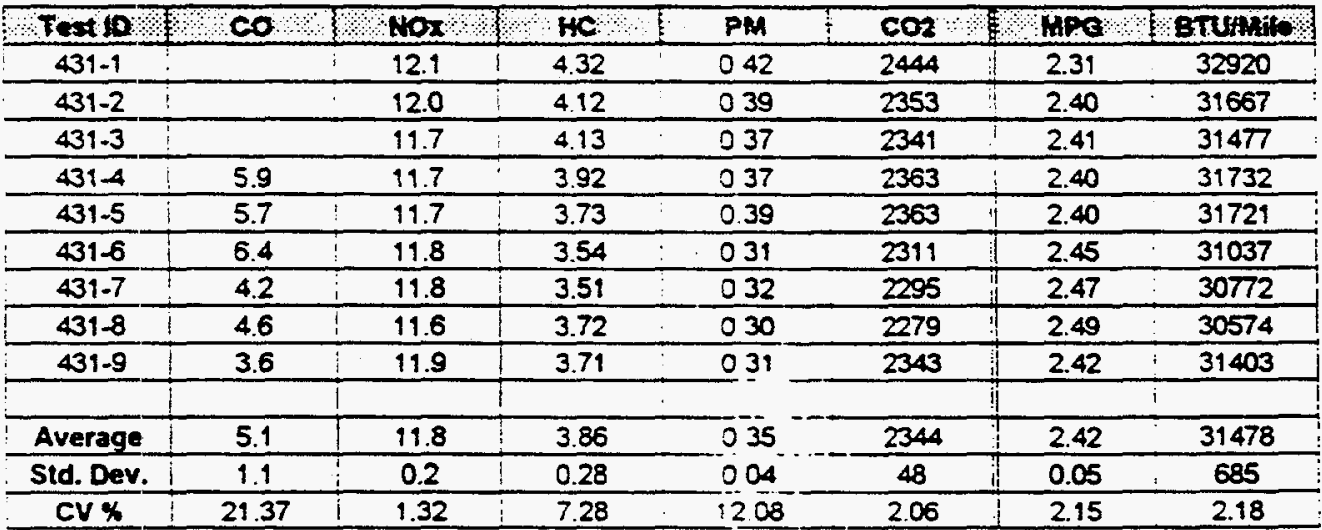

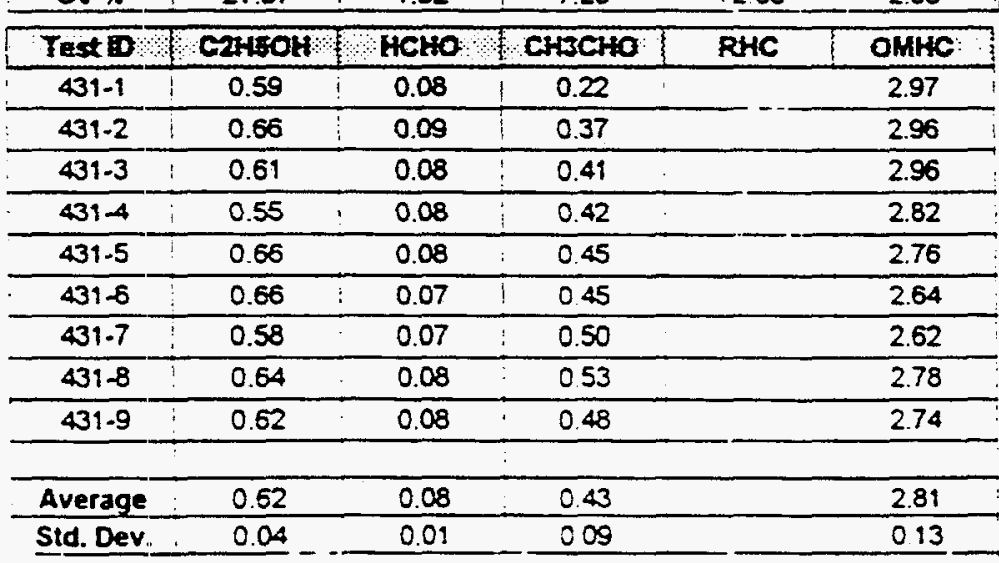


Test Sequence Number: 454

WVU Test Reference Number HC-3221-E100-5PK-R

Fieet Ommer Full Name

Fleat Addres:

Flect Address (City, State, Zip Code)

Vehicle Type

Vehicle ID Number (VIN)

Vehich Manutacturer

Vehicle Model Year

Groes Vehich Weight (GWW) (b)

Vehich Total Curb Weight (Ib)

Vehicis Simutated Weight (lb)

Odometer Reading (mila)

Vehicle Tranemission Typo

Vehicle Transmission Configuration

Number Of Axkes

Engine Type

Engine 10 Number

Engine Displacement (liter)

Number of Cylinders

Engine Rated Power (hp)

Primary Fuel

Primary Fued Sample ID

Secondary Fuel

Secondary Fuel Sample ID

Panticulate Trap Manufacturer

Catahyic Converter Manufacturer

Test Cycle

Test Date
Henmepin Coumy

320 Wartington Av.

Hopions, MN 55343

Snow Plaw

2HTTGDVTXPCOOS271

NVSTR

1993

55020

27510

38575

31054

Manual

Fuller RTX-11708LL 10-Spd

3

DDC 6V-92TA DDEC II

OSVF20039S

9.05

6

277

$E 100$

$95-03$

Not Applicable

Not Applicable

Not Applicable

Not Applicabie

WU-Trk

5/21/95
Emissions Results (g/mile)

Fuel Economy

\begin{tabular}{|c|c|c|c|c|c|c|c|c|c|c|}
\hline$T=x+3$ & 8 & $\mathrm{cos}$ & nose & H HC & PA & CO2: & 8 & Has & 3 & entrang \\
\hline 4541 & $!$ & 8.9 & 11.6 & 4.30 & 0.44 & 2596 & $: i$ & 2.18 & + & 34908 \\
\hline $454-2$ & $\vdots$ & 7.9 & 11.4 & 4.00 & 0.37 & 2530 & $\mathrm{i}$ & 2.24 & $\vdots$ & 33999 \\
\hline $454-3$ & $\vdots$ & 8.4 & 11.2 & 4.12 & 035 & 2500 & i & 2.26 & & 33613 \\
\hline 454.4 & $i$ & 6.0 & 11.2 & 3.85 & 042 & 2471 & 4 & 2.29 & 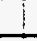 & 33171 \\
\hline 4545 & $\vdots$ & 7.3 & 11.0 & 3.81 & 036 & 2445 & & 2.31 & 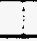 & 32847 \\
\hline & 1 & & & 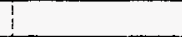 & & & $!$ & & 1 & \\
\hline Average & $i$ & 7.7 & 11.3 & 4.02 & 039 & 2508 & $\because$ & 2.26 & 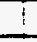 & 33707 \\
\hline Std. Dev. & 1 & 1.1 & 0.2 & 0.20 & 004 & 58 & 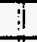 & 0.05 & 1 & 801 \\
\hline CV $\%$ & $T$ & 14.29 & 2.01 & 5.04 & 1001 & 2.33 & 7 & 2.35 & $i$ & 2.38 \\
\hline Tet D & 3 & ores:ons & HCHO & CXNANO & $R+x$ & Orarte. & & & & \\
\hline 4541 & 1 & 0.78 & 0.09 & 0.45 & & 3.15 & & & & \\
\hline 4542 & $i$ & 0.82 & 0.09 & 0.51 & & 3.01 & & & & \\
\hline $454-3$ & $!$ & 0.81 & 0.08 & 0.47 & & 307 & & & & \\
\hline $454-4$ & $\vdots$ & 0.82 & 0.09 & 0.56 & & 2.94 & & & & \\
\hline 4545 & $\vdots$ & 0.74 & 0.08 & 0.54 & & 2.88 & & & & \\
\hline & $\vdots$ & & & : & & & & & & \\
\hline Average & $!$ & 0.80 & 0.08 & 0.51 & & 3.01 & & & & \\
\hline Std. Dev. & 1 & 0.03 & 0.00 & 0.05 & & 0.11 & & & & \\
\hline CVX & & 4.25 & 3.08 & 9.15 & & 3.53 & & & & \\
\hline
\end{tabular}


Test Sequence Number: 260

WVU Test Reference Number

Fleet Owner Fult Name

Finet Addreas:

Fleot Address (City, State, Zip Code)

Vehicle Type

Vehicle ID Number (MN)

Vehicle Manufacturer

Vehice Moclet Year

Gros Vehicle Weigh (GWM) (Ib)

Vehicle Total Curb Weight (lb)

Vehicle Simulated Weight (lb)

Odometer Reeding (mile)

Vehicle Transmission Type

Vehicle Transmission Configuration

Number Of Ades

Engim: Type

Engine ID Number

Engina Displacement (liter)

Number Of Cyinders

Engine Rated Power (hp)

Primary Fuet

Primary Fuel Sample 10

Secondary Fuel

Secondary Fuel Sample to

Particulate Trap Manufacturer

Catahyic Converter Manufacturer

Test Cycie

Test Date

\section{HC-3228-E100}

Hennepin County

320 Waehington Ave.

Hookins. MN 55343

Snow Plow

2HTTGDVTBPCOES270

NVSTR

1993

55020

27510

38422

5367

Manual

Fuller RTX-11708LL 9-Spd

3

DDC GV-92TA DDEC II

O6VF 200397

9.05

6

277

E100

N-W-1154

Not Applicable

No Applicable

Not Applicable

Not Applicable

CBD

$3 / 29 / 94$
Emissions Results (g/mile)

Fuel Economy

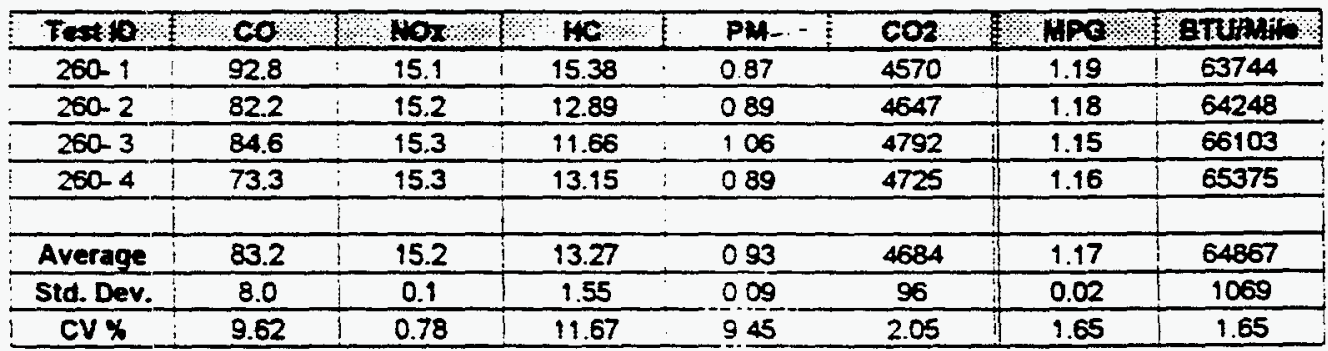




\section{Test Sequence Number: 251}

WVU Test Reference Number

\author{
Fiect Owner Full Name \\ Fieet Addreas \\ Flect Address (City. State, Zip Code) \\ Vehich Type \\ Vehict 10 Number (VIN) \\ Vehicto Manutacturer \\ Vehicie Model Year \\ Gross Vehicie Weight (GWM) (b) \\ Vehiche Total Curb Weight (Ib) \\ Vehicte Simulated Weight (b) \\ Odorneter Reading (mile) \\ Vehicie Transmission Type \\ Vehicie Tranamision Configuration \\ Number Of Ades
}

Engine Type

Engine ID Number

Engine Displacement (itter)

Number Of Cylinders

Engine Rated Power (hp)

Primary Fued

Primary Fued Sample iD

Secondary Fuel

Secondary Fuel Sample 10

Particulate Trap Manufacturer

Catatytic Converter Manufacturer

Test Cycle

Test Date

\section{HC-3228-E100}

Hennepin County

320 Washington Ave.

Hopkins, MN 55343

Snow Plow

2HTTGDVT8PC069270

NVSTR

1993

55020

27510

27672

5175

Manual

Fuller RTX-11708LL 9Spd

3

DDC 6V-92TA DDEC \|

OSVF200397

9.05

6

$2 \pi$

E100

N-WV-1154

Not Applicable

Not Applicable

Not Applicable

ACDELCO

WVU-Trk

$321 / 94$
Emissions Results ( $g / m i l e)$

Fuel Economy

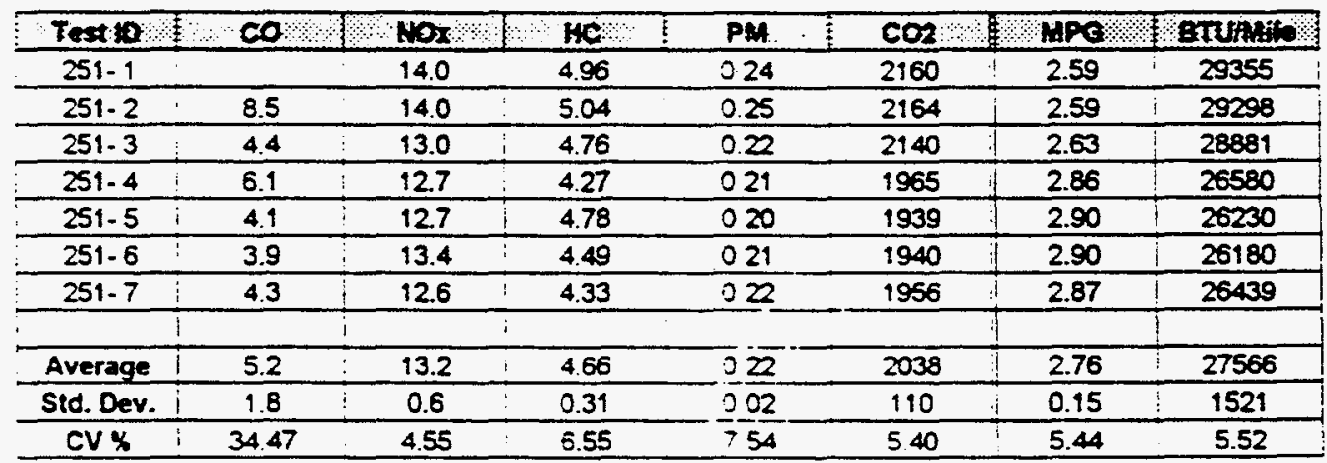




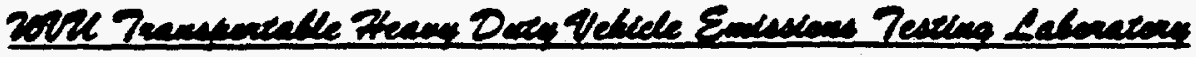

Department of Mecharical and aerospace Engineering, Weat Virginia University, Morgantown, WV 26506

Tel: (304) 293-3111 FAX: (304) 293-3832

Test Sequence Number: 433

WVU Test Reference Number

Fleet Owner Full Name

Fleet Addres:

Fleet Address (City, State, Zip Code)

Venicte Type

Vehice ID Number (VIN)

Vehicie Manutacturer

Vehici Model Year

Groesl Vehicle Weight (GWW) (b)

Venicte Total Curt Weight (Ib)

Venicte Simulated Weight (b)

Odorneter Reading (mile)

Vehicie Tranamisaion Type

Vehicie Tranamission Configuration

Number Of Ades

Engine Type

Engine ID Number

Engine Dispbeement (liter)

Number Of Cylinders

Engina Rated Power (hp)

Primary Fued

Primary Fuet Sample 10

Secondary Fuet

Secondary Fuet Sample ID

Particulate Trap Manufacturer

Catalytic Converter Manufacturer

Test Cycle

Test Date

\section{EC-3228-E100-TCBD}

Hernepin County

320 Wearington Aw.

Hoplins, MN 55343

Snow Plow

2HTTGOVT8PCOS9270

NVSTR

1993

55020

27510

38575

17170

Manual

Fulier RTX-11708LL 9-Spd

3

DDC GV-92TA DDEC II

O6VF200397

9.05

6

277

E100

95-03

Not Applicable

Not Applicable

Not Applicable

N/A

Truck-CBD

5/6/95
Emissions Results (g/mile)

Fuel Economy

\begin{tabular}{|c|c|c|c|c|c|c|c|c|}
\hline Fent 2 & 80 & nos: & te & 1 & PRAP & $\mathrm{cos}$ & SPPS & 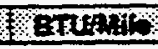 \\
\hline $433-1$ & 122.7 & 15.2 & 17.58 & & 1.35 & 4112 & 1.31 & 57940 \\
\hline $433-2$ & 121.5 & 15.0 & 16.94 & 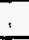 & 1.44 & 4119 & 1.31 & 57975 \\
\hline $433-3$ & 113.5 & 14.6 & 15.38 & $\vdots$ & 1.56 & 4156 & 1.30 & 58254 \\
\hline $433-4$ & 111.7 & 14.6 & 16.57 & & 1.42 & 4115 & 1.32 & 57713 \\
\hline $433-5$ & 111.5 & 14.8 & 16.12 & & 1.38 & 4168 & 1.30 & 58401 \\
\hline & & & & & & & & \\
\hline Average & 116.2 & 14.8 & 16.52 & 1 & 1.43 & 4134 & 1.31 & 58057 \\
\hline std. Day. & 5.5 & 0.3 & 0.83 & + & 0.08 & 26 & 0.01 & 272 \\
\hline CV $x$ & 4.70 & 1.84 & 5.02 & & 5.77 & 0.63 & 0.47 & 0.47 \\
\hline
\end{tabular}

\begin{tabular}{|c|c|c|c|c|c|c|}
\hline Tetis & cturos & Vhenes & Cracko & Fate: & & Olakte \\
\hline $433-1$ & 6.53 & 0.88 & 3.88 & & & 15.38 \\
\hline $433-2$ & 5.51 & 0.90 & 3.48 & & 1 & 14.42 \\
\hline $433-3$ & 5.50 & 0.84 & 3.31 & & $i$ & 13.37 \\
\hline $433-4$ & 5.50 & 0.89 & 3.44 & & 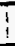 & 14.17 \\
\hline $433-5$ & 5.51 & 0.86 & 3.38 & & : & 13.86 \\
\hline & 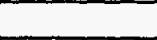 & 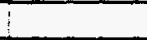 & i & & 1 & \\
\hline Average & 5.71 & 0.88 & 3.50 & & & 14.24 \\
\hline Std. Dev. & 0.46 & 0.02 & 0.23 & & 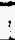 & 0.75 \\
\hline CVx & 8.01 & 285 & 6.44 & & i & 5.26 \\
\hline
\end{tabular}




\section{Test Sequence Number: 434} WVU Test Reference Number

Fieet Owner Full Name

Fleet Addreas

Fleet Address (City, State, Zip Code)

Vehicle Type

Vehicle ID Number (VN)

Vehicie Menutacturer

Vehicie Model Year

Grose Vehicte Weight (GWW) (b)

Venicte Total Curb Weight (Ib)

Vehicle Simutated Weight (Ib)

Odometer Reading (mite)

Vehicte Transmission Type

Vehicie Transmission Configuration

Number Of Axies

Engine Type

Engine ID Number

Engine Displacement (itter)

Number Of Cylinders

Engine Rated Power (hp)

Primary Fued

Primary Fuel Sampie 10

Secondary fuet

Secondary Fuel Sample ID

Particulate Trap Manufacturer Catalytic Converter Manufacturer

Test Cycie

Test Date

\section{HC-3228-E100-5PK}

Hennepin County

320 Washington Ave.

Hopkins, MN 55343

Srow Plow

2HTTGDVT8PCOSO270

NVSTR

1993

55020

27510

38575

17200

Manual

Fullet RTX-11708LL 9-Spd

3

DDC 6V-92TA DDEC ॥

06VF200397

9.05

6

$2 \pi$

E100

95-03

Not Applicable

Not Applicable

Not Applicable

Not Applicable

WVU-Trk

5/6/95
Emissions Results (g/mile)

Fuel Economy

\begin{tabular}{|c|c|c|c|c|c|c|c|}
\hline Teat 10 & $c 0$ & $\mathrm{Nax}$ & twe & PA. & $\cos :$ & MPQ & entunis \\
\hline 4341 & 28.4 & 122 & 7.23 & 0.60 & 2474 & 2.25 & 33801 \\
\hline 4342 & 25.7 & 12.1 & 6.80 & 0.54 & 2422 & 2.30 & 33059 \\
\hline 4343 & 26.3 & 12.0 & 6.95 & 058 & 2457 & 2.27 & 33538 \\
\hline $434-4$ & 24.8 & 11.9 & 7.30 & 0.55 & 2482 & 2.25 & 33849 \\
\hline & 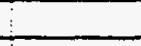 & 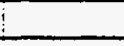 & & & & & \\
\hline Average & 26.2 & 12.0 & 7.07 & 056 & 2459 & 2.26 & 33562 \\
\hline Std. Dev. & 1.4 & 0.1 & 0.24 & 003 & 27 & 0.02 & 362 \\
\hline CV \% & 5.22 & 1.18 & 3.33 & 490 & 109 & 1.09 & 1.08 \\
\hline
\end{tabular}

\begin{tabular}{|c|c|c|c|c|c|}
\hline Test & eztions & Hero & Catscho & RHC & OHHC \\
\hline 4341 & 3.40 & 0.27 & 1.84 & & 6.68 \\
\hline 4342 & 4.27 & 0.26 & 1.85 & & 6.75 \\
\hline 4343 & 3.45 & 0.29 & 1.94 & & 6.57 \\
\hline $434-4$ & 3.86 & 0.28 & 1.88 & & 6.92 \\
\hline & & & & & \\
\hline Average & 3.74 & 0.28 & 1.88 & & 6.73 \\
\hline Std. Dev. & 0.41 & 0.01 & 0.04 & & 0.14 \\
\hline CV\% & 10.83 & 3.75 & 2.21 & & 2.14 \\
\hline
\end{tabular}


Test Sequence Number: 259

WVU Test Reference Number HC-3228-E100-TRK

\begin{tabular}{|c|c|}
\hline $\begin{array}{l}\text { Fleet Owner Full Name } \\
\text { Fiect Address } \\
\text { Fleet Address (City, State, Zip Code) }\end{array}$ & $\begin{array}{l}\text { Hennepin County } \\
320 \text { Washington Ave. } \\
\text { Hopians, MN } 55343\end{array}$ \\
\hline Vehicie Type & Snow Plow \\
\hline Venicio ID Number (VIN) & 2HTTGDVT8PCOS9270 \\
\hline Vehicie Manufacturer & NVSTR \\
\hline Vehicle Model Year & 1993 \\
\hline Gross Vehicle Weight (GWW) (b) & 55020 \\
\hline Vehicle Total Curo Weight (ib) & 27510 \\
\hline Vehiche Simulated Weight (lb) & 38422 \\
\hline Odometer Reading (mile) & 5349 \\
\hline Vehicle Transmission Type & Manual \\
\hline Vehich Transmission Configuration & Fuller RTX-11708LL 9Spd \\
\hline Number Of Axtes & 3 \\
\hline Engine Type & DDC 6V.92TA DDEC II \\
\hline Engine ID Number & 06VF200397 \\
\hline Engine Displacement (liter) & 9.05 \\
\hline Number of Cytinders & 6 \\
\hline Engine Rated Power (hp) & 277 \\
\hline Primary Fuel & E100 \\
\hline Primary Fuel Sample 10 & $N-W V-1154$ \\
\hline Seconctary Fuel & Not Applicable \\
\hline Seconclary Fuer Sample ID & Not Applicable \\
\hline Particulate Trap Manufacturer & Not Applicable \\
\hline Catarytic Converter Manufacturex & Not Applicable \\
\hline Test Cyrcie & WVU-Trk \\
\hline Test Date & $3 / 29 / 94$ \\
\hline
\end{tabular}

Emissions Results ( $g / m i l e)$

Fuel Economy

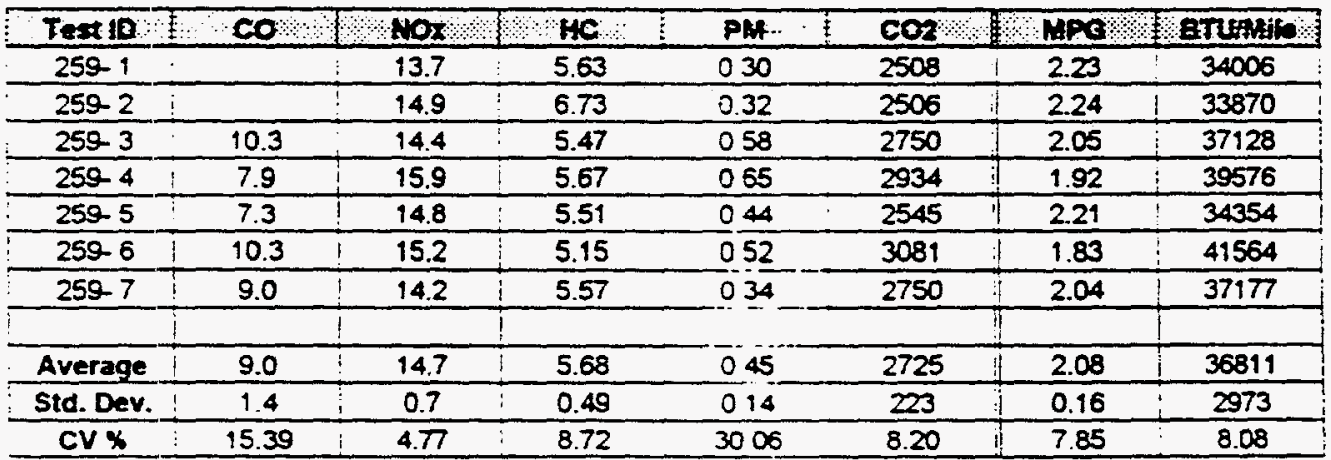


Test Sequence Number: 460

WVU Test Reference Number

Fieet Owner Full Name

Fleot Addreas

Fleet Address (City, State, Zip Code)

Vehicle Type

Vehicie ID Number (VN)

Vehicte Manutecturer

Vehicto Model Year

Grose Vehicis Weight (GWM) (lb)

Vehicte Total Curb Weight (b)

Vehicie Simutod Weight (lb)

Odometer Reading (mile)

Vehicie Transmiasion Type

Vehicle Transmiscion Configuration

Number Of Ades

HC-3228-E100-5PK-R

Engine Type

Engine ID Number

Engine Displacement (liter)

Number Of Cylinders

Engine Rated Power (hp)

Hensepin County

320 Washington Ave.

Hoakins, MN 55343

Snow Piow

2HTTGDVT8PCOS9270

NVSTR

1993

55020

27510

38575

17603

Manual

Fuller RTX-11708LL S-Spd

3

DDC 6V-92TA DDEC II

06VF200397

9.05

6

277

Primary Fuel

Primary Fuel Sample ID

Secondary Fued

Secondary Fuet Sample ID

Particulate Trap Manufacturer

Catantic Converter Manufacturer

E100

95-03

Not Applicable

Not Applicable

Not Applicable

Not Applicable

Test Cycle

WVU-Trk

Test Date

$5 / 23 / 95$

Emissions Results (g/mile)

Fuel Economy

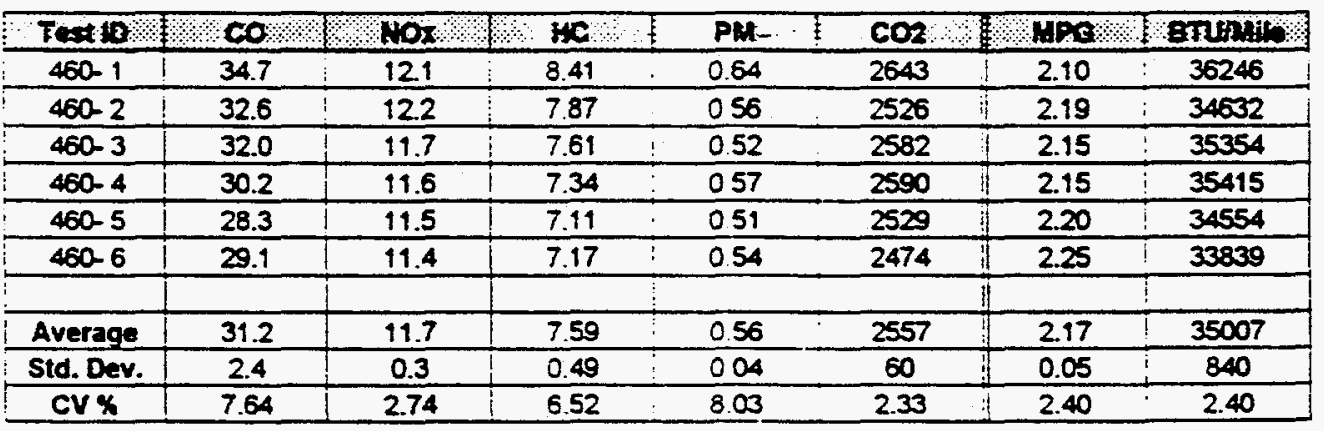

\begin{tabular}{|c|c|c|c|c|c|}
\hline Test 10 & Eation & Horos & CHBCAHO & RAtC & OnIAC \\
\hline $460-1$ & 4.74 & 0.26 & 1.57 & 1 & $7 . \pi$ \\
\hline $460-2$ & 4.74 & 0.26 & 1.54 & & 7.42 \\
\hline $460-3$ & 4.40 & 0.24 & 1.34 & $\vdots$ & 7.07 \\
\hline $460-4$ & 4.78 & 0.22 & 1.37 & $\vdots$ & 7.02 \\
\hline $460-5$ & 4.54 & 0.24 & 1.48 & . & 6.85 \\
\hline \multirow[t]{2}{*}{$460-6$} & 4.33 & 0.26 & 1.57 & $i$ & 6.86 \\
\hline & $!$ & & & $i$ & 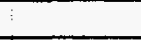 \\
\hline Average & 4.60 & 0.25 & 148 & $\vdots$ & 7.16 \\
\hline Std. Dev. & 0.18 & 0.01 & 0.10 & & 0.36 \\
\hline CVK & 3.88 & 5.99 & 6.90 & 4 & 5.04 \\
\hline
\end{tabular}




\section{Part IV}

\section{Emissions Comparison of Ethanol vs. Diesel}

Six-page figures are provided in this package. The figures in 5 page are the components of emissions in 3 different driving cycles and in the 2-year tests. One page contains the tested fuel economy for the 3 trucks. The average values were plotted in the figures. 

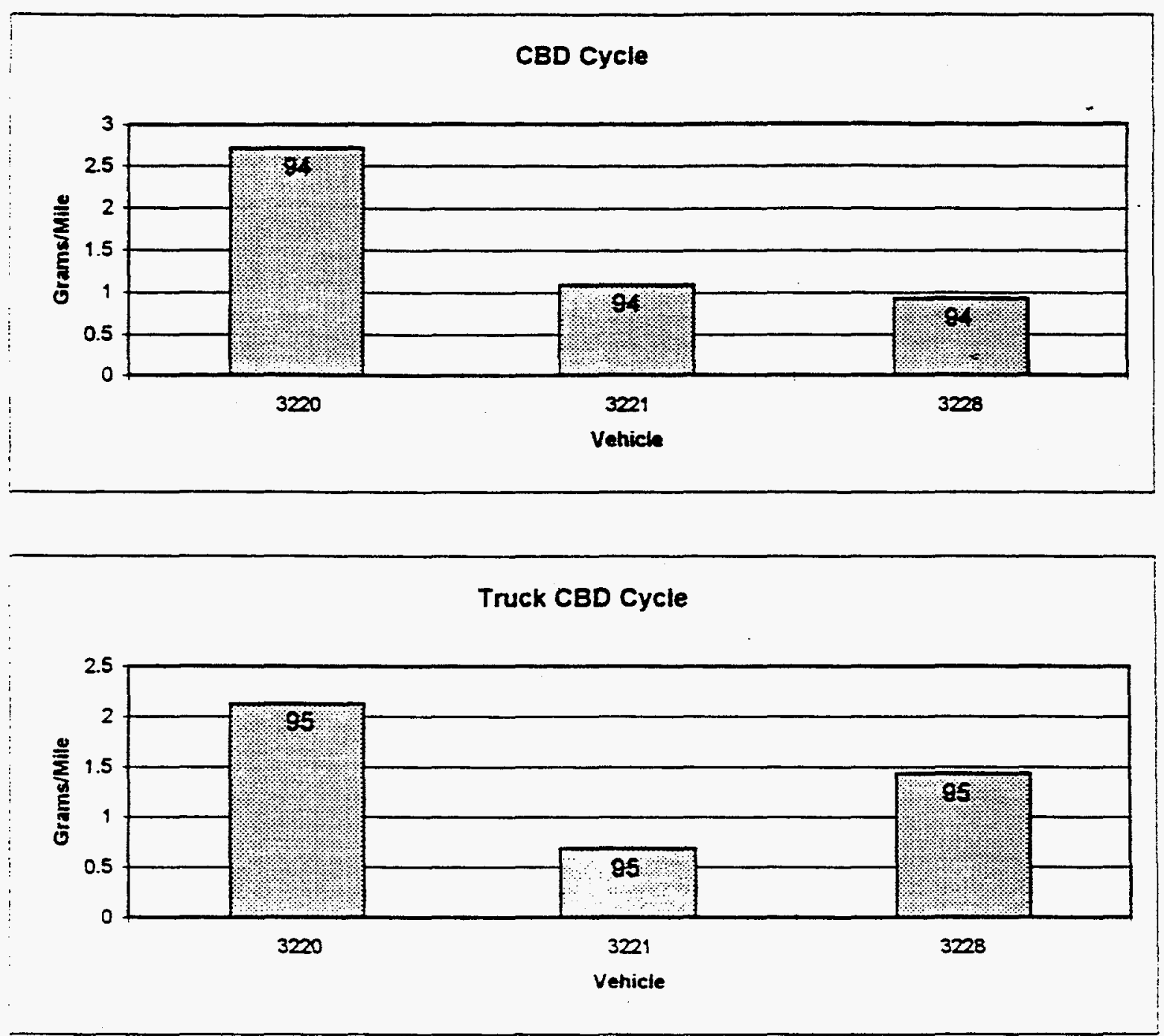

Wvu 5 Peak Truck Cycle

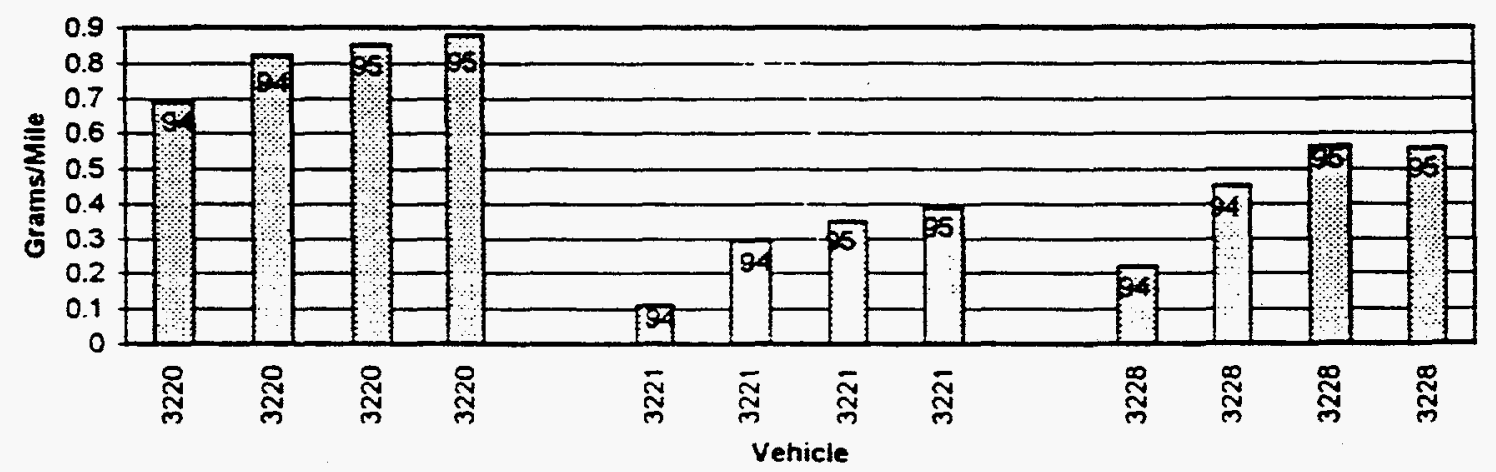



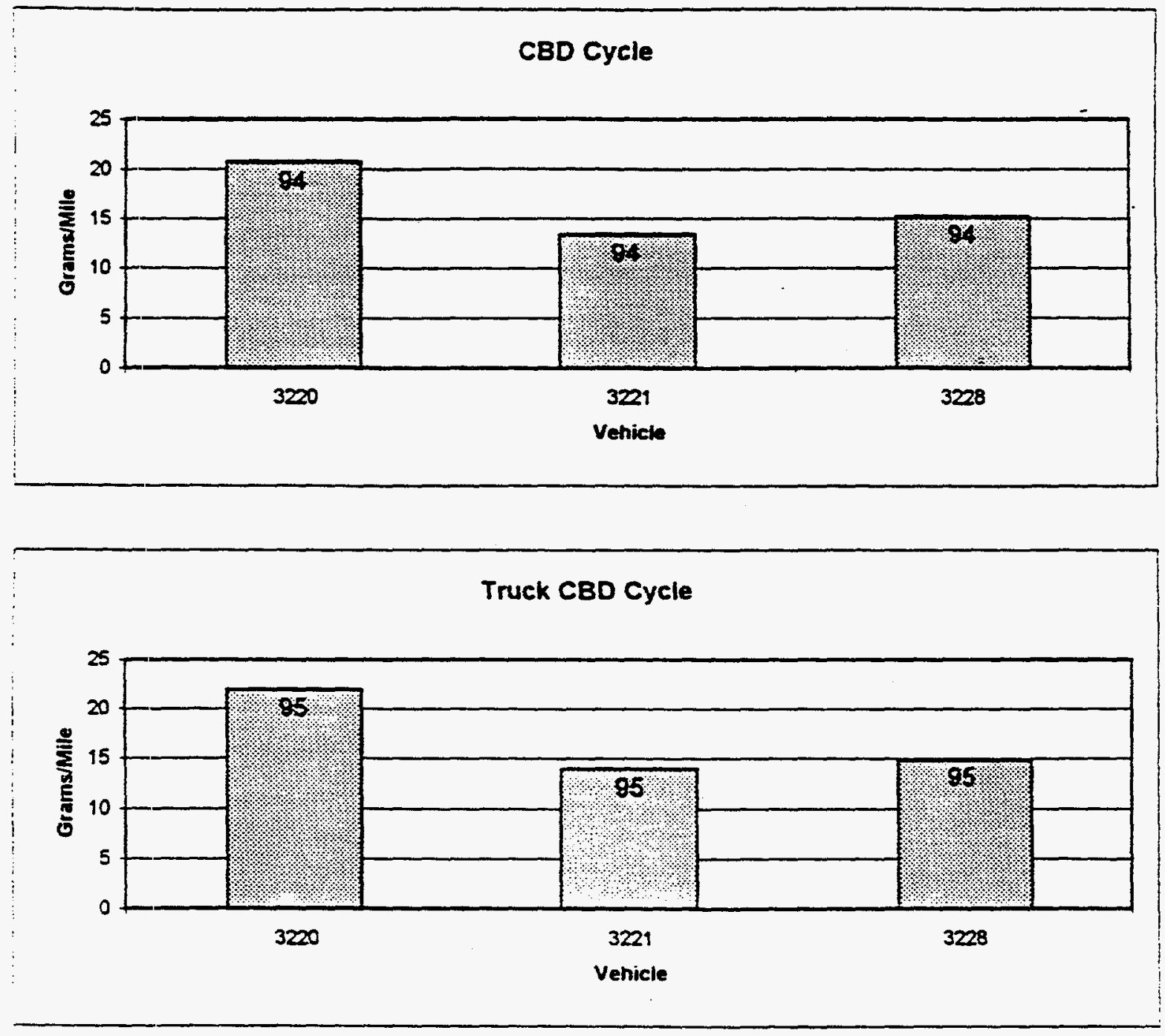

WvU 5 Peak Truck Cycle

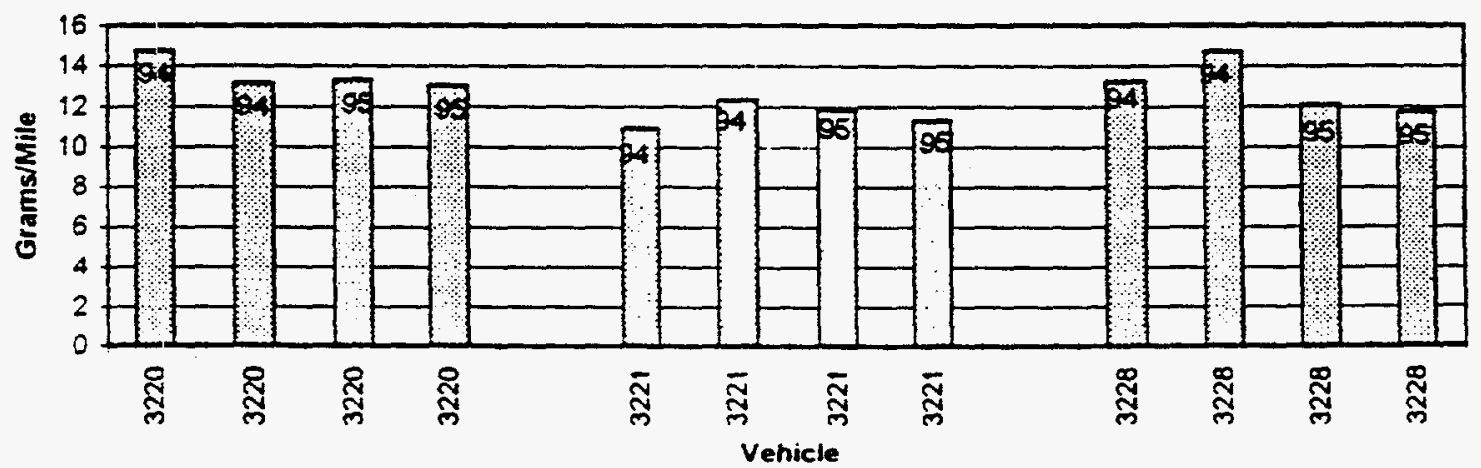



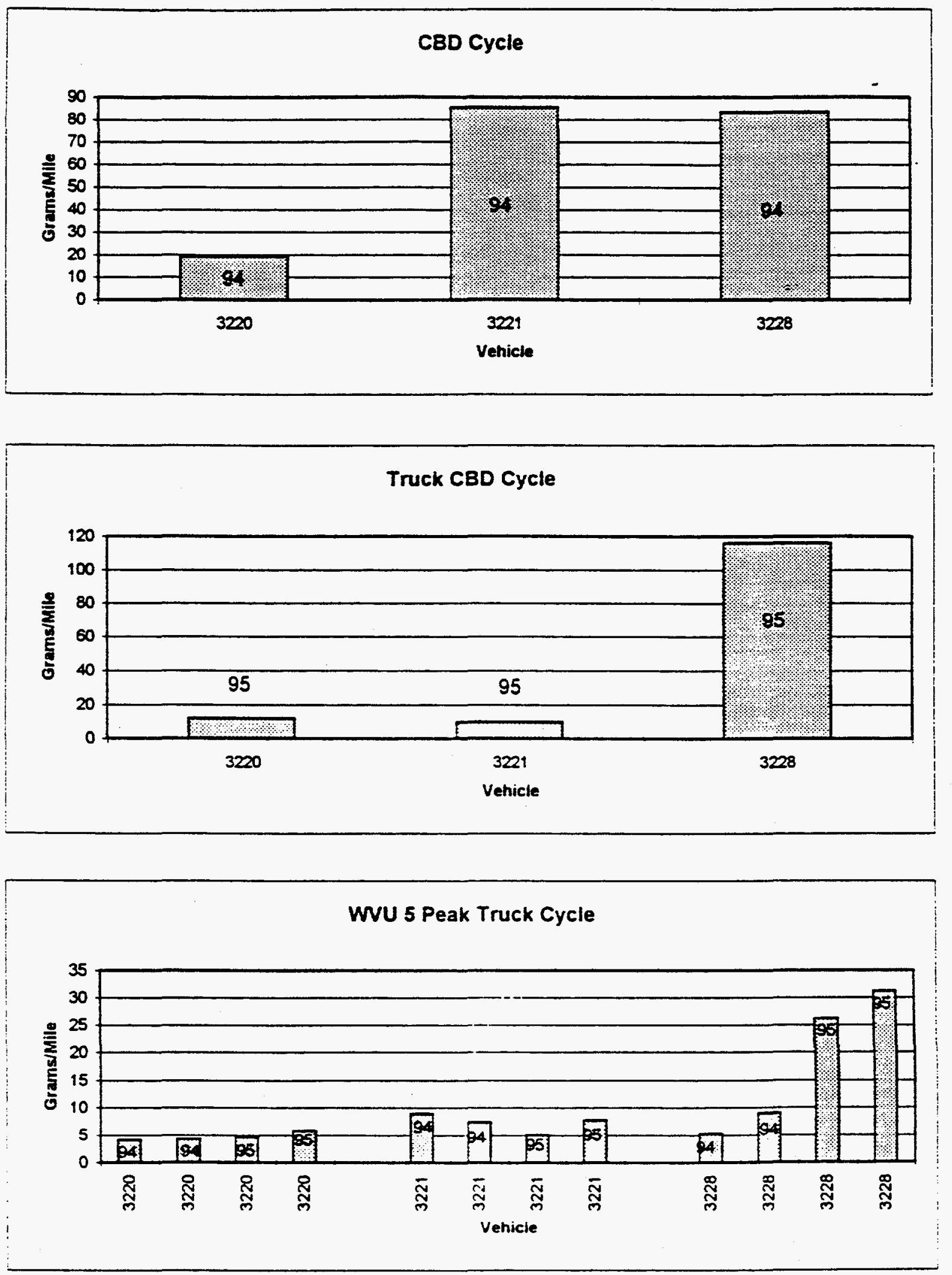

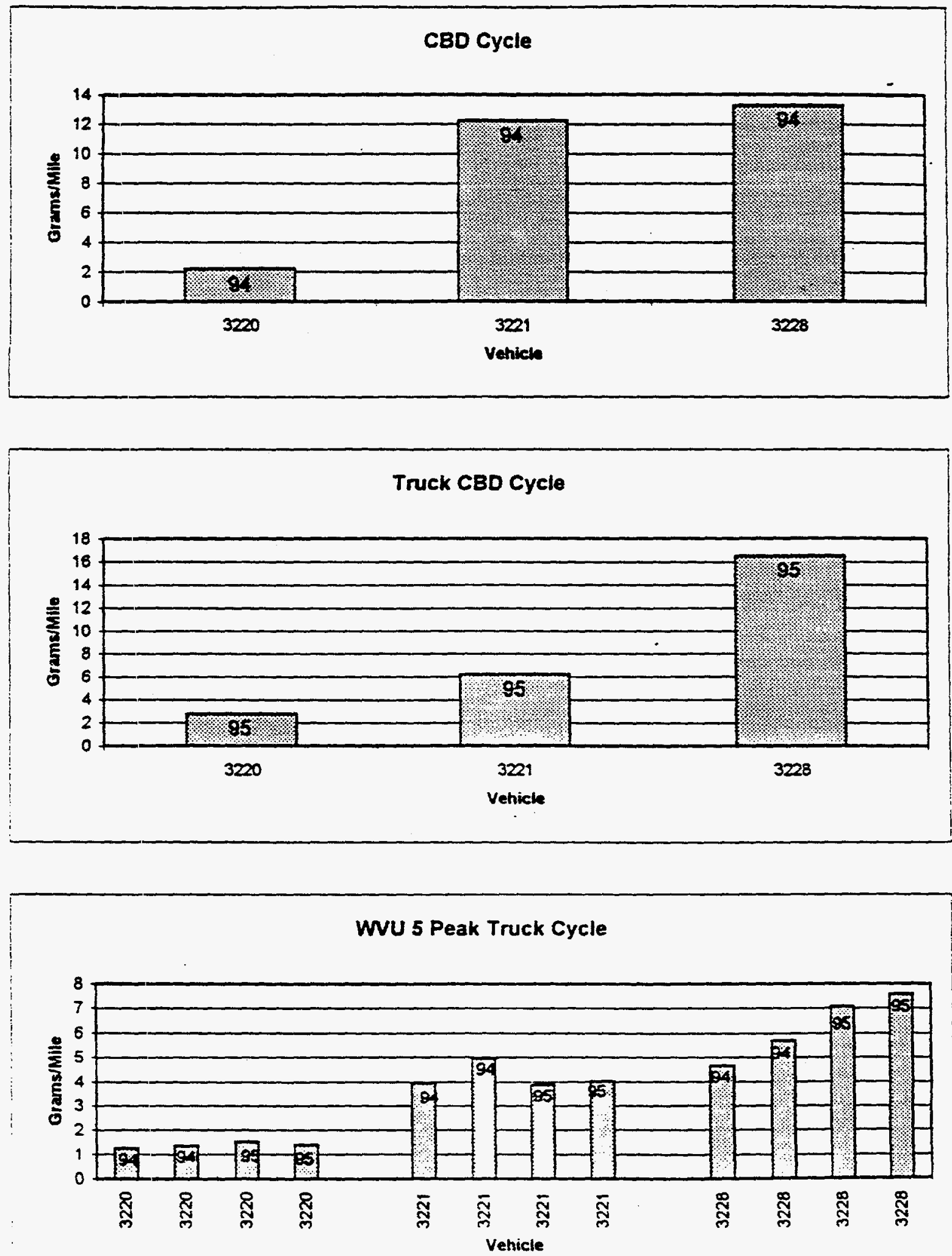

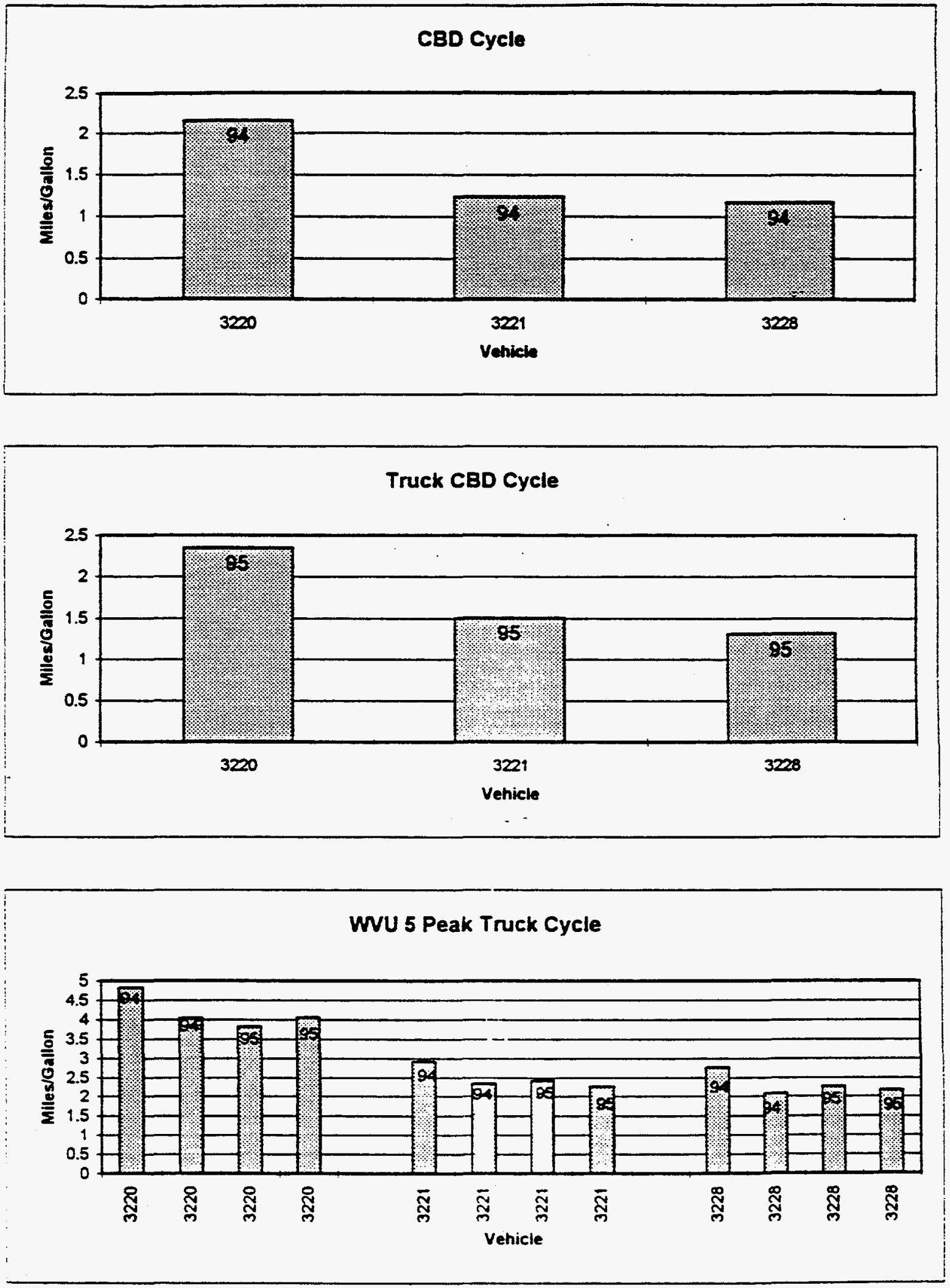

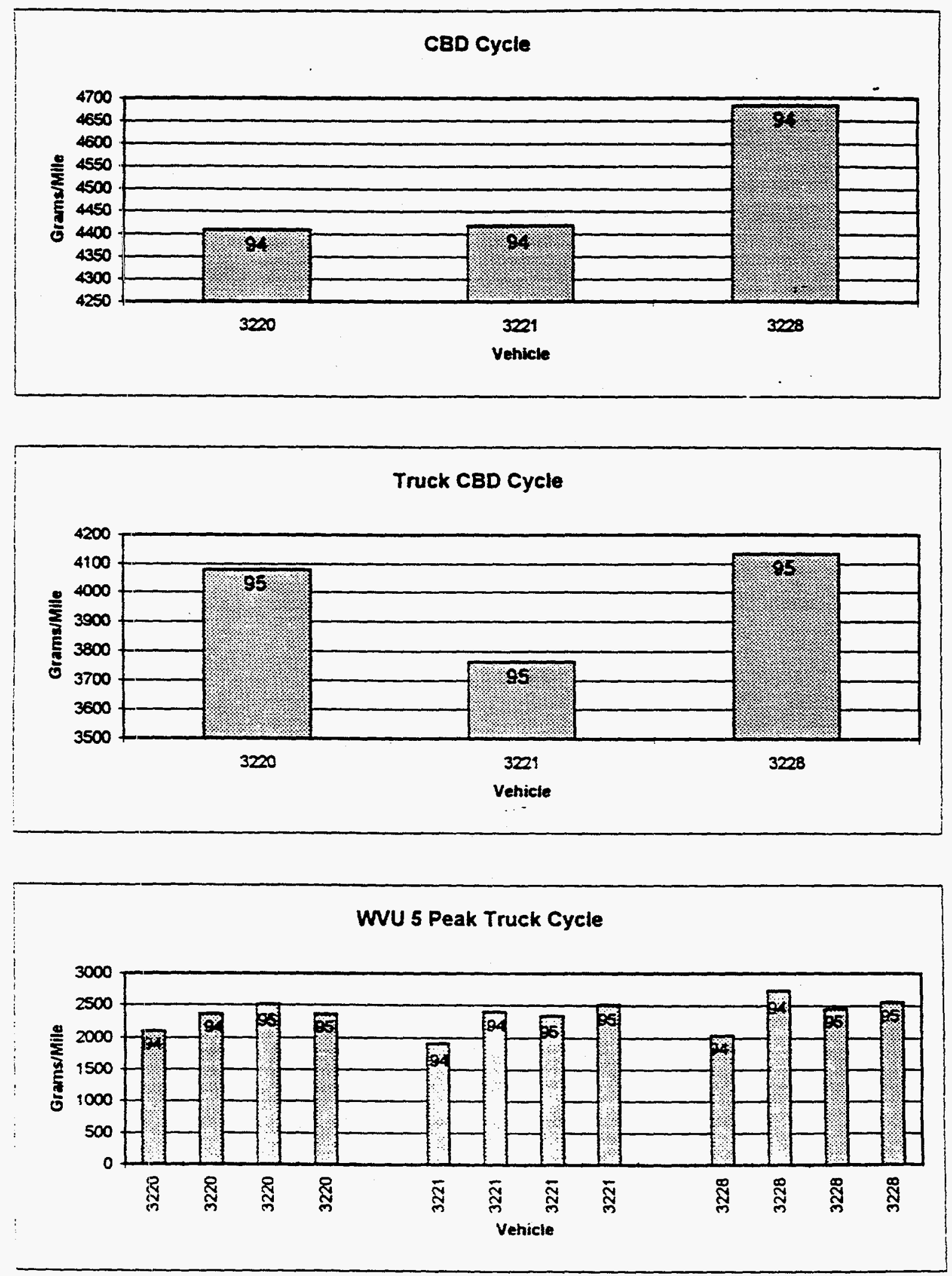


\section{REPORT DOCUMENTATION PAGE}

Form Approved

OMB NO. 0704-0188

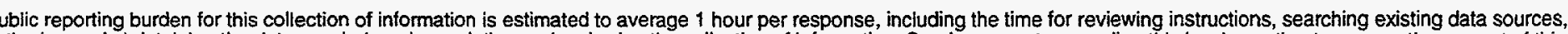

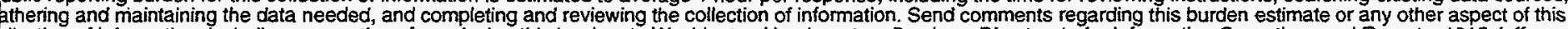

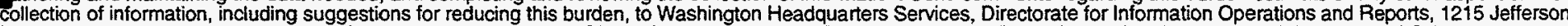

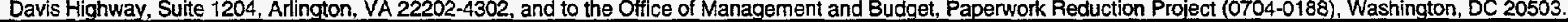

\begin{tabular}{|l|l|l|}
\hline AGENCY USE ONLY (Leave blank) & $\begin{array}{l}\text { 2. REPORT DATE } \\
\text { January 1998 }\end{array}$ & $\begin{array}{l}\text { 3. REPORT TYPE AND DATES COVERED } \\
\text { Subcontract report }\end{array}$ \\
\hline
\end{tabular}

\begin{tabular}{l} 
4. TITLE AND SUBTITLE \\
ennepin County's Experience with Heavy-Duty Ethanol Vehicles \\
\hline AUTHOR(S) \\
ucking Research institute and Hennepin County Central
\end{tabular}

7. PERFORMING ORGANIZATION NAME(S) AND ADDRESS(ES)

rucking Research Institute

5. FUNDING NUMBERS

(C) ZAZ-3-12223-01-107183

(TA) FU803330

$200 \mathrm{Mill}$ Road

Alexandria, VA 22314-4677

ennepin County Central

Do Washington Avenue

Hopkins, MN 55343

SPONSORING/MONITORING AGENCY NAME(S) AND ADDRESS(ES)

wational Renewable Energy Laboratory

1617 Cole Boulevard

Folden, CO 80401-3393

8. PERFORMING ORGANIZATION

REPORT NUMBER

1. SUPPLEMENTARY NOTES

\section{2a. DISTRIBUTION/AVAILABILITY STATEMENT}

National Technical information Service

U.S. Department of Commerce

285 Port Royal Road

pringtield, VA 22161 12b. DISTRIBUTION CODE

UC-1504

13. ABSTRACT (Maximum 200 words) Hennepin County, Minnesota, field-tested two heavy-duty snowplow/road maintenance trucks fueled by ethanol. The objective of is program was to collect data from original equipment manufacturer alternative fuel heavy-duty trucks, along with comparable data from a similarly configured dieselpwered vehicle, to establish economic, emissions, performance, and durability data for the alternative fuel technology. A considerable amount of data was collected, and project participants called the project a success.

\begin{tabular}{|c|c|c|c|}
\hline \multirow{2}{*}{\multicolumn{3}{|c|}{$\begin{array}{l}\text { 14. SUBJECT TERMS } \\
\text { ternative fuels, ethanol, heavy-duty vehicles }\end{array}$}} & \multirow{2}{*}{$\begin{array}{l}\text { 15. NUMBER OF PAGES } \\
86 \\
\text { 16. PRICE CODE }\end{array}$} \\
\hline & & & \\
\hline $\begin{array}{l}\text { SECURITY CLASSIFICATION } \\
\text { OF REPORT }\end{array}$ & $\begin{array}{l}\text { 18. SECURITY CLASSIFICATION } \\
\text { OF THIS PAGE }\end{array}$ & $\begin{array}{l}\text { 19. SECURITY CLASSIFICATION } \\
\text { OF ABSTRACT }\end{array}$ & 20. LIMITATION OF ABSTRACT \\
\hline
\end{tabular}

